\title{
Beam Spin Asymmetry Measurements for Two Pion Photoproduction at CLAS
}

\author{
Mark David Anderson
}

Submitted in fulfilment of the requirements for the degree of Doctor of Philosophy

Nuclear Physics Group

School of Physics and Astronomy

College of Science and Engineering

University of Glasgow

September 2015 


\section{Abstract}

The overarching goal of this analysis, and many like it, is to develop our understanding of the strong force interactions within the nucleon by examining the nature of their excitation spectra. As the resonances of these spectra have very short lifetimes $\left(\tau=1 \times 10^{-23} \mathrm{~s}\right)$ and often have very similar masses, it is often impossible to directly observe resonances in the excitation spectra of nucleons. Polarization observables allow us to study the resonances by looking at how they affect the spin state of final state particles. The beam asymmetry is a polarization observable that allows us to detect the sensitivity of these resonances, and other transition mechanisms, to the electric vector orientation of incident photons.

Presented in this thesis are first measurements of the beam asymmetries in the resonant region for the reaction channel $\gamma p \rightarrow p \pi^{+} \pi^{-}$focusing on the intermediate mesonic states $\rho^{0}$ and $f^{0}$, and the final state pions. The analysis used data from the $\mathrm{g} 8 \mathrm{~b}$ experiment undertaken at the Thomas Jeffereson National Accelerator Facility(JLab), the first experiment at JLab to use a linearly polarized photon beam. Using the coherent Bremsstrahlung facility and the CLAS detector of Hall B at JLab allowed for many multi-channel reactions to be detected and the first measurements of many polarization observables including those presented here.

A brief overview of the theoretical framework used to undertake this analysis is given, followed by a description of the experimental details of the facilities used, then a description of the calibration of the Bremsstrahlung tagging facility which the author undertook, and finally the analysis is presented and the resulting measurements. 


\section{Declaration}

I declare that I composed this thesis in its entirety and that the work presented was completed by myself, except in the cases where the works of others have been explicitly referenced.

Mark David Anderson 


\section{Contents}

Abstract i

1 Introduction 1

1.1 The Nucleon and other Hadrons _. . . . . . . . . . . . . . . . 1

1.2 Quantum Chromodynamics . . . . . . . . . . . . . . 3

1.3 Hadron Spectroscopy . . . . . . . . . . . . . . . . . . 4

1.4 Quark Models . . . . . . . . . . . . . . . . . . . 5 5

1.5 Photoproduced Proton Spectra . . . . . . . . . . . . . 7

2 Two Pion Photoproduction 9

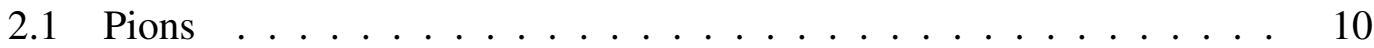

2.2 The $\rho^{0}$ Meson . . . . . . . . . . . . . . . . 10

2.3 Vector Meson Photoproduction Kinematics _ . . . . . . . . . . . . 12

2.4 Helicity Amplitudes and Polarization Observables . . . . . . . . . . . 15

2.5 Deriving Angular Distibrution $\ldots \ldots \ldots . \ldots . \ldots$

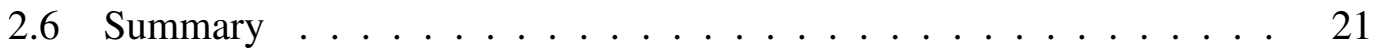

3 Previous Measurements $\quad 23$

$3.1 \quad \rho^{0}$ Photoproduction Results . . . . . . . . . . . . . . . . . 23

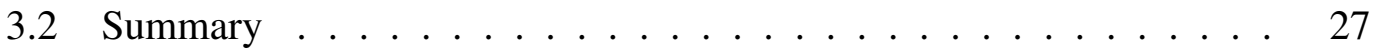

4 Experimental Details $\quad 29$

4.1 Continuous Electron Beam Accelerator Facility . . . . . . . . . . . . 29 
4.2 Electron Beamline . . . . . . . . . . . . . . 31

4.3 The Tagging Spectrometer . . . . . . . . . . . . 32

4.4 Coherent Bremsstrahlung . . . . . . . . . . . . . . . . . . . 34

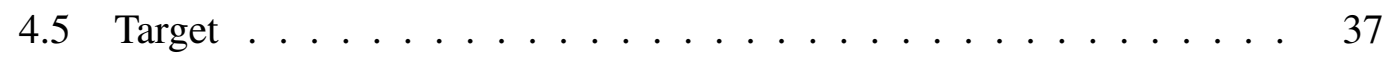

4.6 The CEBAF Large Acceptance Detector . . . . . . . . . . . . . . . 37

4.7 Trigger System . . . . . . . . . . . . . . . . . . . 4 43

4.8 Data Acquisition System . . . . . . . . . . . . . . 43

4.9 Summary . . . . . . . . . . . . . . . . . . 44

5 Photon Polarization $\quad 45$

5.1 Theory .............................. 45

5.2 Enhancement Spectra . . . . . . . . . . . . . . . . . . . 48 48

5.3 Moving Diamonds $\ldots \ldots \ldots \ldots$

5.4 Deriving a Fit $\ldots \ldots \ldots \ldots \ldots \ldots$

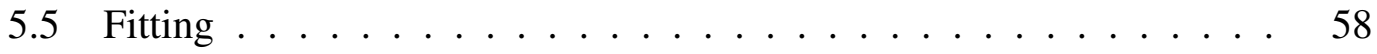

5.6 Polarization Tables . . . . . . . . . . . . . . . 66

$\begin{array}{llr}6 & \text { Data Analysis } & 68\end{array}$

6.1 Particle Identification . . . . . . . . . . . . . . . . . . 68

6.2 Channel Selection . . . . . . . . . . . . . . . . . . . 97

6.3 Fitting $\mathrm{M}\left(\pi^{+} \pi^{-}\right)$Distributions . . . . . . . . . . . . 108

6.4 Beam Asymmetry Extraction . . . . . . . . . . . . . . . . 111

6.5 The Need for a 2-D Fit . . . . . . . . . . . . . . . . 114

6.6 Summary . . . . . . . . . . . . . . . . . . . 121

$\begin{array}{llr}7 & \text { Results } & 122\end{array}$

8 Conclusion and Discussion 138

8.1 Summary . . . . . . . . . . . . . . . . . 139

$\begin{array}{lll}\text { A Invariant Mass Plots and Fits } & 141\end{array}$ 
B Asymmetry Fits 


\section{List of Figures}

1.1 Baryon Multiplets. . . . . . . . . . . . . . . 2

1.2 QCD coupling constant against energy. . . . . . . . . . . . . . . 4

1.3 Table of measured and predicted resonances. . . . . . . . . . . . 6

1.4 Total cross section of $\gamma p \rightarrow N^{*} \ldots \ldots \ldots \ldots$

2.1 Diagram of the kinematics involved in a scattering reaction as viewed in the centre-of-mass frame. . . . . . . . . . . . . . . . 12

2.2 Born diagrams of the different processees which contribute to the photoproduction of the $\rho^{0} \ldots \ldots \ldots \ldots \ldots \ldots$

2.3 A diagram showing the different planes and angles involved in the photoproduction of $\rho^{0}$ mesons. . . . . . . . . . . . . . . 15

3.1 Differential cross section results from the ABBHHM collaboration. . 24

3.2 Beam asymmetry measurements for $\rho^{0}$ above the resonant energy region. 25

3.3 Beam asymmetry measurements for $\omega$ mesons as a function of $\theta$. . . 26

3.4 Cross sections measurements for $\rho^{0}$ at CLAS as function of $-t$. . . . 27

3.5 Cross sections measured for $f^{0}$ a function of $-t \ldots \ldots 28$

4.1 A schematic of CEBAF showing the injector, LINACs, and recircula-

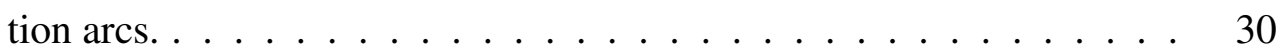

4.2 An example harp scan showing an ideal beam profile in both the $\mathrm{x}$ and

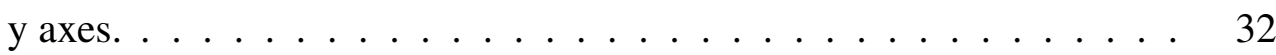

4.3 Diagram of the tagger spectrometer. . . . . . . . . . . . 34 
4.4 A schematic of the tagger facility and all of the associated systems. . . 35

4.5 Typical photon spectrum using different radiator. . . . . . . . . . 36

4.6 3D diagram of the CLAS detector and its sub-systems. . . . . . . . 37

4.7 Further diagrams of the CLAS detector. . . . . . . . . . . 38

4.8 3D diagram of the Start Counter. . . . . . . . . . . . . . . . . 40

4.9 Diagram showing the layers and cells of the CLAS Drift Chambers. . 41

4.10 Diagram of one section of the Time-of-Flight system. . . . . . . . . . 42

5.1 A typical enhancment plot for the measured coherent edge position of $1.109 \mathrm{GeV}$ for the $1.1 \mathrm{GeV}$ datase. . . . . . . . . . . . . . 47

5.2 The angles involved for coherent bremsstrahlung from a diamond in the 100 orientation. . . . . . . . . . . . . . . . . . 48

5.3 A typical timing plot using the calibrated tagger information. . . . . . 49

5.4 The the amorphous energy spectrum for the $1.1 \mathrm{GeV}$ data for the prompt region. . . . . . . . . . . . . . . . . 50

5.5 The amorphous energy spectrum for the $1.1 \mathrm{GeV}$ data for the random region. .......................... 50

5.6 The corrected amorphous energy spectrum for the $1.1 \mathrm{GeV}$ data. . . . 51

5.7 The the parallel polarization energy spectrum for the $1.1 \mathrm{GeV}$ data for

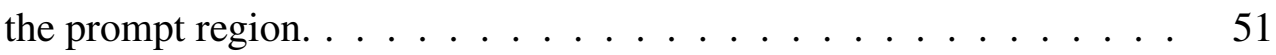

5.8 The parallel polarization energy spectrum for the $1.1 \mathrm{GeV}$ data for the random region. . . . . . . . . . . . . . . 52

5.9 The corrected parallel polarization energy spectrum for the $1.1 \mathrm{GeV}$ data. 52

5.10 The shift for the coherent edge position for each beam setting for the g8b data. . . . . . . . . . . . . . . . . . 53

5.11 The corrected parallel polarization energy spectrum as a function of the measured coherent edge position for the $1.1 \mathrm{GeV}$ data. . . . . . . 58

5.12 The differenet measured coherent edge values for the $1.1 \mathrm{GeV}$ coherent edge dataset for the parallel polarization data. 
5.13 First pass of fitting of an enhancment plot showing the fit for a specific measured coherent edge and the corresponding polarization. . . . . . 61

5.14 Fit parameters from the first application of the enhancment fit. . . . . 62

5.15 Second pass of fitting of an enhancment plot showing the fit for a specific measured coherent edge and the corresponding polarization. . . .

5.16 The polarization values as a function of coherent edge position for a specific e-counter corresponding to $E_{\gamma}=1103 \mathrm{MeV}$. . . . . . . . .

6.1 The measured beta of the detected particles as a function of the momentum. . . . . . . . . . . . . . .

6.2 The difference between the measured beta of the detected particles and the calculated value as a function of the momentum. . . . . . . . .

6.3 The difference between the measured beta of the detected particles and the calculated value as a function of the momentum after cuts. . . . .

6.4 The measured beta of the detected particles as a function of the momentum after cuts. . . . . . . . . . . . . . . 74

6.5 The z-vertex distributions for the detected particles. . . . . . . . 76

6.6 The angular distribution for the detected particles. . . . . . . . . . 77

6.7 The angular distribution for the detected particles after fiducial cuts. . 78

6.8 The angular distribution for the reconstructed missing particles. . . . . 79

6.9 The multiplicity of photons per event. . . . . . . . . . 80

6.10 Timing difference between the hadronic TOF timing and the associated photon's timing from the tagging system. . . . . . . . 81

6.11 Timing difference between the hadronic TOF timing and the associated photon's timing from the tagging system after the best photon procedure. 82

6.12 Timing difference between the positive pion TOF timing and the associated photon's timing from the tagging system for the events with a

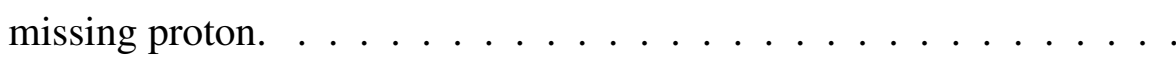


6.13 Timing difference between the positive pion TOF timing and the associated photon's timing from the tagging system after the best photon procedure for the events with a missing proton. . . . . . . . . .

6.14 The momentum of the final state particles as a function of the difference between their timing vertex and the corresponding incident pho-

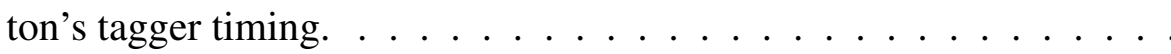

6.15 The momentum of the final state particles as a function of the difference between their timing vertex and the corresponding incident photon's tagger timing with after cuts. . . . . . . . .

6.16 The energy loss calculated using the ELOSS package for the detected

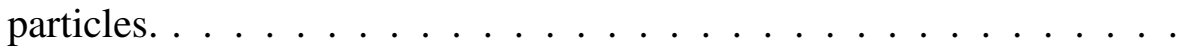

6.17 The energy loss calculated using the ELOSS package as a function of momentum for the detected particles. . . . . . . . . . . . .

6.18 The angle between the proton and the reconstructed particle from which the pions decayed. . . . . . . . . . . . . . . . . . . 89

6.19 The missing mass squared of the exclusive topology. . . . . . . . . . 90

6.20 The missing mass squared distributions for the case of one undetected pion.

6.21 The missing mass squared distributions for the case of the undetected

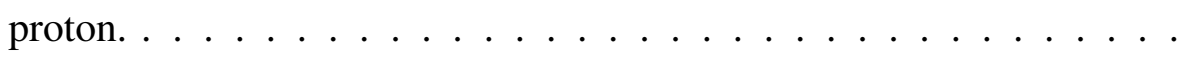

6.22 The measured beta of the reconstructed particles as a function of the

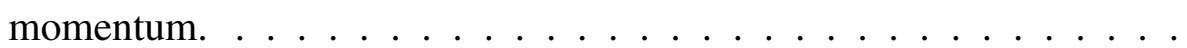

6.23 The difference between the measured beta of the reconstructed particles and the calculated value as a function of the momentum. . . . . .

6.24 The difference between the measured beta of the reconstructed particles and the calculated value as a function of the momentum after cuts.

6.25 The measured beta of the reconstructed particles as a function of the momentum after cuts. . . . . . . . . . . . 
6.26 Invariant mass of the two pions in different kinematic regions. . . . .

6.27 Invariant mass of the other two contribution channels for the whole dataset. . . . . . . . . . . . . . . . . 98

6.28 Invariant mass of the other two contribution channels for the 2100 MeV dataset only. . . . . . . . . . . . . . . . . . . . . . 98

6.29 The signal background separation for $\omega$ mesons using data from CLAS. 99

6.30 Example of the asymmetry for the $\Delta^{++}$as a function of the invariant mass of the two pions. . . . . . . . . . . . . . 101

6.31 The coarse cut on the $\Delta$ resonances. . . . . . . . . . . . . . 102

6.32 The Dalitz plots of the invariant mass of proton and the $\pi^{+}$against that of the two pions for different $W$ regions. . . . . . . . . . . . . 103

6.33 The Dalitz plots of the invariant mass of proton and the $\pi^{-}$against that of the two pions for different $W$ regions. . . . . . . . . . . . . . 104

6.34 The effect of cutting the regions associated with the $\Delta$ resonances. . . 105

6.35 The $\cos \theta_{C M}$ distribution for the dataset spanning centre of mass region $1.85-2.23 \mathrm{GeV}$

6.36 The $\mathrm{W}$ distribution for the dataset spanning the entire $\cos \theta_{C M}$ distribution.

6.37 Invariant mass of the two pions in different kinematic regions fitted with the function shown in equation $6.6 \ldots \ldots \ldots$

6.38 The region for different kinematic bins used for extracting the beam asymmetry in the region of the $\rho^{0}$ and $f^{0} \ldots \ldots \ldots \ldots$

6.39 A diagram showing the different planes and angles involved in the photoproduction of $\rho^{0}$ mesons. . . . . . . . . . . . . . . . . . . 112

6.40 An example of the fit described in equation 6.13 applied to an angular distribution asymmetry. . . . . . . . . . . . . . . . . . . 114

6.41 $\Phi$ as a function of $\cos \theta_{h}$ in different $\phi_{h}$ regions to show the lack of sensitivity of $\Phi$ with respect to $\cos \theta_{h} \ldots \ldots \ldots \ldots$ 
6.42 $\Phi$ as a function of $\phi_{h}$ in different $\cos \theta_{h}$ to show the lack of sensitivity of $\Phi$ with respect to $\cos \theta_{h} \ldots \ldots \ldots \ldots$. . . . . . . . . . 116

6.43 An example of the fit described in equation 6.14 applied to a 2-D angular distribution asymmetry of $\Phi$ against $\phi_{h} \ldots \ldots$. . . . . . . . 117

$6.44 \Phi$ as a function of $\phi_{h}$ integrated over the full range of $\cos \theta_{h}$ using semi-inclusive channels and the exlcusive channel. . . . . . . . . 118

6.45 An example of the fit described in equation 6.13 applied to an one dimension angular distribution asymmetry of $\phi_{f^{0}} \ldots \ldots \ldots$

6.46 An example of the fit described in equation 6.13 applied to an one dimension angular distribution asymmetry of $\phi_{\pi^{+}} \ldots \ldots \ldots \ldots$

6.47 An example of the fit described in equation 6.13 applied to an one dimension angular distribution asymmetry of $\phi_{\pi^{+}} \ldots \ldots \ldots \ldots$

7.1 Beam asymmetry extracted values for $\rho^{0}$ as a function of $\cos \theta_{C M}$ for different $W$ regions. . . . . . . . . . . . . . . . . . . . . . . 123

7.2 Cross sections measured for $\rho^{0}$ a function of $-t$ for different $\mathrm{W}$ regions spanning 1.87-2.12 GeV.

7.3 Beam asymmetry extracted values for $f^{0}$ as a function of $\cos \theta_{C M}$ for different $W$ regions. . . . . . . . . . . . . . . . . . . . . . . . . . . . 124

7.4 Cross sections extracted measured for $f^{0}$ a function of $-t$ for photon energy range $E_{\gamma}=3.0-3.8 \mathrm{GeV}$.

7.5 Beam asymmetry extracted values for the $\pi^{+}$as a function of $M\left(\pi^{+} \pi^{-}\right)$ for different $\mathrm{W}$ region 1 .

7.6 Beam asymmetry extracted values for the $\pi^{+}$as a function of $M\left(\pi^{+} \pi^{-}\right)$ for $\mathrm{W}$ region $2 . \ldots \ldots \ldots$. . . . . . . . . . . . . . . 127

7.7 Beam asymmetry extracted values for the $\pi^{+}$as a function of $M\left(\pi^{+} \pi^{-}\right)$ for $\mathrm{W}$ region $3 . \ldots \ldots \ldots \ldots$

7.8 Beam asymmetry extracted values for the $\pi^{+}$as a function of $M\left(\pi^{+} \pi^{-}\right)$ for $\mathrm{W}$ region 4. 
7.9 Beam asymmetry extracted values for the $\pi^{+}$as a function of $M\left(\pi^{+} \pi^{-}\right)$ for $\mathrm{W}$ region $5 . \ldots \ldots \ldots$

7.10 Beam asymmetry extracted values for the $\pi^{+}$as a function of $M\left(\pi^{+} \pi^{-}\right)$ for $\mathrm{W}$ region 6.

7.11 Beam asymmetry extracted values for the $\pi^{-}$as a function of $M\left(\pi^{+} \pi^{-}\right)$ for $\mathrm{W}$ region $1 . \ldots \ldots \ldots \ldots$

7.12 Beam asymmetry extracted values for the $\pi^{-}$as a function of $M\left(\pi^{+} \pi^{-}\right)$ for $\mathrm{W}$ region $2 \ldots \ldots \ldots \ldots$

7.13 Beam asymmetry extracted values for the $\pi^{-}$as a function of $M\left(\pi^{+} \pi^{-}\right)$ for $\mathrm{W}$ region 3

7.14 Beam asymmetry extracted values for the $\pi^{-}$as a function of $M\left(\pi^{+} \pi^{-}\right)$ for $\mathrm{W}$ region 4.

7.15 Beam asymmetry extracted values for the $\pi^{-}$as a function of $M\left(\pi^{+} \pi^{-}\right)$ for $\mathrm{W}$ region $5 . \ldots \ldots \ldots \ldots$

7.16 Beam asymmetry extracted values for the $\pi^{-}$as a function of $M\left(\pi^{+} \pi^{-}\right)$ for $\mathrm{W}$ region 6.

A.1 The fit described in section 6.3 for each kinematic region for the forward polar angle region.

A.2 The fit described in section 6.3 for each kinematic region for the backward polar angle region.

A.3 The fit described in section 6.3 for each kinematic region for the forward polar angle region. Scaled.

A.4 The fit described in section 6.3 for each kinematic region for the backward polar angle region. Scaled.

B.1 The fit described in section 6.4 for each kinematic region for the forward polar angle region for extracting $\Sigma_{\rho^{0}} \ldots \ldots \ldots \ldots$. . . . . 147 
B.2 The fit described in section 6.4 for each kinematic region for the backward polar angle region for extracting $\Sigma_{\rho^{0}} \ldots \ldots \ldots \ldots$

B.3 The fit described in section 6.5.1 for each kinematic region for extracting $\Sigma_{f^{0}} \ldots \ldots \ldots \ldots \ldots \ldots \ldots \ldots \ldots \ldots$

B.4 Asymmetry fits for the $\pi^{-}$in each $\cos \theta$ bin in the forward region in the second $\mathrm{W}$ region and in lower region of $\mathrm{M}\left(\pi^{+} \pi^{-}\right) \ldots \ldots \ldots$

B.5 Asymmetry fits for the $\pi^{-}$in each $\cos \theta$ bin in the backward region in the second $\mathrm{W}$ region and in lower region of $\mathrm{M}\left(\pi^{+} \pi^{-}\right) \ldots \ldots \ldots 1$

B.6 Asymmetry fits for the $\pi^{-}$in each $\cos \theta$ bin in the forward region in the second $\mathrm{W}$ region and in upper region of $\mathrm{M}\left(\pi^{+} \pi^{-}\right) \ldots \ldots \ldots$

B.7 Asymmetry fits for the $\pi^{-}$in each $\cos \theta$ bin in the backward region in the second $\mathrm{W}$ region and in upper region of $\mathrm{M}\left(\pi^{+} \pi^{-}\right) . \ldots . . .153$

B.8 Asymmetry fits for the $\pi^{+}$in each $\cos \theta$ bin in the forward region in the second $\mathrm{W}$ region and in lower region of $\mathrm{M}\left(\pi^{+} \pi^{-}\right) . \quad \ldots \ldots . .154$

B.9 Asymmetry fits for the $\pi^{+}$in each $\cos \theta$ bin in the backward region in the second $\mathrm{W}$ region and in lower region of $\mathrm{M}\left(\pi^{+} \pi^{-}\right) . \quad \ldots \ldots 155$

B.10 Asymmetry fits for the $\pi^{+}$in each $\cos \theta$ bin in the forward region in the second $\mathrm{W}$ region and in upper region of $\mathrm{M}\left(\pi^{+} \pi^{-}\right) \ldots \ldots \ldots$

B.11 Asymmetry fits for the $\pi^{+}$in each $\cos \theta$ bin in the backward region in the second $\mathrm{W}$ region and in upper region of $\mathrm{M}\left(\pi^{+} \pi^{-}\right) . \quad \ldots . . .157$ 


\section{List of Tables}

5.1 Part of the polarization table for $\mathrm{g} 8 \mathrm{~b}$ for the dataset with the coherent edge nominally set at $1300 \mathrm{MeV} \ldots \ldots \ldots 6$

5.2 Average polarization values for the different run settings for the $\mathrm{g} 8 \mathrm{~b}$ dataset after the best photon process. . . . . . . . . . . . 67

6.1 The values used for the soft cuts on the mass of the detected particles. $\quad 70$

6.2 The values used for fiducial cuts. . . . . . . . . . . . . . 75

$6.3 \cos \theta_{C M}$ kinematic bins used in the analysis. . . . . . . . . . . 108

$6.4 \mathrm{~W}$ kinematic bins used in the analysis. . . . . . . . . . . . . 108

$6.5 \cos \theta_{C M}$ kinematic bins used in the analysis of the pions. . . . . . 119 


\section{Chapter 1}

\section{Introduction}

Beam asymmetry measurements were made for the reaction channel $\gamma p \rightarrow p \pi^{+} \pi^{-}$. It forms part of a wider effort to characterise the spectra of hadrons in order to aid our understanding of the strong force interactions within the nucleon and other hadrons. In this chapter a brief overview of the historical developments pertinent to our analyis are described. This is followed by a discussion on the theoretical framework used in the analysis. The subsequent chapters deal with previous measurements, experimental details, and the analysis itself.

\subsection{The Nucleon and other Hadrons}

Almost a century ago, the proton was discovered [1]. About a decade later, the neutron was unearthed [2]. It was postulated that due to their similar characterstics that they were in fact the same particle but in a different state: the nucleon. This led to the introduction of isospin, a quantum number associated with the strong force. It accounted for particles which have different charge states but are affected equally by the strong interaction [3][4].

As the number of discovered particles expanded so too did the number of quantum numbers needed to describe the many facets of the strong force. The baryon number, B, was introduced to tie together the many new particles to the proton, describing it as the 
stable base to which the other similar particles would decay to [5]. It also distinguished mesons from baryons, the former having $\mathrm{B}=0$ and the latter having $\mathrm{B}=1$. And then strangeness, S, was introduced to account for the longer lifetime of some of these new particles [6][7].

In order to bring some order to this large collection of particles, the Eight-fold Way was developed. Particles with similar traits were collected together, namely particles with the same spin and parity. They were plotted with varying degrees of hypercharge, $\mathrm{Y}$, for the different rows, and from negative charge to positive charge on each row. For the multiplets shown here, $\mathrm{Y}=\mathrm{B}+\mathrm{S}$. The two different multiplets shown in figure 1.1 are first the baryon octet where $J^{P}=\frac{1}{2}^{+}$(where $\mathrm{J}$ is spin and $\mathrm{P}$ is parity) and second is the baryon decuplet where $J^{P}=\frac{3}{2}^{+}$[8][9].

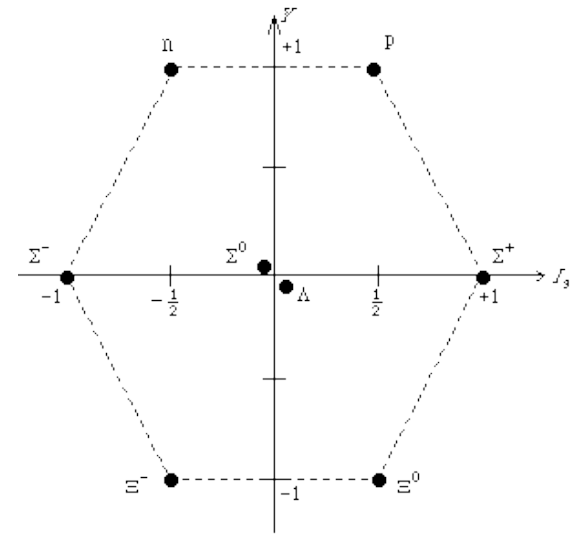

(a) The baryon octet where $J^{P}=\frac{1}{2}^{+}$. Charge increases from left to right, and hypercharge increases from the bottom to the top row.

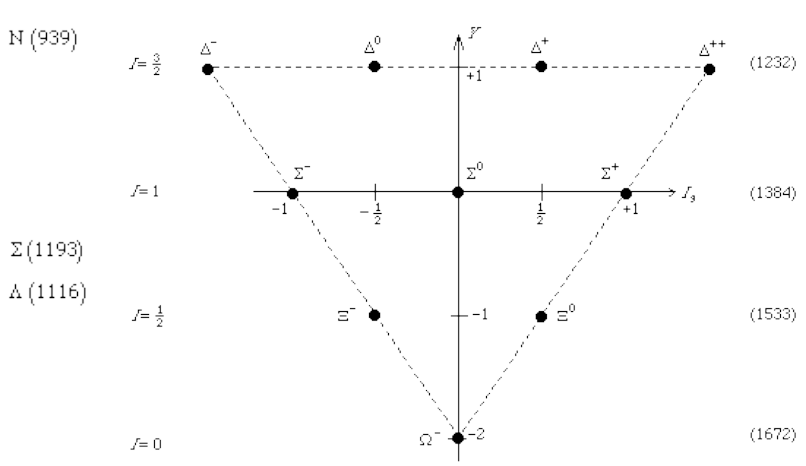

(b) The baryon decuplet where $J^{P}=\frac{3}{2}^{+}$. Charge increases from left to right, and hypercharge increases from the bottom to the top row.

Figure 1.1: Multiplets of similar baryons [10].

This then lead to $\mathrm{SU}(3)$ formalism being used to describe the hadrons and the introduction of quarks and three quark flavours, which are now referred to as up, down, and strange quarks [11][12].

This did not quite cover the whole picture. As quarks are fermions, the $\Delta^{++}$and $\Omega^{-}$baryons would break Pauli's Exclusion Principle; the $\Delta^{++}$baryon possessed three up quarks and all spin aligned, and the $\Omega^{-}$baryon three strange quarks and all spin aligned [13][14]. Yet another SU(3) gauge degree of freedom was proposed; this de- 
gree of freedom was described using colour [15]. The gauge boson binding the different colour charge was called the gluon [16]. And so a simple model of the processes inside the nucleon was deduced by highlighting symmetries between certain particles.

The up and down quarks were then experimentally verified a few years later at the Stanford Linear Accelerator Center (SLAC). This indirectly verified the strange quark also [17][18]. Further quarks were theorised; the charm, bottom, and top quarks [19][20]. In the seventies, the charm and bottom quarks were experimentally confirmed [21][22][23]. However, it was only twenty years ago when the top quark was verified [24].

\subsection{Quantum Chromodynamics}

The actual structure of the nucleon is much more complicated than simply three quarks which give it its quantum numbers and gluons binding them together. The quantum numbers of a hadron do come from the so-called valence quarks, except for its mass and for its spin. In fact, inside the nucleon there are sea quarks, quark-antiquark pairs, and gluonic interactions, which contribute to the mass of the nucleon. The valence quarks only account for $\sim 1 \%$ of the mass of the nucleon, the rest is from the sea quarks and gluonic interactions [25]. The theory widely accepted to describe these strong interactions is Quantum Chromodynamics (QCD). Two peculiar features of this theory are worth mentioning here. First is confinement which states that quarks cannot be observed outside of a hadron and so colour charge must be balanced. The second is the asympotic freedom of the strong force; the further components of the force are separated, the stronger the force gets. Conversely, the higher the energy, the weaker the coupling constant [26]. This is shown in figure 1.2. 


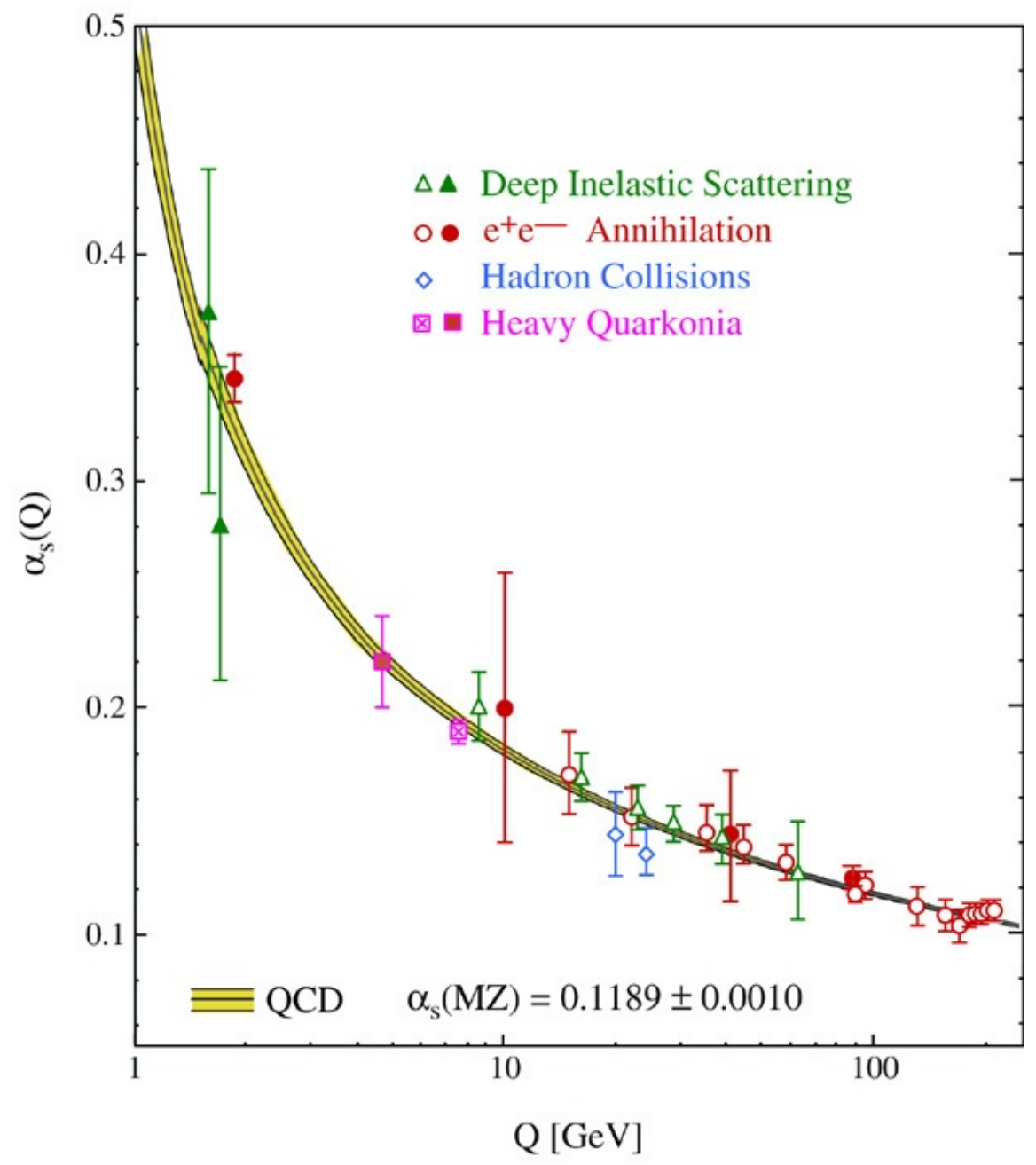

Figure 1.2: The yellow band shows QCD calculations of the coupling constant as a function of energy. Shown alongside this prediction are experimental measurents [27].

\subsection{Hadron Spectroscopy}

It is the aim of this thesis to help garner some information on the nature of the processes within the nucleon. Like any composite system this can been done by studying its excitation spectrum [28]. However, the region where resonances exist happen to be at low energies $\sim 1-2 \mathrm{GeV}$ and hence the coupling constant is high. This means that perturbation calculations cannot be done. Recent advancements in lattice QCD, where a discrete spacetime is formed across a lattice in order to make calculations tractable, have allowed for some theoretical predictions to be calculated [29]. But it is very much in its infancy and so we must still look to other models to help describe this region of 
interest.

\subsection{Quark Models}

The simplest form of the quark model is that which is described above with the majority of the hadron's characteristics being derived from its valance quarks. This is called the constituent quark model. There is presumed to be a symmetric degree of freedom with regards to all three quarks. The spectrum is therefore attained by solving the wave equation for three particles in a potential [30]. A major problem is that these constituent quark models predict many more resonances than have been observed [31].

It is has been suggested that the degrees of freedom should be less than allowing all three valence quarks to be excited. This so-called di-quark model, where two of the quarks form a much stronger bound and therefore are excited much less often, predicts many fewer resonances [32]. But it still predicts some undetected resonances and does not predict some resonances with some experimental evidence suggesting their existence.

Not only are there disparities between which resonances are predicted by these models and the resonances observed experimentally, there are also disparities between predicted masses and measured masses. Predictions from the models described here are shown in figure 1.3. 


\begin{tabular}{|c|c|c|c|c|c|c|}
\hline$J^{P}$ & State & $\begin{array}{l}\text { PDG Mass } \\
\text { Range }\end{array}$ & QM & $\begin{array}{l}\text { Di- } \\
\text { quark }\end{array}$ & RQM & Lattice \\
\hline \multirow{6}{*}{$\frac{1}{2}^{+}$} & $938^{(* * * *)}$ & 938 & 960 & 939 & 939 & $1196 \pm 11$ \\
\hline & $1440^{(* * * *)}$ & $1420-1470$ & 1540 & 1513 & 1518 & $2187 \pm 45$ \\
\hline & $1710^{(* * *)}$ & $1680-1740$ & 1770 & 1768 & 1729 & $2255 \pm 28$ \\
\hline & $1880^{(* *)}$ & $(1835-1915)$ & 1880 & 1893 & 1950 & $2351 \pm 37$ \\
\hline & $2100^{(*)}$ & $(2030-2200)$ & 1975 & - & 1996 & $2244 \pm 28$ \\
\hline & - & - & 2065 & - & 2009 & $2544 \pm 51^{*}$ \\
\hline \multirow{4}{*}{$\frac{3}{2}^{+}$} & $1720^{(* * * *)}$ & $1700-1750$ & 1795 & 1768 & 1688 & $2146 \pm 16$ \\
\hline & $1900^{(* * *)}$ & $(1862-1975)$ & 1870 & 1808 & 1809 & $2314 \pm 20$ \\
\hline & $2040^{(*)}$ & $2031-2065$ & 1910 & - & 1936 & $2334 \pm 22$ \\
\hline & - & - & 1950 & - & 1969 & $2401 \pm 17$ \\
\hline \multirow{4}{*}{$\frac{5}{2}^{+}$} & $1680^{(* * * *)}$ & $1680-1690$ & 1770 & 1808 & 1723 & $2143 \pm 17$ \\
\hline & $1860^{(* *)}$ & $1820-1960$ & 1980 & - & 1934 & $2352 \pm 22$ \\
\hline & $2000^{(* *)}$ & $1950-2150$ & 1995 & - & 1959 & $2415 \pm 18$ \\
\hline & - & - & 1995 & - & 2120 & $2943 \pm 68^{*}$ \\
\hline \multirow{3}{*}{$\frac{7}{2}^{+}$} & $1990^{(* *)}$ & $(1920-2155)$ & 2000 & - & 1989 & $2481 \pm 20$ \\
\hline & - & - & 1995 & - & 2190 & $2900 \pm 57$ \\
\hline & - & - & & - & 2365 & \\
\hline$\frac{9}{2}^{+}$ & $2220^{(* * * *)}$ & $2200-2300$ & 2345 & & 2221 & \\
\hline$\frac{13}{2}^{+}$ & $2700^{(* *)}$ & $(2570-3100)$ & 2820 & & 2616 & \\
\hline \multirow{3}{*}{$\frac{1}{2}^{-}$} & $1535^{(* * * *)}$ & $1525-1545$ & 1460 & 1527 & 1435 & $1707 \pm 21$ \\
\hline & $1650^{(* * * *)}$ & $1645-1670$ & 1535 & 1671 & 1660 & $1860 \pm 27$ \\
\hline & $1895^{(* *)}$ & $(1860-2260)$ & $\begin{array}{l}1945 \\
2030\end{array}$ & 1882 & $\begin{array}{l}1901 \\
1918\end{array}$ & $2357 \pm 113$ \\
\hline \multirow{4}{*}{$\frac{3}{2}^{-}$} & $1520^{(* * * *)}$ & $1515-1525$ & 1495 & 1527 & 1476 & $1811 \pm 22$ \\
\hline & $1700^{(* * *)}$ & $1650-1750$ & 1625 & 1671 & 1606 & $1889 \pm 21$ \\
\hline & $1875^{(* * *)}$ & $1820-1920$ & 1960 & 1882 & 1926 & $2513 \pm 54$ \\
\hline & $2120^{(* *)}$ & $(1980-2210)$ & $\begin{array}{l}2055 \\
2095\end{array}$ & & $\begin{array}{l}1959 \\
2070\end{array}$ & $2673 \pm 20$ \\
\hline \multirow[b]{2}{*}{$\frac{5}{2}^{-}$} & $1675^{(* * * *)}$ & $1670-1680$ & 1630 & 1671 & 1655 & $1987 \pm 17$ \\
\hline & $2060^{(* *)}$ & $(1900-2260)$ & $\begin{array}{l}2080 \\
2095\end{array}$ & & $\begin{array}{l}1970 \\
2104\end{array}$ & $2486 \pm 24$ \\
\hline$\frac{7}{2}$ & $2190^{(* * * *)}$ & $2100-2200$ & 2090 & & 2015 & $2635 \pm 22$ \\
\hline$\frac{9}{2}^{-}$ & $2250^{(* * * *)}$ & $2200-2350$ & 2215 & & 2212 & \\
\hline$\frac{11}{2}^{-}$ & $2600^{(* * *)}$ & $2550-2750$ & 2600 & & $\begin{array}{l}2425 \\
2600\end{array}$ & \\
\hline
\end{tabular}

Figure 1.3: Table shows measured masses of resonances and the corresponding model predictions. Column four shows the predicted states and their masses for the constituent model referenced above, likewise column 5 displays the same info for the diquark model, column 6 the relativistic model, and finally column 7 shows the information for the lattice calculations[30]. 


\subsection{Photoproduced Proton Spectra}

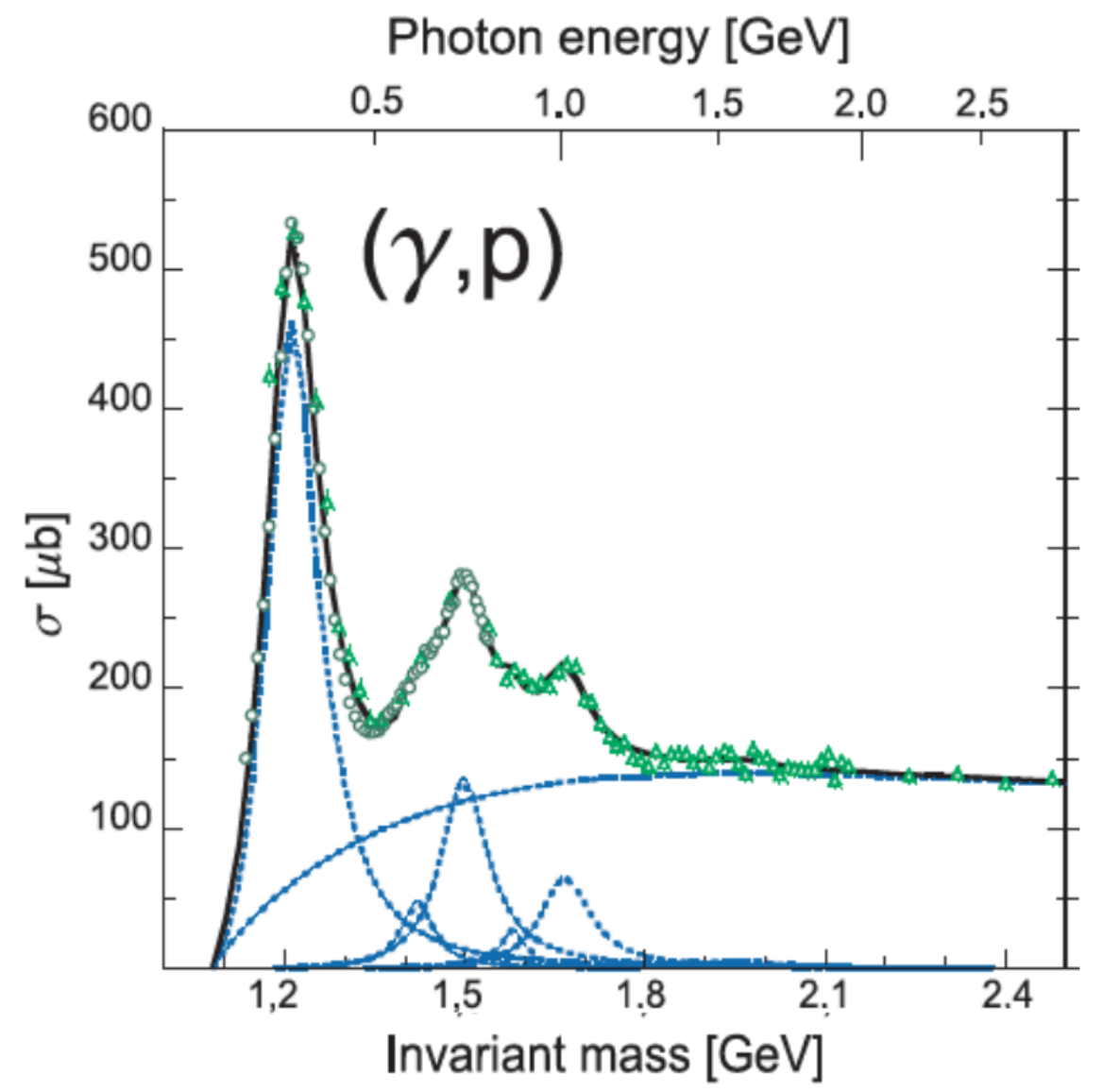

Figure 1.4: Plot shows the total cross section of $\gamma p \rightarrow N^{*}$. Certain well established resonances are shown beneath [34][35]. It is clear that some of these could not be extracted from the spectrum [36].

Observing a resonance directly is not possible due to their very short lifetimes $(\sim$ $10^{-23} \mathrm{~s}$ ). This also results in peaks in the spectra which are very wide. On its own this would not prove a problem, but as was described above, there are many predicted resonances and they have very similar masses. This results in spectra which are impossible to disentangle just by studying them directly. Figure 1.4 shows this for the total cross section of protons excited using a photon probe.

Therefore, we need something else to help establish which resonances exist and which do not. One tool is to measure polarization observables associated with the excitation of the nucleon and the corresponding produced mesons. The polarization observables are also referred as spin observables, which gives a clearer indication as to 
what they are. They are variables which describe the effect that the transition mechanisms have on the particles produced via photoproduction. Hence, they are sensitive to which resonances exist and which do not. However, individial measurements cannot establish the existence of a resonance. They need to be used in conjunction with model predictions and also need to be combined in a meaningful way to try and distentangle all the potential resonances and also the non-resonant transition mechanism.

This is done using Partial Wave Analysis (PWA); different contributions are separated by analysing angular momentum and separating out different wave types, e.g. separating $\mathrm{P}$ waves from $\mathrm{D}$ waves. There are a number of groups who work solely on PWA; the Scattering Analysis Interactive Dial-in (SAID) group, Mainz Unitary Isobar Model (MAID), the Gießen group, and the Bonn-Gatchina group are the most important contributors with the latter two being particular important due to their multiparticle final state analyses [37][38][39][40].

The aim of this experiment is to measurement one of the polarization observables for the reaction channel $\gamma p \rightarrow \pi^{+} \pi^{-} p$ and more specifically via $\gamma p \rightarrow \rho^{0} p$ and $\gamma p \rightarrow f^{0} p$. The observable which will be measured is the beam asymmetry. This measures the reaction mechanism's sensitivity to the orientation of the electric vector of the incident photon beam and can help highlight what processes are occurring. This analysis is part of a wider program at the Jefferson Labratory Facility (JLab) called the $\mathrm{N}^{*}$ Program. An overview of the results to date and the on-going experiments can be read in [41] and [42].

The following chapter will describe the formalism and theory pertinent to the analysis undertaken for this thesis, describing in more detail how these polarization observables relate to the transition mechanisms and discussing how to measure these observables. 


\section{Chapter 2}

\section{Two Pion Photoproduction}

The majority of meson photoproduction analyses have been focused on single pion production. In the lower end of the resonant region, $<1.7 \mathrm{GeV}$ centre-of-mass, single pion production is the main reaction channel. However, at greater energies two pion production becomes the principal reaction channel; it is this region that has the most ambiguity [43]. It is also important to study two pion photoproduction because theoretical calculations suggest that the missing resonances have a higher probability of coupling with two pion photoproduction than single pion production [44][31][45].

Some two pion production has been undertaken previously but it was mostly performed using a pion beam $(\pi N \rightarrow \pi \pi N)$ [46]. A small amount of photoproduced double pion experiments were performed in the sixties and the seventies but mainly above the resonant region [47][48]. Recently, more two pion photoproduction analyses have been undertaken focusing on the resonant region [36][49][50].

The analysis of this thesis and the corresponding theory to be discussed will deal with the reaction channel $\gamma p \rightarrow \pi^{+} \pi^{-} p$ with a focus on the intermediate state of the $\rho^{0}$ meson. 


\subsection{Pions}

Pions are the lightest of the mesons and come in three varieties: either positively charged $\left(\pi^{+}\right)$, negatively charged $\left(\pi^{-}\right)$, or uncharged $\left(\pi^{0}\right)$. They are composed of the following quark anti-quark compositions: $u \bar{d}, d \bar{u}$, and $\frac{u \bar{u}-d \bar{d}}{\sqrt{2}}$, respectively. They are spinless particles and have negative parity, therefore are so-called pseudoscalar mesons $\left(J^{P}=0^{-}\right)$. The mass of the charged pions is $139.570 \mathrm{MeV} / \mathrm{c}^{2}$ and that of the neutral pion is $134.977 \mathrm{MeV} / \mathrm{c}^{2}$.

The relatively long lifetime of the charged particles $\left(2.6033 \pm 0.0005 \times 10^{-8} \mathrm{~s}\right) \mathrm{com}-$ pared with that of the neutral pion $\left(8.4 \pm 0.6 \times 10^{-17} \mathrm{~s}\right)$ means that they can be experimentally detected. Therefore, considering the final state particles to be detected in this analysis are $\pi^{+} \pi^{-} p$, we can have a fully exclusive measurement of the reaction, excluding any geometric restrictions of the detector [51].

\subsection{The $\rho^{0}$ Meson}

The dominant intermediate state of $\pi^{+} \pi^{-}$photoproduction is the $\rho^{0}$ meson, which almost exclusively decays via $\pi^{+} \pi^{-}$with a branching ratio of $\sim 100 \%$. The rho mesons have the same quark compositions as the pions, but they also are spin 1 particles. In addition, the $\rho^{0}$ meson has a mass of $775.26 \mathrm{MeV} / \mathrm{c}^{2}$, which is significantly higher than the mass of the pions; the difference between the masses is still a contemporary problem with the spin difference being the presumed cause [52]. The $\rho^{0}$ meson has the following quark composition: $\frac{u \bar{u}-d \bar{d}}{\sqrt{2}}$. It has a mean lifetime is $\sim 4.5 \times 10^{-24} \mathrm{~s}$, conversely this means that it has a large width, $149.5 \mathrm{MeV}$. This adds difficulty when trying to separate a rho signal from other contributions in comparison to other mesons such as the $\phi$ meson which has a width of $4.27 \mathrm{MeV}$. The $\rho^{0}$ meson has negative parity so, along with its spin 1 nature, it is part of the vector meson multiplet $\left(J^{P}=1^{-}\right)$. Therefore, the following section will discuss the theory of polarization observables in terms of vector meson photoproduction, which in any case is a more generalised form 
of the pseudoscalar treatment.

\subsubsection{The $f^{0}$ Meson}

In certain kinematic regions another mesonic state is apparent in the invariant mass distributions of the two charged pions. This is the $f^{0}$ meson, which is a scalar meson canditate, $\left(J^{P}=0^{+}\right)$. Its branching ratios have not been quantified yet. However, it is clear that that $\pi^{+} \pi^{-}$channel is the dominant branch with the other two known modes being $K \bar{K}$ amd $\gamma \gamma$. The kaon decay mode is interesting as it shows that strangeness is important to the $f^{0}$ meson and suggests that the simple quark-antiquark model of mesons might not fit well for this scalar meson. In fact, it has been postulated that the light scalar mesons might be diquark-antidiquark structures and some theoretical calculations seem to support this [53][54]. Whether it is an exotic particle or not, its characterstics are not well known. The Particle Data Group give a mass of 990 \pm 20 $\mathrm{MeV}$ and a width of $40-100 \mathrm{MeV}$ [51]. So any information about this particle is of interest. The spin 0 nature of the $f^{0}$ means that the same treatment of polarization information in pseudo-scalar mesons can be used. The beam asymmetry will be measured for the $f^{0}$ alongside the $\rho^{0}$. 


\subsection{Vector Meson Photoproduction Kinematics}

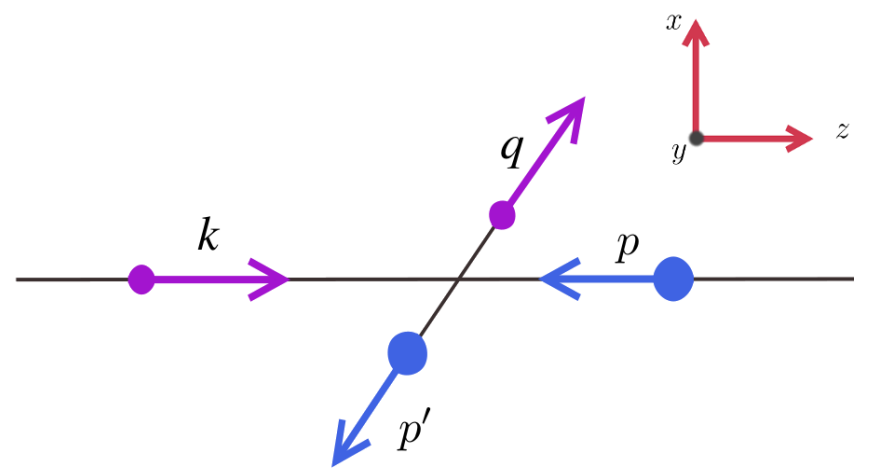

Figure 2.1: Diagram of the kinematics involved in a scattering reaction as viewed in the centre-of-mass frame, where $k, q, p, p^{\prime}$ are the four-momenta of the incident photon, the produced meson, the target nucleon, and the scattered nucleon, respectively [55].

The kinematics of the reaction as observed in the centre-of-mass frame are shown in figure 2.1. This process is described mathematically by the scattering matrix, $S$ [56]:

$$
S=i(2 \pi)^{4} \delta\left(k+p-q-p^{\prime}\right) T
$$

Where:

- $k$ is the four-momentum of the incident photon.

- $p$ is the four-momentum of the target nucleon.

- $p^{\prime}$ is the four-momentum of the recoil nucleon.

- $q$ is the four-momentum of the produced $\rho^{0}$ meson.

- $T$ is the transition matrix:

$$
T=\frac{1}{\sqrt{(2 \pi)^{3} \cdot 2 k}}<\rho^{0} N^{\prime}\left|\epsilon_{\mu} \cdot J_{\mu}\right| N>
$$


Where $\epsilon_{\mu}$ is the photon polarization vector and $J_{\mu}$ is the electromagnetic current. It is the transition matrix where resonant contributions are combined with other reaction channels.

It is conventional to discuss these sort of reactions in terms of the Mandelstam variables; a set of three Lorentz-invariant variables [57]:

$$
\begin{aligned}
& s=\left(q+p^{\prime}\right)^{2} \\
& t=\left(p-p^{\prime}\right)^{2} \\
& u=(p-q)^{2}
\end{aligned}
$$

It is clear from their definition that any of the above Lorentz-variables can be expressed in terms of the other two. Therefore, we describe the scattering proccess in terms of two of these Mandelstam variables. Typically these are $s$ and $t . s$ is equal to the invariant mass squared of the inital state particles (or final) in the centre-of-mass frame $\left(W^{2}\right)$. As the target in our experiment is stationary, this is equivalent to the energy of the incident photon beam which is a very tangible metric to parameterise our analysis. $t$ is the momentum transfer of the scattering process. This is equivalent to the cosine of the polar angle when $s$ is at a constant value. This too is a sensible value to use for separating our data for analysis as it is an appreciable physical value [58]. It is for these reasons that we divide and display our analysis in terms of $\mathrm{W}^{2}$ and $\cos \theta$ ranges. 


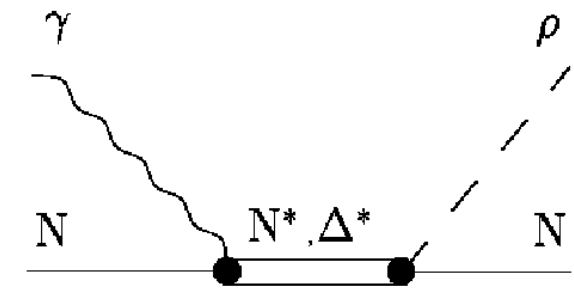

s-channel baryon exchange

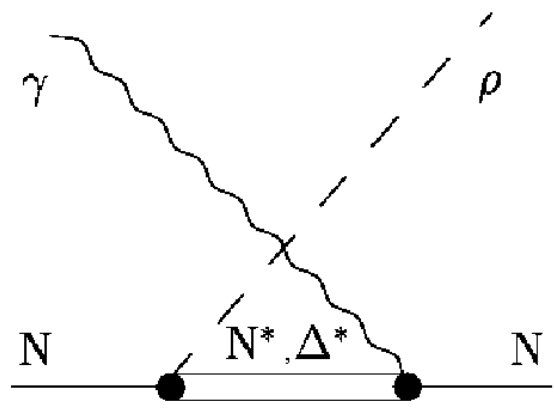

u-channel baryon exchange

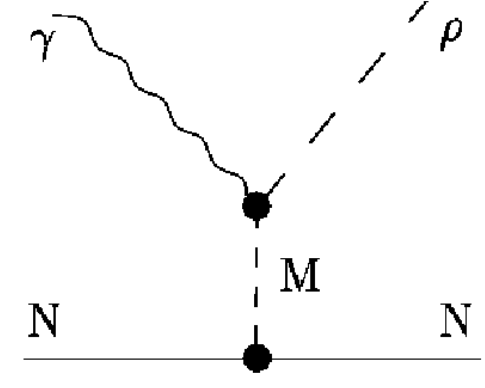

t-channel meson exchange

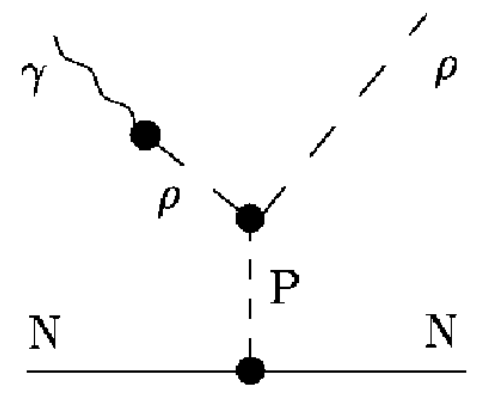

diffractive scattering via pomeron exchange

Figure 2.2: Born diagrams of the different processees which contribute to the photoproduction of the $\rho^{0}$ [59].

The Mandelstam variables are also used for naming different reaction mechanism involved in the photoproduction of the $\rho^{0}$ meson as shown figure 2.2. This is because the respective channels are predominantly dependent on the kinematic used for naming each channel. The channels on the right hand side of the diagram show the diffractive channels; the first shows diffractive scattering via a meson exchange and the second shows it for the case where the intermidiate virtual particle absorbed is the pomeron, a theoretical trajectory. In the diffractive case of photoproduced mesons, the hadron target dissociates into a multi-particle final state due to the exchanging of a universal trajectory so that the photons energised is absorbed by the hadron. The distinction between diffractive processes and resonant processes is the former is the result of the hadron dissociating, and the latter is the result of the hadron being excited to some intermediate resonance and then decaying into a multi-particle final state. [60]. The s-channel and u-channel describe the processes where intermediate resonances exist 
and are thus the process which are of interest here [61].

\subsection{Helicity Amplitudes and Polarization Observables}

The basic amplitude for vector meson photoproduction is given by [62]:

$$
\mathscr{F} \equiv\left\langle\boldsymbol{q} \lambda_{V} \lambda_{2}|T| \boldsymbol{k} \lambda \lambda_{1}\right\rangle
$$
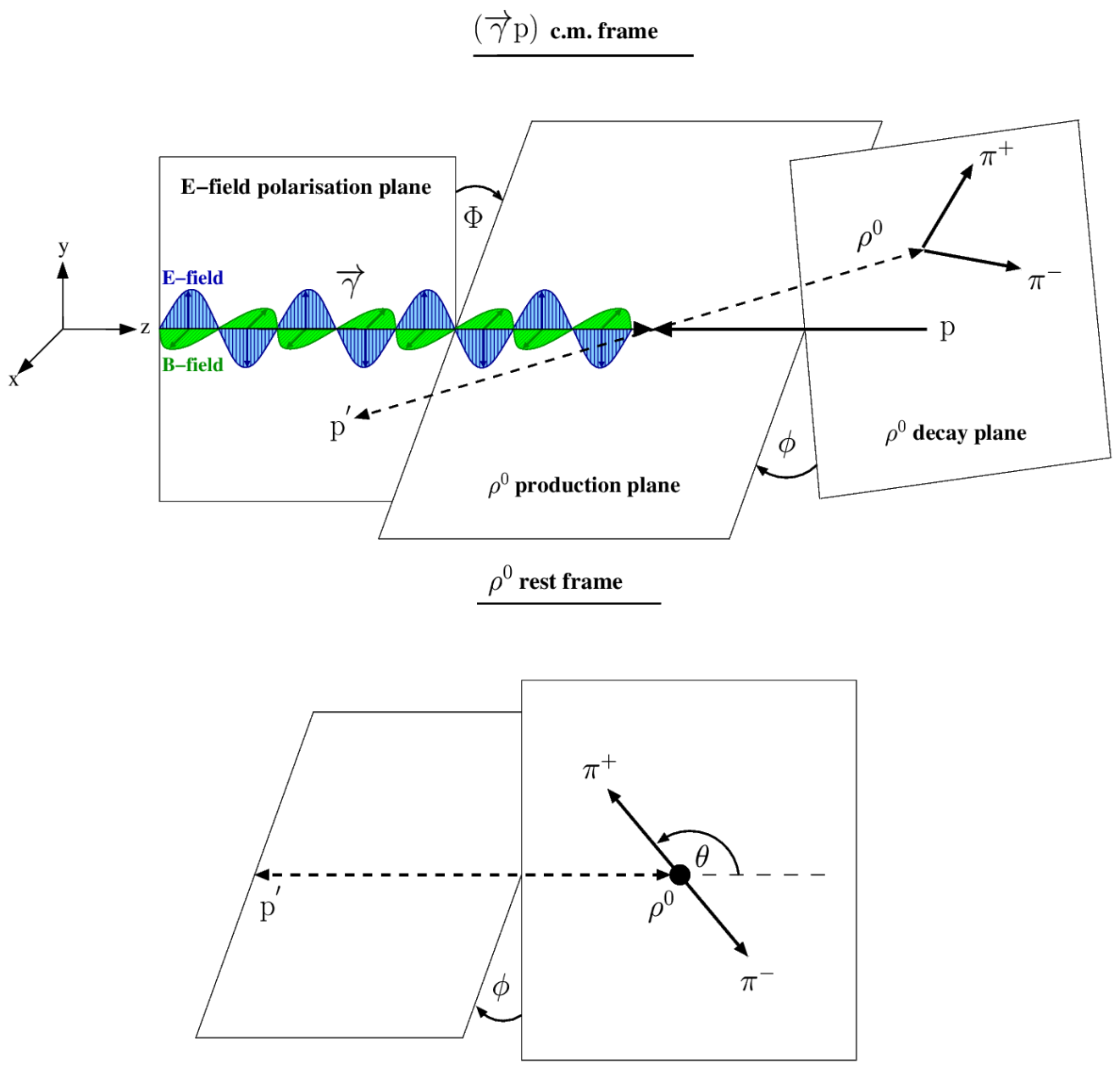

Figure 2.3: A diagram showing the different planes and angles involved in the photoproduction of $\rho^{0}$ mesons [59].

where $\lambda$ represents the helicity state of the of the particles involved: subscript $\mathrm{V}$ denoting that of the meson, 1 and 2 representing the initial and final helicity state of 
the nucleon, respectively, and no subscript that of the incident photon. $k$ is the initial relative momenta of the system and $\boldsymbol{q}$ is that of the final state. The helicity state of the photon is \pm 1 describing the two possible helicity states it can be. The helicity states of the target nucleon are $\pm \frac{1}{2}$ and the helicity states of the vector meson are $\pm 1,0$. The final state baryon, which is a nucleon in our experiment, depends on its spin: which happens to be the same as for the target. For real photons the amplitude represents 24 complex numbers $(3 * 2 * 2 * 2)$. Due to parity invariance there are 12 relations between these amplitudes and so we end up with 12 independent complex helicity amplitudes or 24 real numbers (for each energy and angle). These helicity amplitudes are expressed as follows:

$$
\left\langle\boldsymbol{q} \lambda_{V} \lambda_{2}|T| \boldsymbol{k} \lambda \lambda_{1}\right\rangle \rightarrow H_{a \lambda_{V}}(\theta)
$$

$a=1, \ldots, 4$ and $\lambda_{V}= \pm 1,0$ : by taking $\lambda=0$ we simplify this down to the standard four amplitudes used to describe pseudo-scalar photoproduction. The vector meson amplitude can be expressed in helicity space by the following matrix:

$$
\mathscr{F}=\left[\begin{array}{cccc}
H_{21} & H_{11} & H_{3-1} & -H_{4-1} \\
H_{41} & H_{31} & -H_{1-1} & H_{2-1} \\
H_{20} & H_{10} & -H_{30} & H_{40} \\
H_{40} & H_{30} & H_{10} & -H_{20} \\
H_{2-1} & H_{1-1} & H_{31} & -H_{41} \\
H_{4-1} & H_{3-1} & -H_{11} & H_{21}
\end{array}\right]
$$

Where the Jacob-Wick parity allows us to describe the transition matrix as: [63]:

$$
\left\langle\boldsymbol{q} \lambda_{V} \lambda_{2}|T| \boldsymbol{p} \lambda \lambda_{1}\right\rangle=(-1)^{\Lambda_{f}-\Lambda_{i}}\left\langle\boldsymbol{q}-\lambda_{V}-\lambda_{2}|T| \boldsymbol{p}-\lambda-\lambda_{1}\right\rangle
$$

$\Lambda_{i} \equiv \lambda-\lambda_{1}$ and $\Lambda_{f} \equiv \lambda-\lambda_{1}$. The rows of the matrix are labelled by the final states helicities and the the columns by the intial state (where the half spin states of the nucleons have been taken to be integers so as to make the indexing clearer). The 
helicity amplitudes in $\mathscr{F}$ are:

$$
\begin{aligned}
& H_{1 \lambda_{V}} \equiv\left\langle\lambda_{V}, \lambda 2=+\frac{1}{2}|T| \lambda=1, \lambda_{1}=-\frac{1}{2}\right\rangle \\
& H_{2 \lambda_{V}} \equiv\left\langle\lambda_{V}, \lambda 2=+\frac{1}{2}|T| \lambda=1, \lambda_{1}=+\frac{1}{2}\right\rangle \\
& H_{3 \lambda_{V}} \equiv\left\langle\lambda_{V}, \lambda 2=-\frac{1}{2}|T| \lambda=1, \lambda_{1}=-\frac{1}{2}\right\rangle \\
& H_{4 \lambda_{V}} \equiv\left\langle\lambda_{V}, \lambda 2=-\frac{1}{2}|T| \lambda=1, \lambda_{1}=+\frac{1}{2}\right\rangle
\end{aligned}
$$

For the general case, for all spin observables for vector meson photoproduction, general spin observables, $\Omega$ can be written as follows:

$$
\Omega=\frac{\operatorname{Tr}\left[\mathscr{F}\left(A_{\gamma} A_{N}\right) \mathscr{F}^{\dagger}\left(B_{V} B_{N^{\prime}}\right]\right.}{\operatorname{Tr}\left[\mathscr{F} \mathscr{F}^{\dagger}\right]}
$$

where the trace is over the spin-space helicity quantum numbers. Interference occurs only at the quantum level for the basic two-body interaction. The matrices have the dimensions $\mathscr{F}(6 \times 4), A_{\gamma} A_{N}(4 \times 4), \mathscr{F}^{\dagger}(4 \times 6)$, and $B_{V} B_{N^{\prime}}(6 \times 6)$ [62]. There are two degrees of freedom on the photon with respect to spin. This is expressed by $A_{\gamma}$ which is the standard $2 \times 2$ Hermitian spin matrices $\left(1, \boldsymbol{\sigma}_{\gamma}\right)$ and includes the Stoke vector, $\mathbf{P}_{S}$ to account for the vector nature of the photon. $A_{N}$ is that of the target nucleon, $B_{N^{\prime}}$ that of the recoil nucleon: both are a $2 \times 2$ matrix. The vector meson matrix, $B_{V}$, is a $3 \times 3$ matrix.

There are density matrices which describe the polarisation of each particle in this production process and these are associated with the above ensemble average describing the general spin observable. The one pertinent to our interests is the density matrix for the incident photon:

$$
\rho_{\gamma}=\frac{\boldsymbol{I}_{\gamma}}{2}\left(1+\gamma+\boldsymbol{\sigma}_{\gamma} \cdot \boldsymbol{P}_{S}\right)
$$

This equation re-emerges again later in a different frame as 2.21 .

When only the incident photon is polarized equation 2.15 simplifies and gives us 
the polarized beam asymmetry, $\Sigma$, (referred to as the analysing power of the beam). This is expressed as follows:

$$
\Sigma_{x}=\frac{\operatorname{Tr}\left[\mathscr{F} \sigma_{x}^{y} \mathscr{F}^{\dagger}\right]}{\operatorname{Tr}\left[\mathscr{F} \mathscr{F}^{\dagger}\right]}
$$

If we define $\sigma^{B, T ; R, V}$ for the cross section $d \sigma / d \Sigma$ where the superscripts (B,T;R,V) denote the polarizations of photon beam, target nucleon; recoil nucleon, produced vector-meson) then the physical of meaning of $\Sigma_{x}$ becomes clear through the relation:

$$
\Sigma_{x}=\frac{\sigma^{\|, U ; U, U}-\sigma^{\perp, U ; U, U}}{\sigma^{\|, U ; U, U}+\sigma^{\perp, U ; U, U}}
$$

where the superscript $U$ refers to an unpolarized particle and $\|(\perp)$ corresponds to a photon linearly polarized along the $\boldsymbol{x}(\boldsymbol{y})$ axis [64].

\subsection{Deriving Angular Distibrution}

The following method of analysing the angular distribution is called the Schilling method [65]. We start by considering the spin and helicity states of the photon. Linearly polarised photon states can be described by a linear combination of left-handed $(J=-1)$ and right-handed $(J=1)$ circularly polarised spin states. The resulting helicity state is dependent on the spin-helicity relationship [66]:

$$
H=\frac{\boldsymbol{\sigma} \cdot \boldsymbol{p}}{E_{\gamma}|\boldsymbol{\sigma}|}
$$

For the sake of clarity the angular distribution of a vector meson will be derived and applied to the $\rho^{0}$ case retrospectively as confusion with the standard notation is more than likely. In order to derive the angular distributions we need the spin density matrix of the decaying vector meson, which is related to the photon spin density matrix as shown here:

$$
\rho(V)=T \rho(\gamma) T^{\dagger}
$$


$\mathrm{T}$ is the transition amplitude as above of for the rho meson in the helicity formalism and $\rho(\gamma)$ can be expressed as follows:

$$
\rho(\gamma)=\frac{1}{2} \boldsymbol{I}+\boldsymbol{P}_{\gamma} \cdot \boldsymbol{\sigma}
$$

where, for linear polarisation:

$$
\boldsymbol{P}_{\gamma}=P_{\gamma}(-\cos 2 \Phi,-\sin 2 \Phi, 0)
$$

$\Phi$ is the angle between thr production plane the polarisation vector of the the photon and $P_{\gamma}$ is the degree of linear polarisation of the photon $\left(0 \leqslant P_{\gamma} \leqslant 1\right)$. Using 2.20 and 2.21 and the helicity-amplitude formalism we can show the dependence of the polarisation vector $\boldsymbol{P}_{\gamma}$ of the density matrix $\rho(V)$ :

$$
\rho(V)=\rho^{o}+\sum_{i=1}^{3} P_{\gamma}^{\alpha} \rho^{\alpha}
$$

Where $P_{\gamma}^{\alpha}$ are the components of the polarisation vector, $\boldsymbol{P}_{\gamma}$, and the $\rho^{\alpha}$ are hermitian matrices:

$$
\begin{aligned}
\rho_{\lambda_{V} \lambda_{V}^{\prime}}^{0} & =\frac{1}{2 N} \sum_{\lambda_{V} \lambda_{N^{\prime}} \lambda_{N}} T_{\lambda_{V} \lambda_{N^{\prime}}, \lambda_{\gamma} \lambda_{N}} T_{\lambda_{V}^{\prime} \lambda_{N^{\prime}}, \lambda_{\gamma} \lambda_{N}}^{*} \\
\rho_{\lambda_{V} \lambda_{V}^{\prime}}^{1} & =\frac{1}{2 N} \sum_{\lambda_{V} \lambda_{N^{\prime}} \lambda_{N}} T_{\lambda_{V} \lambda_{N^{\prime}},-\lambda_{\gamma} \lambda_{N}} T_{\lambda_{V}^{\prime} \lambda_{N^{\prime}}, \lambda_{\gamma} \lambda_{N}}^{*} \\
\rho_{\lambda_{V} \lambda_{V}^{\prime}}^{2} & =\frac{i}{2 N} \sum_{\lambda_{V} \lambda_{N^{\prime}} \lambda_{N}} \lambda_{\gamma} T_{\lambda_{V} \lambda_{N^{\prime}},-\lambda_{\gamma} \lambda_{N}} T_{\lambda_{V}^{\prime} \lambda_{N^{\prime}}, \lambda_{\gamma} \lambda_{N}}^{*}
\end{aligned}
$$

The density matrix is related to the decay angular distribution:

$$
W(\cos \theta, \phi)=M \rho(V) M^{\dagger}
$$

$\mathrm{M}$ is the decay amplitude and $\theta$ and $\phi$ are the polar and azimuthal angles of the detected $\pi^{+}$in the helicity frame. The helicity frame is defined as the $\rho^{0}$ rest frame with 
the z-axis orienated in the opposite direction of the recoil nucleon [67]. Other reference frames can be used such as the Gottfried-Jackson frame or the Adair frames. These differ only in terms of the orientation of the z-axis and give different benefits for analysis [68][69]. From here we move on to the $\rho^{0}$-meson decay distribution:

$$
W(\cos \theta, \phi, \Phi)=W^{0}(\cos \theta, \phi)-P_{\gamma} \cos 2 \Phi W^{1}(\cos \theta, \phi)-P_{\gamma} \sin 2 \Phi W^{2}(\cos \theta, \phi)
$$

where

$$
W^{0}(\cos \theta, \phi)=\frac{3}{4 \pi}\left(\frac{1}{2}\left(1-\rho_{00}^{0}\right)+\frac{1}{2}\left(3 \rho_{00}^{0}-1\right) \cos ^{2} \theta-\sqrt{2} R e \rho_{10}^{0} \sin 2 \theta \cos \phi-\rho_{1-1}^{0} \sin ^{2} \theta \cos 2 \phi\right.
$$

$$
W^{1}(\cos \theta, \phi)=\frac{3}{4 \pi}\left(\rho_{11}^{1} \sin ^{2} \phi+\rho_{00}^{1} \cos ^{2} \theta-\sqrt{2} \rho_{10}^{1} \sin 2 \theta \cos \phi-\rho_{1-1}^{1} \sin ^{2} \theta \cos 2 \phi\right)
$$

$$
W^{2}(\cos \theta, \phi)=\frac{3}{4 \pi}\left(\sqrt{2} I m \rho_{10}^{2} \sin 2 \theta \sin \phi+I m \rho_{1-1}^{2} \sin ^{2} \theta \sin 2 \phi\right)
$$

In order to attain a function for extracting the beam asymmetry we integrate over the two angles associated with the pion in the helicity frame angles to leave us with a single angle function [70]. We are left with the following equation:

$$
W(\Phi)=\frac{1}{2 \pi}\left[1-P_{\gamma}\left(2 \rho_{11}^{1}+\rho_{00}^{1}\right) \cos 2 \Phi\right]
$$

Where:

$$
\Sigma=2 \rho_{11}^{1}+\rho_{00}^{1}
$$

With a polarized beam, vector meson photoproduction experiments have the potential for other polarization measurements alongside that of the beam asymmetry so the angular distribution is potentially affected by these. The experiment is sensitive to the tensor polarization of the $\rho^{0}$ and this manifested in dependency of the $\Phi$ distribution on 
the pion's azimuthal angle in the helicity frame [71]. This is discussed in more detail in section 6.4.

\subsection{Summary}

Polarization observables give us measurements which are sensitive to the resonances we seek. In this chapter the relationship between the tranisiton matrix, which includes the details of the processes which are involved in vector meson photoproduction and where the addition or subtraction of resonances in theoretical predictions take place, and the polarization observables have been shown. This relationship shows how the obervables which are experimentally measurable relate back to the theoretical predictions.

Polarization observables can often seem quite removed from the overarching goal of establishing the nature of hadron spectra and so it is necessary to try and maintain an understanding of how the experimental side of hadron sprectroscopy relates to the theoretical. Also, show in this chapter in this relationship between the SDMEs and polarization observables. This is necessary as the common method of using angular distribution of the photoproduced mesons' decay products is derived using SDMEs, rather than polarization obversables. SDMEs and polarization observables are intrinsically linke; both are directly associated with helicity amplitudes. The advantage of polarization observables over SDMEs is that they often relate to physical phenoma in a clearer manner. An example of how these two observables are related is given above in equation 2.33, where the beam asymmetry is shown in terms of SDMEs.

It has been shown theoretically that single polarization observables can be directly sensitive to resonances [72]. However, the most probable scenario is that we attain a measurement which gives information only when combined with other measurements and theoretical predictions. In this analysis we will be focusing on measuring the beam asymmetry for the $\rho^{0}$ meson and the $f^{0}$ meson, along with measurements of the decay pions' asymmetries. 
The $\rho^{0}$ meson is diffractive dominant and therefore a forward angle dominant reaction. This will be shown in the next section when looking at previous cross section measurements. For a purely diffractive reaction the beam asymmetry will be $\sim 1$ as the yield of the parallel orientation will be much greater than that of the perpendicular orientation [73]. It is expected, therefore that when $\cos \theta$ will be $\sim 1$ then also $\Sigma$ will be $\sim 1$ and that the backward angle region is the likeliest place to see resonant behaviour; any deviation from 1 will indicate the other channels, $s$ and $u$, play a greater role [74]. While the beam asymmetry measurement on its own might not shed light on specific details of the resonances involved, it can at highlight different sensitivities in varying kinematic regions.

In the following section results from previous experiments will shown. These include measurements specifically for $\rho^{0}$ photoproduction, but also beam asymmetry measurements for other vector meson, other polarization observables and cross sections measured for the $\rho^{0}$, analysis focused on $f^{0}$ mesons and also some analyses on $\pi^{+} \pi^{-}$photoproduction 


\section{Chapter 3}

\section{Previous Measurements}

A brief overview of the results obtained from previous experiments is given in this chapter.

\section{1 $\rho^{0}$ Photoproduction Results}

Experiments go back to the sixties and seventies when innovated facilities were developed in order to undertake photoproduction experiments with polarazied and unpolarized beams. The Aachen-Berlin-Bonn-Hamburg-Heidelberg-München (ABBHHM) collaboration measured cross sections and differential cross sections across the resonance region. The latter are shown in figure 3.1. They display very distinctive diffractive dominant characterstics with a very high cross section at low $t$ with a steep tail as $t$ increases. Similarly, Ballam et al measured differential cross sections, but also measured SDMEs and beam asymmetries [75][76]. The beam asymmetries are shown in figure 3.2 and are shown alongside other measurements contemporary with Ballam et al, namely from the Deutsches Elektronen-Synchrotron (DESY) and from Cornell [75][76][77][78]. These measurements also suggest the reaction is purely diffractive as the measurements are clustered about 1 , but it must be highlighted that their measurements are only in the forward angle region and do not cover the backward angle at all, the region where other contributions are expected to become more domi- 
nant. Also, they were undertaken with higher energy photon beams and therefore are either above the resonant region or at the upper limit. Another aspect that needs to be highlighted is the dearth of statistics in these experiments.

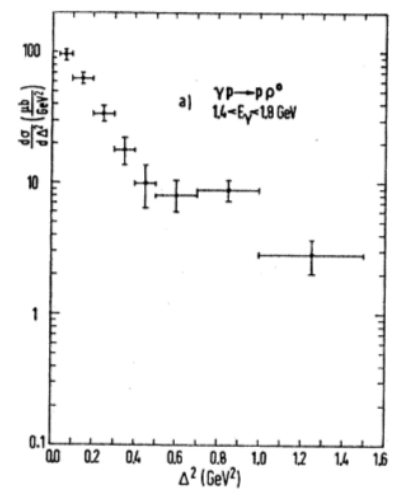

(a)

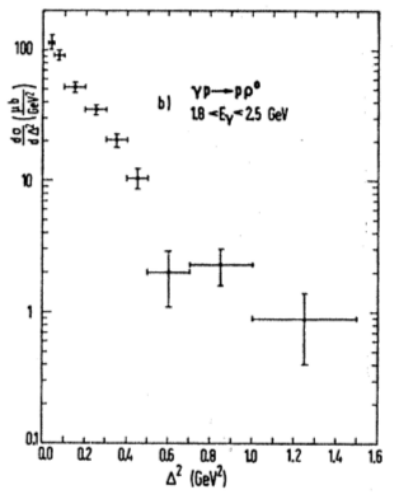

(b)

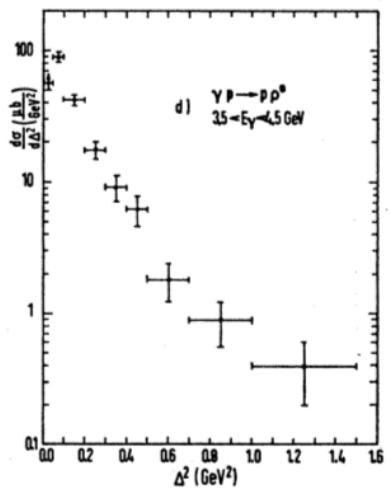

(d)

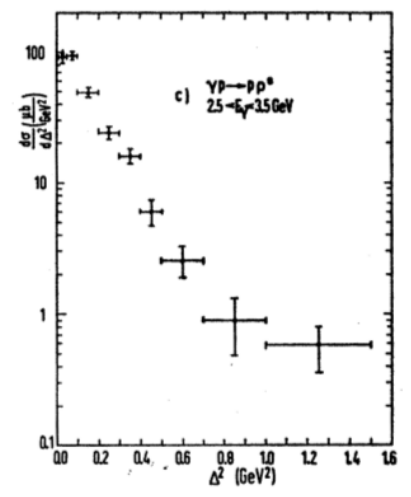

(c)

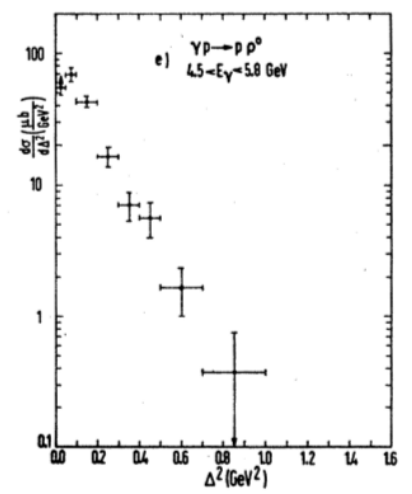

(e)

Figure 3.1: Differential cross sections for five different $E_{\gamma}$ regions as functions of $t^{2}$, the square of the four-momentum transfer from the ABBHHM collaboration [79]. 


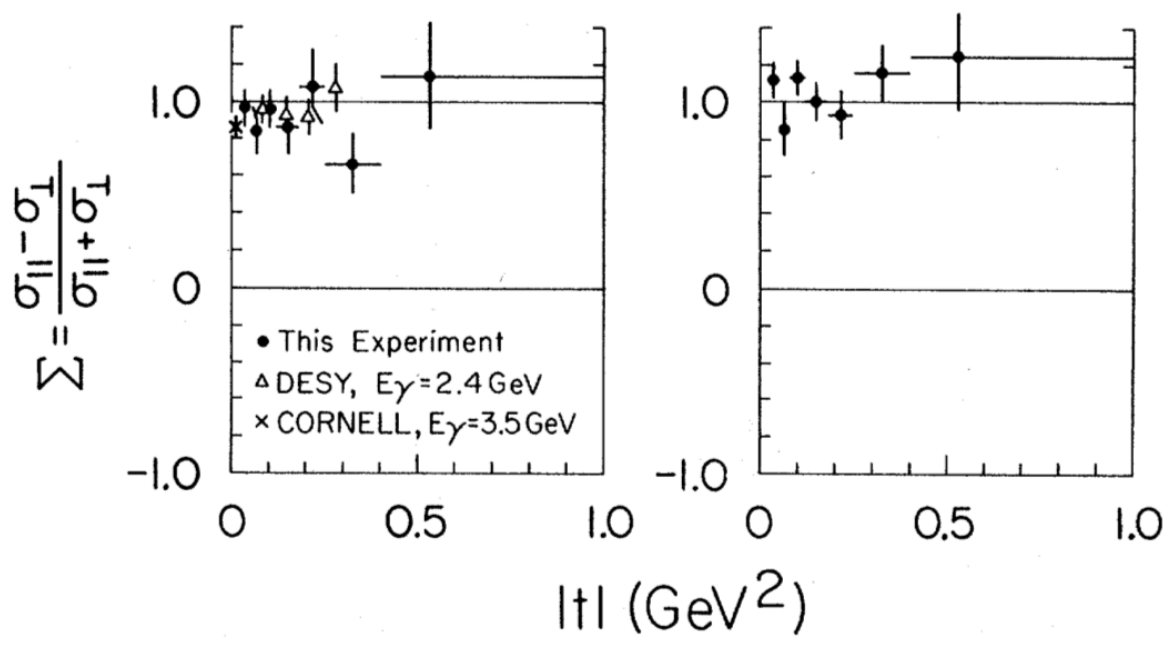

Figure 3.2: Beam asymmetries for photoproduced $\rho^{0}$ mesons as a function of $\mathrm{t}$ for set $E_{g}$ values as shown on the plot. The points labelled 'this experiment' were obtained using a photon beam with an energy of $2.8 \mathrm{GeV}$, the left plot, and $4.7 \mathrm{GeV}$, the righ plot. These energies used are at the upper region of where resonance are found and so the lack of deviation from $\Sigma \sim 1$ is expected as resonant contribution would be minimal with respect to the diffractive channel.

A more recent analysis, similar to the one undertaken here, was done at the Grenoble Anneau Accélérateur Laser (GRAAL), where they measured the beam asymmetry for $\omega$ mesons. $\omega$ mesons are vector mesons and are similar to $\rho$ mesons in terms of their quantum numbers. They have almost identical masses but vary massively in terms of their width; the $\rho^{0}$ meson has a width $\sim 149 \mathrm{MeV}$, whereas the $\omega$ meson has a width of $\sim 8.5 \mathrm{MeV}$ [51]. The results of Ajaka et al are shown in figure 3.3. These are shown to highlight the analytical worth of the beam asymmetry. Their beam asymmetries are mainly negative meaning that the $\mathrm{s}$ and $\mathrm{u}$ channels are dominant, not the diffractive processes [80]. Also, shown are model predictions. It is clear that some model describe the measurements better than others. These deviations are important as they are the main method of deciding whether resonances are present or not. There are also clear structures in these beam asymmetry measurements which intensify as the photon enrgy increases. 


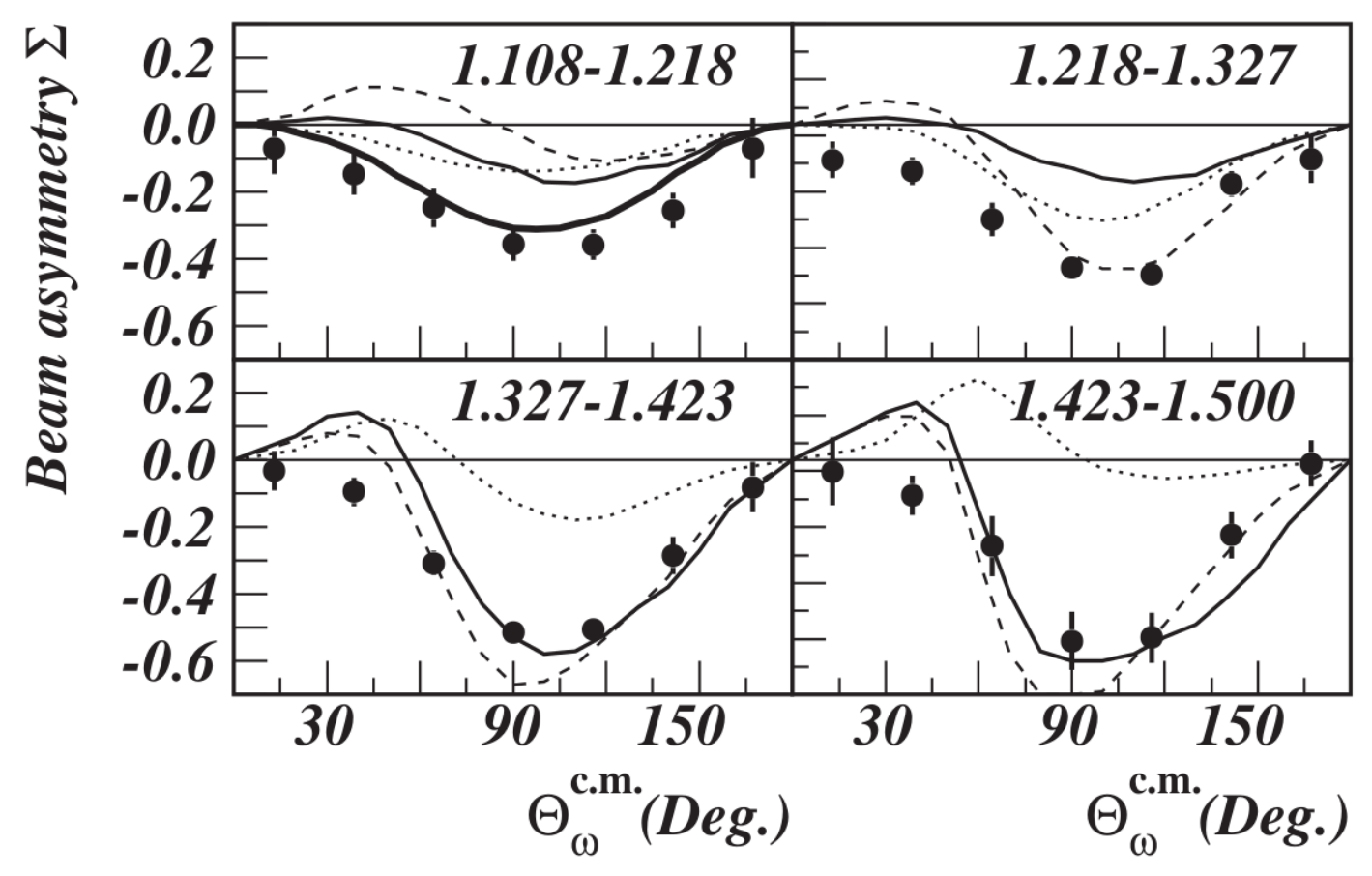

Figure 3.3: Beam asymmetries for photoproduced $\omega$ mesons as a function of $\theta$ in the centre-of-mass frame, equivalanet to $t$, for set $E_{g}$ values as shown on the plot. The solid circles shows the data from GRAAL and the dotted line, dashed line, solid line all show different model prediction [81][38][82]. The negative measured values show that non-diffractive processes are dominant.

The most recent work on $\pi^{+} \pi^{+}$and $\rho^{0}$ photoproduction has been done at the Genovese branch of the Instituto Nazionale di Fisica Nucleare (INFN). They have measured differential cross sections for the $\rho^{0}$ meson and also for the $f^{0}$ by separating the contributions by undertaking a partial wave analysis [50]. These experiments were undertaken at a higher energy range than the experiment used here so we have chosen to use the differential cross sections measured by Klein as a means of cross referencing our measurements in the absence of model predictions to compare [83]. These are shown in figure 3.4. The two model predictions compared to the measurements are for the same model where the dashed lines show the expected values for only diffractive processes and the dotted lines for the resonant processes. It is clear from this that the data agrees well with the diffractive process at the forward angle and that the tailing effect, especially clear in the two lowest energy settings, is at least partially due to the resonant channels becoming more dominant. This gives us a clear indiciation that it is 

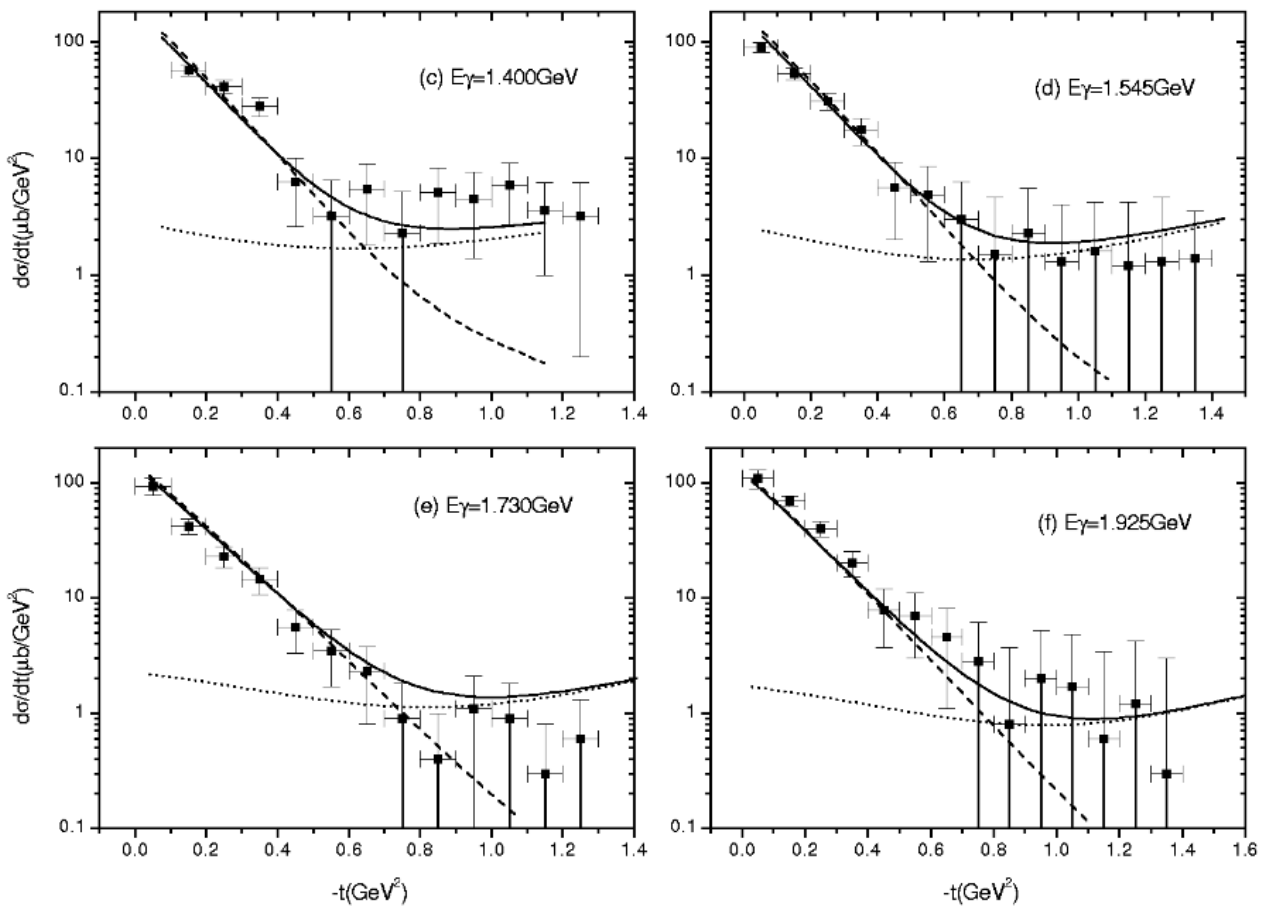

Figure 3.4: Cross sections measurements for $\rho^{0}$ at CLAS as function of $-t$ for different $\mathrm{W}$ regions spanning 1.87-2.12 GeV [83]. The dotted line show model predictions from Zhao using the quark model [84].

worthwhile pursing analyses on the photoproduction of the $\rho^{0}$ meson for the sake of resonant hunting. No other measurements exist, beside those from Battaglieri et al., for the $f^{0}$ meson so we have opted to use those from Battaglieri et al [49]. These are shown in figure 3.5 . The cross sections for the $f^{0}$ are considerably smaller than for the $\rho^{0}$, but they follow the same trend of diffractive dominance at the energy range shown. Any deviation from this simple diffractive slop in the beam asymmetry measurements of the $f^{0}$ will be interesting and will suggest resonant sensitivity for the $f^{0}$.

\subsection{Summary}

A brief overview of the previous measurements has been given. Differential cross sections shown in this section from Battaglieri et al and from Klein will be used when discussing the results of the beam asymmetry extraction. The other measurements shown conform with the diffractive dominant picture of $\rho^{0}$ meson photoproduction, so 


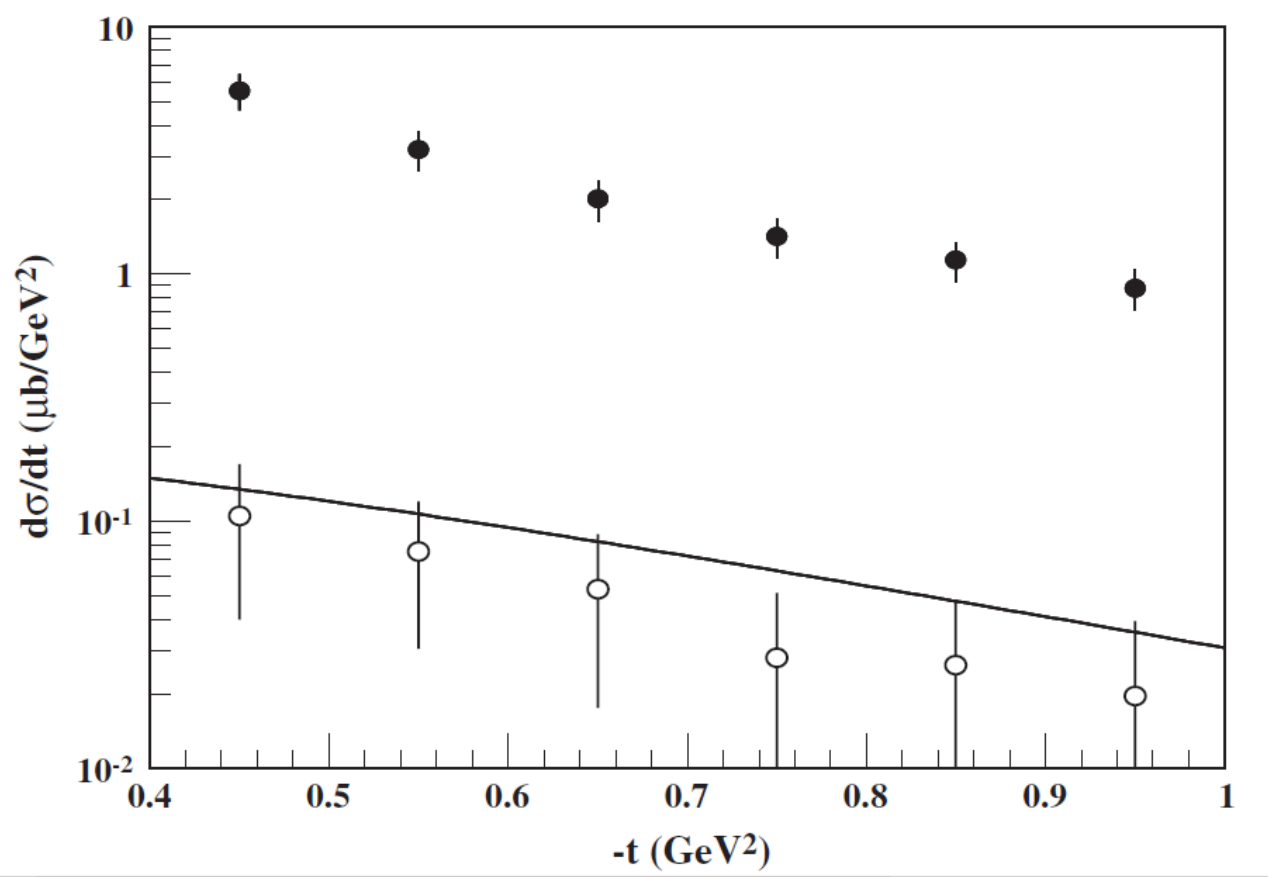

Figure 3.5: Cross sections measured for $f^{0}$ a function of $-t$ for photon energy range $E_{\gamma}=3.0-3.8 \mathrm{GeV}$ [49].

any deviation from that will make for an interesting result.

In the following section the experimental setup used for this analysis will be discussed and the subsequent sections will focus on the analysis. 


\section{Chapter 4}

\section{Experimental Details}

The data used in this analysis was taken at the Jefferson Labratory National Accelerator Facility (JLab) in Virginia, USA beteen the 20th of July and the 1st of September 2005 from the g8b run period in Hall B [85]. The labratory consists of a continuous electron beam accelerator facility and three experimental halls set up in order to undertake different experiments. Both electron and photon beams are utilised for hadronic and nuclear experiments. In this experiment a linearly polarized photon beam was used which was produced using a tagged Bremmstrahlung facility where the incident beam was produced by the Continuous Electron Beam Accelerator Facility (CEBAF). The final state particles were detected by the CEBAF Large Acceptance Spectrometer (CLAS). The electron beam can be distributed to each of the halls simultaneously and at varying currents. The electron beam could run from between $1-6 \mathrm{GeV}$ but is currently being upgraded to reach $12 \mathrm{GeV}$. Details of the accelerator, the tagged Bremstrahlung facilitiy, and the CLAS detector will be discussed in this section.

\subsection{Continuous Electron Beam Accelerator Facility}

The CEBAF is shaped like a racetrack with two long parallel linear accelerators (LINACs) and 2 pairs of circulating arcs at each end, the first pair has 4 arcs and the second has 5 arcs. There is an injector for the initial introduction of electrons into the accelera- 
tor. The accelerator is $1.4 \mathrm{~km}$ in circumference and constitutes 2200 magnets of 58 varieties. A diagram of the accelerator facility can be seen in figure 4.1.

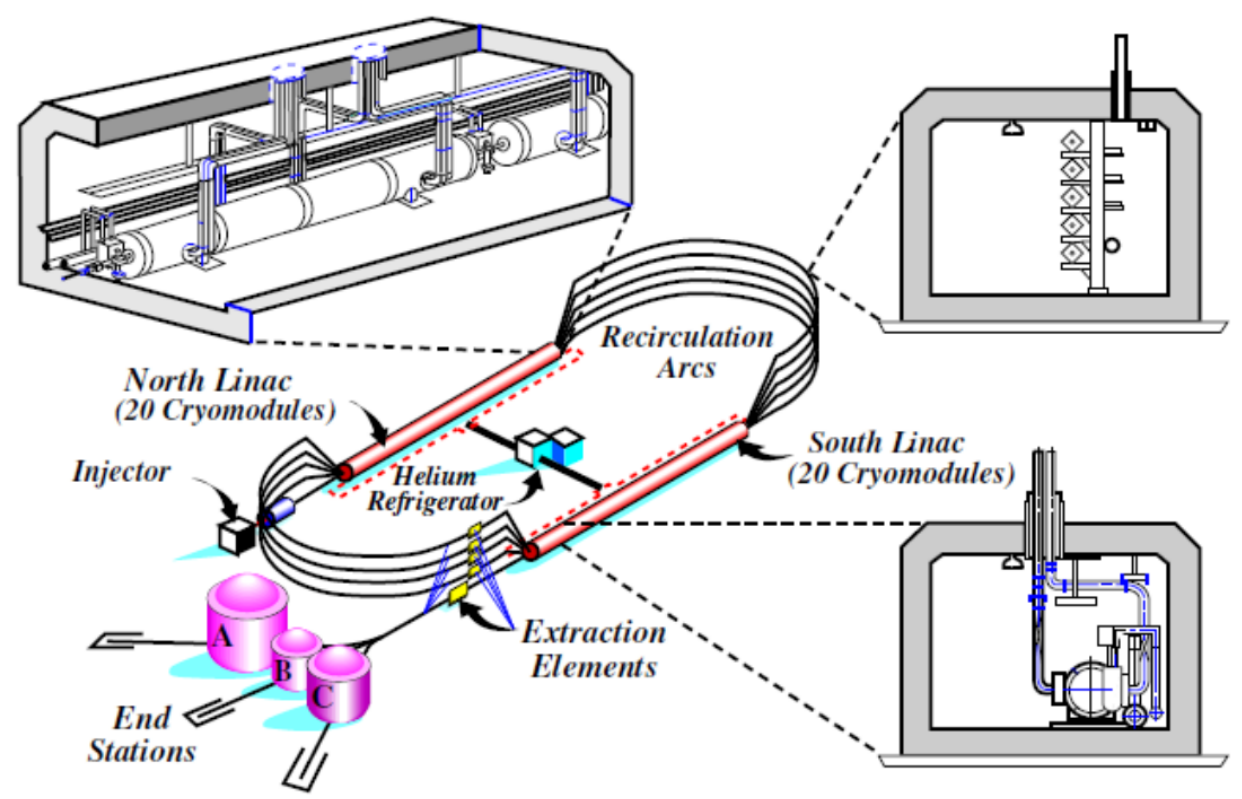

Figure 4.1: A schematic of CEBAF showing the injector, LINACs, and recirculation arcs.

The injector consists of three pulsed lasers, one for each experimental hall These are synchronised and combined to strike a photocathode at a frequency of $499 \mathrm{MHz}$. This results in $2 \mathrm{~ns}$ beam buckets being delivered similtaneously to each hall. There are three independent lasers so that each hall can choose the characterstics of the beam they want, the current, energy, and the polarization.

The injector introduces electrons at an energy of $67 \mathrm{MeV}$ to the North LINAC. It contains 168 Niobium radio frequency (RF) cavities and is cooled to $\sim 2 \mathrm{~K}$ using liquid Helium in order to make them superconducting. This means much less power is need and it can run continuously, $100 \%$ duty cycle. In the RF cavities direct microwaves in phase with the electron beam are introduced. This creates a negatively charged area behind the electrons, and a positively charged area in front of them therefore causing them to accelerate forwards. This process in the separate LINACs accelerates the electrons by $\sim 0.6 \mathrm{GeV}$ so each full pass of the circuit accelerates them by $\sim 1.2$ $\mathrm{GeV}$. The current is limit is 5 passes therefore the energy of the beam ranges from $\sim 1.2$ 
$\mathrm{GeV}$ to $\sim 6.0 \mathrm{GeV}$

The recirculation arcs are needed in order to bend the beam towards the next LINAC. Without these recirculation arcs many more kilometres of LINACs would be needed. Dipole magnets are arranged in an arc so as to bend the beam 180 degrees. As the electrons can vary in their energy, we need multiple arcs of varying strength in order to bend the beam for all the different possible energies of the electrons; as such there are 5 arcs in the west end of the accelerator and 4 at the east end. The beam is separated into the corresponding arc for its energy, bent by the arc, and then the different energy bands are recombined and sent to the next LINAC. Alternatively, at the east set of recirculation arcs the halls can extract the beam using an RF separator [86].

\subsection{Electron Beamline}

The electron beamline enters Hall B about $25 \mathrm{~m}$ upstream of the tagging spectrometer (see figure 4.4). The section contains final steering elements and beam diagnostics. First the position of the beam needs to be monitored. This is done by the Beam Position Monitors (BPM). There are three BPMs situated at different positions along the beamline, the first resides $36.0 \mathrm{~m}$ upstream of the target, the next $24.6 \mathrm{~m}$ upstream, and the last $8.2 \mathrm{~m}$ upstream. However, the last BPM is not used in photon beam experiments as it is situated downstream of the Tagger magnet.

The second check is confirming that the beam has a suitable beam profile. This is done using the harps. There are three of these too, the first $36.7 \mathrm{~m}$ upstream of the target, the next $22.1 \mathrm{~m}$ upstream, and the last $15.5 \mathrm{~m}$ upstream. They are made of thin (20 $\mu \mathrm{m}$ and $50 \mu \mathrm{m}$ tungsten and $100 \mu \mathrm{m}$ iron) crossed wire which are orientated perpendicular to the beamline and vertically and horizontally with respect to the beam line. They are set up so that the wires move across the beamline at 45 degrees from the horizontal axis. As it passes, the emitted Cherenkov light created by the scattering of the electrons is detected by photomultiplier tubes (PMTs). An example diagram of the resulting beam profile from this procedure can be seen in figure 4.2. As this is 
a destructive process it is only down when not taking data and is usually performed when new beam settings are being used or if there has been a problem with the accelerator [87].
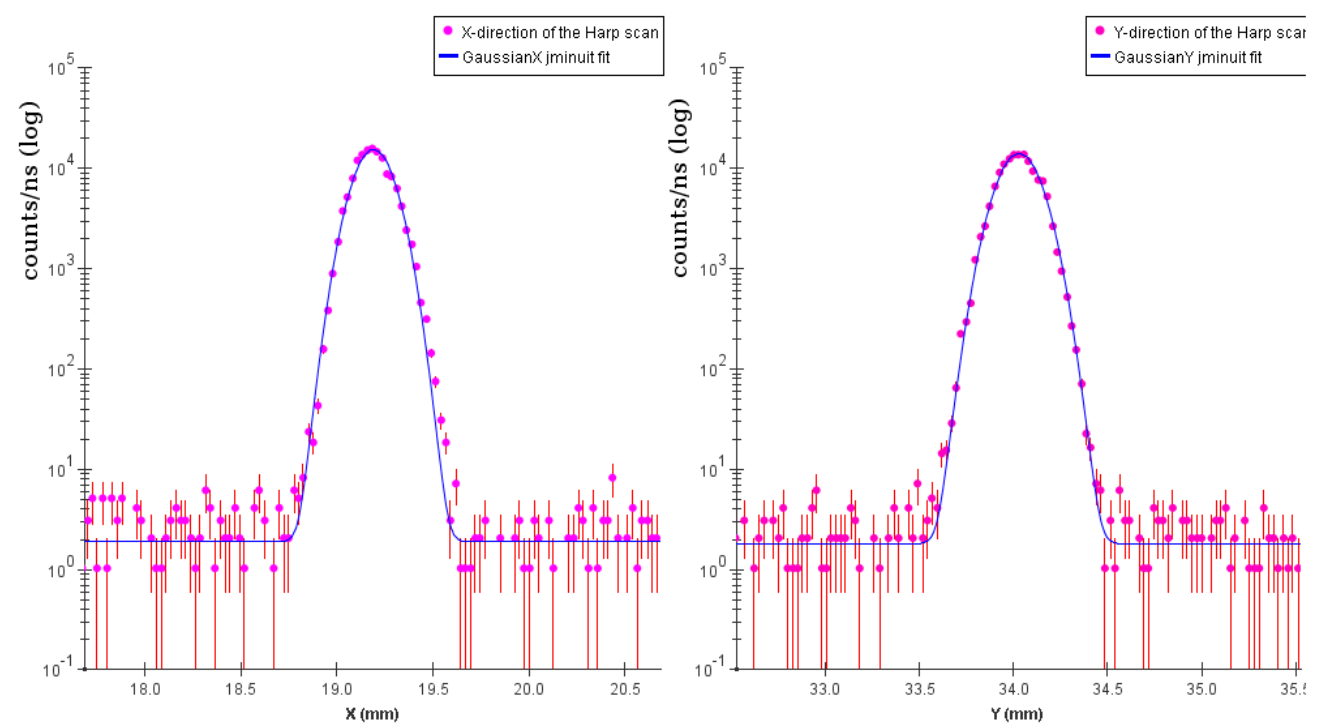

Figure 4.2: An example harp scan showing an ideal beam profile in both the $\mathrm{x}$ and $\mathrm{y}$ axes.

\subsection{The Tagging Spectrometer}

Hall B can used either an electron beam or a photon beam. This experiment was undertaken with a photon beam and so we need to use the tagging spectrometer which consists of a large dipole magnet and a focal plane hodoscope. The tagged photons are produced by the Bremmstrahlung radiation caused by the slowing of the electrons due to the electromagnetic field of the nuclei in a radiator. The tagged photons can have energies between $20-80 \%$ of the radiating electron beam energy. The magnet is a large c-shaped electromagnet which can produce a magnetic field with up to $1.75 \mathrm{~T}$. The size of the Tagger magnet needed for the energies being used at JLab means that the magnet is very large and has sagged in between the support beams. The calibration of this sag is described in [88]. The magnet bends all of the electrons sending those which have created a photon to the T- and E- counters and those which have not to the 
beam dump.

The E-counters are aligned so that they lie in the focal point of the Tagger magnet. There are 384 plastic scintillators of $20 \mathrm{~cm}$ length and $4 \mathrm{~mm}$ thick. Their widths vary so that each corresponds to the same momentum range and they vary from 6-18 $\mathrm{mm}$ width. They overlap so that each scintillator covers a third of the width of each of its neighbouring scintillators. So there are 767 e-channgels which gives us an energy resolution of $0.0013 \times$ the energy of the electron beam. The position of the detected electron measures the energy of the electron after Bremmstrahlung radiation $\left(E_{e}^{f}\right)$ and the initial energy $\left(E_{e}^{i}\right)$ is known as it iset by the accelerator. The energy ( $E_{\gamma}$ of the tagged photon is then simply calculated $\left(E_{\gamma}=E_{e}^{i}-E_{e}^{f}\right)$.

The T-counters give the timing of the photon. A timing resolution of at least 300 ps is needed in order to correlate a measured of an electron with the corresponding electron bunch. This then allows us to determine the timing of the hoton at the target. The resolution is $\sim 100$ ps which exceeds our needs. There are 61 scintillator counters, $2 \mathrm{~cm}$ thick, with varying lengths; $20 \mathrm{~cm}$ for the end of the array of scintillators associated with higher energy electrons, and $9 \mathrm{~cm}$ for those associated with lower energy electrons. A diagram of the tagging facility can be seen in figure 4.3 [89]. 


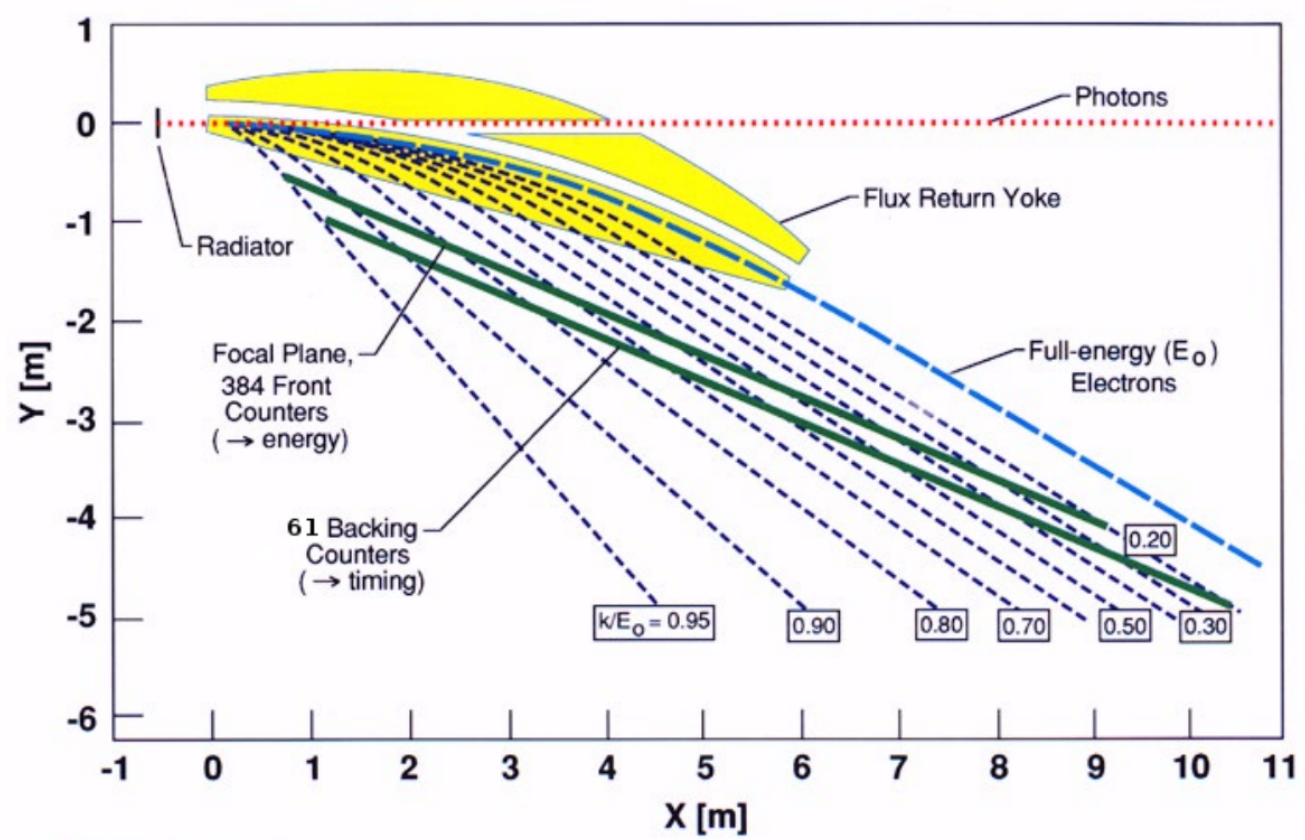

Figure 4.3: A diagram of the Tagger magnet, the T-counters, and E-counters showing their nature and their relative positions. The dashed lines are examples of potential electron paths after undergoing Bremmstrahlung radation [85].

\subsection{Coherent Bremsstrahlung}

Bremsstrahlung radiation occurs due to the slowing of the electron by the nuclei's electromagnetic field in a radiator. The created photon then radiates in the direction of the incident electron. Two different radiators were used in this experiment. The first was an amorphous radiator of carbon $50 \mu \mathrm{m}$ thick. The resulting photon spectra from this radiator exhibits a $\frac{1}{E_{\gamma}}$ behaviour. The other radiator was diamond which was also $50 \mu \mathrm{m}$ thick. The resulting spectra from this radiator has discrete peaks due to the coherent radiation effect these sit atop the same incoherent $\frac{1}{E_{\gamma}}$ background of the amorphous spectra. An example of a spectrum from both radiators is shown in figure 4.5. This is the reason why an amorphous radiator was used so as to remove this incoherent background. More detail of this process and Bremsstrahlung radiation as a whole is given in chapter 5 . The choice of the radiator is very important. A very thin radiator is needed so as to minimise electron scattering, which lowers the polarization 
of the photon beam, and the radiator also needs to have as few defects as possible for the same reason [90].

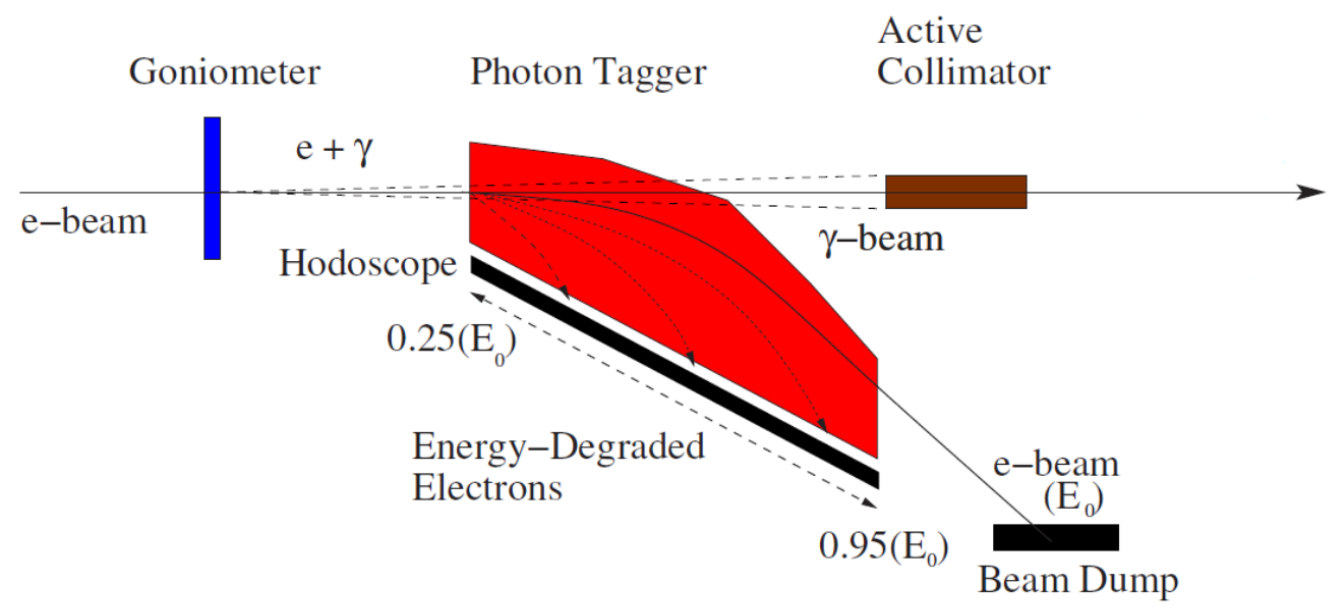

Figure 4.4: A schematic of the tagger facility and all of the associated systems [89].

In addition to the radiator characterstics, the electron beam variance and the angle of incidence with respect to the lattice structure of the diamond affects the polarization of the photon beam. The electron beam variance is minimised using the harp as described above. In order to control the angle of the radiator the Klein-Livingston Goniometer is used. This can set angles with a precision of $10 \mu \mathrm{rad}$ throught the axes. It also has a target ladder where the different radiators are housed so that the movement from one radiator to another is automated. The method use to find the optimum angle is described in more detail in [91]. 


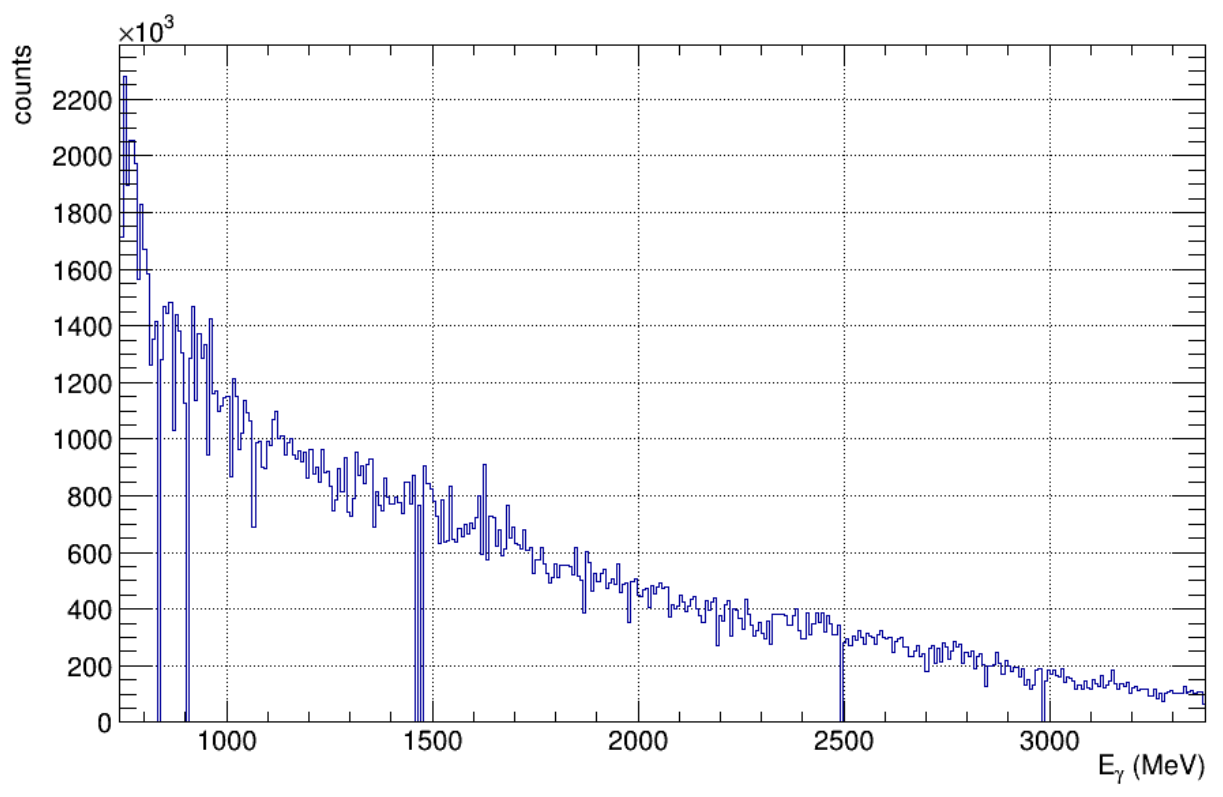

(a)

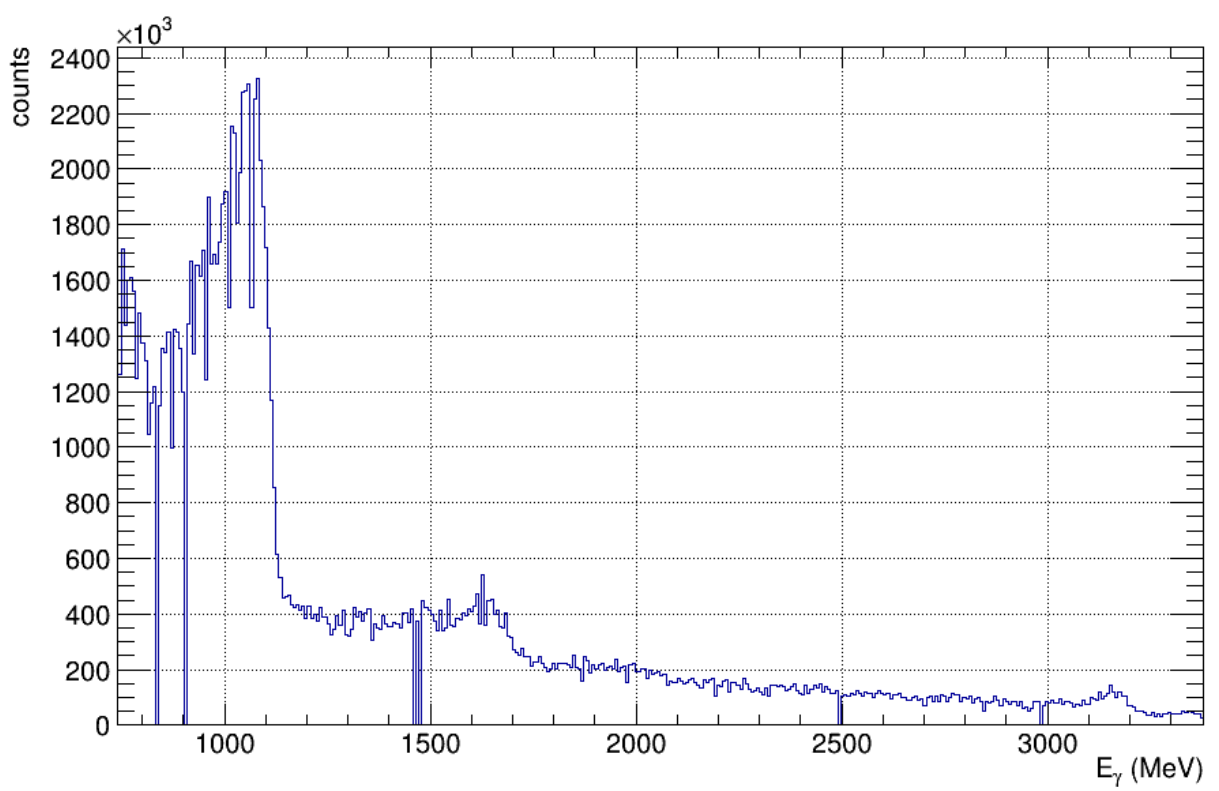

(b)

Figure 4.5: Typical photon spectrum using the amorphous (a) and diamond (b) radiators.

\subsubsection{Active Collimator}

A collimator improves the polarization of the beam as the coherent component is more forward focused than the incoherent. The collimator used in this experiment is active; 
there are $4 \mathrm{~mm}$ plastic scintillators between each layer to measure pair production so as to have a real-time monitor of the beam's position. The collimator is situated 22.9 $\mathrm{m}$ downstreamof the goniometer. It is made of 13 nickel diskettes with a diameter of $50 \mathrm{~mm}$ and a thickness of $15 \mathrm{~mm}$ with a $2 \mathrm{~mm}$ hole in the centre [92].

\subsection{Target}

The target housing is located $20 \mathrm{~m}$ downstream of the tagger. The target used in this experiment was placed in a $40 \mathrm{~cm}$ long, $2 \mathrm{~cm}$ radius Kapton cylinder cell. It was positioned so that the centre was $20 \mathrm{~cm}$ upstream of the centre of CLAS. The target itself was liquid Hydrogen kept at a density of $0.071 \mathrm{~g} / \mathrm{cm}^{2}$ [85].

\subsection{The CEBAF Large Acceptance Detector}

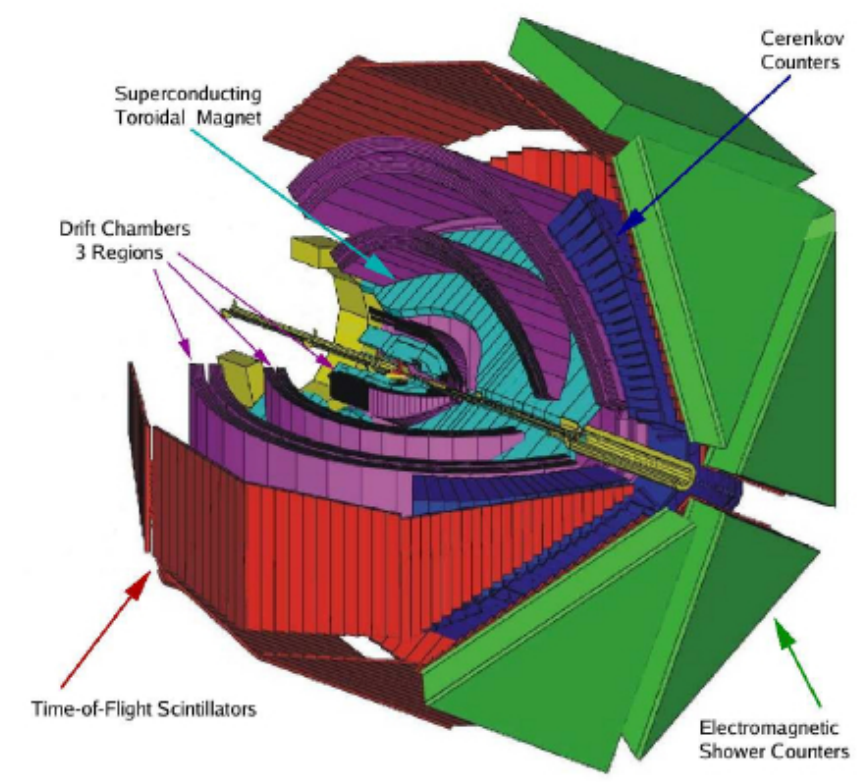

Figure 4.6: A 3D diagram of the CLAS detector showing the subsystems [87]. 
The CEBAF Large Acceptance Spectrometer (CLAS) is a detector made up of many sub-systems which has an almost $4 \pi$ coverage around the target. It is capable of measuring charged and uncharged particles, with a prediliction for charged particles. It is built around a toriodal magnet which bends charged particles away or towards the beamline depending on whether the particle is negative or positive. The shape of the magnet also means that in the target there is no field. This means that highly polarized targets can be used in the CLAS detector. Precise measurements of the characterstics of the produced particles, such as timing, momentum, charge, and mass, come from the many sub-systems. These will described in this section [87]. The detector as a whole can be seen in figure 4.6 and figure 4.7 .

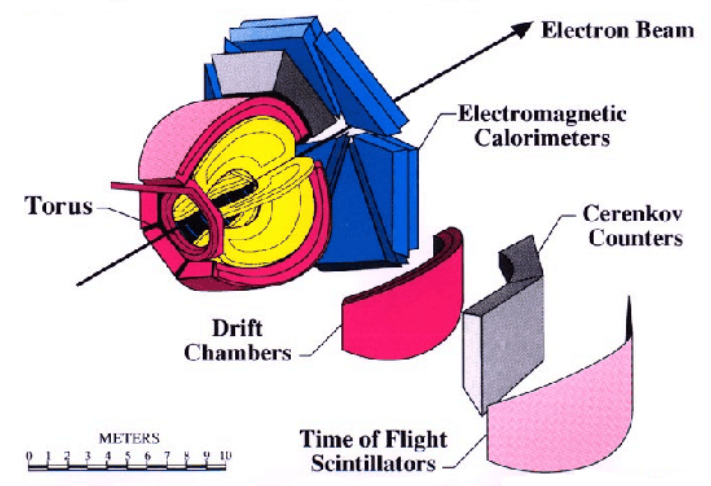

(a)

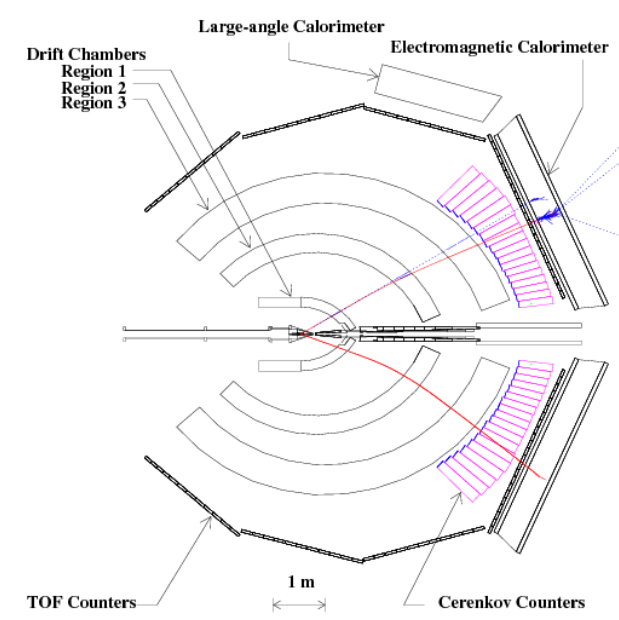

(b)

Figure 4.7: Shown in (a) is an exploded diagram of a CLAS section. Shown in (b) is a cross section of the detector as seen from above with two simulated tracks of charged particles (red lines) with the top track being positive and the bottom negative [87].

\subsubsection{Torus Magnet}

The CLAS detector was desgined around the use of a toroidal magnet, the Torus magnet. It gives good momentum resolution for charged particles while also having a large angle coverage. In addition to this, there is a field-free area in the centre for the target so polarized targets can be used. 
The magnetic field comes from siz superconducting coils arranged to form a toriodal shape around the beamline. The field points in the $\phi_{L A B}$ direction and divides the detector into six sectors. The Torus magnet is $5 \mathrm{~m}$ in diameter and $5 \mathrm{~m}$ long. The superconducting coils are kept at $\sim 4.5 \mathrm{~K}$ by pushing supercritical Helium through cooling tubes at the end of the windings of the coils. Each coil contains four layers with each layer having 54 turns of wire made of $\mathrm{NbTi} / \mathrm{Cu}$ stablised by aluminium. The field strength varies to account for the differences in momentum for particles in different areas of the detector; the field is $\sim 2.5 \mathrm{~T}$ in the forward angle and $\sim 0.5 \mathrm{~T}$ perpendicular to the beamline [93].

\subsubsection{Start Counter}

The first detector system that the produced particles meet is the Start Counteras it surrounds the $40 \mathrm{~cm}$ target cell giving complete coverage. It gives a precise start time for every triggered event and the time the photon was at the interaction vertex when coupled with information from the Time of Flight (ToF) system. It has six sectors which are further broken down into four paddles. Each paddle has a straight section of $502 \mathrm{~mm}$ and a bended end so that it tapers to a point in the downstram direction. The other end is connected to a light guide and a PMT. The timing resolution is $290 \mathrm{ps}$ and 320 ps for the straight and bent component, respectively; this level of resolutions necessary in order to accurately determine the velocity of the particles [94]. 


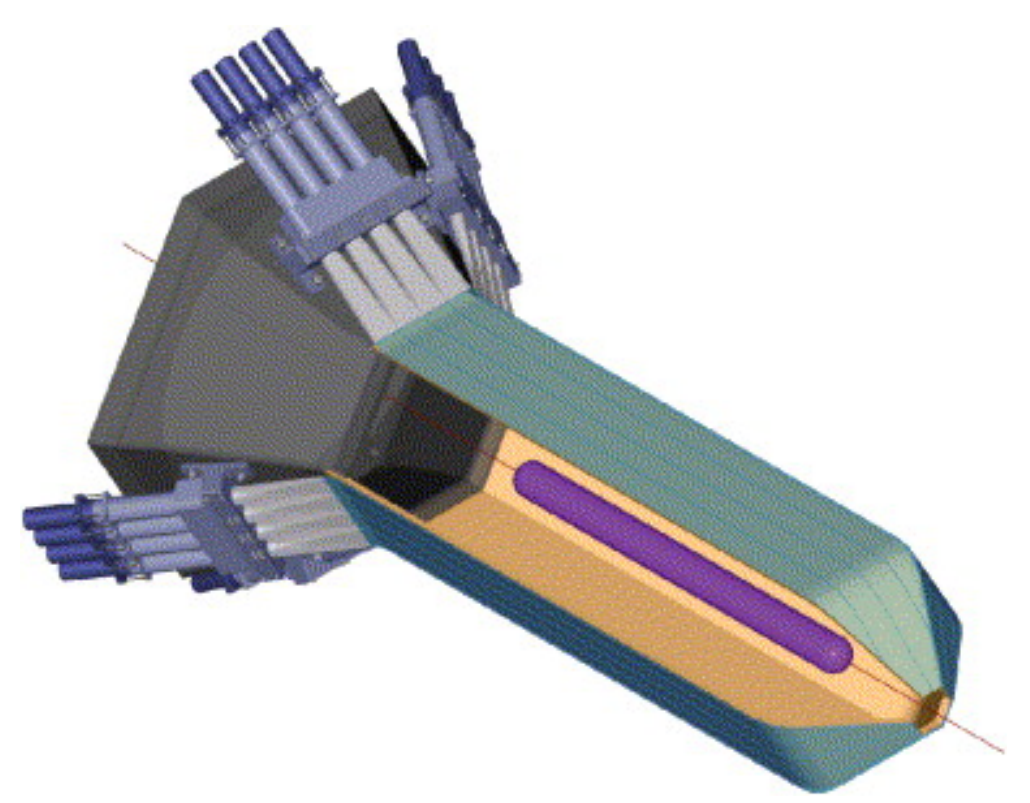

Figure 4.8: A 3D diagram of the Start Counter [95].

\subsubsection{Drift Chambers}

The next layers of the dector harbour the CLAS Drift Chambers. These are used to track particles so as to discern particle type and its momentum. The system consists of 18 separate drift chambers divided into the 6 sectors of the detector and into 3 layers. The three layers are referred to as region 1, region 2, and region 3. Region 1 is closest to the target, residing inside the torus coil in an area of very low magnetic field. It is predominately used to determine the starting point of a particle trajectory. Region 2 is located between the coils so is in an area with a very high magnetic field. Therefore, the paths of the particles will be strongly altered in this region. As the maximum curvature of the particle occurs here, region 2 has the best momentum resolution. Region 3 is furthest away from the target and again is in a low magnetic field area. It is used for determining the end point of the particle's path. The angular coverage of the Drift Chambers system is almost complete; 8 - 142 degrees for the polar angle, complete coverage for the azimuthal angle except for the fiducial regions in between the 6 sectors resulting in $\sim 80 \%$ coverage [96]. 


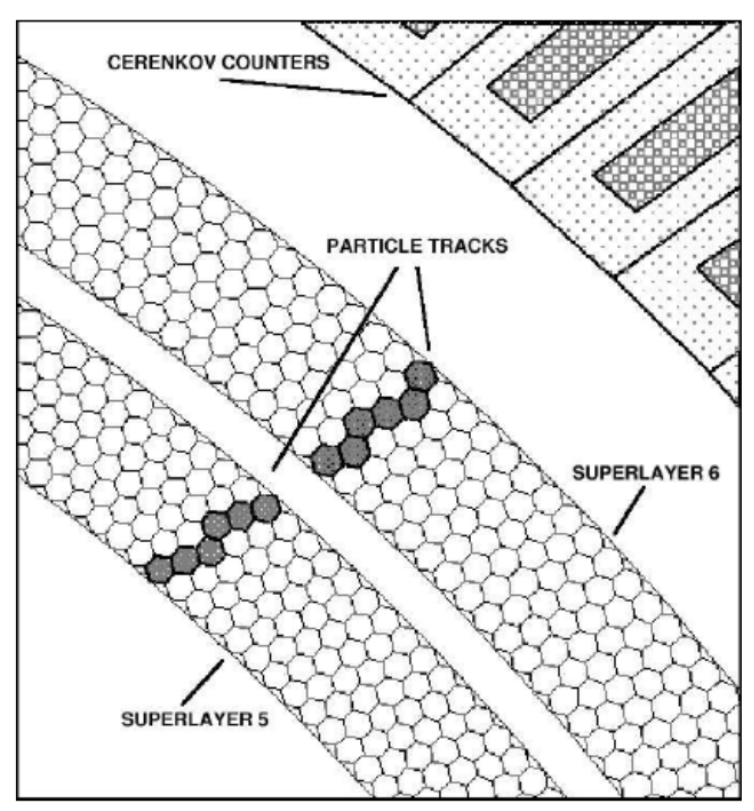

Figure 4.9: A diagram showing the layers and cells of the CLAS Drift Chambers. The highlighted cells shows a simulated path of a particle through the superlayers of the drift chambers [97].

Each region contains two superlayers with each having six layers of wires. The first superlayer is positioned axial to the magnetic field and the second is positioned stereo to the magnetic field. The stereo layers are positioned 6 degrees stereo to the magnetic field in order to provide azimuthal information. Surrounding each super layer is a layer of guard wires with very high-voltage potentials in order to reproduce an electric-field configuration similar to an infinite number of cells; this means that the electric field at each sense wire is the same for all of them rather than varying depending on the layer number.

Each drift chamber is arranged with its sides parallel to the neighbouring Torus coils and are position 60 degrees with respect to each other. This means that the wires in the superlayers are perpendicular to the path of the particles which gives the maximum sensitivity possible. Each chamber is filled with $88 \%$ argon and $12 \%$ carbon dioxide. The layers of wires in each superlayer were arranged so that they were offset by half the width of the cells in order to increase packing in the volume available. The cells are formed hexagonally with field wires positioned at the vertices of the hexagon cell and a sense wire in the centre. The diagram in figure 4.9 shows these superlayers 
and the cells for one drift chamber region. The sense wires are gold-plated tungsten with a radius of $20 \mu \mathrm{m}$. The field wires are gold-plated aliminium with a radius of 140 $\mu \mathrm{m}$. Hexagonal cells were chosen because in such cells the drift time and drift distance are independent of a particles incident angle upon entry into the cell [97].

\subsubsection{Time of Flight Scintillators}

The ToF scintillators are situated about $4 \mathrm{~m}$ from the target between the Cerenkov counters and the electromagnetic calorimeter; these two detectors are used for electron beam experiments where negative pion and electrons need to be separated. For more information on the Cerenkov counters see [98] and for the electromagnetic calorimeter see [99]. The ToF system consists of 6 panels, one for each sector. It has the same polar coverage as the CLAS Drift Chambers and complete azimuthal coverage. Each panel contains 57 scintillator paddles orientated perpendicular to the average particle trajectory in each paddles region. Each paddle is $5.08 \mathrm{~m}$ thick and made of Bicron BC-408 scintillator with a PMT attached at each of the paddle. The output from the PMTs are sent to ADCs and go throud discriminator to TDCs which record the size and timings of the pulses. The paddles vary in length from $32 \mathrm{~cm}$ to $445 \mathrm{~cm}$ depending on its location in the panel. The width of the paddle is dependent on its placement with regards to the polar angle; in the forward region $(\theta<45) 15 \mathrm{~cm}$ and in the backward region $22 \mathrm{~cm}$ [100].

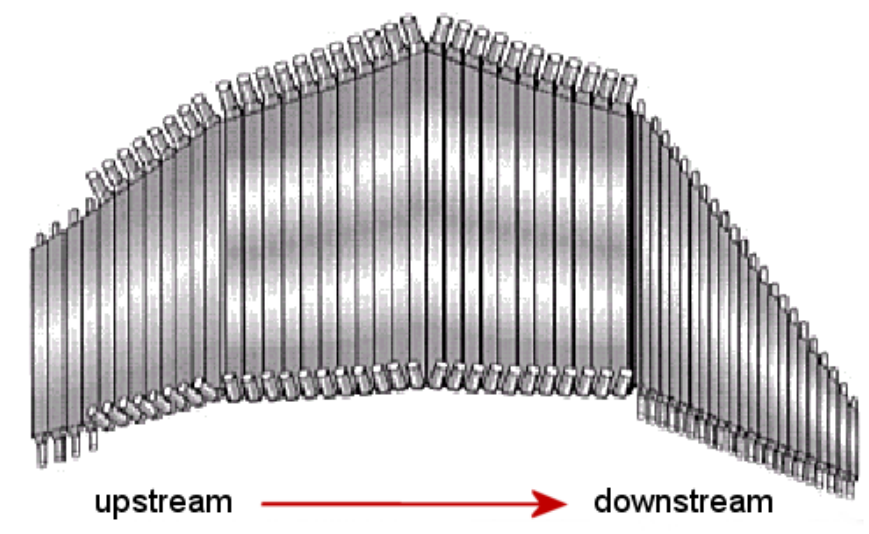

Figure 4.10: One section of the ToF system [100]. 
The timing info from the ToF systems and the Start Counter allow for the $\beta$ of particles to measured ( $\beta=$ fracvc. Used in conjunction with momentum measurement from the CLAS Drift Chambers and the charge of the particles, rough estimations of particles'mass can be made and the particles can be identified. Also, the ToF system was used in the g8b experiment as the CLAS Level 1 Trigger. The ToF was used instead of the Tagger because the latter had too high a count rate for the trigger.

\subsection{Trigger System}

Each detector sub-system is attached to electrons, but in order for data to be collected triggers are used. A trigger is a condition or a set of conditions which must be met in order for data to be recorded by the Data Acquisition System (DAQ). These conditions are chosen in order to prevent background processes such as cosmic rays or electronic noise from the diluting the data. They can also be set so as to restrict the final state particles if an experiment has a well defined, single process of interest. This was not the case for $\mathrm{g} 8 \mathrm{~b}$ and the trigger system used only had restrictions to remove background processes. Two triggers were used; the Level 1 Trigger and the Level 2 Trigger.

Level 1 is the faster of the two triggers. It requires a hit in the Start Counter and a ToF scintillator in the same sector. The second trigger, which causes deadtime in the system, requires a reconstructable path in the CLAS Drift Chambers system. The first trigger accounts for potential electronic noise, the second for processes like cosmic rays. The Trigger Supervisor decides if an event will recorded or not. The supervisor can be set to require only the Level 1 Trigger be satisfied or to require both; for $\mathrm{g} 8 \mathrm{~b}$ both were required [101].

\subsection{Data Acquisition System}

The data acquired by the CLAS detector is recorded by the DAQ system. The data is digitised into 24FASTBUS and VME crates, which are situated in the experimental 
hall. The datastream is then collected by the 24 VME Readout Controller in the crates. If the Trigger Supervisor is happy, the data is converted into BOS format. The data is separated into sections which are referred to as banks. These banks contain the information from each of the detector sub-systems along with a unique event number associated with a given run number. The data is then transferred to a RAID array and final to a tape silo where it remains until needed for analysis [102].

The typical event rate for the g8b experiment was $\sim 4.5 \mathrm{kHz}$ which resulted in a dead-time of $\sim 13 \%$. The event rate was not restricted by the DAQ nor the trigger system, but by the Tagger. A low beam current of $\sim 10 \mathrm{nA}$ was used otherwise the number of photons produced would be too high and would result in part of the Tagger system not functioning efficiently [85].

\subsection{Summary}

The experiment was carried out using the CLAS detector and the coherent Bremsstrahlung facility of Hall B, JLab. Descriptions of these facilities and their sub-systems have been presented in this chapter along with details of the setup of these facilities.

At the final stage of the process described above, the data needs to be calibrated and formatted so it can be analysed. The process of calibrating the data for all the different sub-systems is called 'cooking' and data which is fully calibrated ready for analysis is called 'cooked data'. The following sections describes in detail how and why one system is calibrated, the tagged coherent Bremmstrahlung. Then the subsequent chapter begins dicussion of the process of analysis using the fully cooked data. 


\section{Chapter 5}

\section{Photon Polarization}

This section describes the theory of generating polarized photon beams using coherent Bremsstrahlung radiation and also the the work done on calculating the polarization of the photon beam used during the $\mathrm{g} 9 \mathrm{a}$ and $\mathrm{g} 9 \mathrm{~b}$ experiment. The same process was undertaken by another collaborator for the dataset used for the main analysis of this thesis, but the result of this process will be shown in general terms for this dataset.

\subsection{Theory}

A photon beam can either be circularly polarized or linearly polarized. In this experiment we used linearly polarized beams. This allows us to measure the sensitivity of the intermediate resonant states to the orientation of the electromagnetic field components of the exciting photon beam; this is the case as a linearly polarized photon beam has, by definition. its electric field vector (and conversely its magnetic field vector) restricted to a single plane with respect to the beam's direction of propagation. As described above, the beam asymmetry gives us a measurement of this sensitivity of the resonances by comparing the cross sections of photoproduced mesons using two different polarized photon beam orientations, one parallel to the $x-y$ plane and the other perpendicular to it. The beam asymmetry can be more sensitive in differentiating between different models than just measuring cross section and has the extra benefit of 
not requiring a detailed detector acceptance [103].

Bremmstrahlung radiation is caused by the slowing of a fast electron in an atomic structure due to the electromagnetic field of the nucleus. The lost energy of the electron then manifests as a photon [104]. The process is described as below:

$$
E_{i}=E_{f}+E_{k}
$$

Where:

$E_{i}, E_{f}$, and $E_{k}$ are, in order, the electron's initial energy, the electron's final energy, and the photon's energy.

The process is restricted by the longitudinal and transverse momentum of the recoil nucleus. Where $\delta(x)$ is the minimum recoil momentum in order to produce $\mathbf{x}$, $\mathbf{x}$ being $E_{k} / E_{i}$, the approximate restrictions are:

$$
\begin{aligned}
& 0 \leq q_{\text {trans }} \leq 2 x \\
& \delta \leq q_{\text {long }} \leq 2 \delta
\end{aligned}
$$

Only when a nuclei resides in this region of momentum space does it absorb the recoil momentum. If a diamond is being used, the placement of nuclei will be tightly regimented and momentum space will be occupied at discrete intervals. As the photon energy increases, or decreases, the region in momentum space where the process is restricted to will change so certain reciprocal lattice vectors will change from residing in the required region of momentum space to being outside of the region. This gives rise to a discontinuous energy spectrum. An ehancment plot is the resulting spectrum after subtracting a weighted coherent spectrum from from a coherent one. An example of such a spectrum is shown in figure 5.1 [105]. 


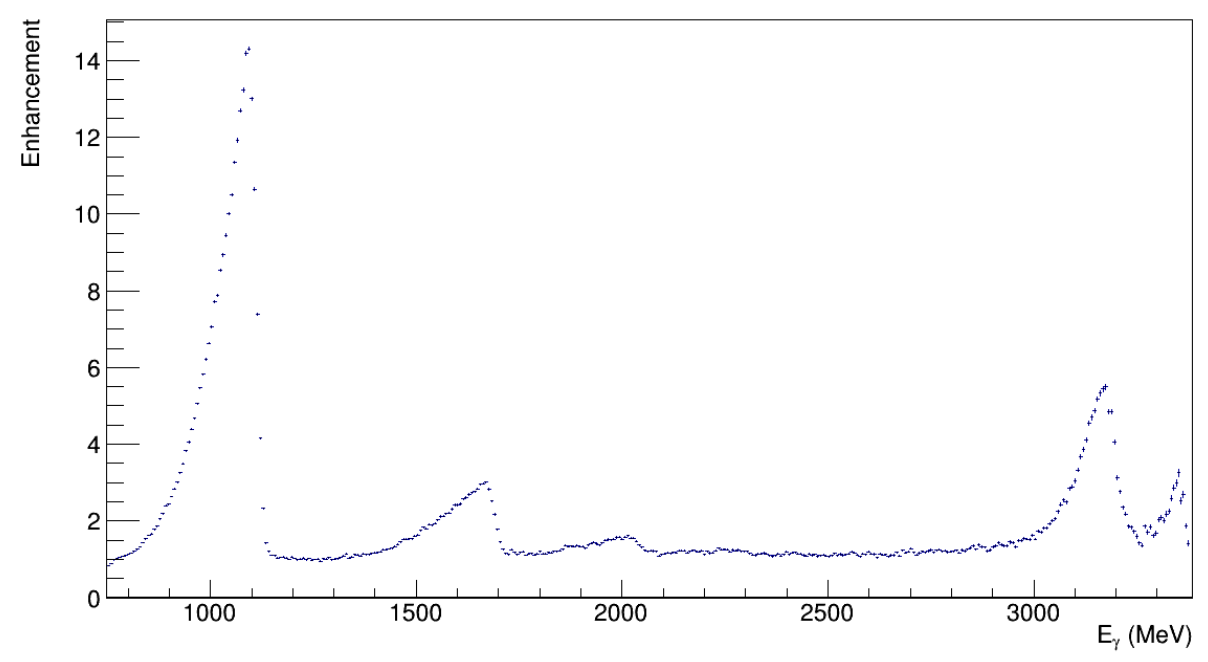

Figure 5.1: A typical enhancment plot for the measured coherent edge position of $1.109 \mathrm{GeV}$ for the $1.1 \mathrm{GeV}$ dataset. An enhancement plot is the spectrum achieved by subtracting a weighted incoherent spectrum from a coherent spectrum.

It was shown by Überall that these discrete regions of the spectra from crystals have a very strong linear polarization [106][107]. The interference effects which cause these high level of polarization are described by May [108]. These result in regions of high polarization, which are referred to as Coherent Peaks, and their position in the photon energy spectrum is set by adjusting the small angle between the crystal lattice and electron beam.

The relationship between the electron beam orientation with respect to the lattice structure and the resulting photon energy, flux, and polarization will be described in more detail below when discussing the fitting procedure of the $E_{\gamma}$ spectra.

The strongest degree of polarization is obtained by scattering from the 022 (and 00-2) lattice vector. This is illustrated in figure 5.2 where the incident beam is alligned with the 100 direction. The angle between the 022 direction is changed in order to manipulate the position of the discontiuties in the photon spectrum. The angle $\phi$ determines the azimuthal orientation of the polarization plane. The angle $i$ is set at a value $\sim 5$ times that of $\theta$ in order to ensure that no other coherent contributions from orthogonal lattice points convolute the coherent peaks. Also, a very thin crystal is 
optimal as this lowers the incidents of the electrons undergoing multiple scattering which lowers the polarization of the resulting photon beam by spreading the range of polar angles, $\theta$, which subsequently spreads the width of the peaks of the spectra and it also introduces a systematic uncertainty in the tagging process. Furthermore, an electron beam with a very low divergence is needed so as to also minimise the spread across $\theta[105]$.

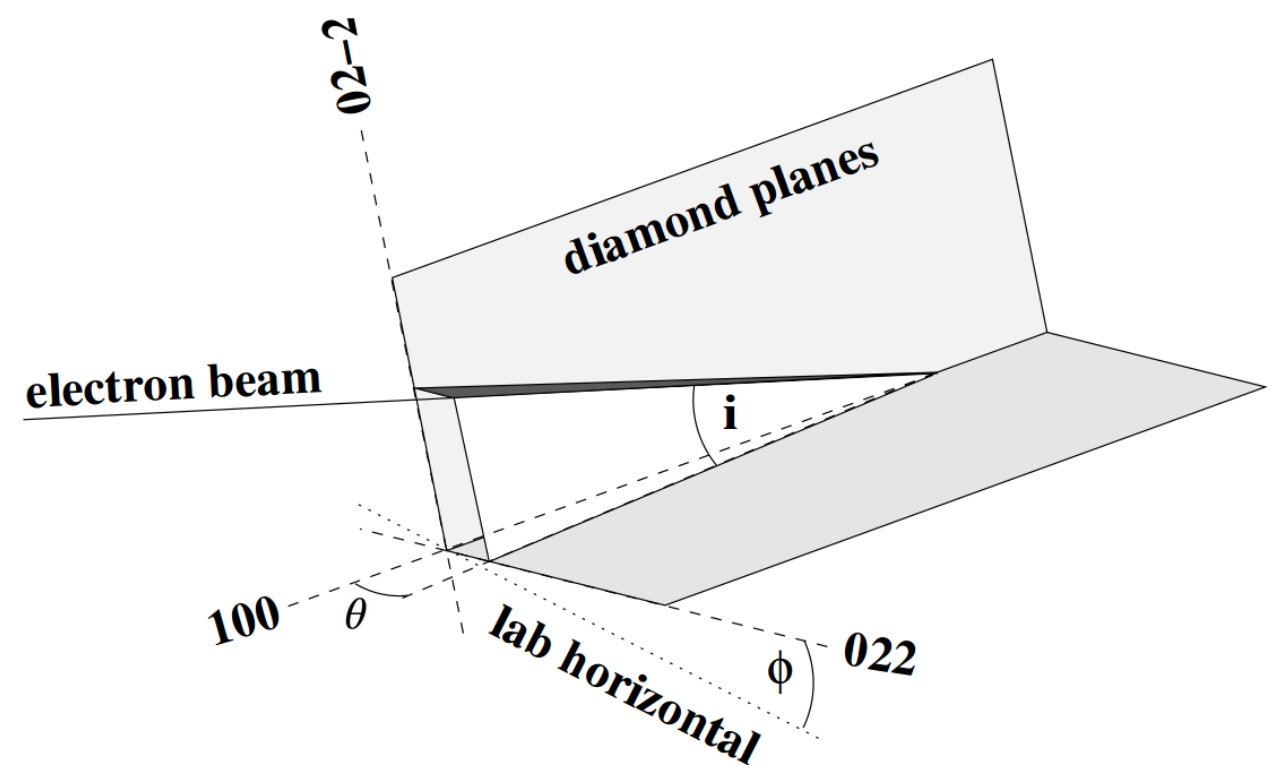

Figure 5.2: The angles involved for coherent bremsstrahlung from a diamond in the 100 orientation. The angle $\theta$ is the angle which is manipulated so as to attain a coherent edge of the desired energy.

\subsection{Enhancement Spectra}

The first step is to get the corrected photon energy spectra for the coherent and amorphous data. This is done by looking at the timing of the photons. The timing used is taken from the tagger hits and a cut of $\pm 30 \mathrm{~ns}$ around $0 \mathrm{~ns}$ is applied, the assumption that anything with a timing difference greater than 30ns are random photons. An example of the separation of the prompt photons and the random photons is shown in figure 5.3. The weighted random spectra are subtracted from the prompt spectra to give us corrected spectra for the coherent and incoherent data. 


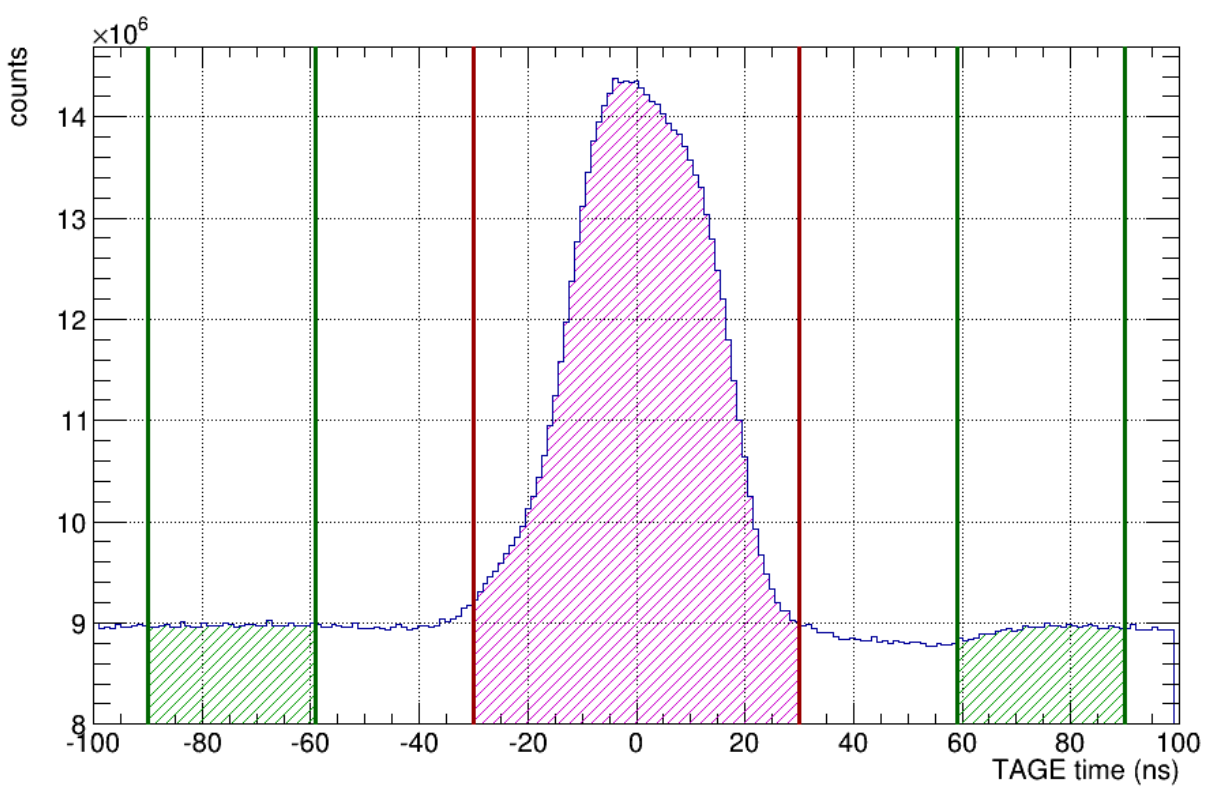

Figure 5.3: A typical timing plot using the tagger information after calibration. The prompt region is highlighted in magenta and the two random regions are highlighted in green.

The prompt, random, and corrected energy spectra for amorphous data for the 1.1 $\mathrm{GeV}$ run from the g9a dataset can be seen in figure 5.4,5.5, and 5.6, respectively. Similarly, 5.7,5.8, and 5.9, show the corresponding spectra for the parallel data from the $1.1 \mathrm{GeV}$ data.

The next step is to obtain enhancement spectra. A corrected spectrum from a polarized run with a diamond radiator is divided by a corrected energy spectrum from a run with an amorphous radiator. The baseline is then normalised to have an intensity of $\sim 1$. This process removes the $\frac{1}{E_{\gamma}}$ dependency of the polarized energy spectrum. Amorphous runs were undertaken throughout the data collection. This is so that small channel-to-channel variations due to counter problems in the tagger and any dead counters are accounted for. 


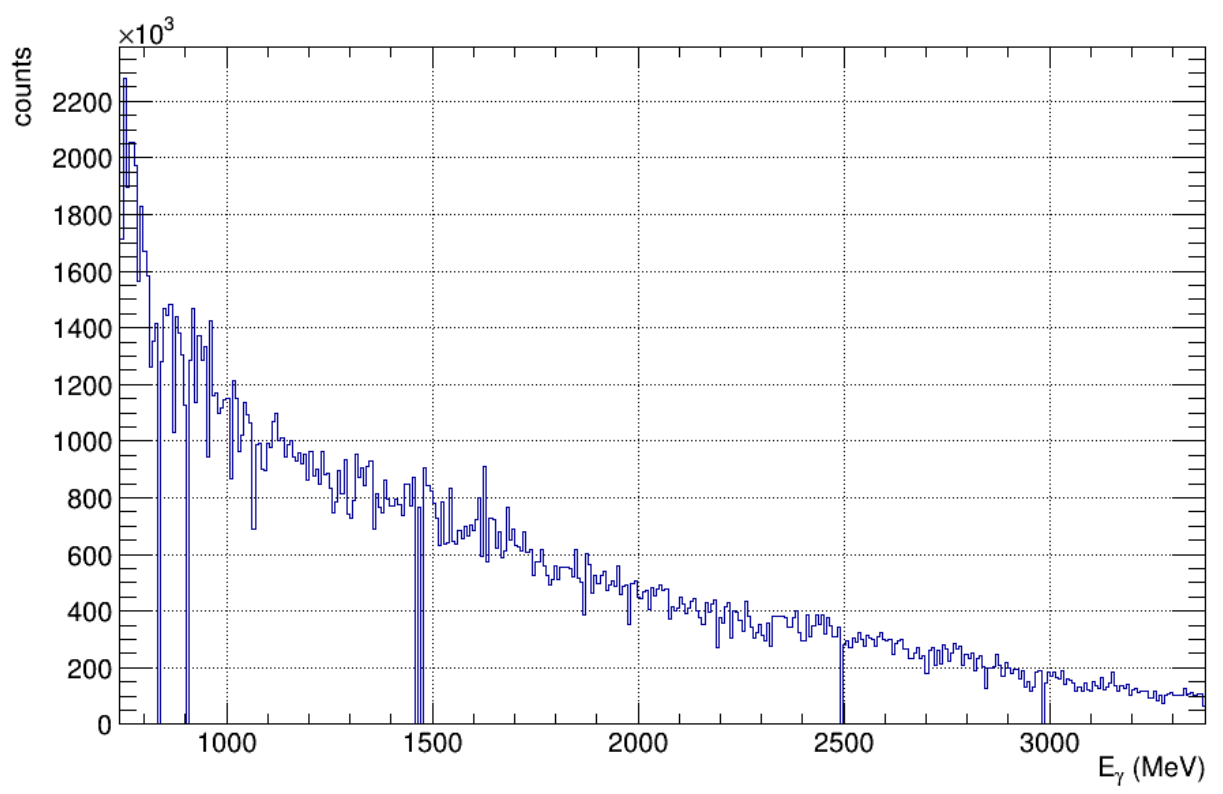

Figure 5.4: The the amorphous energy spectrum for the $1.1 \mathrm{GeV}$ data for the prompt region.

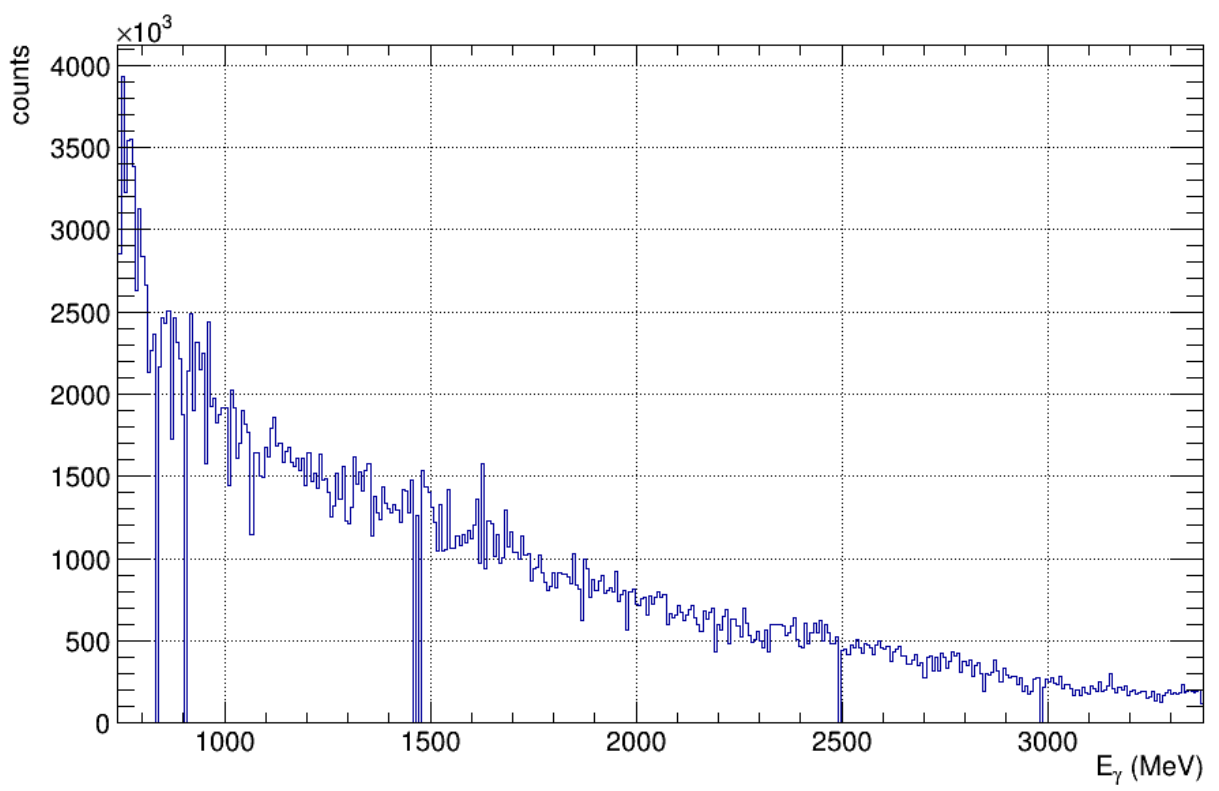

Figure 5.5: The amorphous energy spectrum for the $1.1 \mathrm{GeV}$ data for the random region. 


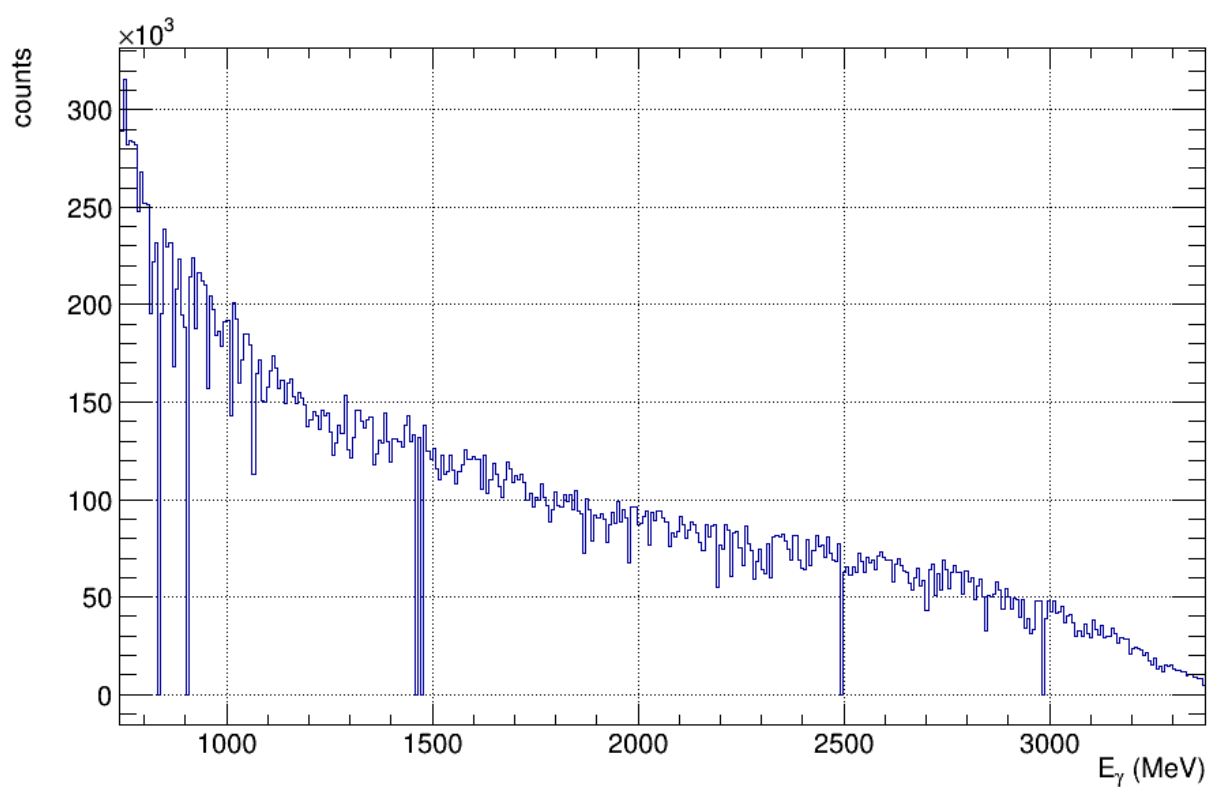

Figure 5.6: The corrected amorphous energy spectrum for the $1.1 \mathrm{GeV}$ data.

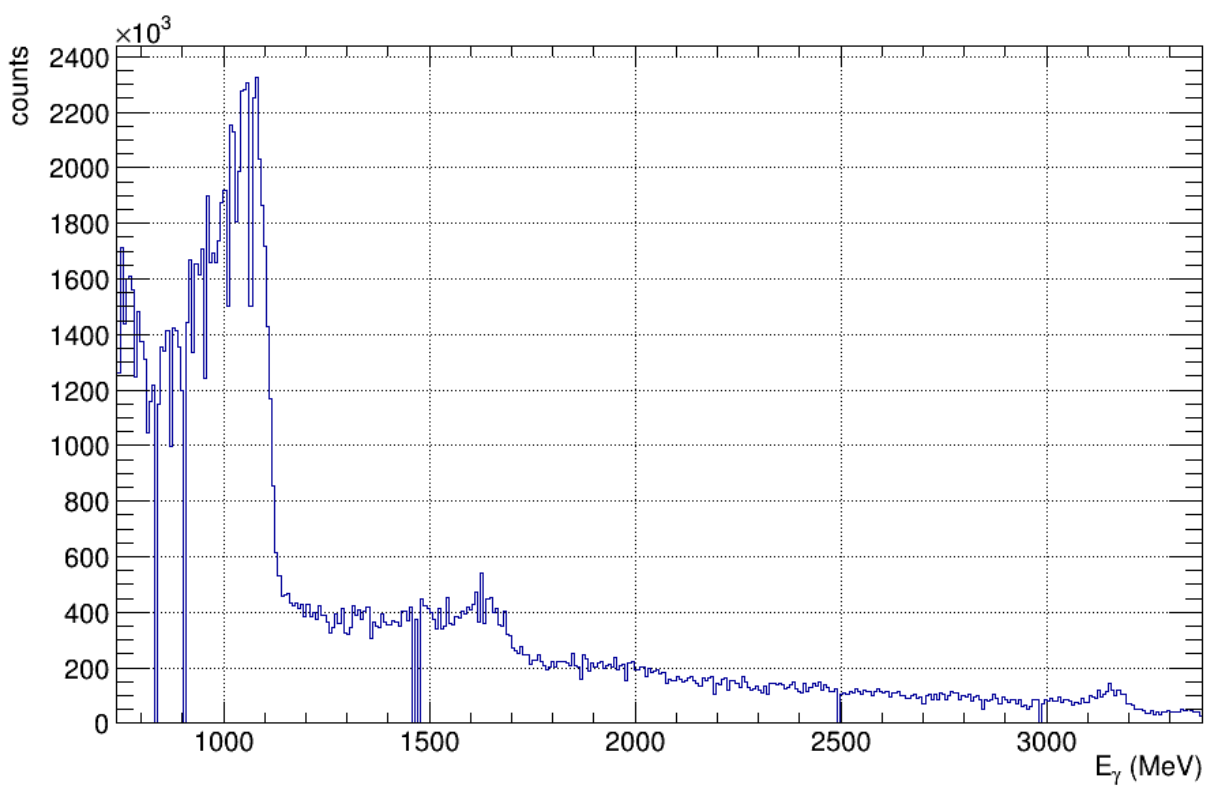

Figure 5.7: The the parallel polarization energy spectrum for the $1.1 \mathrm{GeV}$ data for the prompt region. 


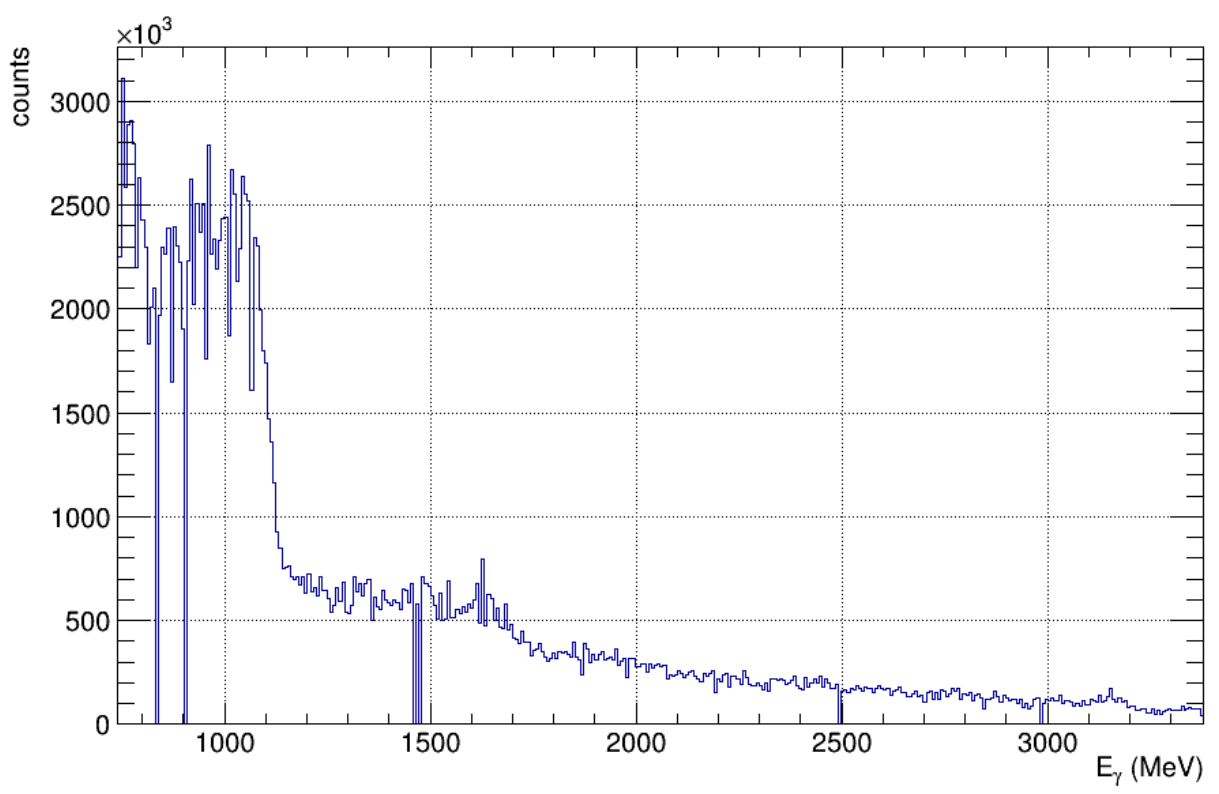

Figure 5.8: The parallel polarization energy spectrum for the $1.1 \mathrm{GeV}$ data for the random region.

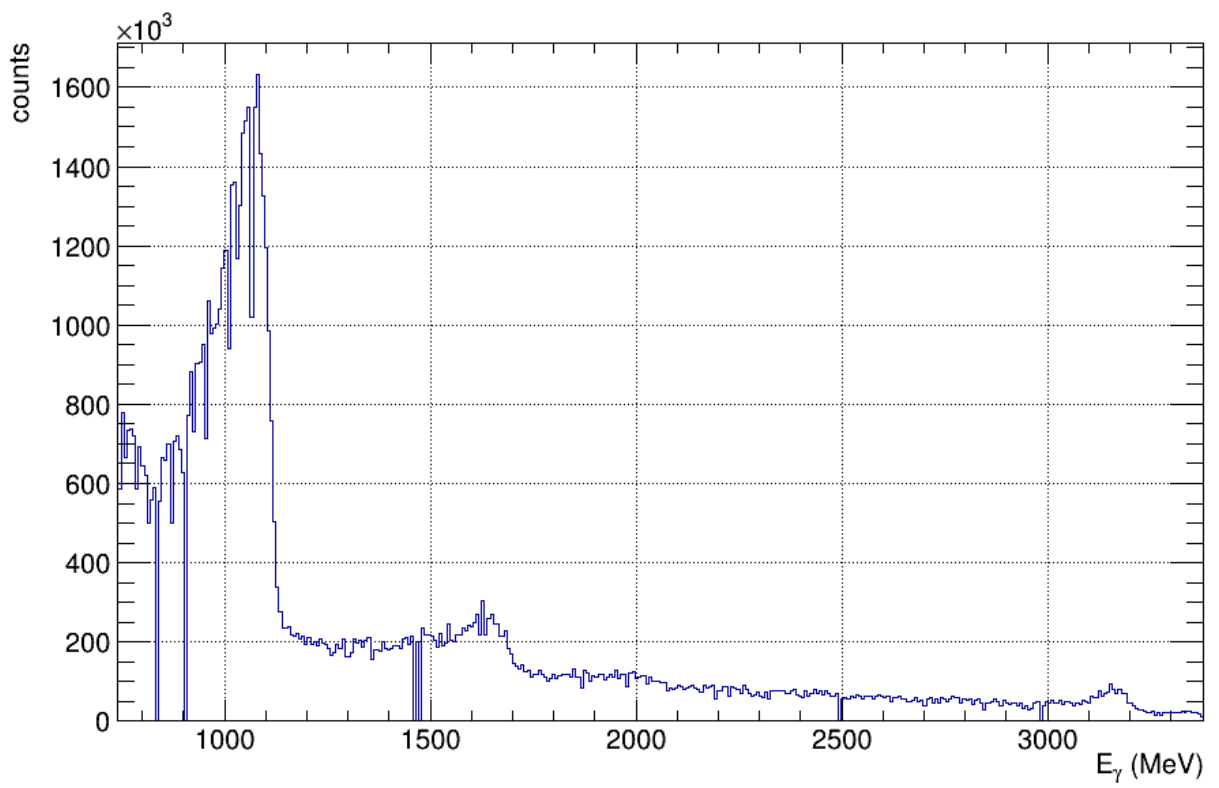

Figure 5.9: The corrected parallel polarization energy spectrum for the $1.1 \mathrm{GeV}$ data.

Enhancment spectra are obtained for each TDC settings, so the measured coherent is plotted against the corrected energy spectrum as shown in figure 5.11 and for each $2 \mathrm{MeV}$ bin of the measured coherent edge position is projected and divided by the 
amorphous data to attain individual enhancement plots. An example of this for the coherent edge position $1.109 \mathrm{GeV}$ is shown in figure 5.1.

\subsection{Moving Diamonds}

The coherent edge is set using the methods described above in section XX for different energies. However, during the experiment the coherent edge moves due to small movements of the radiator. This effect can be seen in figure 5.10. Therefore the actual coherent edge is different from the intended edge position by a significant amount. This affects the polarization of the beam and has to be calibrated.

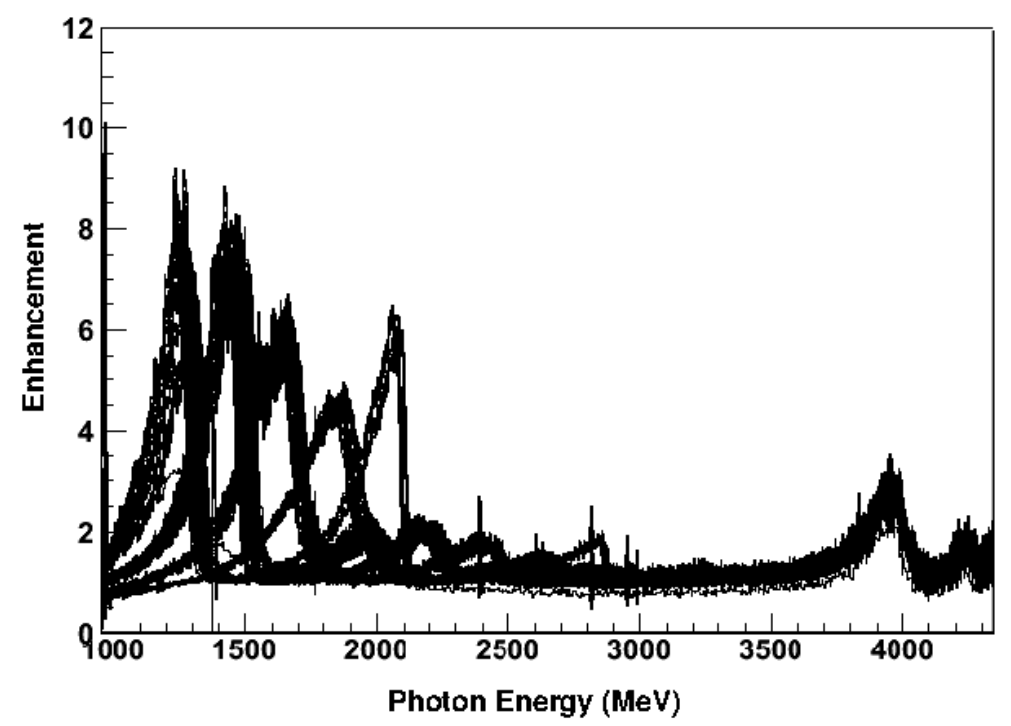

Figure 5.10: The shift for the coherent edge position for each beam setting for the $\mathrm{g} 8 \mathrm{~b}$ data.

Real-time enhancement plots, which are spectra showing the ratio of coherent and incoherent Bremsstrahlung radiation scaled so that the baseline is $\sim 1$, are constructed from the current uncollimated scaler counters and the most recent scaler readout from a run with an amorphous radiator rather than from the tagger information. This is because it takes about five minutes to build a suitable enhancement using the DAQ. We use these real-time enhancement plots to monitor the edge position in case it moves too far from the desired edge position and to check that beam is actually polarized. 
Therefore, the calibration is done off-line after the data is collected.

\subsection{Deriving a Fit}

The first peak of the enhancement spectrum corresponds to the 022 lattice vector and it is this area that we are interested in; it has the highest enhancement of the spectrum and therefore the highest polarization and is the vector used for alligning the electron beam with the diamond [105]. There are two contribution to this area of the spectrum; the coherent contribution from the 022 vector and the incoherent Bremsstrahlung contribution. The latter is more or less removed by the above process but the fit derivation below will be followed presuming there is still a meaningful contribution under the peak [109]. Also, the peak corresponding to the radiation from the 044 vector can creep into the peak corresponding to the 022 vector if the collimation is not tight enough. In this experiment it was very tight. We work with ratio $x=\frac{E_{\gamma}}{E_{0}}$ where $E_{\gamma}$ is the photon energy and $E_{0}$ is the energy of the radiating electron beam. The Bremsstrahlung spectrum is characterised by its discontinuous series of peaks with a vertical drop at the higher edge of the peak. This point is called the discontinuity point, $x_{g}^{d}$, where the superscript $d$ is used to label the point as the discontinuity point and $g$ is the lattice vector number. The following derivation for the fit used was developed by two other collaborators [109][110]. The polarization at a given energy, where $x<x_{g}^{d}$, is associated with the intensity at that energy:

$$
\begin{gathered}
I_{g}(x)=I_{g}^{0} \chi_{g}(x) \\
P_{g}(x)=\frac{-\phi_{g}(x)}{\left[1+\frac{I_{i}(x)}{I_{g}(x)}\right]}
\end{gathered}
$$

Which can be written as: 


$$
P_{g}(x)=-\phi_{g}(x) \times \frac{I_{g}(x)}{I_{\text {total }}(x)}
$$

Where:

- $I_{g}(x)$ is the coherent contribution from vector $0 \mathrm{gg}$.

- $P_{g}(x)$ is the corresponding polarization.

- $I_{g}^{0}$ is the peak intensity for vector $0 \mathrm{gg}$.

- $I_{i}(x)$ is the incoherent contribution.

- $\chi_{g}(x)$ and $\phi_{g}(x)$ are functions of $Q_{g}$.

- $Q_{g}=\frac{1-x_{g}^{d}}{x_{g}^{d}}$

$$
\begin{gathered}
\phi\left(x, x_{g}^{d}\right)=\frac{2 x^{2} Q_{g}^{2}}{1-x}\left[1+(1-x)^{2}-\frac{4 Q_{g}^{2} x^{2}}{1-x}\left(\frac{1-x}{Q_{g} x}-1\right)\right]^{-1} \\
\chi\left(x, x_{g}^{d}\right)=\frac{x^{2} Q_{g}^{2}}{1-x}\left[1+(1-x)^{2}-\frac{4 Q_{g}^{2} x^{2}}{1-x}\left(\frac{1-x}{Q_{g} x}-1\right)\right]
\end{gathered}
$$

The upper cut-off for a given coherent peak is at the discontinuity point, $x_{g}^{d}$, which is dependent on the angle between the beam and the crystal by the following relationship:

$$
x_{g}^{d}=\frac{2 \theta E_{0}\left(g_{2} \cos \alpha+g_{3} \sin \alpha\right)}{1+2 \theta E_{0}\left(g_{2} \cos \alpha+g_{3} \sin \alpha\right)}
$$

Where:

- $E_{0}$ is the energy of the radiating electron beam.

- $\theta$ and $\alpha$ are the polar and azimuthal angle of the electrom beam relative to the 022 lattice vector.

- $g_{2}$ and $g_{3}$ are the second and third lattice vector indices. 
Restricting this to cases where $g_{1}=0$ and $g_{2}=g_{3}$ simplifies the dependency of $x_{g}^{d}$ to just the polar angle:

$$
x_{g}^{d}=\frac{1}{\frac{k}{g E_{0}} \theta+1}
$$

Where:

- $k=m_{e} \frac{a}{4 \sqrt{2} \pi}=26.5601 \mathrm{MeV}$

- $m_{e}$ is the mass of the electron, $0.511 \mathrm{MeV}$.

- $\mathrm{a}$ is the diamond lattice constant, 923.7 unitless.

Equation 5.10 shows that, for a fixed electron beam energy, $E_{0}$, we can adjust the position of the coherent edge, $x_{g}^{d}$, by altering the small angle, $\theta$, between the beam and the 022 lattice vector. If we substitute equation 5.10 into $Q_{g}=\frac{1-x_{g}^{d}}{x_{g}^{d}}$, equations 5.7 and 5.8 become functions of $\mathrm{x}, \mathrm{g}$, and $\theta$ :

$$
\begin{gathered}
\phi(x, g, \theta)=\frac{2 x^{2} Q_{g}^{2}}{1-x}\left[1+(1-x)^{2}-\frac{4 Q_{g}^{2} x^{2}}{1-x}\left(\frac{1-x}{Q_{g} x}-1\right)\right]^{-1} \\
\chi(x, g, \theta)=\frac{x^{2} Q_{g}^{2}}{1-x}\left[1+(1-x)^{2}-\frac{4 Q_{g}^{2} x^{2}}{1-x}\left(\frac{1-x}{Q_{g} x}-1\right)\right]
\end{gathered}
$$

The beam is collimated as the coherent radiation is more focused than the incoherent radiation so the collimation increases the enhancement.

fractional uncertainty of the polarization:

$$
\frac{\Delta P}{P}=\frac{1}{(\xi-1)} \times \frac{\Delta \xi}{\xi}
$$

Equations 5.11 and 5.12 need to expanded to account for the smearing of the beam divergence and position over the collimator, and the smearing og the angle between the beam and the crystal. Equations 5.14 and ?? show the modified forms which are used 
to fit the enhancements and to determine the associated polarization.

$$
\xi(x, \theta, \sigma)=\frac{\int_{\theta-3 \sigma}^{\theta+3 \sigma}\left\{e^{\frac{\left(\theta^{\prime}-\theta\right)^{2}}{2 \sigma^{2}}}\left[1+\frac{I_{0} \times C\left(x, \theta^{\prime}\right) \times \chi\left(x, \theta^{\prime}\right)}{I_{a m o}(x)}\right]\right\} d \theta^{\prime}}{\int_{\theta-3 \sigma}^{\theta+3 \sigma}\left\{e^{\frac{\left(\theta^{\prime}-\theta^{2}\right.}{2 \sigma^{2}}}\right\} d \theta^{\prime}}
$$

The expression for the polarization contains contributions from the multiple $\theta$ positions at each $\mathrm{x}$ value with their individual weight, intensity, and polarization and this gives the following form:

$$
P(x, \theta, \sigma)=\frac{\int_{\theta-3 \sigma}^{\theta+3 \sigma}\left\{e^{\frac{\left(\theta^{\prime}-\theta\right)^{2}}{2 \sigma^{2}}} \times \phi\left(x, \theta^{\prime}\right) \times I_{c o h}\left(x, \theta^{\prime}\right)\right\} d \theta^{\prime}}{\int_{\theta-3 \sigma}^{\theta+3 \sigma}\left\{I_{c o h}\left(x, \theta^{\prime}\right) \times e^{\frac{\left(\theta^{\prime}-\theta^{2}\right.}{2 \sigma^{2}}}\right\} d \theta^{\prime}}
$$

The fitting procedure is based on the following five parameters:

- $\theta$ - the angle between the radiating electron beam and the 022 lattice vector.

- $\sigma$ - the Gaussian smearing of $\theta$ which accounts for small fluctuations in $\theta$ associated with beam divergence and multiple scattering.

- $\theta_{r}$ - the relative angle of the collimation related to the angle of collimation, $\theta_{c}$ by the following expression, $\theta_{r}=\theta_{c} E_{0}$.

- $\sigma_{r}$ - the smearing of the photon beam spot across the collimator.

- $I_{0}$ - the intensity amplitude of the 022 coherent peak.

The enhancement spectra in the region of the 022 coherent edge are fitted using equation 5.14 and the corresponding polarization distribution is described by equation 5.15. In order to apply this fit, reasonable values are needed to initiate the fit parameters. This is easily done as they can be deduced by known experimental values. Knowing the radiating electron beam's energy, the value of the current coherent edge, the diameter of the collimator, and the distance of the collimator from the radiator, allows us to calculate first estimations for the fit parameters, e.g.: 


$$
\theta=\frac{k}{2 E_{0}\left[\frac{1}{E d g e_{c o h}}-\frac{1}{E_{0}}\right]}
$$

Where $\mathrm{k}$ is $26.5601 \mathrm{MeV}$ as defined for equation 5.10, $E_{0}$ and $E d g e_{c o h}$, are the energies of the electron and the coherent edge, respectively. By studying the enhancement spectrum we can deduce $\sigma$ as this manifests itself in the gradient of the higher energy slop of the peak, and similarly $\sigma_{r}$ for the lower energy slop of the peak. The following section will describe how this process is used in detail.

\subsection{Fitting}

For every nominal coherent edge setting a 2-D histogram is created showing the measured coherent edge position against the enhancement spectra. This is then sliced into $2 \mathrm{MeV}$ bins of the measured coherent edge position and the corresponding enhancements for each bin is fitted. Therefore a polarization table for each measured edge position is attained.

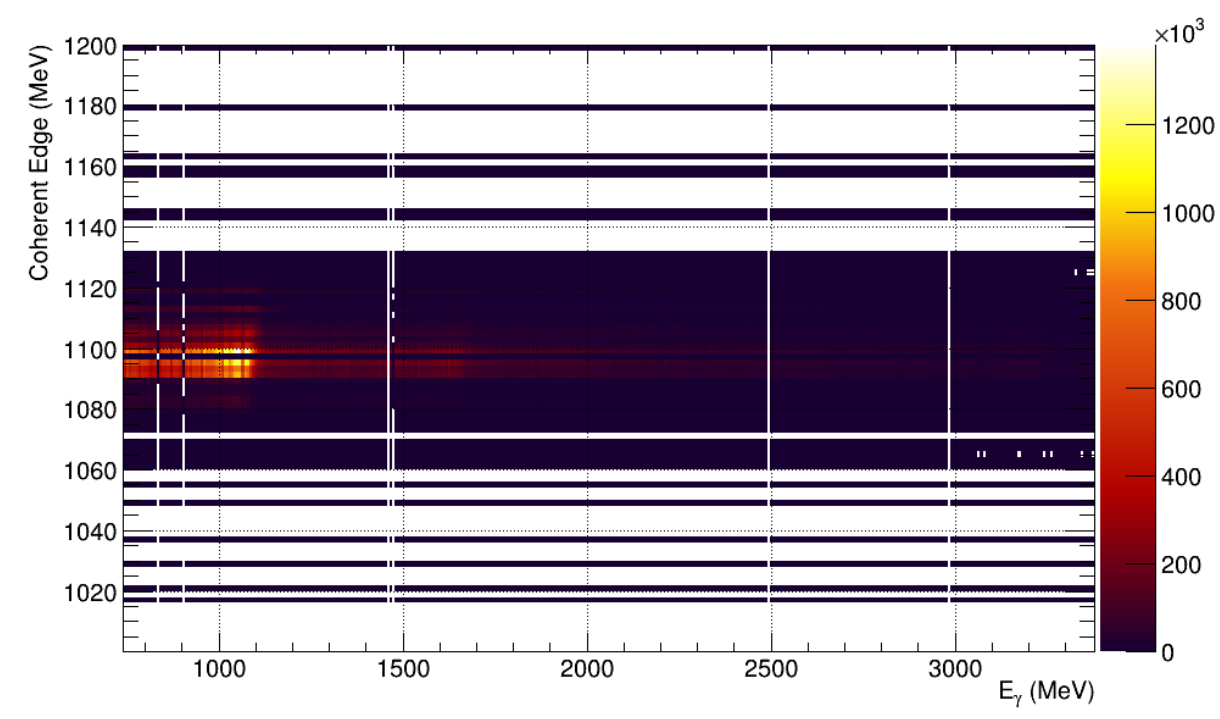

Figure 5.11: The corrected parallel polarization energy spectrum as a function of the measured coherent edge position for the $1.1 \mathrm{GeV}$ data used for attaining enhancement spectra for the different measured coherent edge positions that the beam was at during the running of the experiment. 
Figure 5.11 shows the measured coherent edges against the photon energy from the dataset when the edge was nominally set at $1.1 \mathrm{GeV}$ and a large deviation from that value can be seen. Figure 5.12 shows the various values of the coherent edge for the parallel polarized data for the same dataset and shows how much $\theta$ changes throughout one dataset clearly.

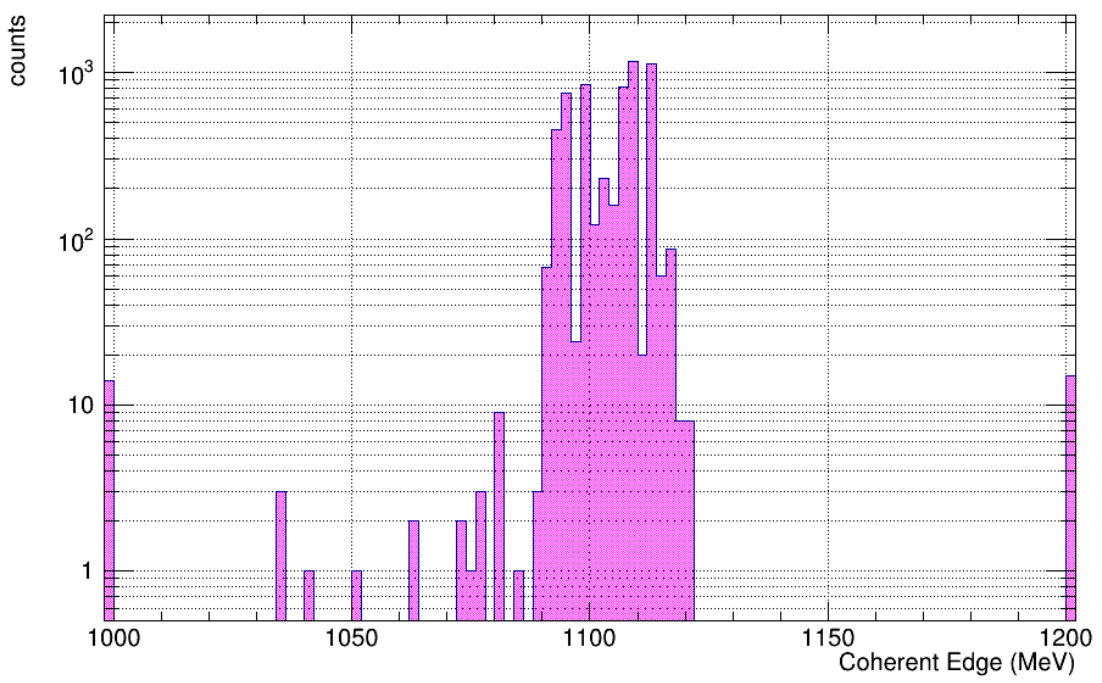

Figure 5.12: The differenet measured coherent edge values for the $1.1 \mathrm{GeV}$ coherent edge dataset for the parallel polarization data.

The data is divided in $2 \mathrm{MeV}$ bins of the measured coherent edge position and individual enhancement spectra for each $2 \mathrm{MeV}$ are generated using the technique described above. Figure 5.1 shows a typical enhancment spectrum in its entirety for one of these $2 \mathrm{MeV}$ bins. But as described above we are only interested in the first peak which is fitted using equation 5.14. An example of this is shown in 5.13 where the top panel shows the fitted peak and the bottom shows the corresponding polarization distribution using equation 5.15. Noisy and low statistic spectra are discarded at this part in the process and the remaining good fits have their fit parameters plotted against the measured coherent edge corresponding the relative spectra. This is shown in 5.14. A second degree polynomial is then applied to each parameter and the corresponding value for each spectra is used to rescale each enhancement spectra and are used as the starting values for the second application of the fit. This rescaling is necessary as, by 
their very nature, the areas used to set the baseline have poor statistics and therefore are much less reliable and more susceptible to any differences between the experimental variables when using the diamond radiator and the amorphous radiator. The following expression was used to rectify problems with scaling:

$$
k_{\text {rescale }}=\frac{\int_{\text {low }}^{\text {high }} \xi_{f i t}}{\int_{\text {low }}^{\text {high }} \xi_{\text {data }}}
$$

Where the integral boundaries are shown in 5.13 by the magenta lines. Where an individual enhancement had a poor fitting function (too high a $\chi^{2}$ ) the fit parameters were take from the second degree polynomial fits of the parameters versus coherent edge, which only used parameters from successful fits, and the corresponding fit integrals were calculated using those values rather than the real parameters from the failed fits. 

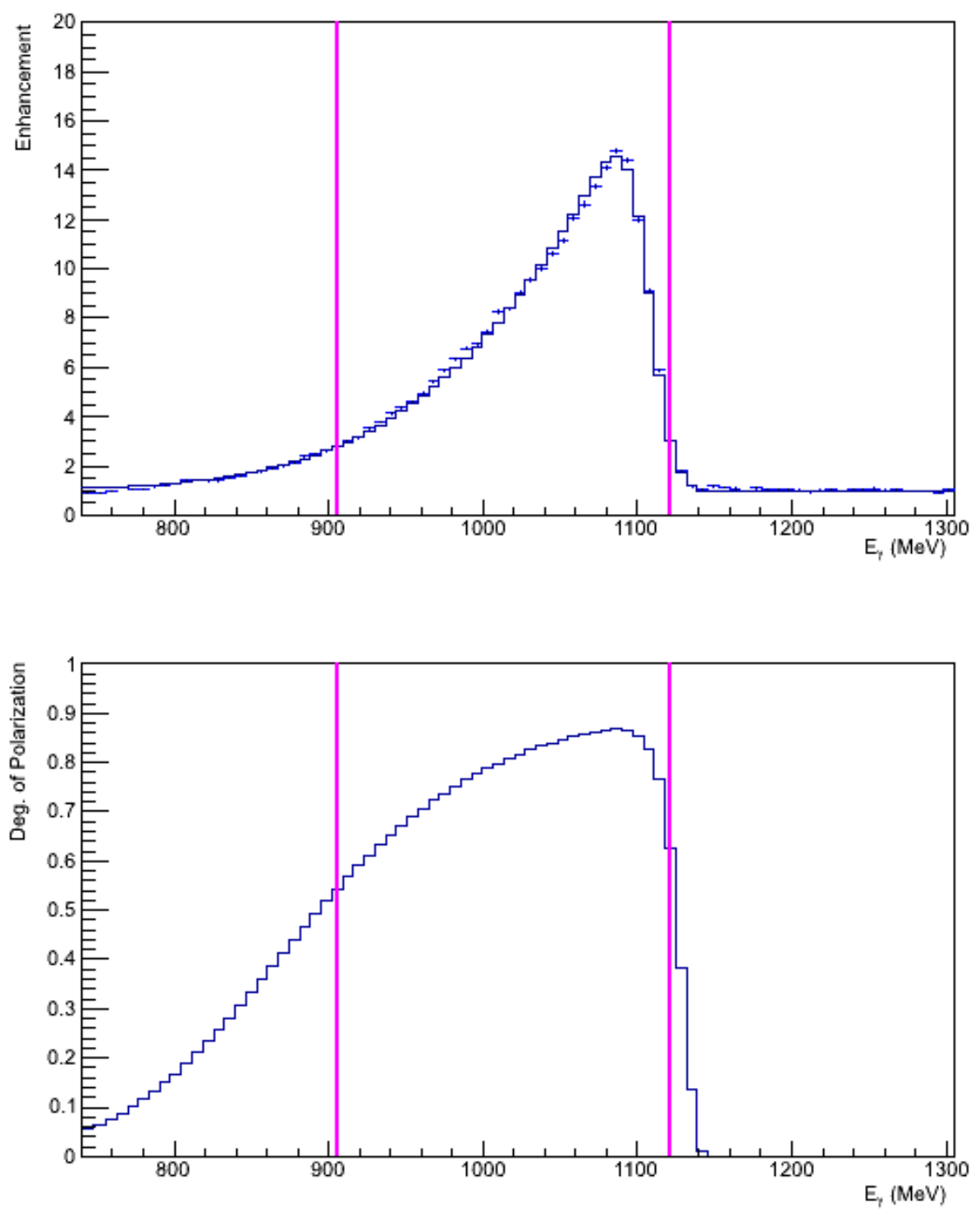

Figure 5.13: First pass of fitting the enhancment plot for data taken during the g9a experiment with the initial electron beam energy at $3.5 \mathrm{GeV}$ and the coherent edge set at $1.1 \mathrm{GeV}$. This plot shows the fit for a specific measured coherent edge and the corresponding polarization. 

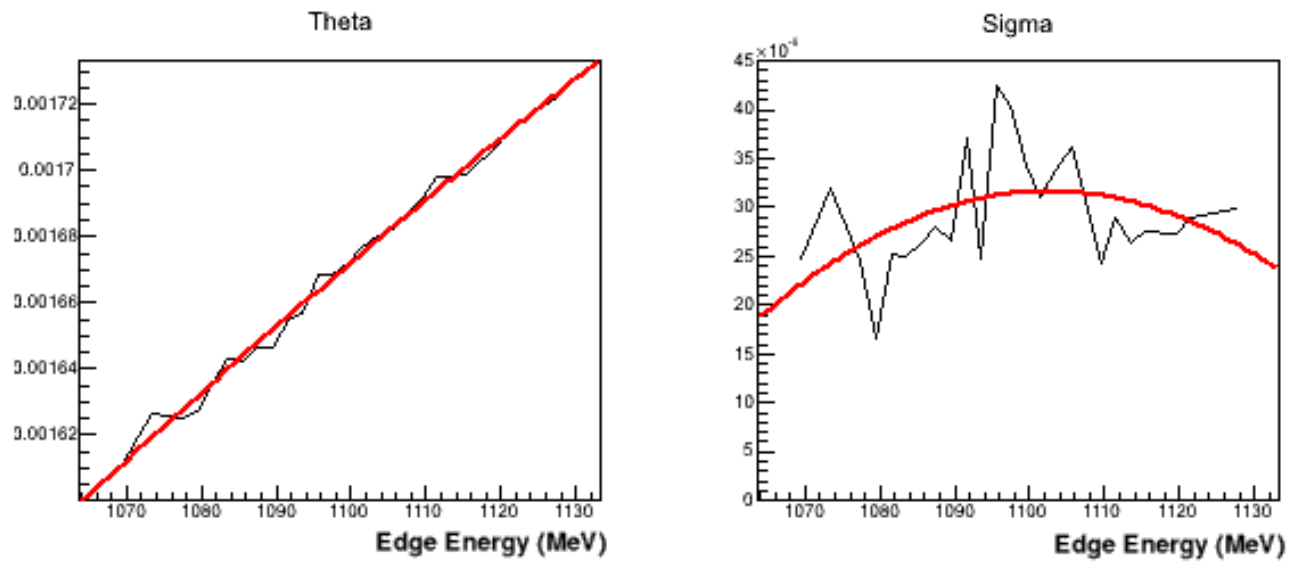

ThetaR
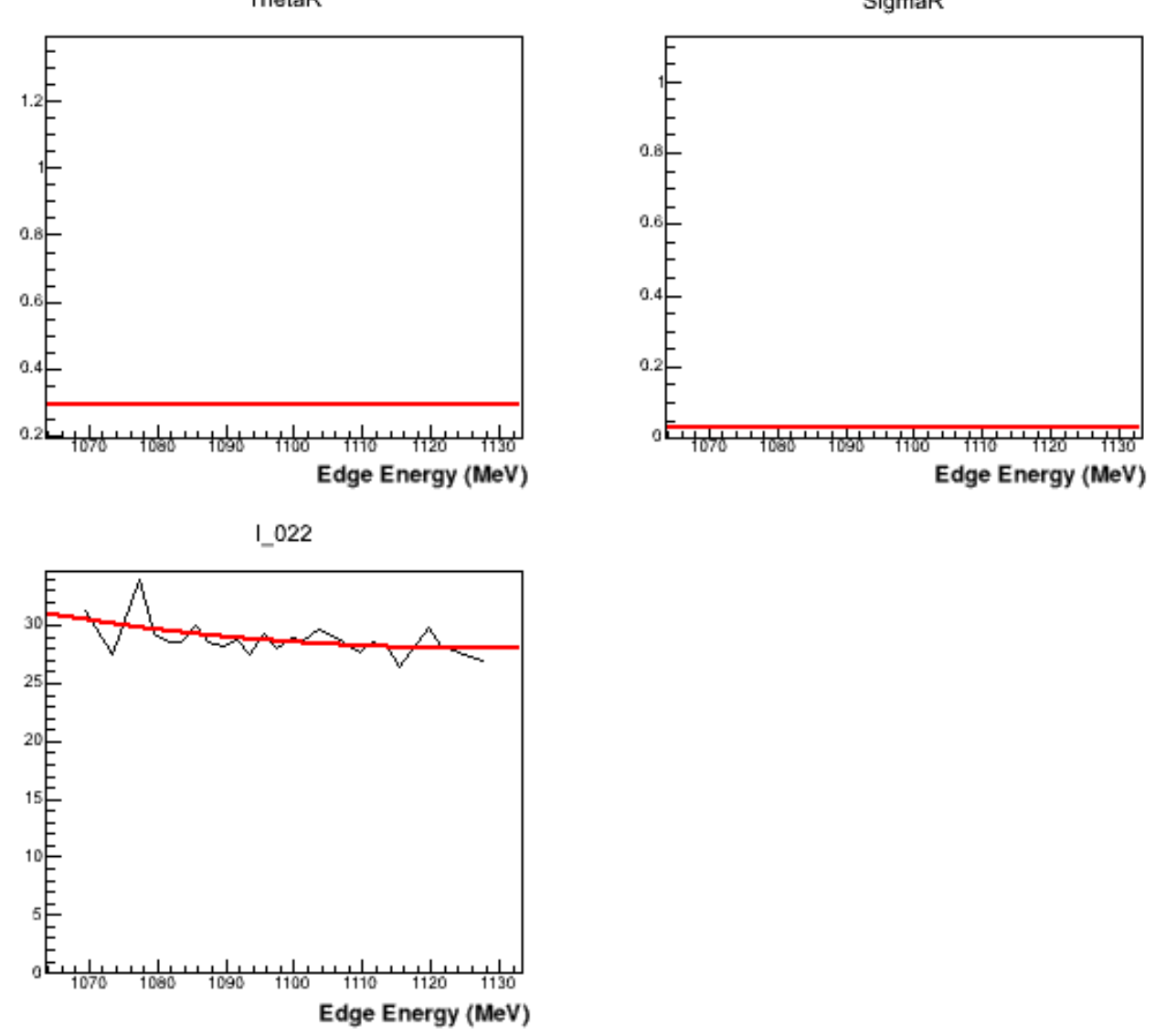

Figure 5.14: Fit parameters from the first application of the fit. Spectra with a poor fit are discarded for this process. A 3-degree polynomial is applied to the fit parameters and the values from this fit are used in the second application of the fit to help with rescaling low statistic and noisy spectra. $\Theta_{r}$ and $\sigma_{r}$ are fixed values hence the lack of deviation.

After each enhancement is rescaled the fit is reapplied. Discrepancies between the fit and the enhancement are taken into consideration by using equation 5.13. Most often the discrepancy, excluding low statistic enhancements being problematic to fit, 
is near the top of the peak. This is not a great problem as the higher the enhancement, the smaller the change in polarization. However, these small differences are taken into consideration. Figure 5.15 shows the fit applied to the rescaled enhancment in the top panel, and the corresponding polarization distribution. The original polarization values taken from the fit are shown in green and the corrected values are shown as blue points. It is further presumed that the change in polarization for any given tagger counter ought to be smooth. The reader is reminded that each tagger counter corresponds to an individual photon energy. So for every tagger counter the polarization values are plotted against the different measured coherent edges and a polynomial fit is applied. An example is shown in figure 5.16. Every polarization point is then recalculated using the fit parameters from these plots. These final correction are shown in the second panel of figure 5.15 as the magenta line. 

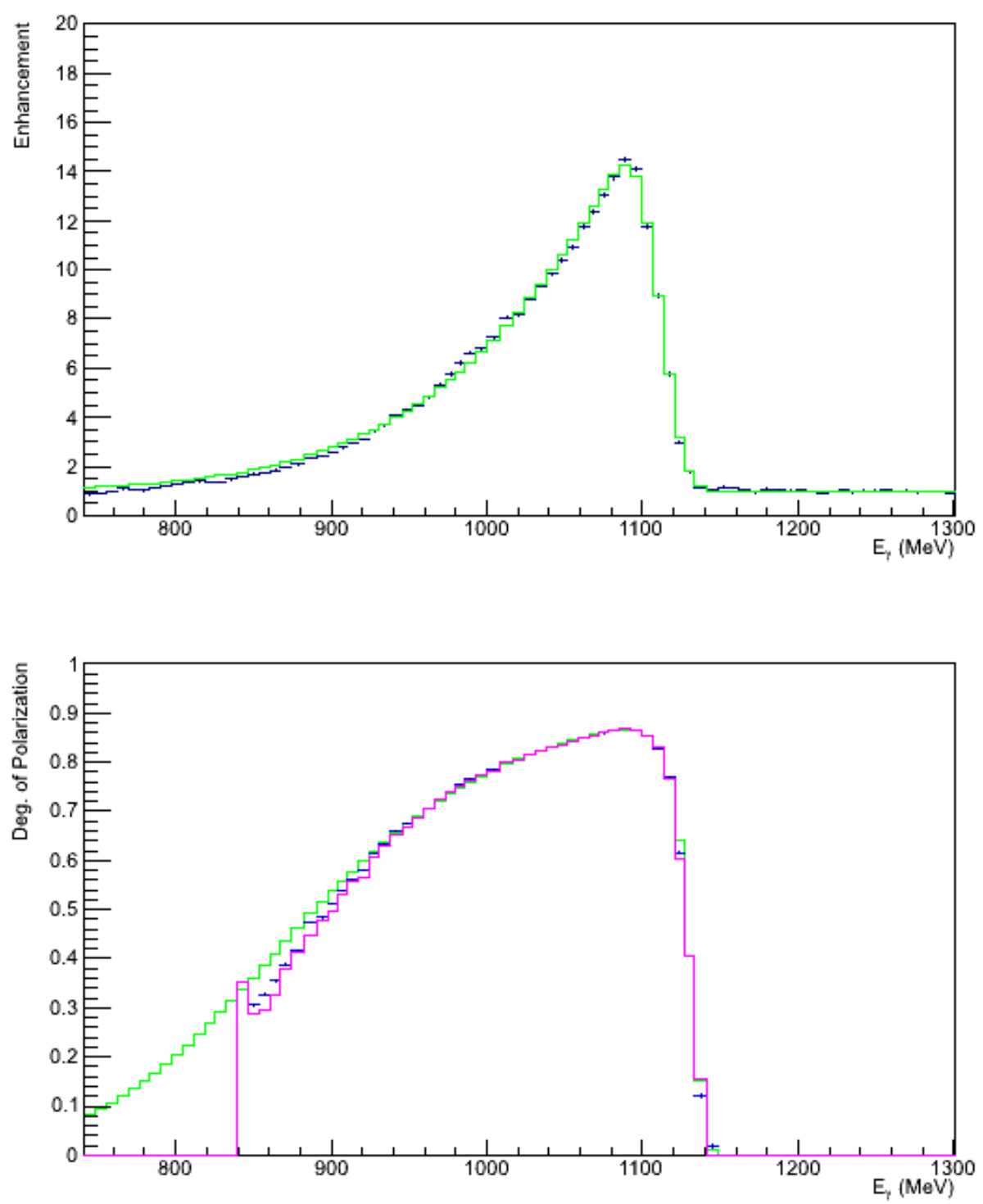

Figure 5.15: Second pass of fitting the enhancment plot for same data in the previous figure. This plot shows the fit for a specific measured coherent edge and the corresponding polarization after the rescalling of the baseline. 


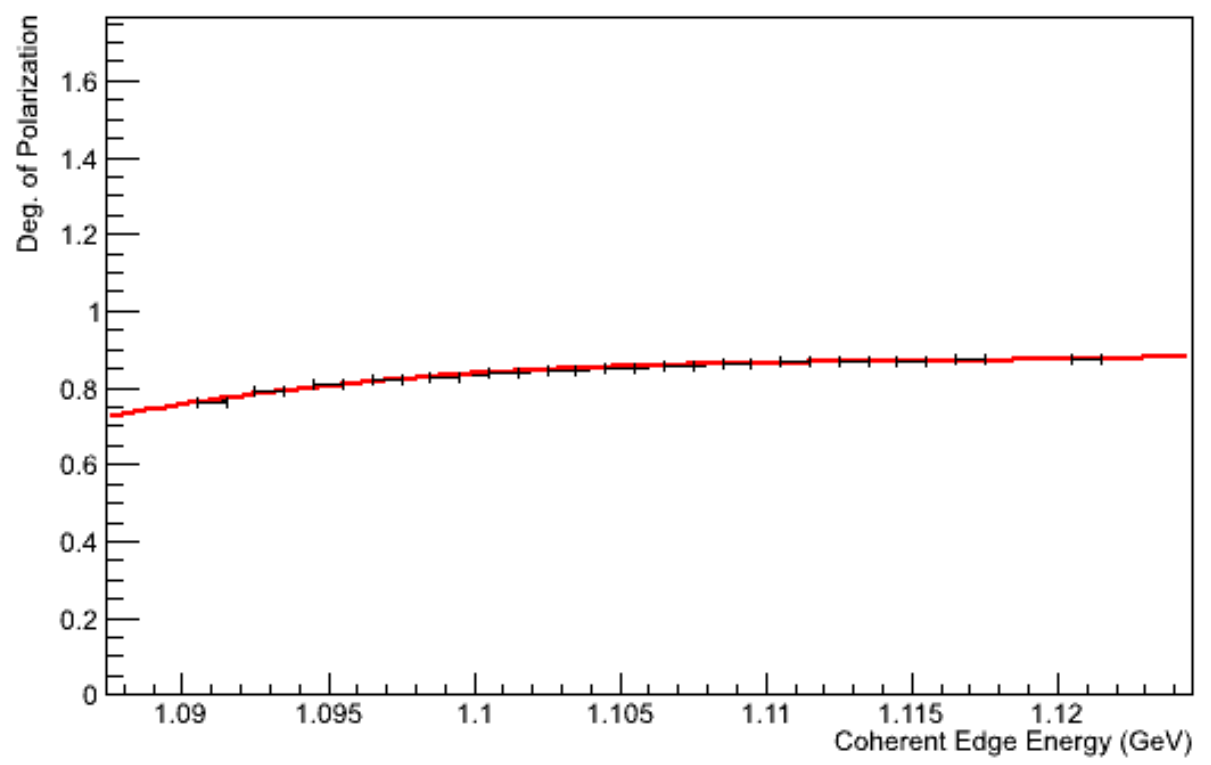

Figure 5.16: The polarization values as a function of coherent edge position for a specific e-counter corresponding to $E_{\gamma}=1103 \mathrm{MeV}$ with the same settings described in the figures above.

These values, the initial polarization, the corrected, and the smoothed, are all written into a table along with the corresponding information needed to use them, the estimated uncertainty and the corresponding measured coherent edge. The final section will describe how the tables for $\mathrm{g} 8 \mathrm{~b}$, the dataset used in the main analysis of this thesis, were used. 


\subsection{Polarization Tables}

\begin{tabular}{|c|c|c|c|c|c|c|c|c|}
\hline$E_{I D}$ & $E_{\gamma}$ & $\xi_{\text {data }}$ & $\sigma_{\xi}$ & $\xi_{\text {fit }}$ & $P_{\text {fit }}$ & $P_{\text {corr }}$ & $P_{\text {corr }}^{\text {err }}$ & $P_{\text {smoothed }}$ \\
\hline 356 & 1206.2 & 11.117 & 0.142 & 12.362 & 0.851 & 0.849 & 0.000 & 0.850 \\
\hline 355 & 1215.3 & 12.461 & 0.156 & 13.340 & 0.859 & 0.860 & 0.000 & 0.861 \\
\hline 354 & 1224.4 & 13.632 & 0.171 & 14.353 & 0.866 & 0.868 & 0.000 & 0.869 \\
\hline 353 & 1233.4 & 14.185 & 0.171 & 15.354 & 0.872 & 0.873 & 0.000 & 0.876 \\
\hline 352 & 1242.4 & 14.717 & 0.173 & 16.288 & 0.877 & 0.876 & 0.000 & 0.884 \\
\hline 351 & 1251.3 & 16.533 & 0.195 & 17.141 & 0.881 & 0.884 & 0.000 & 0.892 \\
\hline 350 & 1260.4 & 17.134 & 0.199 & 17.702 & 0.884 & 0.887 & 0.000 & 0.898 \\
\hline 349 & 1269.3 & 17.649 & 0.206 & 17.803 & 0.885 & 0.889 & 0.000 & 0.899 \\
\hline
\end{tabular}

Table 5.1: Part of the polarization table for $\mathrm{g} 8 \mathrm{~b}$ for the dataset with the coherent edge nominally set at $1300 \mathrm{MeV}$. This section shows the tagger counters in the vicinity of the enhancement peak. The columns show, in order, the tagger counter channel number, the corresponding photon energy, the enhancement for that channel, the enhancement error, the enhancement from the fit, the polarization from the fit, the corrected polarization, the corrected polarization error, and finally the smoothed polarization. The smoothed polarization value is the one used when undertaking analysis.

Table 5.1 shows a subsection of a typical polarization table used in analysis using data from the g8a experiment. Optimally the table would be looked up on an event-byevent basis. The multiplicity is usually in the order of $\sim 15$ photons per event so the most likely photon detected must be chosen for a given event by looking at the timing coincidence. This processes is described below in section 6.1.5. Once a photon is chosen the table corresponding to the last measured coherent edge is accessed and the values from the row of the tagger counter associated with the chosen photon's energy are read in. The polarization is then added to that event's data.

However, this requires the current measured coherent energy being accurate and sometimes the online fitting procedure failed. This resulted in a coherent edge measurement which is outside the range of the polarization table. In order not to just 
discard these events a more robust method was adopted where the events are clustered together in groups of 10,000 events. The coherent edge position does not fluctuate over such a short period of time and so the first event's coherent edge setting is used if it is a rational value, if not the previous cluster's value is used and the next event will be used for setting the new cluster's coherent edge or until an event with a suitable value.

An independent study was undertaken to deduce the systematic uncertainty of the polarization values calculated for the $\mathrm{g} 8 \mathrm{~b}$ set. It was shown that a small alteration of the calculated value was required; $4 \%$ for the $1.3,1.5,1.7$, and $1.9 \mathrm{GeV}$ datasets and a slightly larger value for the $2.1 \mathrm{GeV}$ of $6 \%$. In addition to this correction it was shown that the optimum energy range to use was $E_{\text {edge }}-200 \mathrm{MeV}<E_{\gamma}<E_{\text {edge }}$ otherwise the systematic uncertainty rose to unacceptable levels [111][112].

The table below shows the average polarization values for the different run settings in g8a after the most likely prompt photon has been chosen in order to allow the reader an idea of the typical polarization levels the above process begets.

\begin{tabular}{|c|c|c|}
\hline Coherent Edge $(\mathrm{GeV})$ & $P_{\|}$ & $P_{\perp}$ \\
\hline 1.3 & $82.5 \%$ & $82.1 \%$ \\
\hline 1.5 & $82.9 \%$ & $81.7 \%$ \\
\hline 1.7 & $77.0 \%$ & $76.2 \%$ \\
\hline 1.9 & $71.0 \%$ & $70.6 \%$ \\
\hline 2.1 & $60.0 \%$ & $66.6 \%$ \\
\hline
\end{tabular}

Table 5.2: Average polarization values for the different run settings for the g8b dataset after the best photon process has selected an individual photon for each event. 


\section{Chapter 6}

\section{Data Analysis}

In this chapter the analysis of the calibrated data is presented. The first section describes the initial process of selecting candidate events. The complexity of selecting the $\rho^{0}$ channel is then discussed together with how the uncertainty in the extracted asymmetry. This is followed by a brief discussion on the effects of cutting on the $\Delta^{++}$and the $\Delta^{0}$ and how the regions for the kinematic binning were chosen. Then the phenomenological fitting procedure used to highlight $\rho^{0}$ and $f^{0}$ regions in the invariant mass distributions of the two pions is described. The subsequent section deals with the fitting procedure developed to extract the beam asymmetry for the region of $M\left(\pi^{+} \pi^{-}\right)$associated with the $\rho^{0}$ meson. The procedure is developed from a standard one-dimensional fit developed for pseudo-scalar analyses and takes into consideration the tensor sensitivity of vector mesons. The final section describes the beam asymmetry extraction for the $f^{0}$ meson and in addition to this the extraction of the beam asymmetries for the individual decay pions analagous to the analysis done by the CBELSA collaboration for the $\omega$ meson [113].

\subsection{Particle Identification}

The first step in the analysis procedure is to choose the detected events which are associated with the process of interest. Loose cuts are applied to cooked data from the 
DAQ, as described in section 4.8, while it is converted into a format more easily analysed using the ROOT package from CERN. Any event with at less than two charged particles are discarded. From there a series of procedures are used to select events specific to the process of interest. The majority of these procedures follow the same protocol from the analysis on strangeness photoproduction using the same dataset as here [114].

\subsubsection{First Cuts}

To analyse the $\pi^{+} \pi^{-}$across as much phase space as possible it is necessary to study the exclusive and semi-inclusive channels. This is due to the forward angle dominance of this reaction channel and the fact that there is a hole in the detector for inserting different targets.

When looking at the angular distributions in the helicty frame, as described in detail ??, in order to extract spin density matrix elements there is an artificial hole in the distribution. The helicity frame is fundamentally the rest frame of the reconstructed $\rho^{0}$ meson which means that the target insertion hole affects the distribution of the decay pions as described above. By extending our analysis to include events where only a proton and one of the charge pions is detected or when only two oppositely charged pions are detected, we can cover a larger phase space. Hence, events with all the three final state charged particles or any of the permutations of two of the three particles are retained.

After events with either two or three charged particles are selected, intitial loose cuts are applied to the masses of the detected particles. They are not restrictive because the calculated mass from the cooking is a first approximation. The values used are displayed in table 6.1. These are purposefully left very loose as the following processes restrict the accepted particles more thoroughly.

The next stage after the initial events are selected is the beta versus momentum cut. 


\begin{tabular}{|l|c|r|}
\hline & $\pi^{+} / \pi^{-}$ & Proton \\
\hline Lower Limit(GeV) & 0.00 & 0.49 \\
\hline Upper Limit(GeV) & 0.20 & 1.44 \\
\hline
\end{tabular}

Table 6.1: The values used for the soft cuts on the mass of the detected particles.

\subsubsection{Beta Versus Momentum Cuts}

This process allows us to select the particles which most closely represent the nature of the particles of interest, much more so than simple mass cuts of the first section.

In figure 6.1 distinct tracks can be seen representing all of the individual particles associated with this reaction channel. However, there is also a large amount of background in these plots. In order to remove this background we look at the difference between the measured beta for each particle and the corresponding calculated beta for that particle type using equation 6.1

$$
\Delta \beta=\beta_{\text {meas. }}-\frac{k_{\text {meas. }}}{\sqrt{k_{\text {meas. }}^{2}+m_{P D G}^{2}}}
$$

Where:

- $\beta_{\text {meas. }}$ is the measured beta of the detected particle.

- $k_{\text {meas. }}$ is the measured momentum of the detected particle.

- $m_{P D G}$ is the mass of the detected particle taken from the Particle Data Group.

This difference between the measured beta and the theoretical value for each particle type has momentum dependent cuts applied to it. The beta difference as a function of momentum before the cuts is shown in 6.2 and after the cuts are applied in 6.3. The effect this procedure has can be seen in 6.4. At this point the detected particles are considered to be either protons or pions and therefore have their mass set to the values given by the Particle Data Group and any corresponding values are re-calculated using this value, e.g. the beta of each particle.

The beta verus momentum cuts for the reconstructed missing particles happen after the missing mass cuts. They need to be done first in order to identify potential 


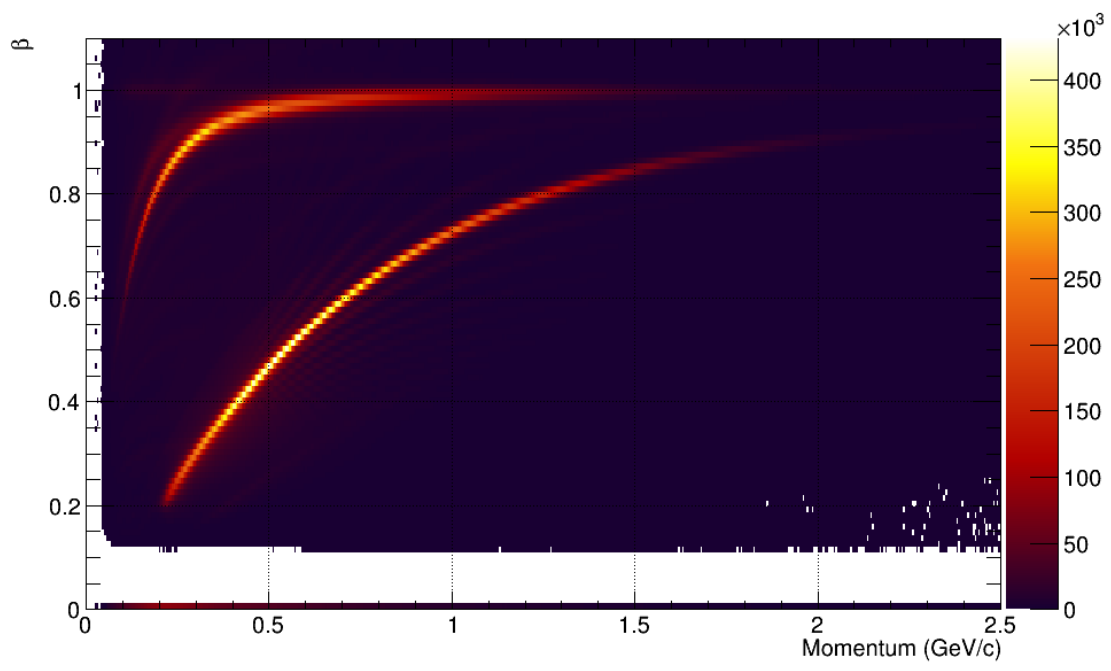

(a)

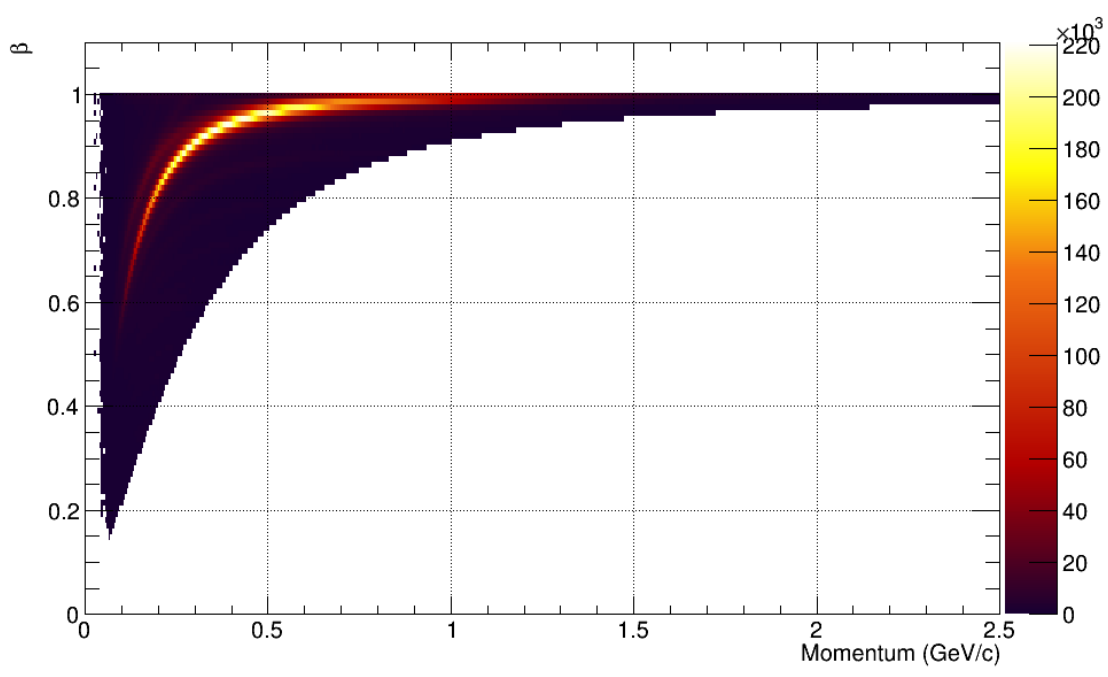

(b)

Figure 6.1: The measured beta of the detected particles as a function of the momentum for a) the positive detected particles and b) the negative detected particles. Distinct tracks can be seen corresponding to the pions and the proton (the lower beta track in the positive particle plot). 


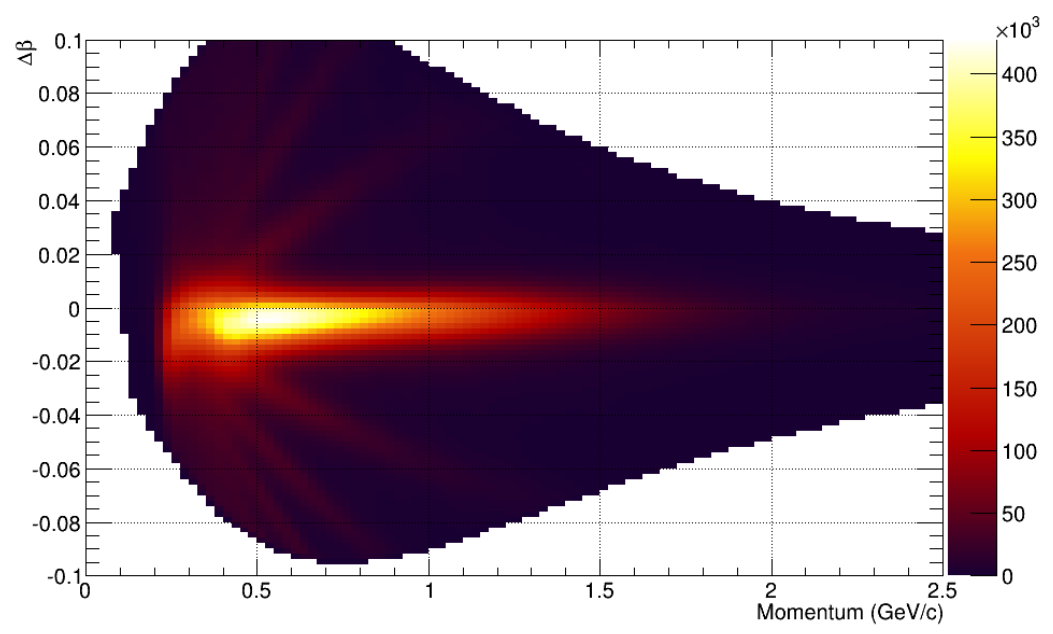

(a)

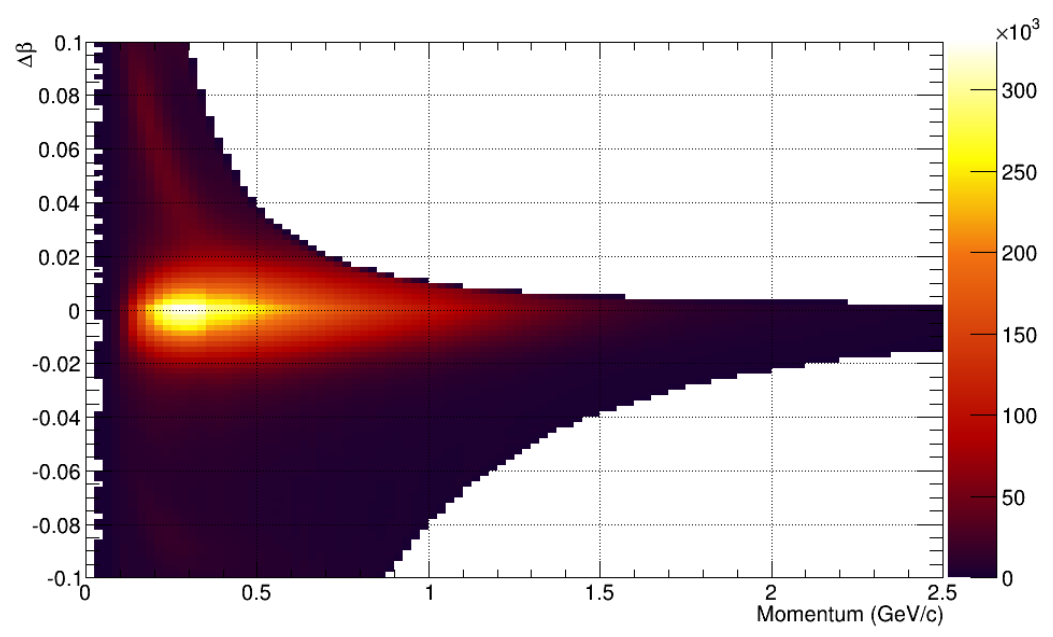

(b)

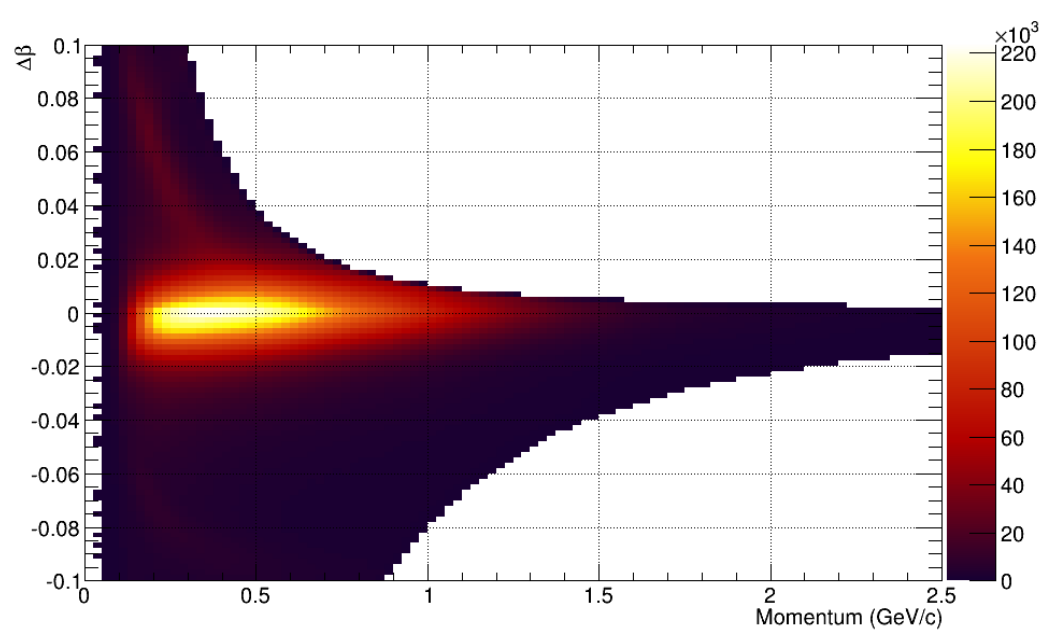

(c)

Figure 6.2: The difference between the measured beta of the detected particles and the calculated value for the corresponding expected particle type as a function of the momentum for a) the proton b) positive pion and c) the negative pion. 


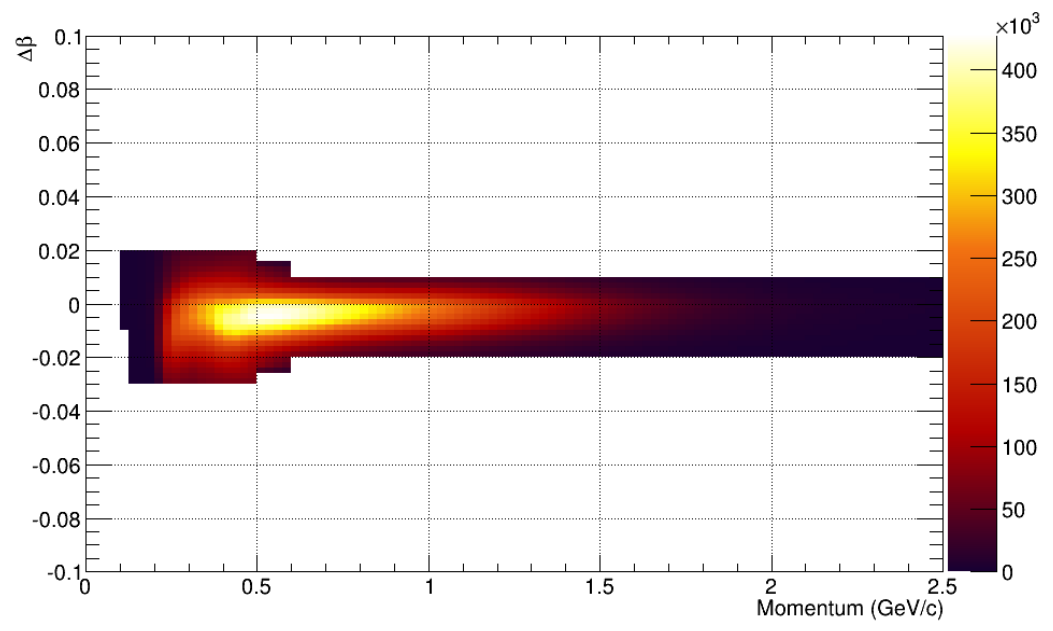

(a)

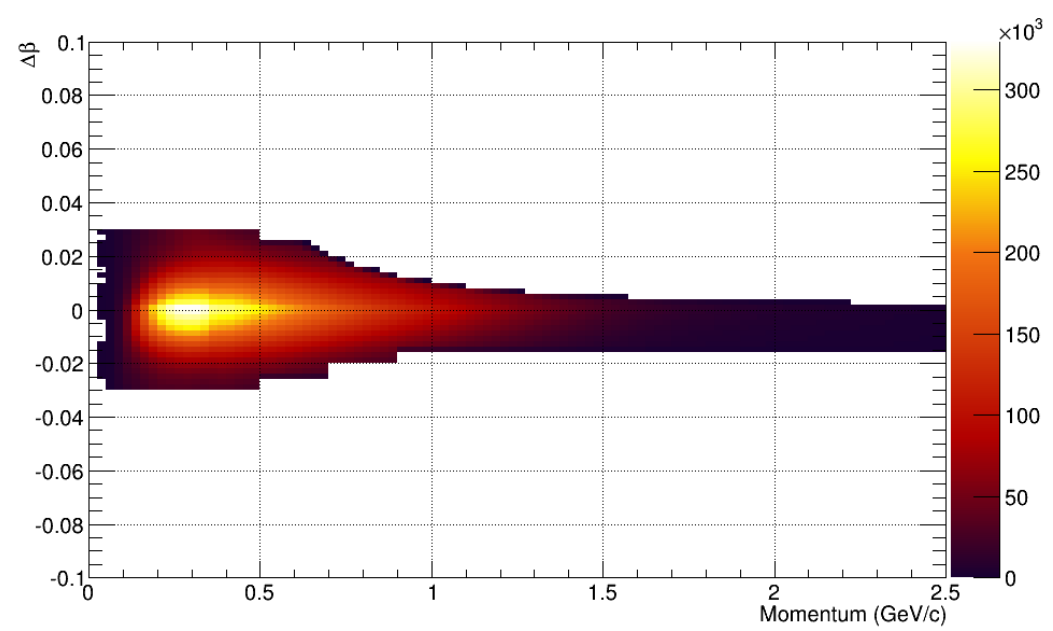

(b)

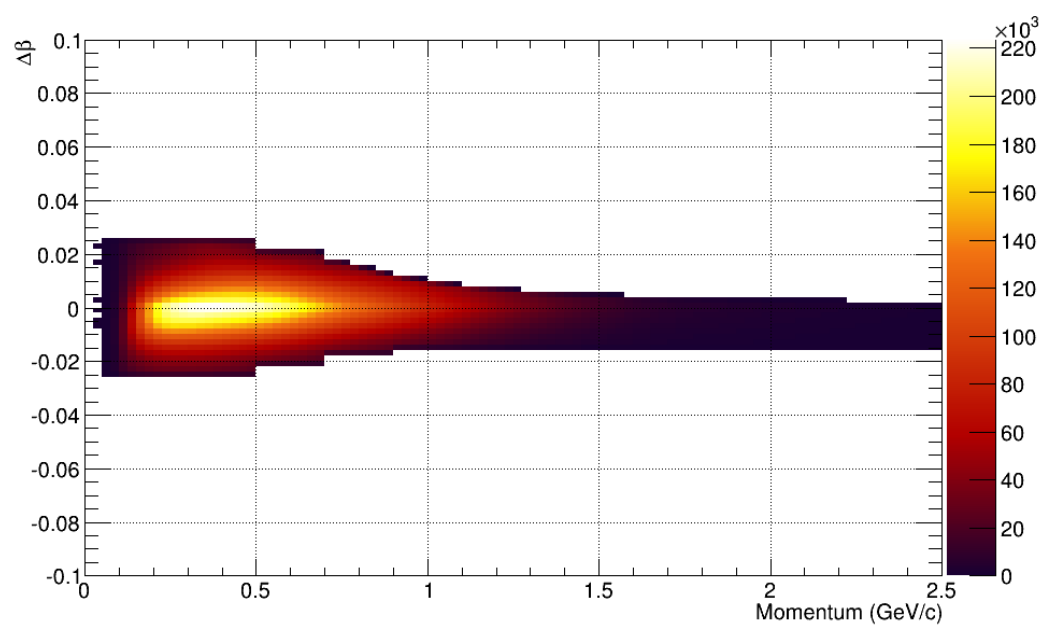

(c)

Figure 6.3: The difference between the measured beta of the detected particles and the calculated value for the corresponding expected particle type as a function of the momentum with the cuts applied for a) the proton $b$ ) positive pion and c) the negative pion. 


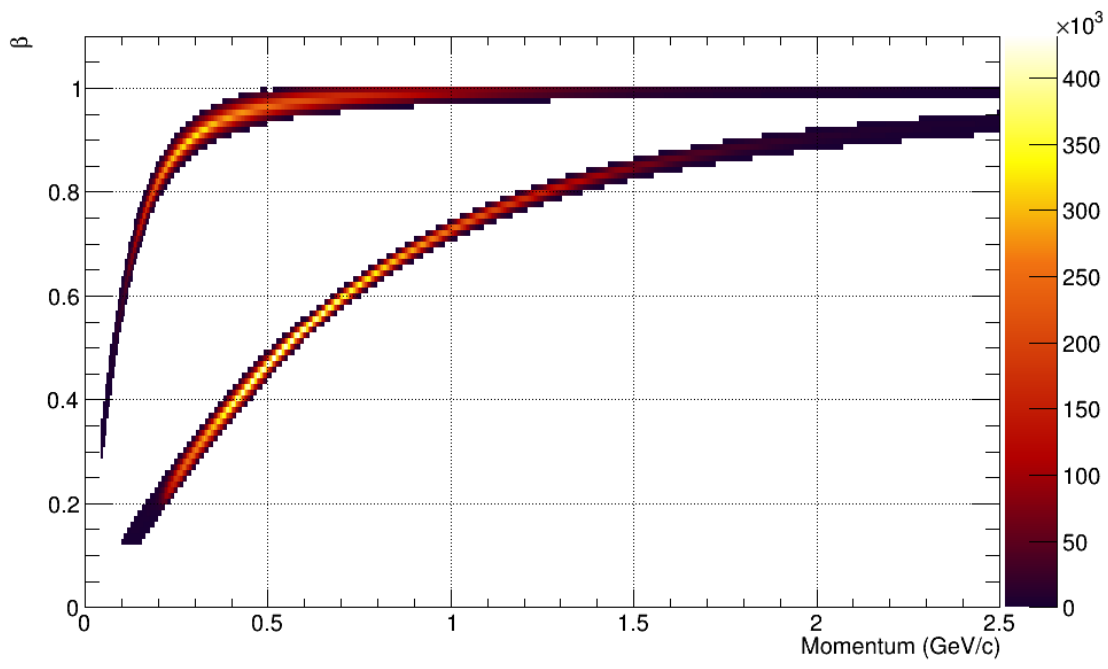

(a)

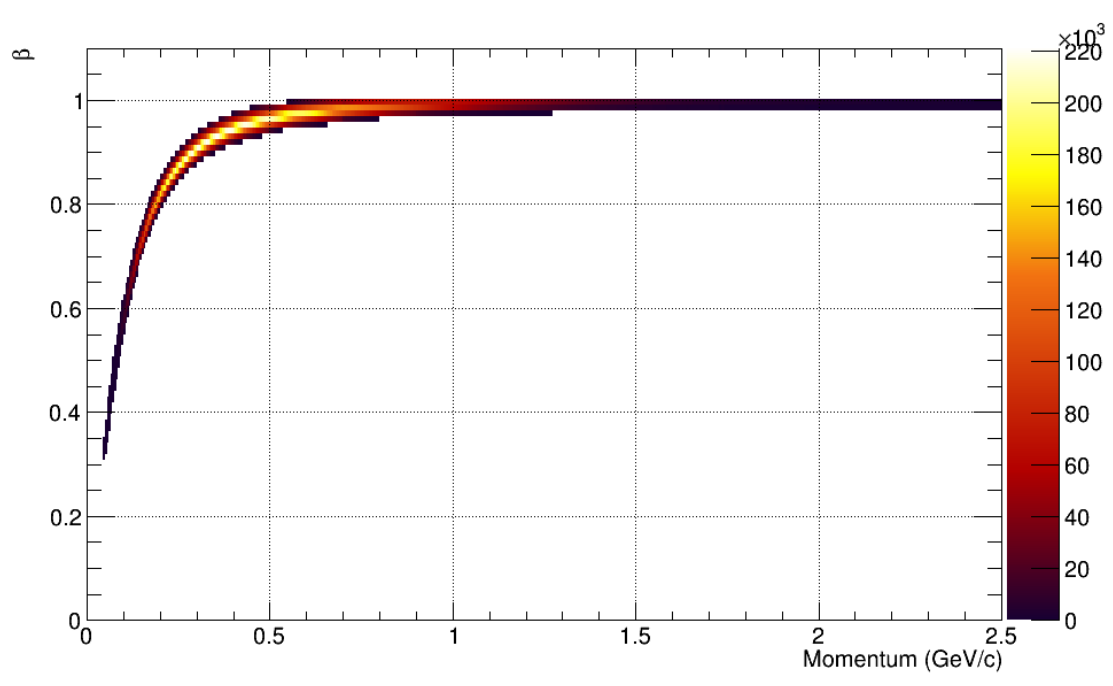

(b)

Figure 6.4: The measured beta of the detected particles as a function of the momentum after the $\Delta \beta$ cuts for a) the positive detected particles and b) the negative detected particles. The majority of the background has been removed in comparison to 6.1. 
candidates similar to the loose mass cuts of the initial stage for the detected particles.

\subsubsection{Z-vertex}

Once we have events with the right particle topology we need to check that they originated in the target cell. The first cut is on the detected particles' z-vertex so that we can remove events with particles outside the target geometry. As described in section 4.5, the target is $40 \mathrm{~cm}$ long so the cut is applied between 0 and $40 \mathrm{~cm}$. The cut is shown in 6.5

\subsubsection{Fiducial Cuts}

The events detected in the areas near the holes in the detector between the six regions are removed at this point. Figure 6.6 shows the polar angle as a function of the azimuthal angle of each of the three final state particles and the fiducial regions are obvious. The particles detected near these regions carry a higher stastical uncertainty. As such a $\theta$-dependent cuts are applied in these regions. The value used are shown in 6.2. The angular distribution of the particles is shown in figure 6.7.

\begin{tabular}{|l|c|r|}
\hline$\theta<10$ & $10 \leq \theta<20$ & $\theta \geq 20$ \\
\hline $\pm 8^{\circ}$ & $\pm 5^{\circ}$ & $\pm 2^{\circ}$ \\
\hline
\end{tabular}

Table 6.2: The values used for fiducial cuts.

As was the case with the beta versus momentum the angular distributions for the reconstructed missing particles are created after the missing mass cuts. However, no cuts are applied to these distributions and so they are displayed here to show how they compare with the detected particles' angular distribution.

Figure 6.8 shows these distributions and highlights the need for the semi-inclusive topologies in order to cover as much phase space as possible. 


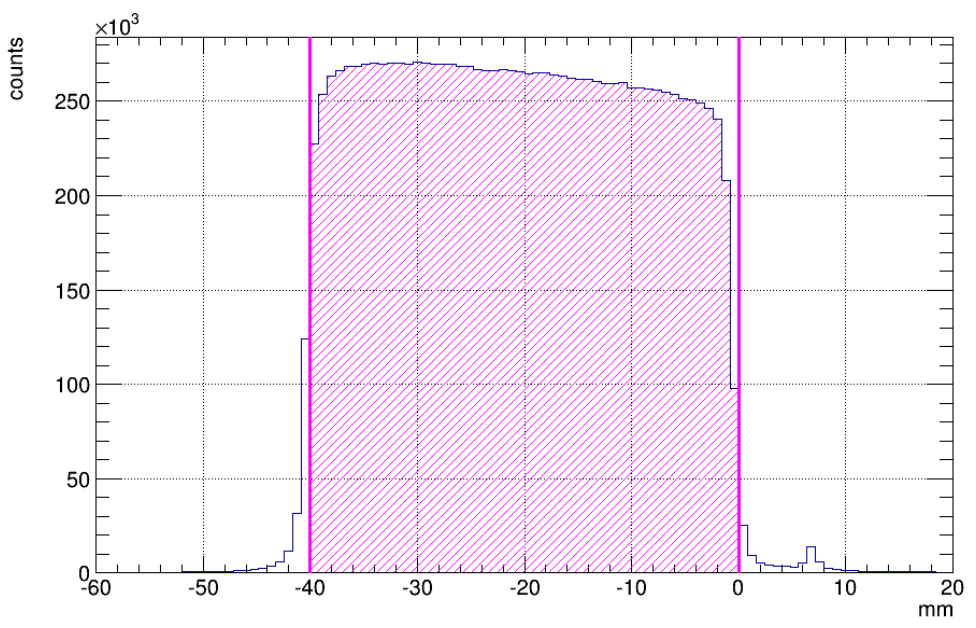

(a)

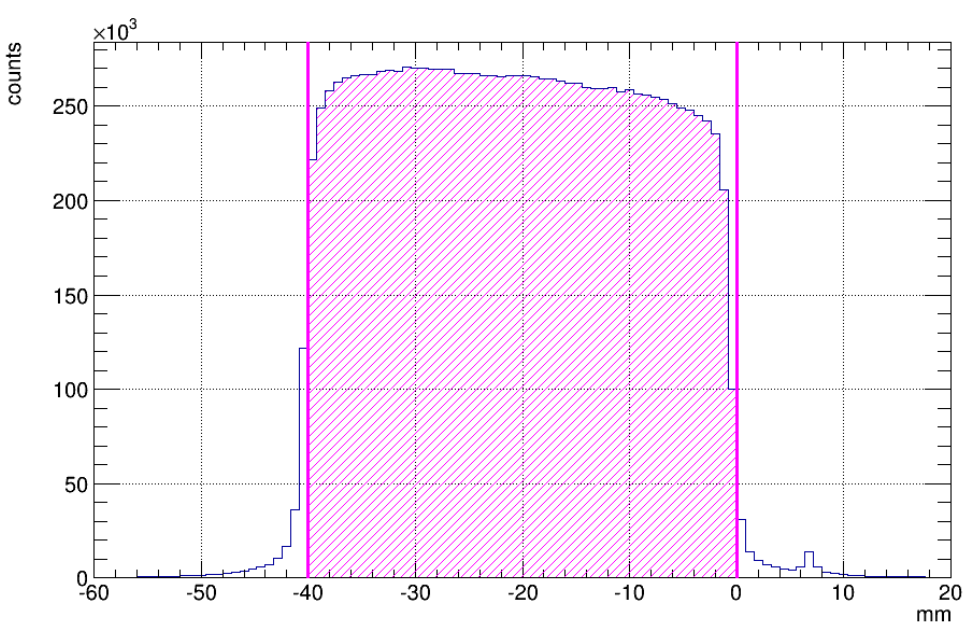

(b)

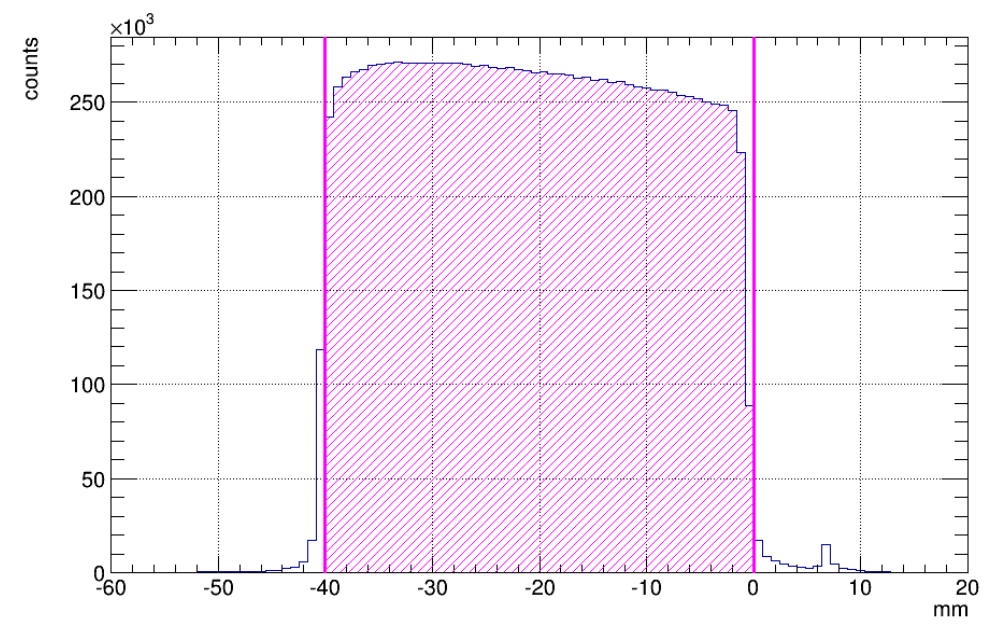

(c)

Figure 6.5: The z-vertex distributions for the proton (a), positive pion (b), and the negative pion (c) with the upper and lower limits of the cut shown with magenta lines. 


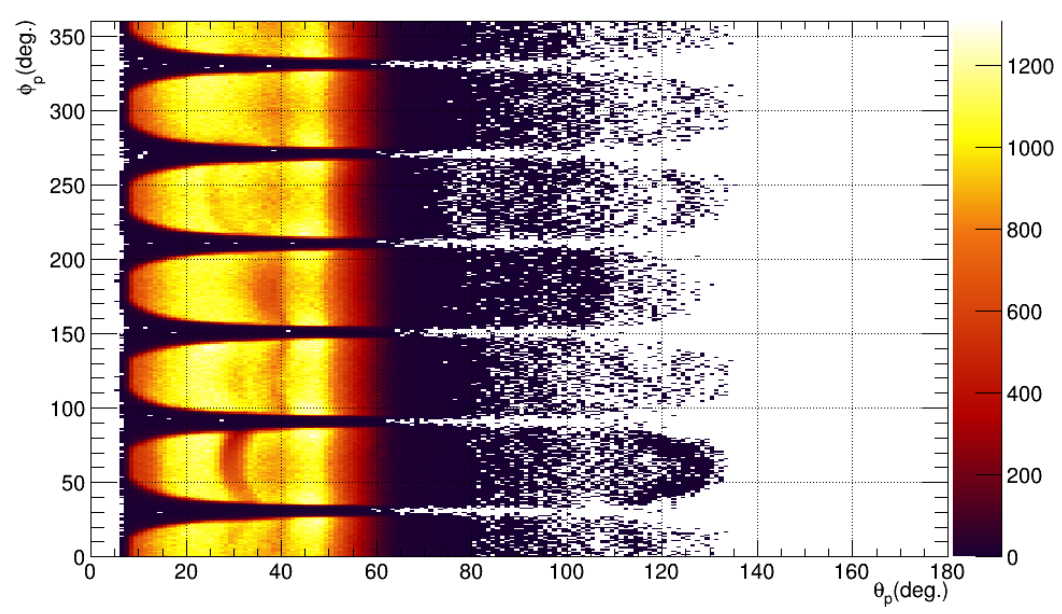

(a)

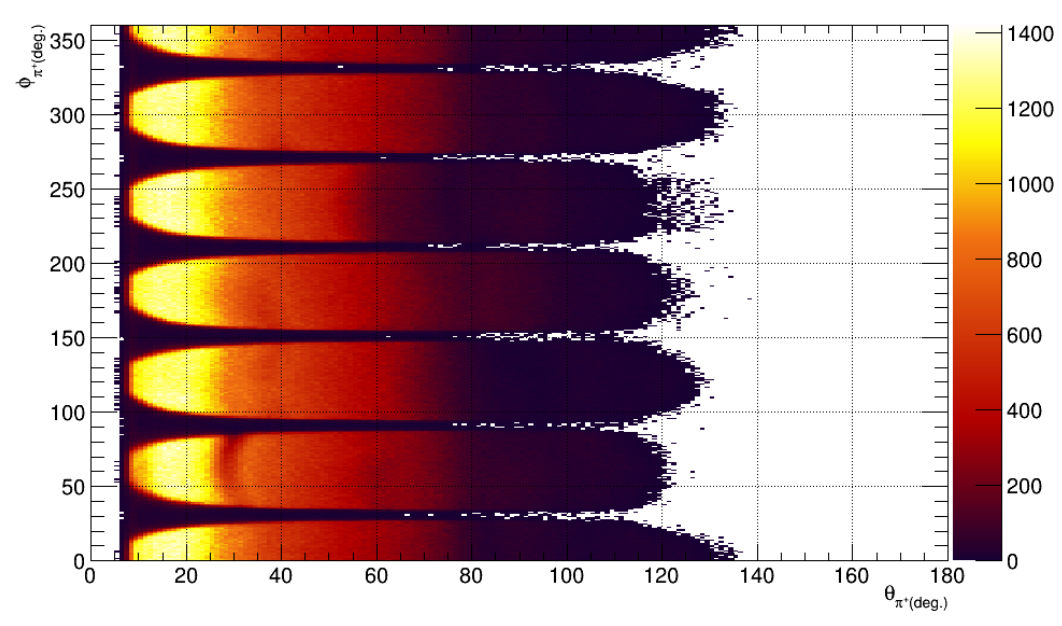

(b)

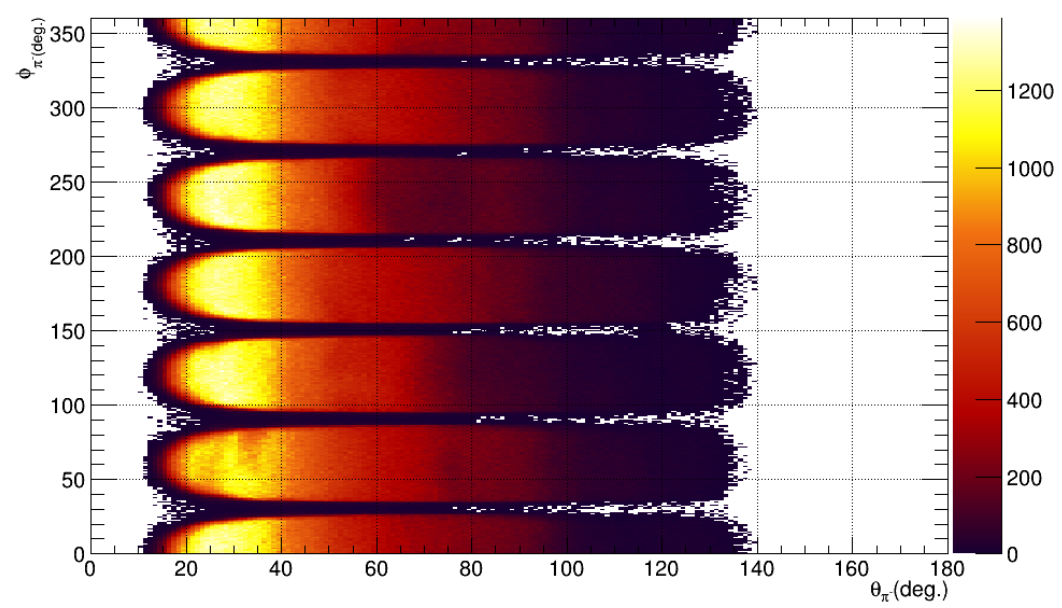

(c)

Figure 6.6: The angular distribution for the proton (a), positive pion (b), and the negative pion (c) showing the fiducial regions of the CLAS detector. 


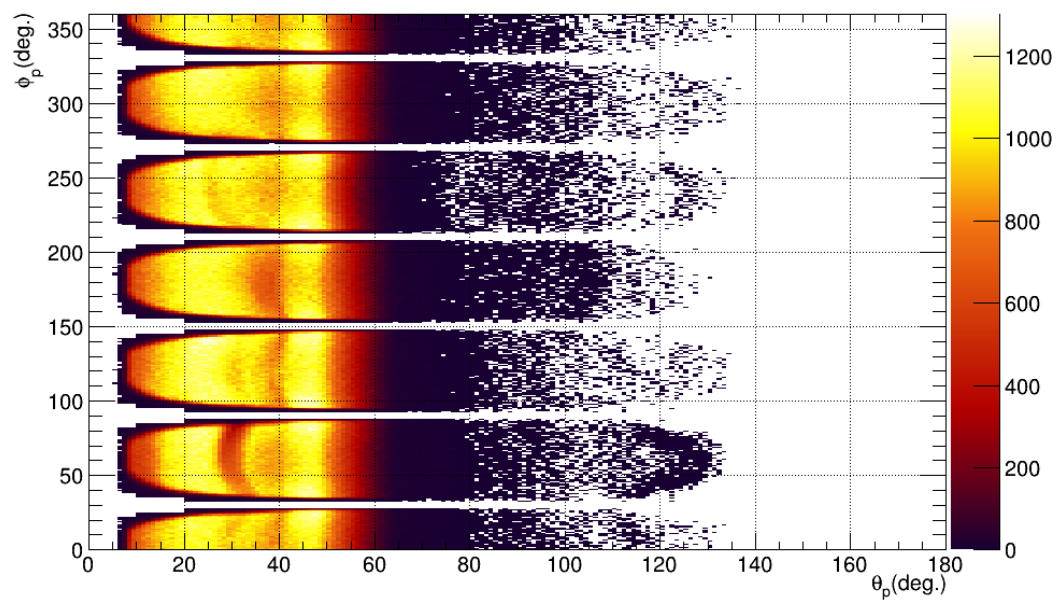

(a)

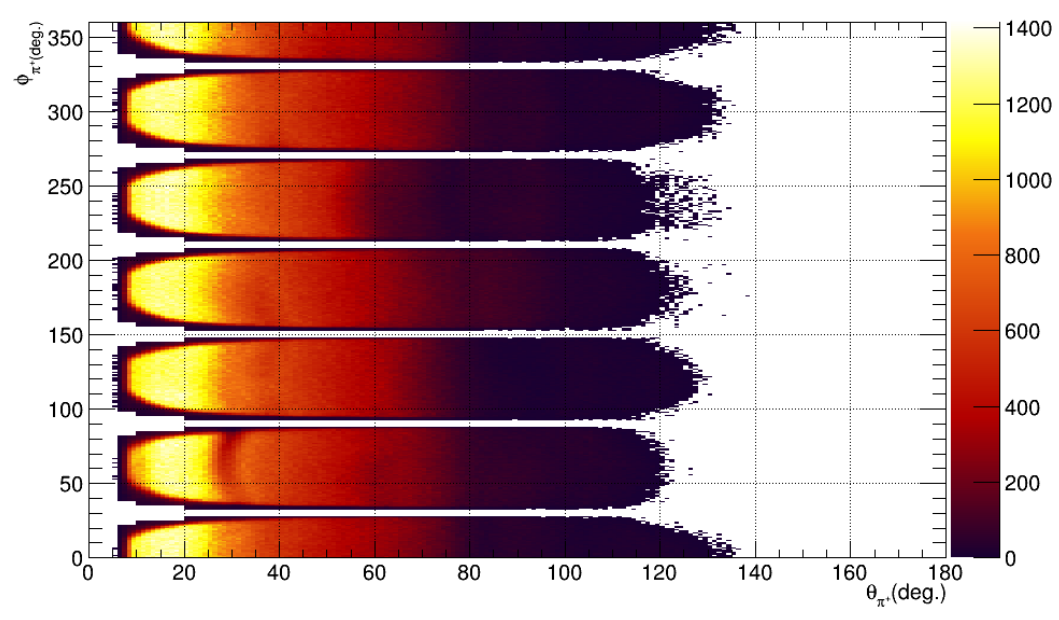

(b)

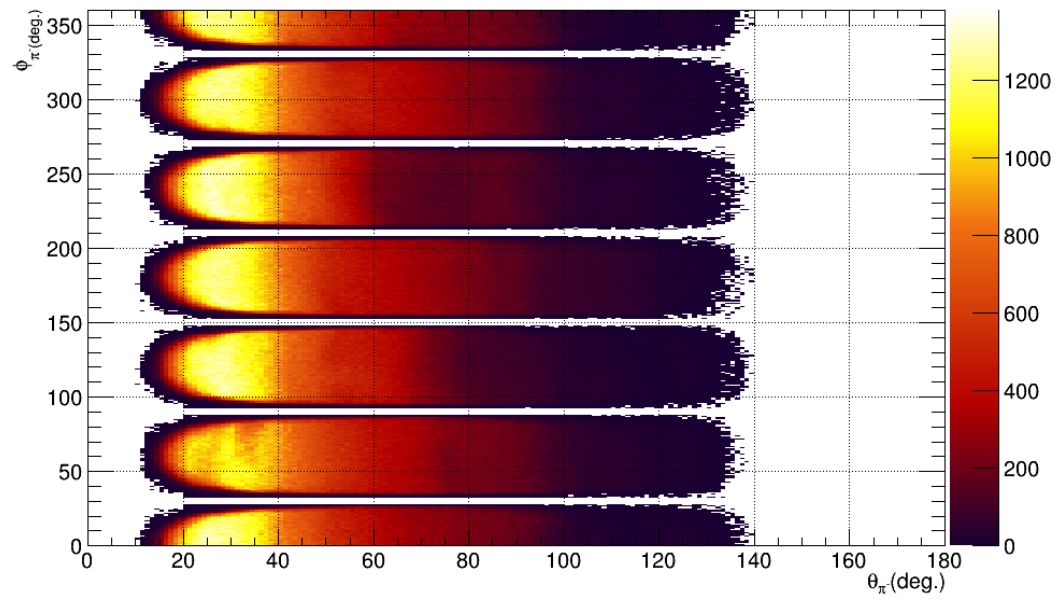

(c)

Figure 6.7: The angular distribution with the fiducial cuts applied for the proton (a), positive pion (b), and the negative pion (c). 


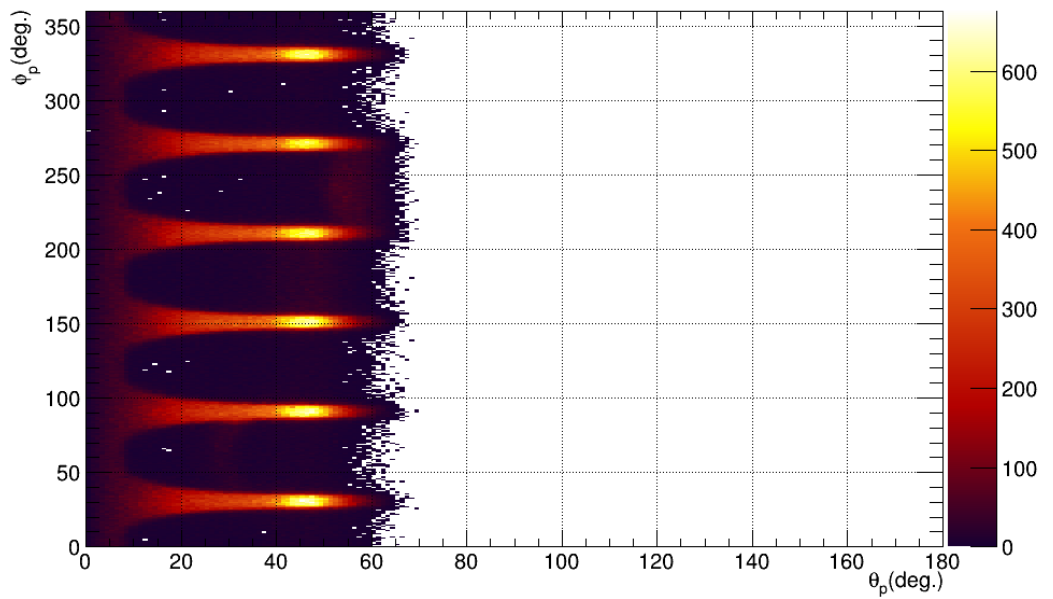

(a)

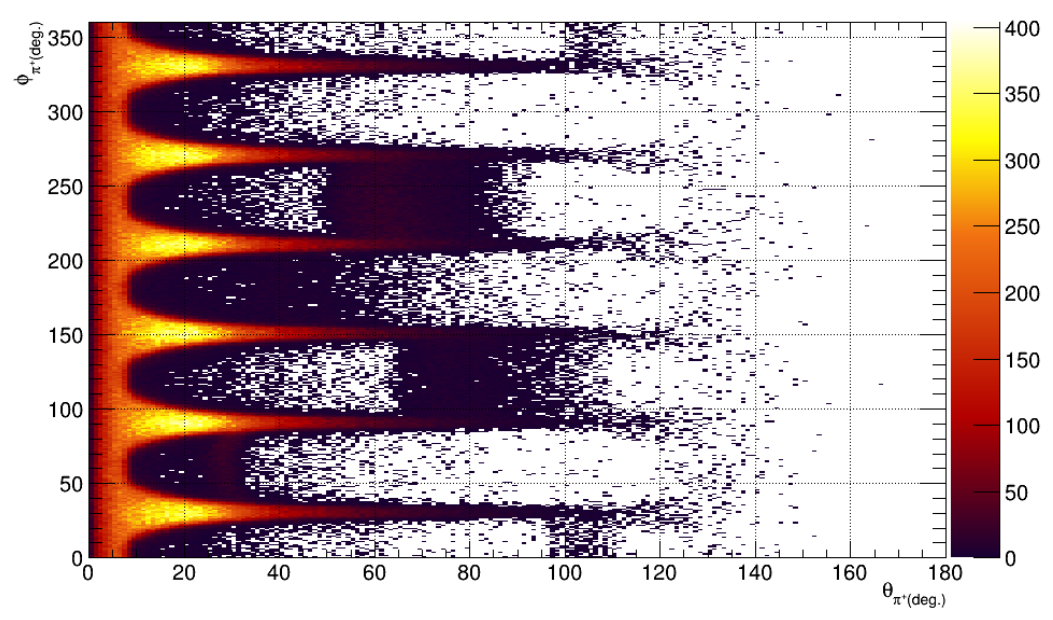

(b)

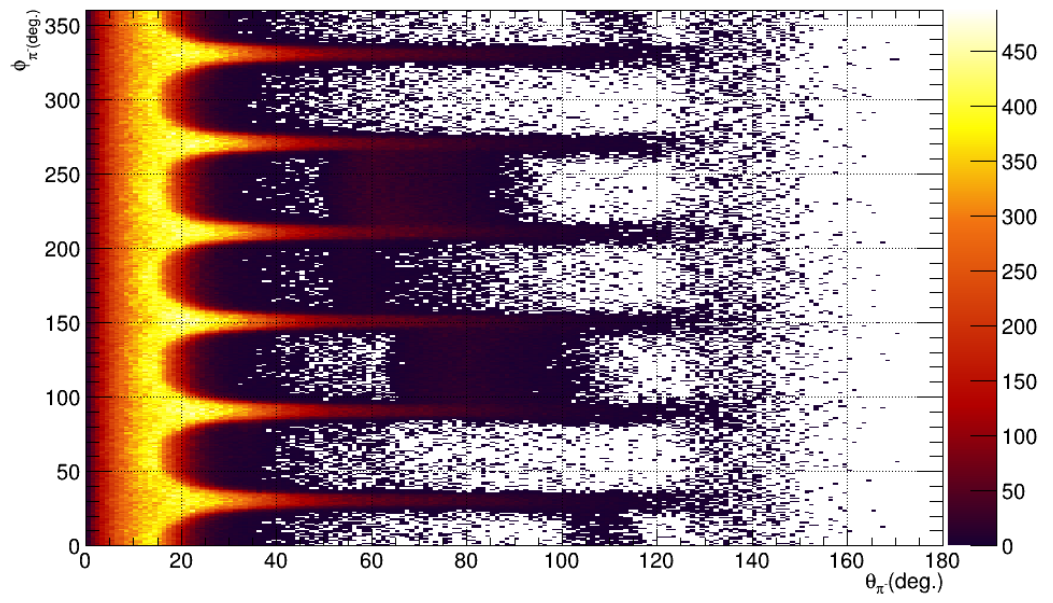

(c)

Figure 6.8: The angular distribution for the reconstructed missing particles for the proton (a), positive pion (b), and the negative pion (c). 


\subsubsection{Photon Selection}

This procedure is for pairing hadronic processes with the coincident photon. As there are potentially many more detected photons (the multiplicity for each event for the dataset can be seen in figure 6.9 due to the nature of the tagging system than there are hadronic tracks we need to decide which photon is most likely associated with a given hadronic track.

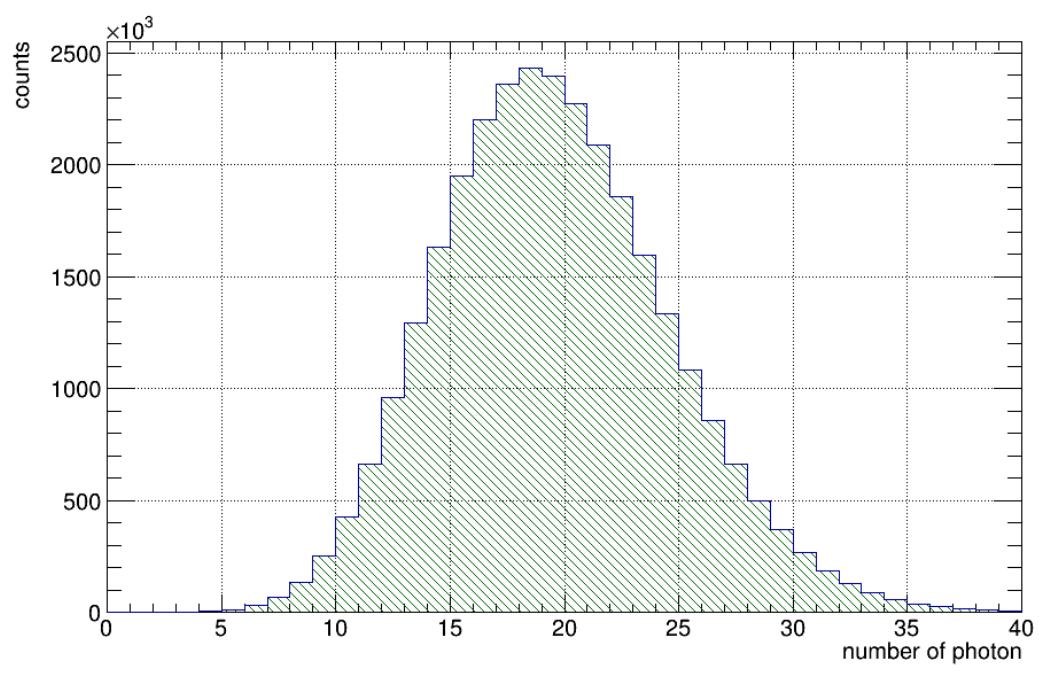

Figure 6.9: The multiplicity of photons per event. Typically there are about $15-20$ photons to choose from for each event.

First the hadronic vertex time is extropolated from the timing information from the TOF for the detected particles. This gives us the timing of the event. This time is then compared with the timing of the tagger system. The difference should be close to zero if both systems are accurately calibrated. However, the the fact that some hadrons are miscorrelated with random photons leads to a 2 ns beam bucket structure show in 6.10 . So the most likely prompt photon has to be chosen for each event. This is done by minimising the difference between the proton vertex time and the photon time, $\Delta t$ :

$$
\Delta t=T O F_{t}(p)-\frac{T O F_{p a t h}(p)}{c \beta_{p}}-\gamma_{t}+\frac{z_{p}}{c}
$$




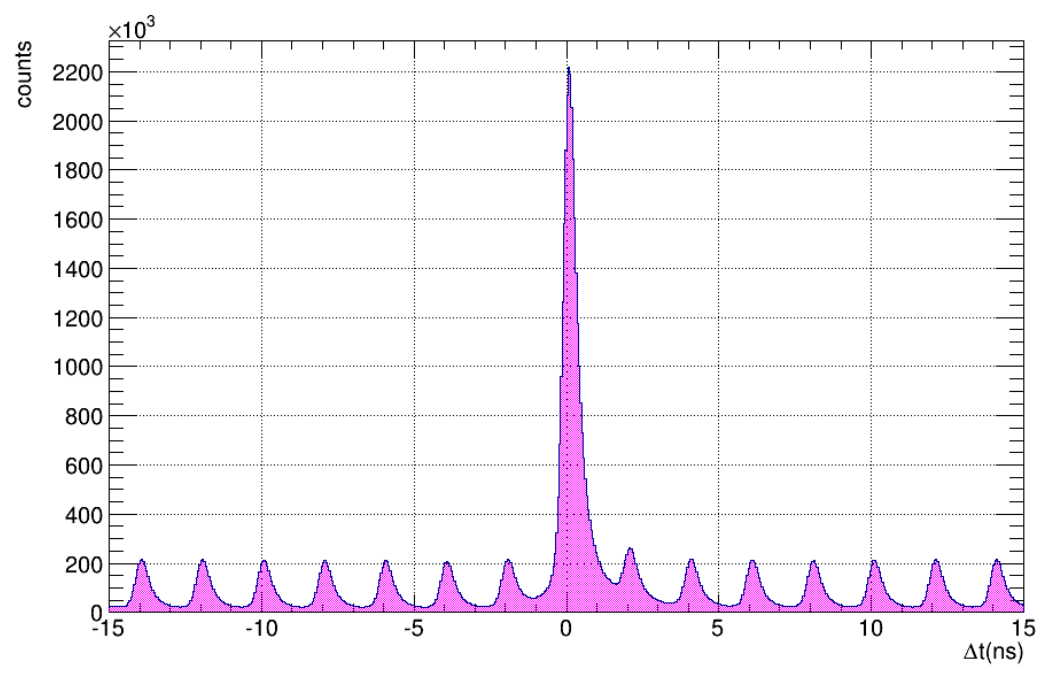

Figure 6.10: Timing difference between the hadronic TOF timing and the associated photon's timing from the tagging system. The majority are around 0ns is expected. The other 2 ns structures are due to hadrons being correalated with random photons.

Where:

$$
\beta_{p}=\frac{k_{p}}{\sqrt{k_{p}^{2}+m_{p}^{2}}}
$$

The mass of the proton used is taken from the Particle Data Group [REF] and where:

- $T O F_{t}(p)=$ proton TOF hit time

- $T O F_{\text {path }}(p)=$ proton path length from the event vertex to the TOF

- $\gamma_{t}=$ event photon vertex time to centre of the target

- $z_{p}=\mathrm{z}$-vertex position of the proton

- $c=$ speed of light

The result of this procedure is shown in 6.11

For the case of the event topology where the proton is missing the positive pion is used for the best photon procedure. Figure 6.12 shows the timing before the procedure where the same beam bucket structure is apparent, and figure 6.13 shows the timing after the procedure. 


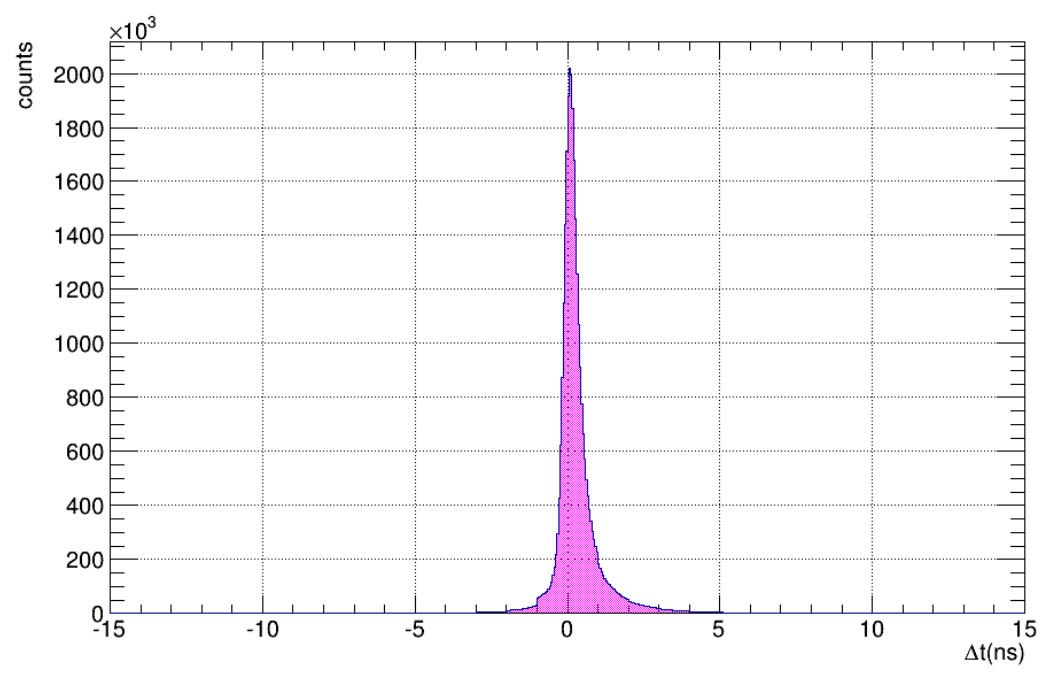

Figure 6.11: Timing difference between the hadronic TOF timing and the associated photon's timing from the tagging system after the best photon procedure. The majority are around $0 \mathrm{~ns}$ is expected. The asymmetric nature of the distributions is due to the proton vertex time being momentum dependent. The correction for this is described in section 6.1.7.

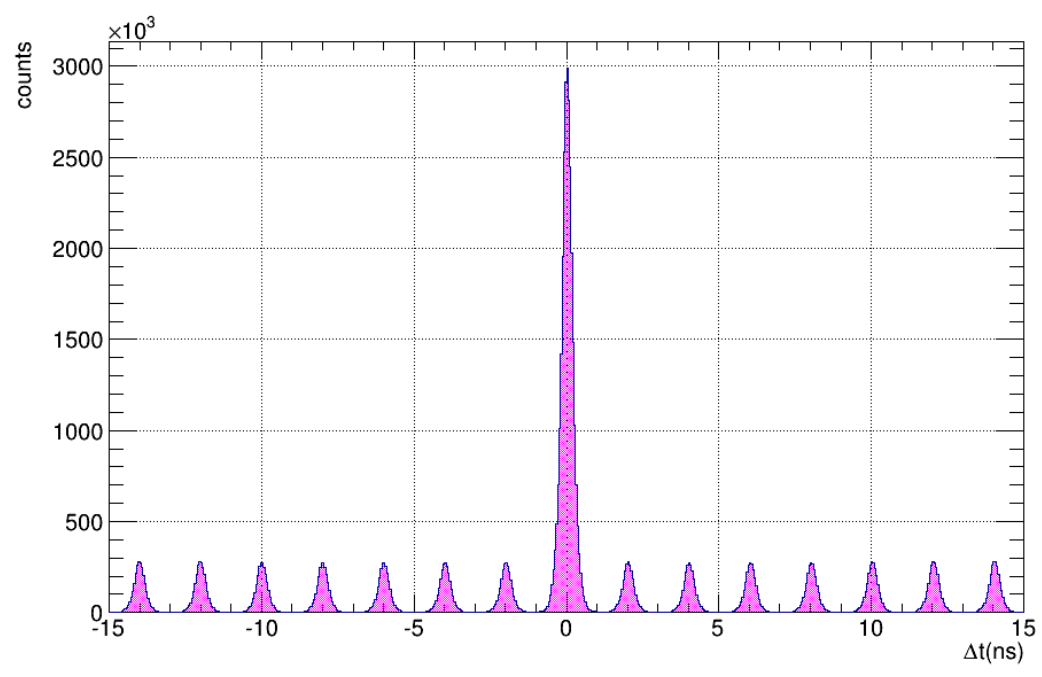

Figure 6.12: Timing difference between the positive pion TOF timing and the associated photon's timing from the tagging system for the events with a missing proton. The majority are around $0 \mathrm{~ns}$ is expected. The other $2 \mathrm{~ns}$ structures are due to hadrons being correalated with random photons. 


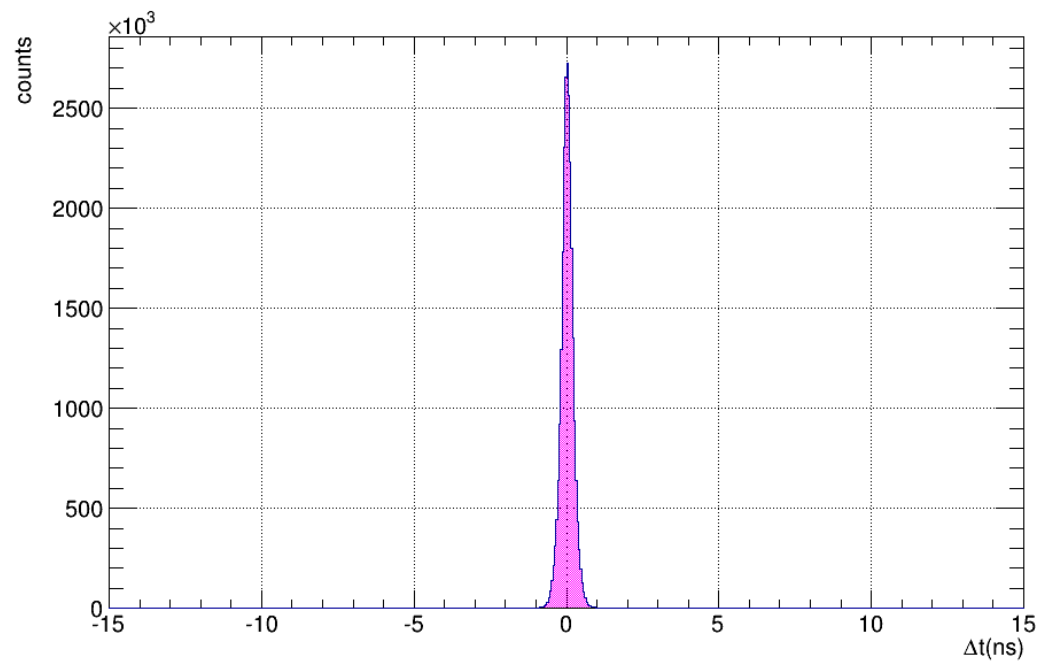

Figure 6.13: Timing difference between the positive pion TOF timing and the associated photon's timing from the tagging system after the best photon procedure for the events with a missing proton. The majority are around 0ns is expected.

\subsubsection{Tagger Correction}

As mentioned in 4.3 one of the corrections needed for the photon tagging system was due to the large magnet sagging over time. It therefore changed its geometry and so the original energies taken from tagger channels would be incorrect. This results in a measured energy for the electrons different from their actual energy and subsequently the corresponding tagged photons. So tagger channel specific coefficients are calibrated and applied to the best photon at this stage [115].

\subsubsection{Momentum Dependent Timing Cuts}

This first momentum cut is applied to all charged particles. A minimum threshold of $300 \mathrm{MeV} / \mathrm{c}$ is used. This corresponds to the minimum detection momentum of CLAS.

After this momentum dependent timing cuts are implemented. This dependency is due to the slowing of particles in the target region and the drift chambers before they reach the TOF region. The higher the mass the more effect this as so the detected protons are the affected the most in this analysis. Figure 6.14 shows the momentum dependency of all of the particles before any cuts are applied. A series of fits are 


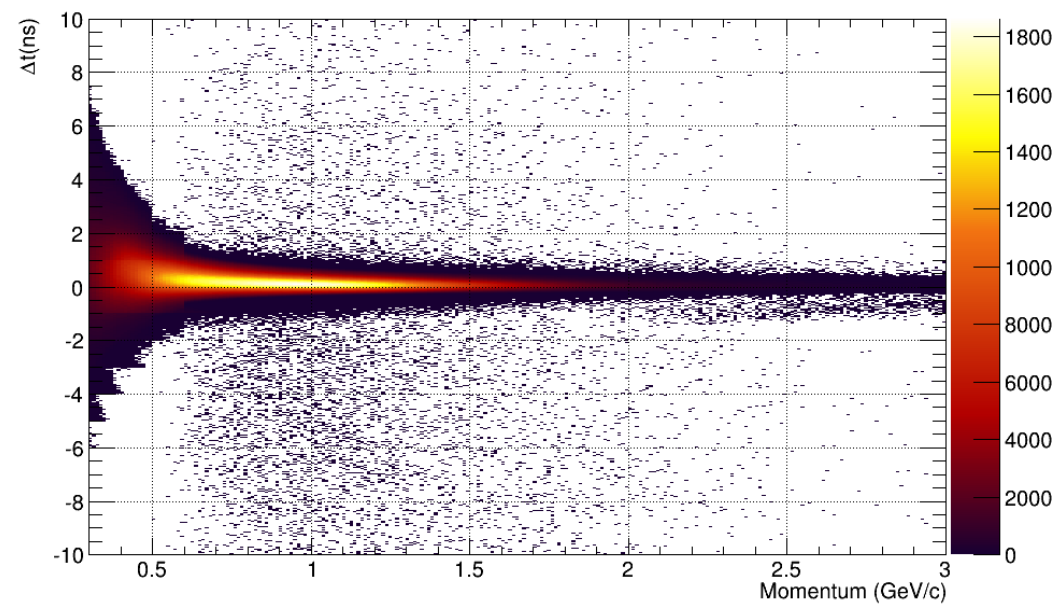

(a)

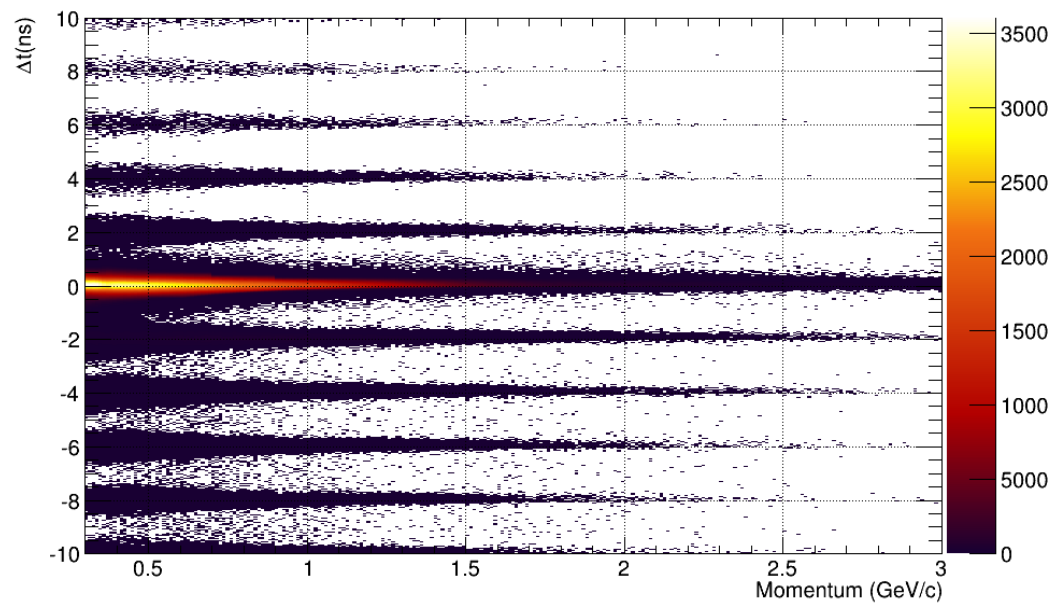

(b)

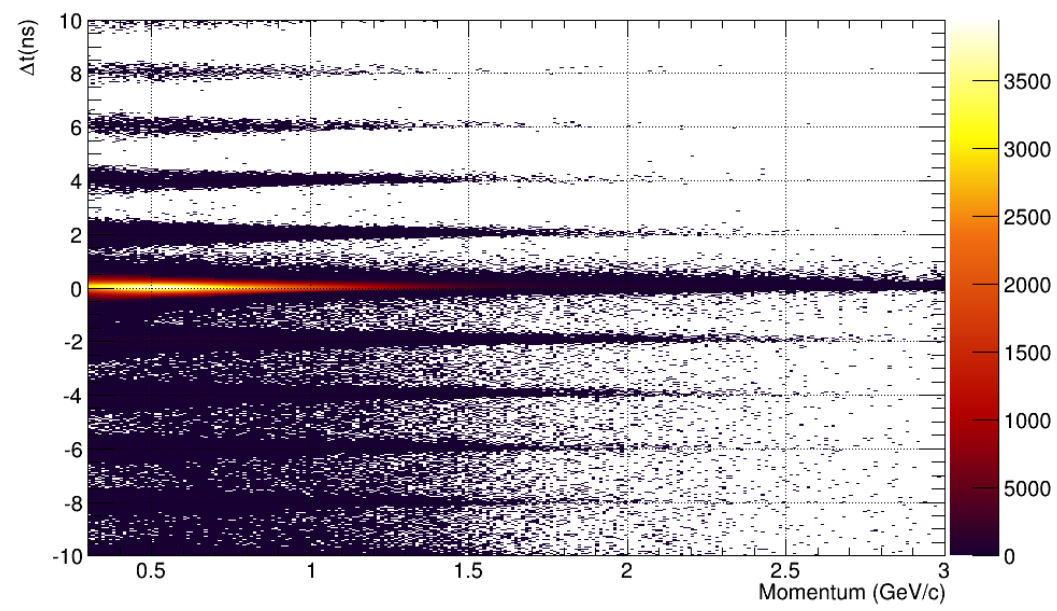

(c)

Figure 6.14: The momentum of the final state particles as a function of the difference between their timing vertex and the corresponding incident photon's tagger timing for the proton (a), positive pion (b), and the negative pion (c). 
applied in $100 \mathrm{MeV} / \mathrm{c}$ bins. These are simply guassian distribution on a first degree polynomial. From these fits a $\pm 3 \sigma$ cut is applied in each bin. The effects of these cuts are show in 6.15

\subsubsection{Energy Loss Correction}

After the coincident correalation is fully implemented the energy of the particles lost in the target and the surrounding material needed to be corrected. This was done using the ELOSS (Energy Loss) package developed for the CLAS detector [116].

The ELOSS package takes the 4-vector of a detected or reconstructed particle and passes it through algorithms in order to find each path length of the particlein the separate detector regions. Then it uses these pathlengths and the characteristics of the material from a given region and determines the momentum of the particles at the reaction vertex. This process can be used for any charged particle heavier than an electron.

The energy loss calculated for the detected particles can be seen in figure 6.16 . Also, the energy loss is shown as a function of momentum in figure 6.17. It is clear that this is a greater problem for more massive particles with low momentum.

\subsubsection{Reaction Channel Angle Cut}

In this section a simple cut is applied to the angle between the proton and reconstructed particle from which the pions decayed. The angle should be $\approx 180^{\circ}$ and so a stringent cut is applied at $177.0^{\circ}$. This is shown in figure 6.18 .

\subsubsection{Missing Mass}

The missing mass of the two particles, e.g. for a detected proton and a positive pion, is reconstructed. If this reconstructed particle has a mass which falls within $\pm 3 \sigma$ of that of a pion, it is attributed to the missing pion, in this example the negative one. Due 


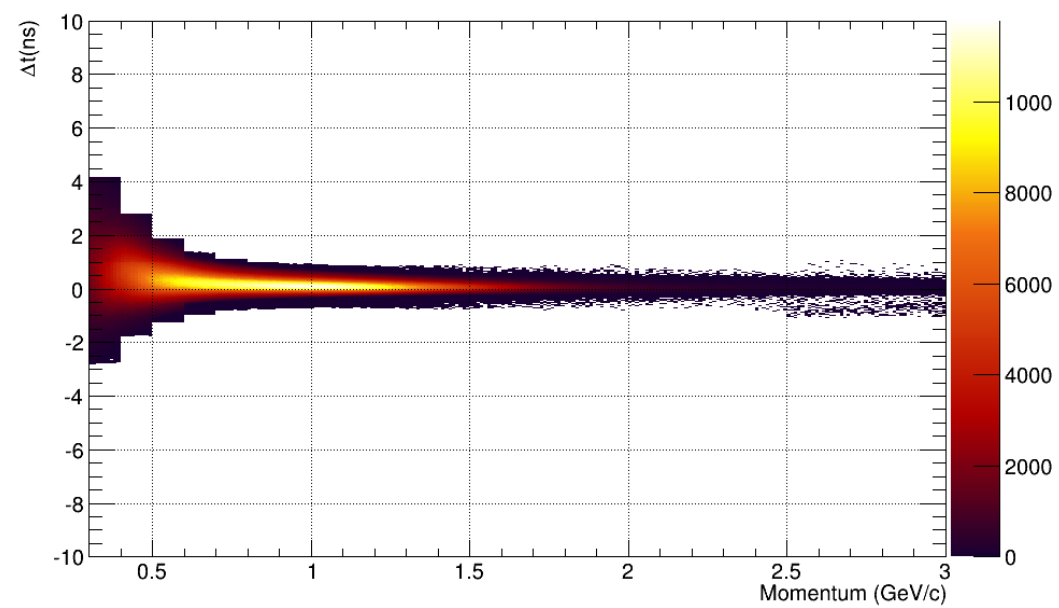

(a)

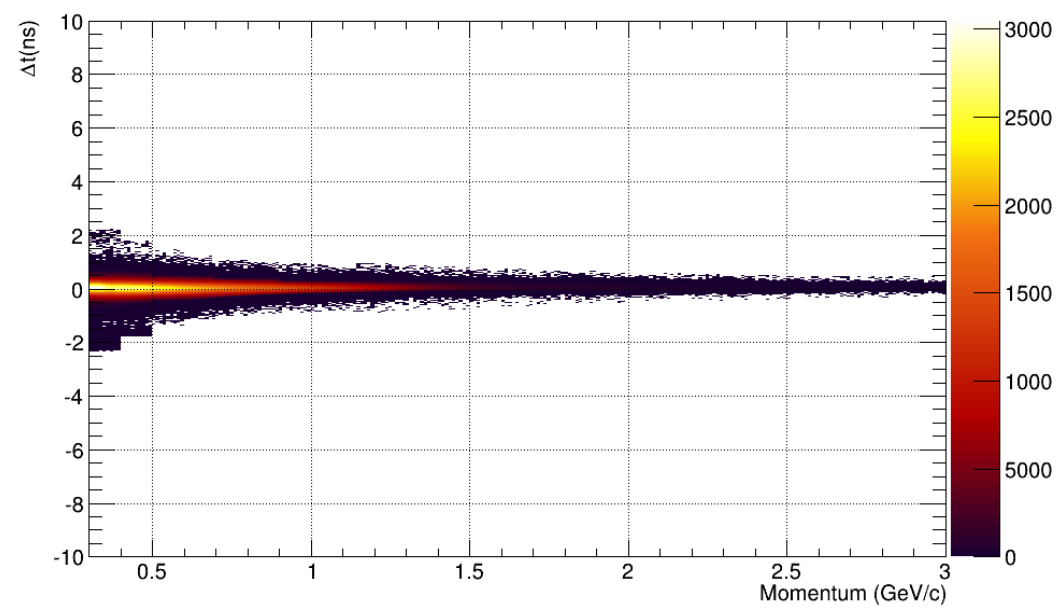

(b)

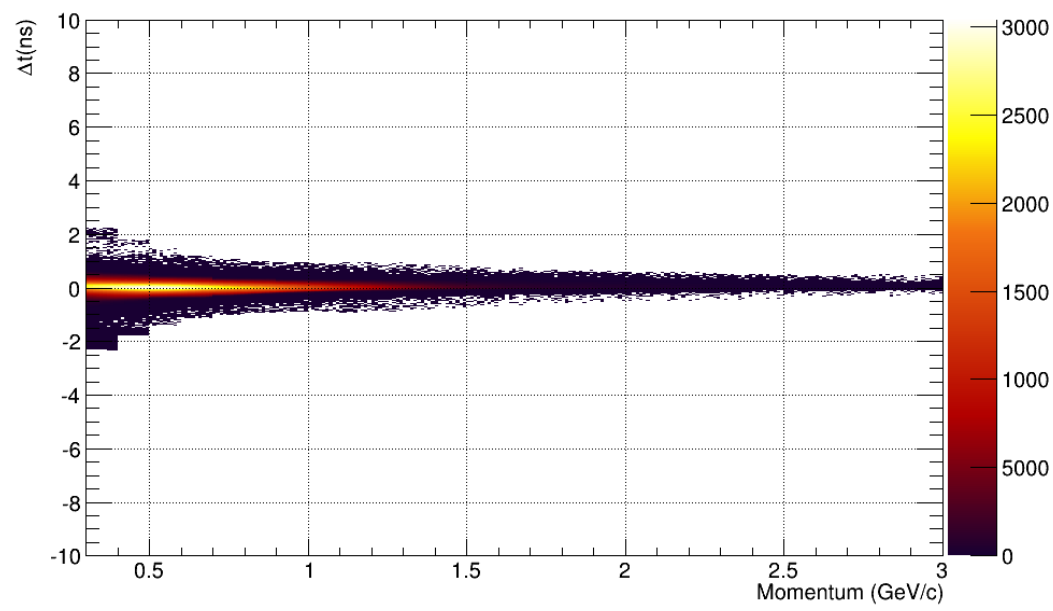

(c)

Figure 6.15: The momentum of the final state particles as a function of the difference between their timing vertex and the corresponding incident photon's tagger timing with the cuts applied as described above for the proton (a), positive pion (b), and the negative pion (c). 


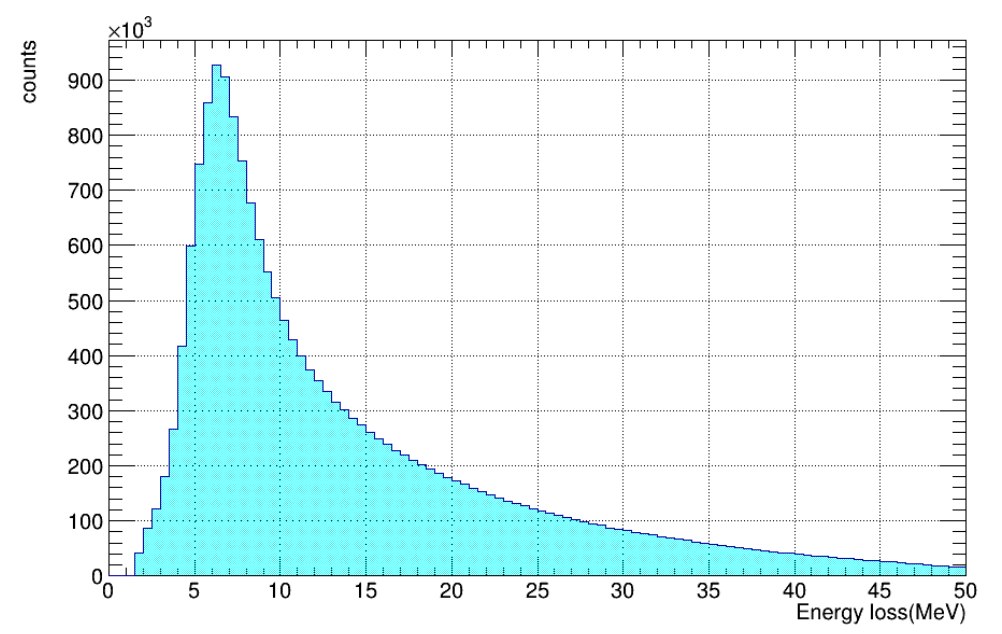

(a)

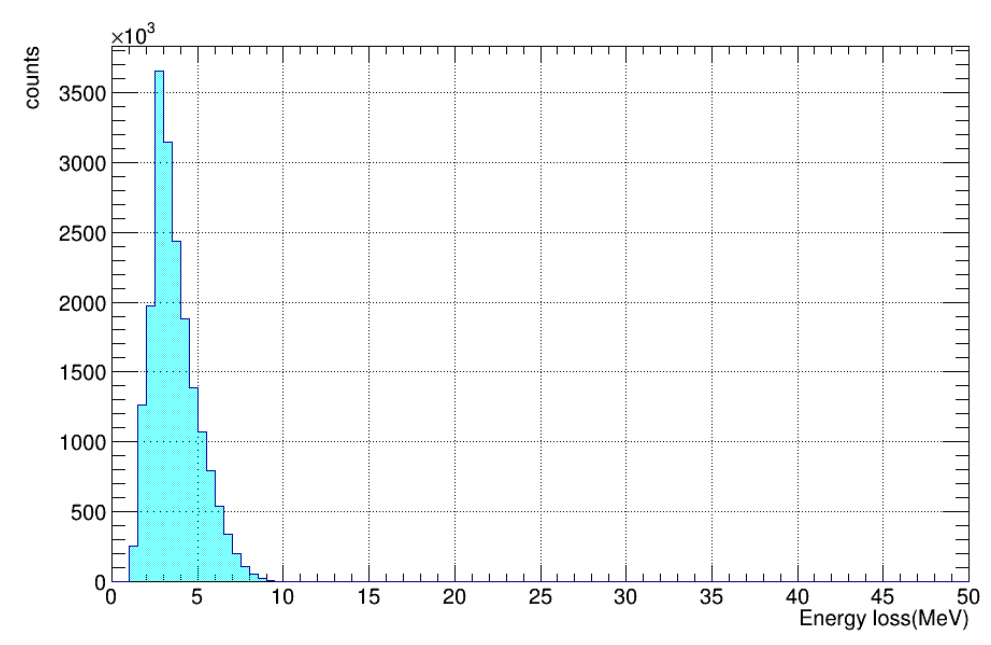

(b)

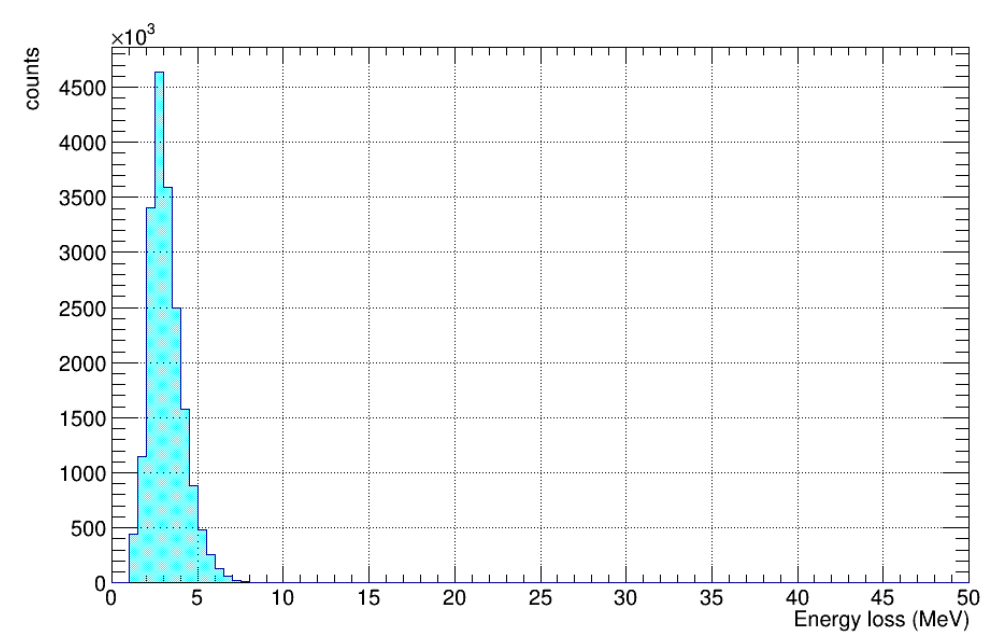

(c)

Figure 6.16: The energy loss calculated using the ELOSS package for the proton (a), positive pion (b), and the negative pion (c). 


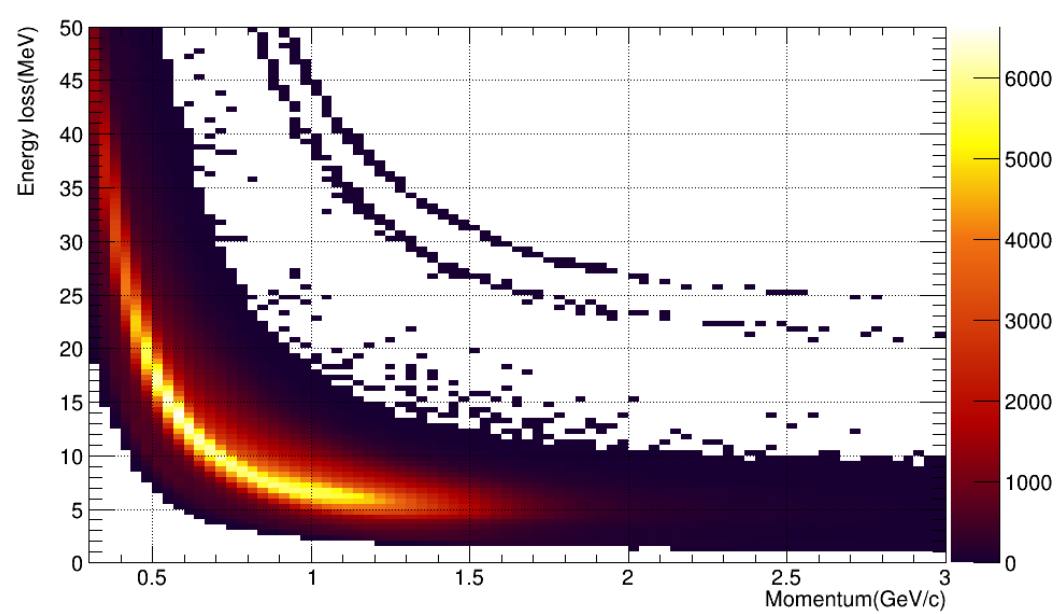

(a)

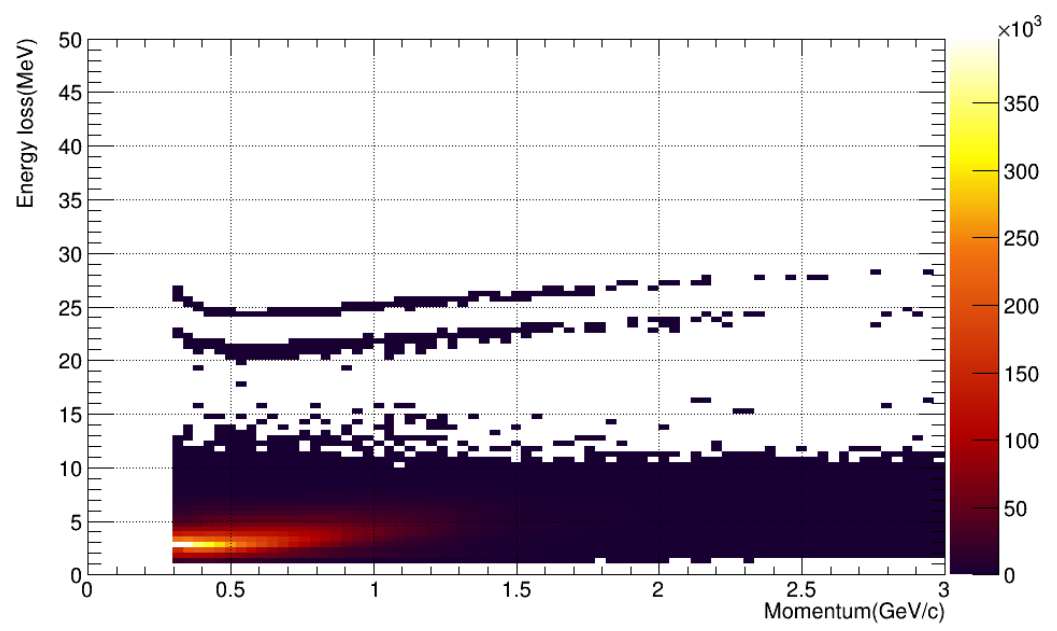

(b)

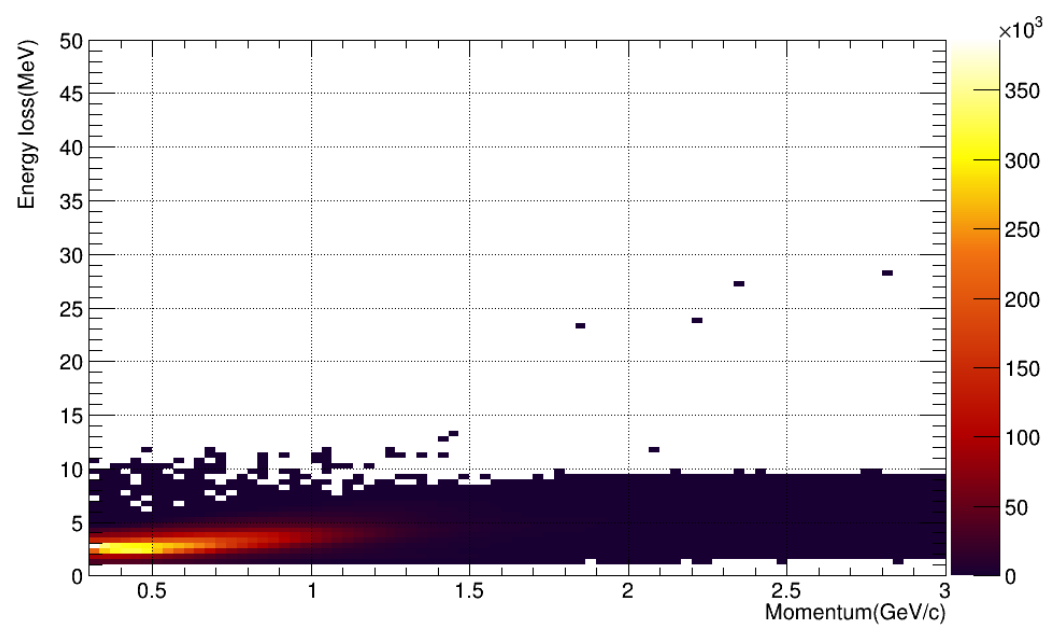

(c)

Figure 6.17: The energy loss calculated using the ELOSS package as a function of momentum for the proton (a), positive pion (b), and the negative pion (c). 


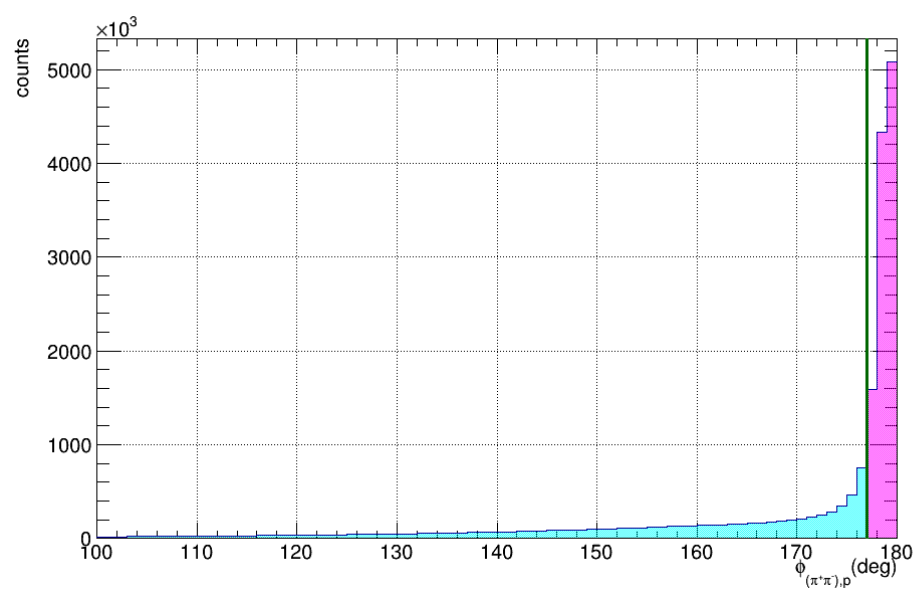

Figure 6.18: The angle between the proton and the reconstructed particle from which the pions decayed. The green line shows the cut at $177.0^{\circ}$ and the magenta highlighted area shows the acceptance regiona.

to charge conservation, if we presume that there is no other particle missing, then the missing pion must be of the opposite charge of the detected one. The missing mass plots of the three different topologies used are shown in figure 6.19, figure 6.20, and figure 6.21 with the accepted regions for each highlighted in magenta.

At this stage a similar process was applied to the reconstructed missing particles as in section 6.1.2 in order to apply more restrictive limits on the missing particles than just on their mass. The $\Delta \beta$ versus momentum plots are shown in figure 6.23 for before the cuts and in figure 6.24 for after the cuts. Similarly, figure 6.22 shows the beta versus momentum distributions before the cuts and figure 6.25 shows the effect of the cuts.

From this stage these reconstructed particles are considered to the respective missing particle for its topology and the analysis of the spin observables is carried out using all four topologies. 


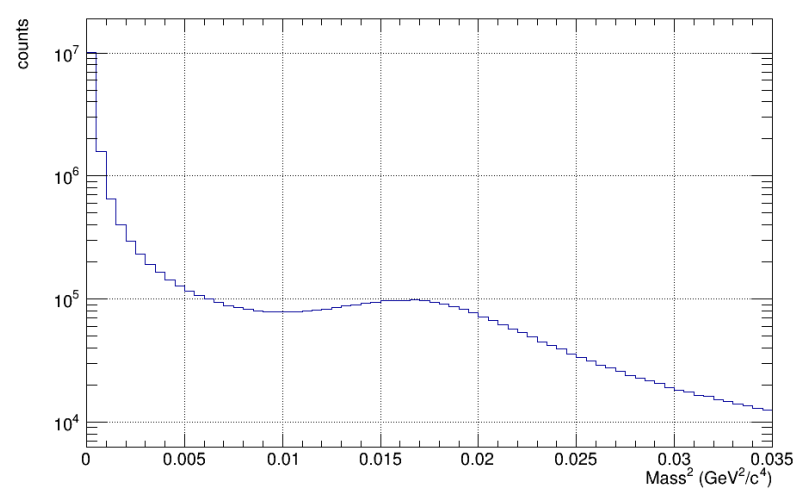

(a)

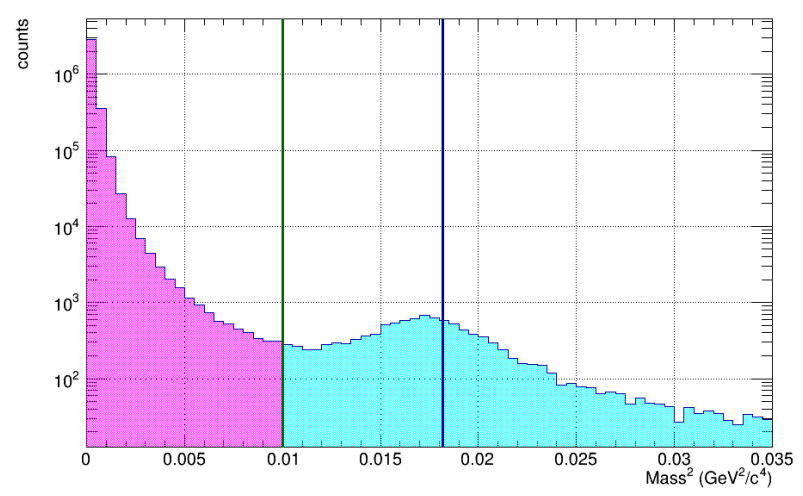

(b)

Figure 6.19: The missing mass squared of the exclusive topology. a) shows the missing mass squared before the cut on the angle between the two pions and the proton in the reaction plane and b) shows it afterwards with the regions of acceptance shown in magenta with green line showing the edge of the accepted region. The events which have a missing mass in the region shown in cyan were dismissed. The blue line shows the position where the mass peak of an undetected neutral pion would lie. 


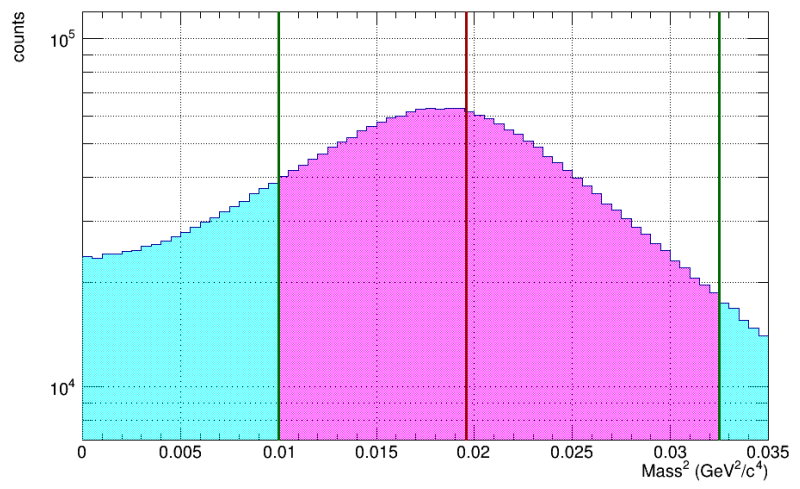

(a)

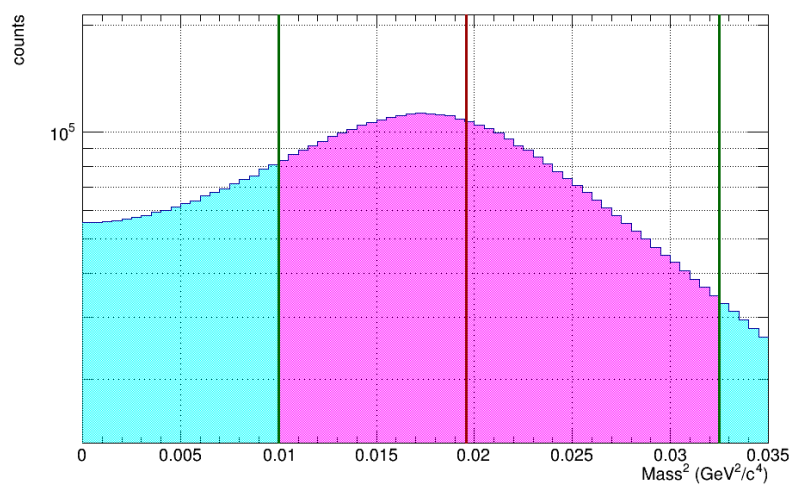

(b)

Figure 6.20: The missing mass squared distributions for the case of the undetected pion: first the positive pion in a) and then the negative pion in b). The red line highlights the theoretical postion of the charged pions mass squared, the green lines the upper and lower limits of the cuts which are the same used for the detected particles and stated in table 6.1, the magenta highlighted area is the area of acceptance and the cyan highlighted area is the area of dismissal. 


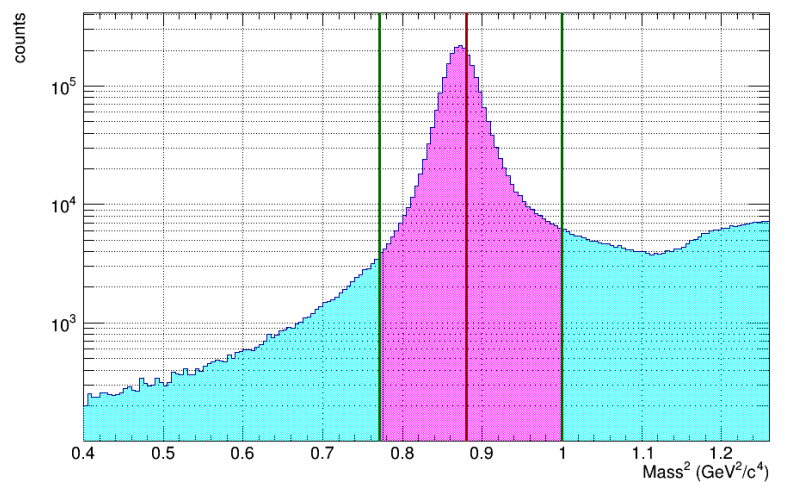

Figure 6.21: The missing mass squared distributions for the case of the undetected proton: first the positive pion in a) and then the negative pion in b). The red line highlights the theoretical postion of the proton mass squared, the green lines the upper and lower limits of the cuts which are the same used for the detected particles and stated in table 6.1, the magenta highlighted area is the area of acceptance and the cyan highlighted area is the area of dismissal. 


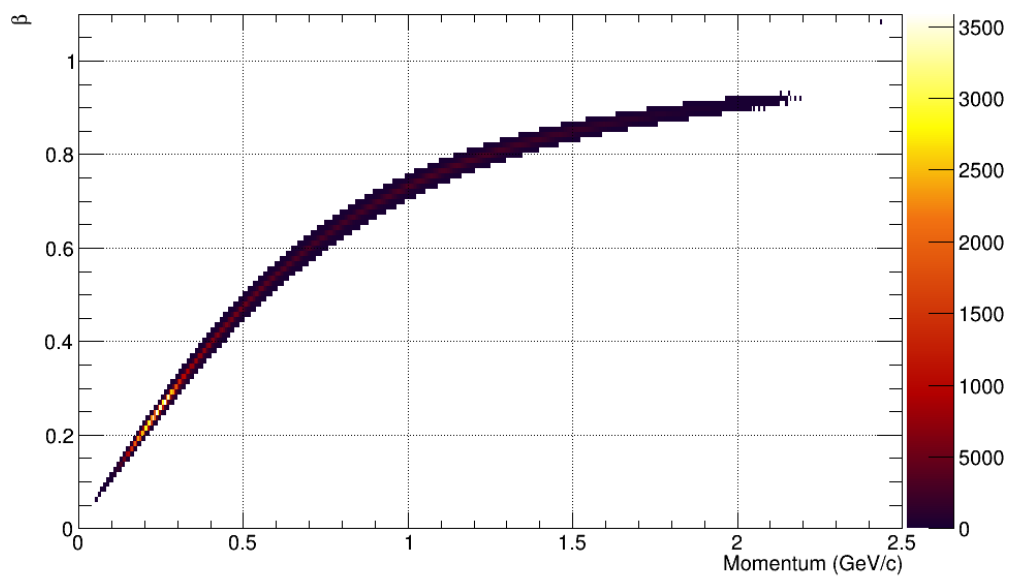

(a)

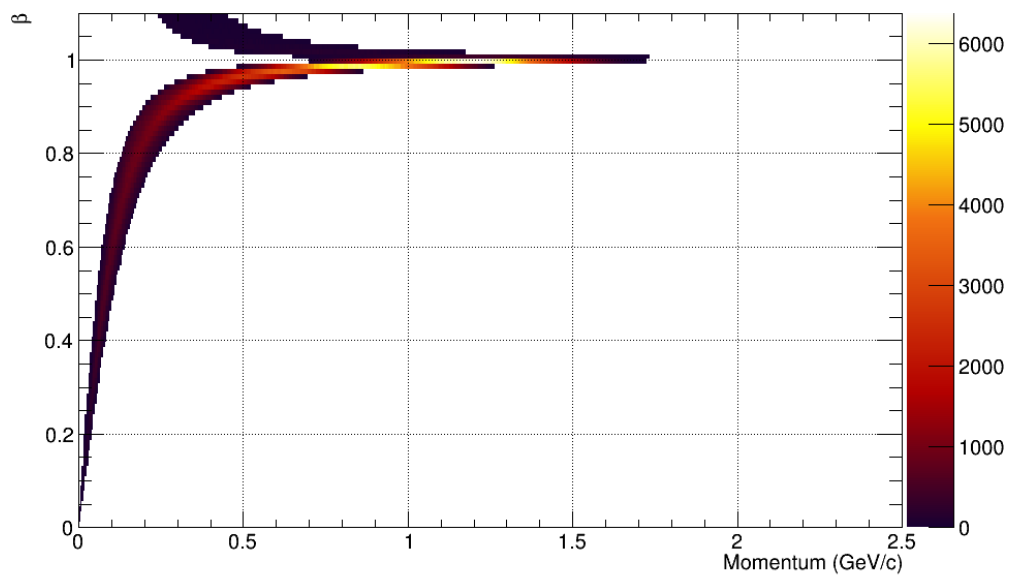

(b)

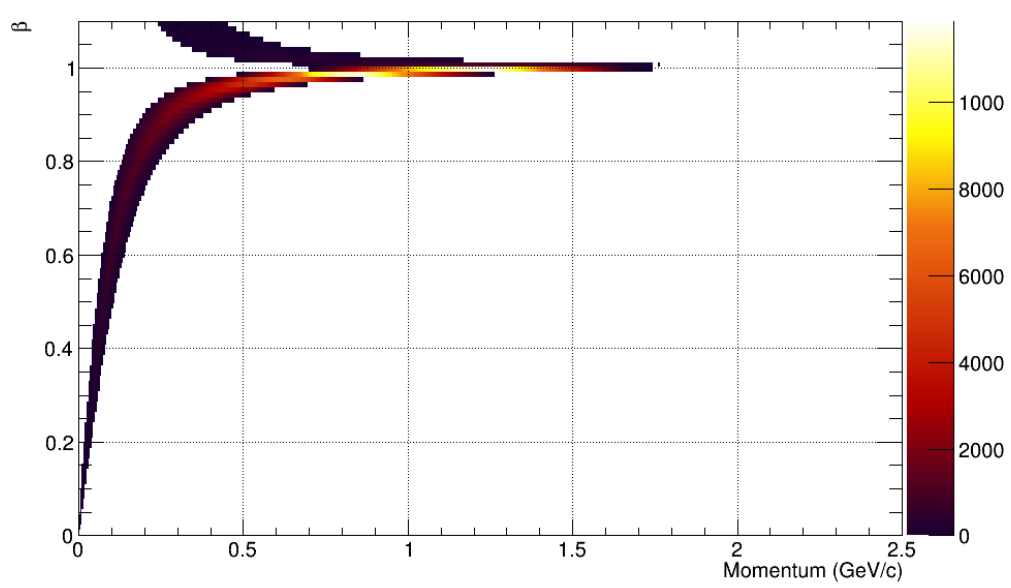

(c)

Figure 6.22: The measured beta of the reconstructed particles as a function of the momentum for a) the missing proton, b) the missing postive pion, and c) the missing negative pion. Distinct tracks can be seen corresponding to the pions and the proton (the lower beta track in the positive particle plot). 


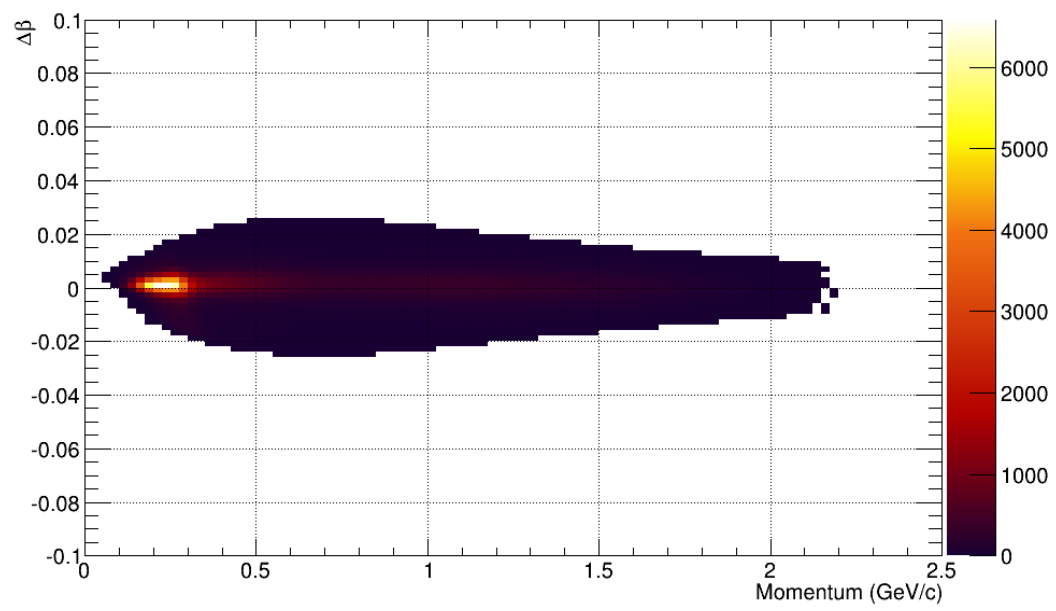

(a)

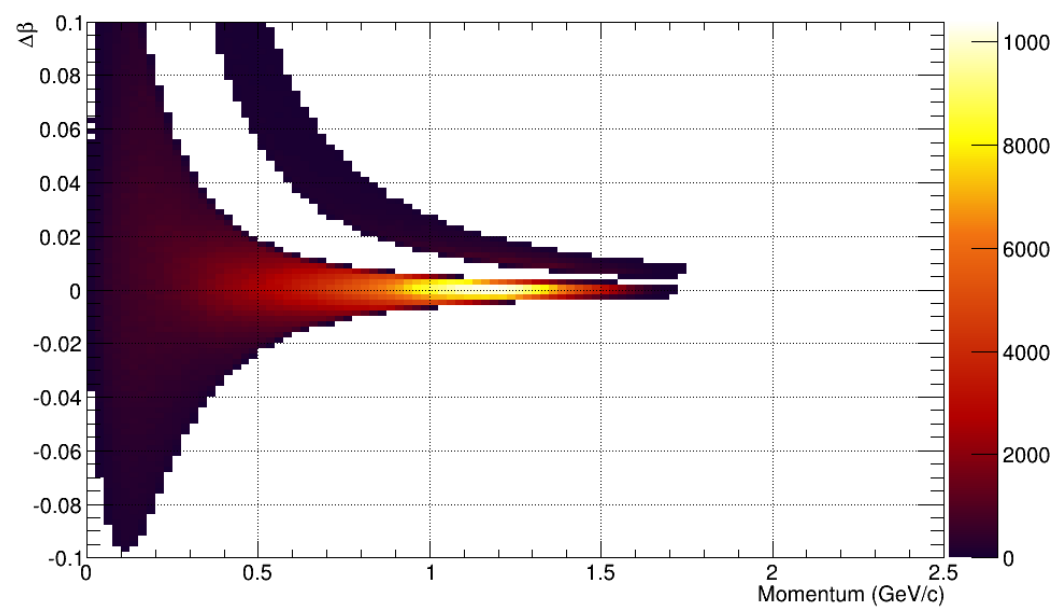

(b)

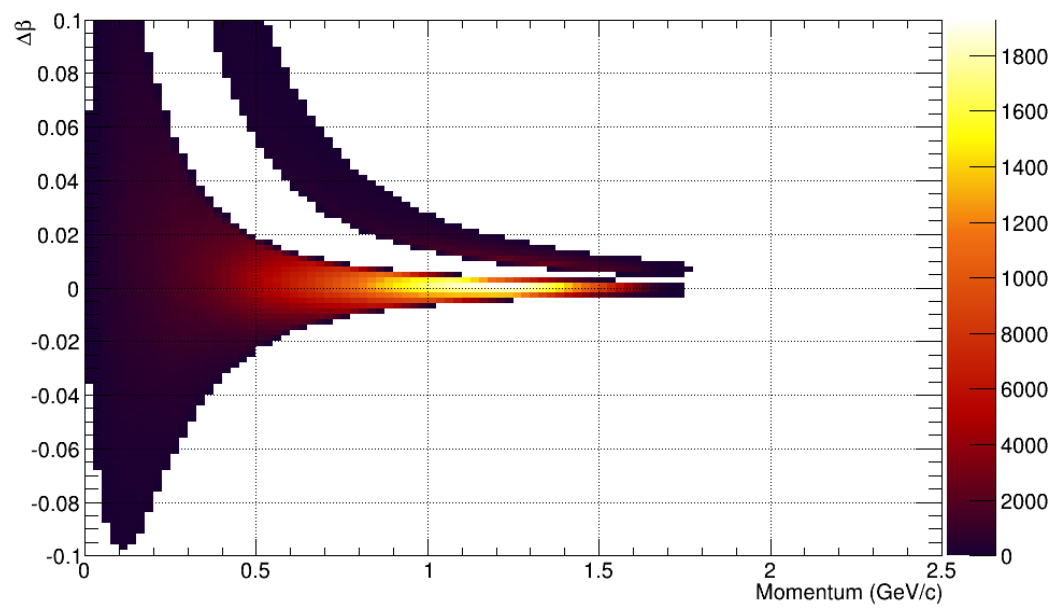

(c)

Figure 6.23: The difference between the measured beta of the reconstructed particles and the calculated value for the corresponding expected particle type as a function of the momentum for a) the proton $\mathrm{b}$ ) positive pion and c) the negative pion. 


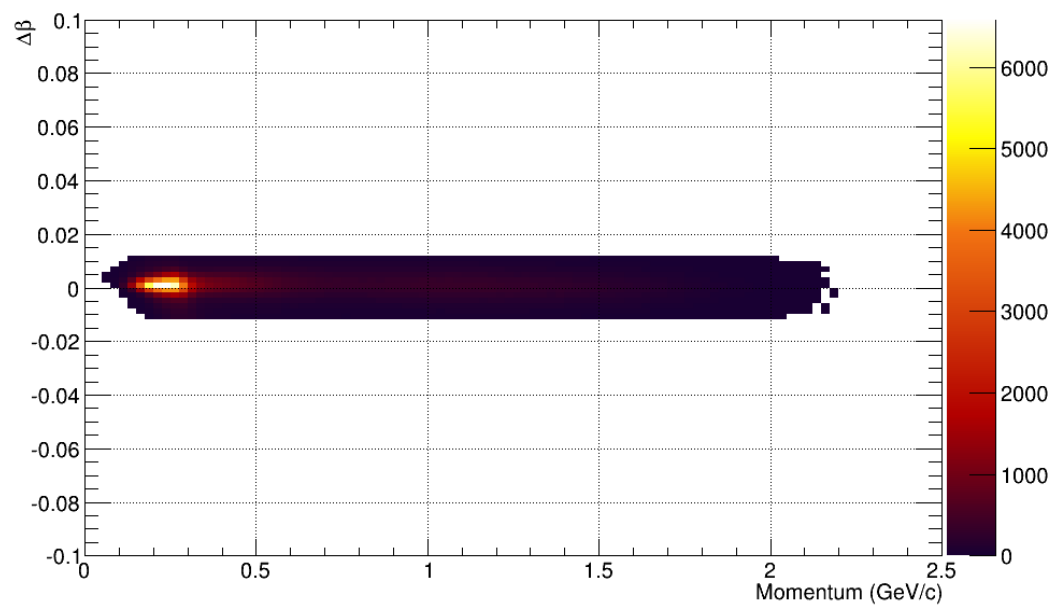

(a)

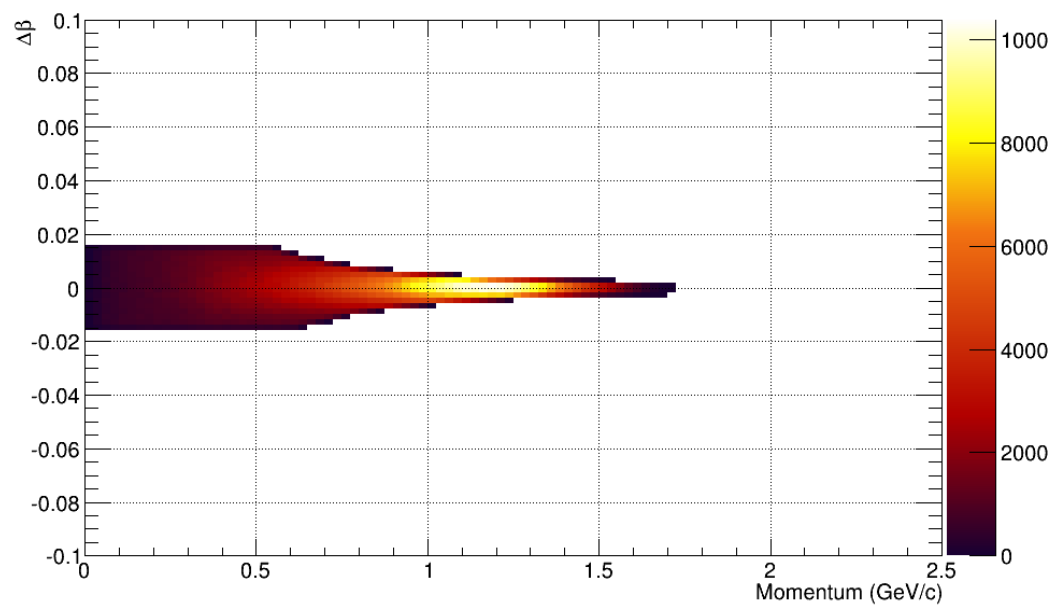

(b)

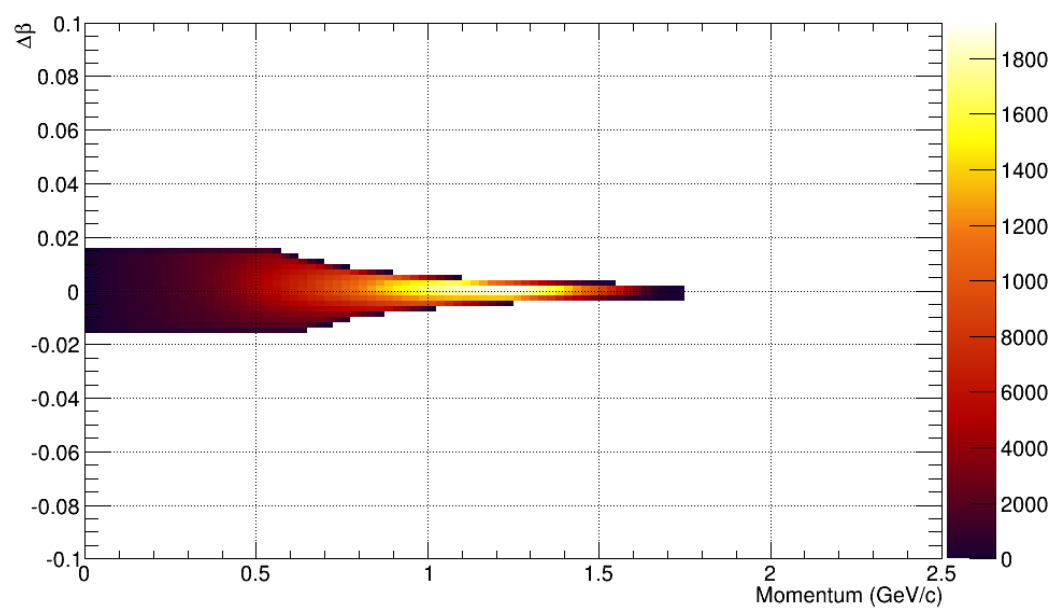

(c)

Figure 6.24: The difference between the measured beta of the reconstructed particles and the calculated value for the corresponding expected particle type as a function of the momentum with the cuts applied for a) the proton $b$ ) positive pion and c) the negative pion. 


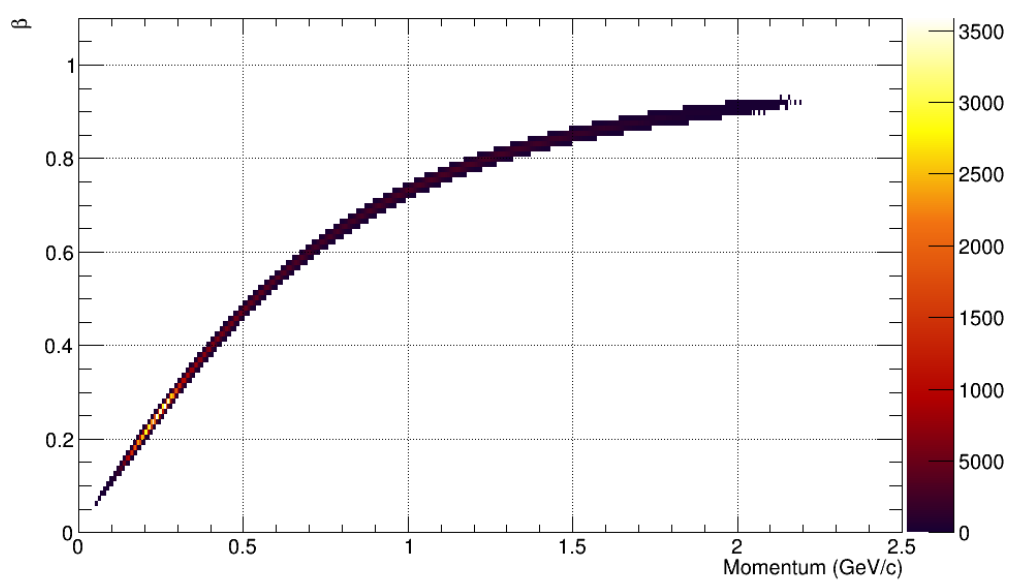

(a)

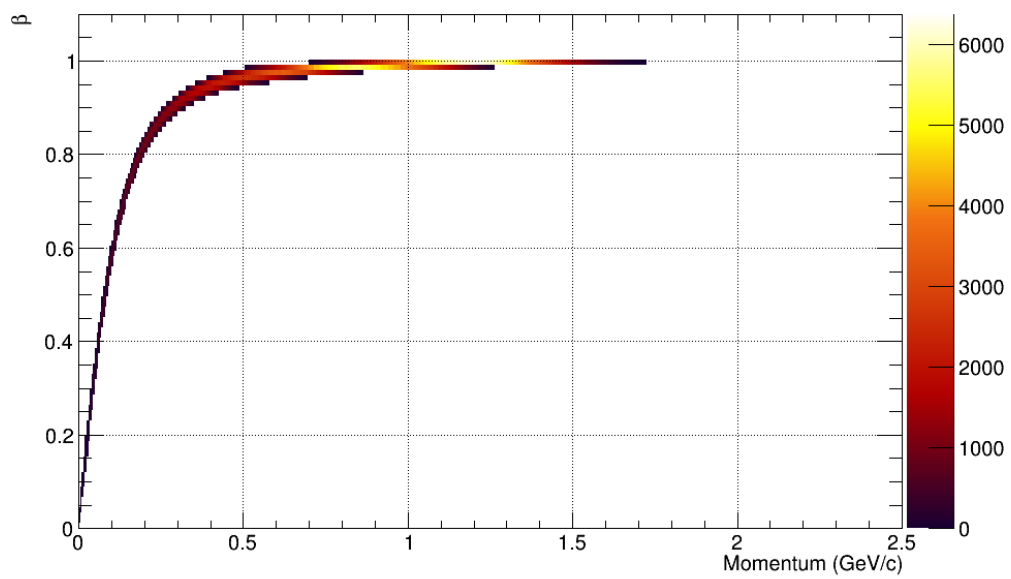

(b)

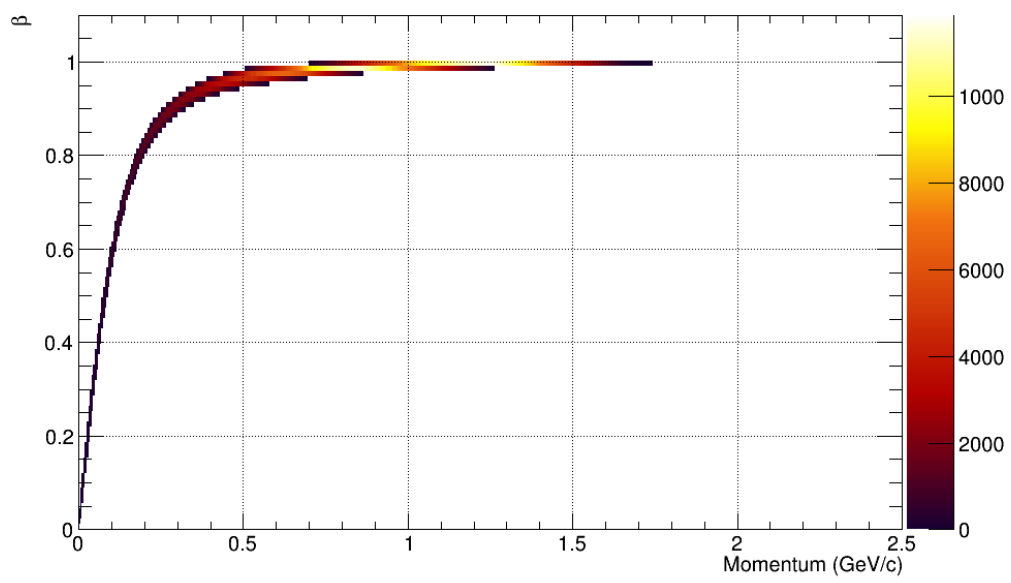

(c)

Figure 6.25: The measured beta of the reconstructed particles as a function of the momentum after the $\Delta \beta$ cuts for a) the proton, b) the postivie pion, and c) the negative pion. The majority of the background has been removed in comparison to 6.22. 


\subsection{Channel Selection}

Having selected events with the correct final state for $\rho^{0}$ photoproduction the next stage is to separate the signal of the $\rho^{0}$ meson from other processes. As can be seen in figure 6.26, in different kinematic regions the invariant mass of the two pions has a different distribution; in 6.26a the $\rho^{0}$ is seen on its own lying atop a background, in $6.26 \mathrm{~b}$ another structure accompanies the $\rho^{0}$ signal, the $f^{0}$ meson.

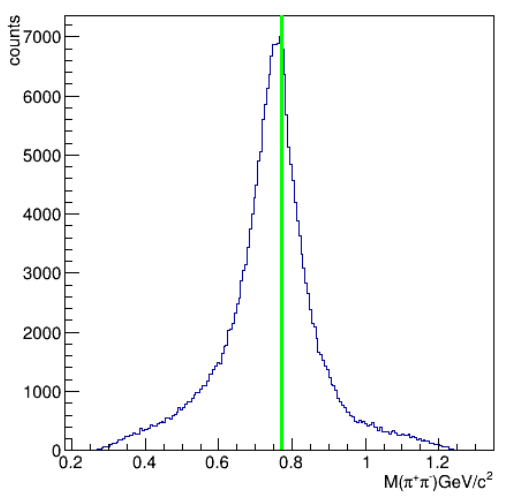

(a) The invariant mass of the two pions in the forward angle region and low W. Here the $\rho^{0}$ signal is most prominent.

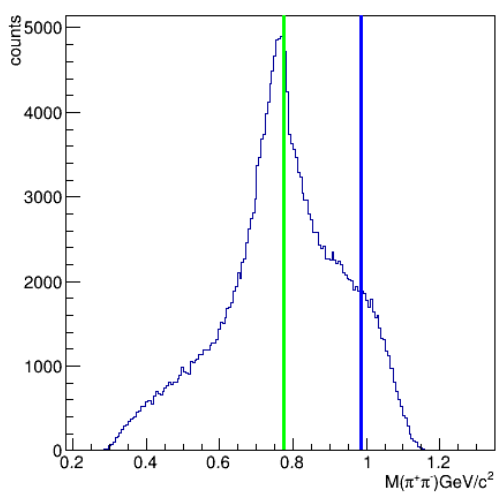

(b) The invariant mass of the two pions in the backward angle region and high $\mathrm{W}$. Here the $f^{0}$ signal can been seen in addition to the $\rho^{0}$ signal.

Figure 6.26: Invariant mass of the two pions in different kinematic regions.

Multiple channels contribute to the final detected state of $p \pi^{+} \pi^{-}$. The $\Delta^{++}$and the $\Delta^{0}$ have a strong presence, especially at lower energies. This can be seen in figure 6.27. Other known hadronic resonances become more apparent at higher energies such as $N(1520)$ and $N(1685)$ [117][118][51]. These contributions can be seen in 6.28. In addition to the complexity of studying a channel with many entangled processes, the $\rho^{0}$ meson has a large width which covers the background contributions.

The standard technique used to separate photoproduced meson signals and background processes when analysing polarization observables is to apply a phenomenological fit to the invariant mass spectra of the decay products of the meson of interest. An example of this is shown in figure 6.29 for a similar analysis of photoproduced 


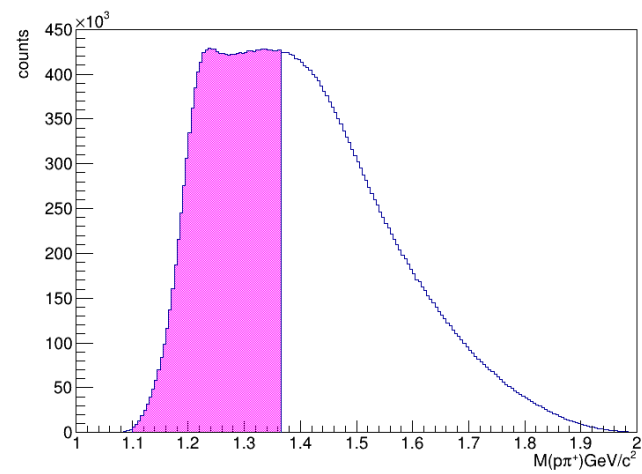

(a) The invariant mass of the proton and the $\pi^{+}$across the whole kinematic range of the whole dataset. The shaded area shows the area that is associated with $\Delta^{++}$. Its peak clearly sits upon large background which includes $\rho^{0}$ events.

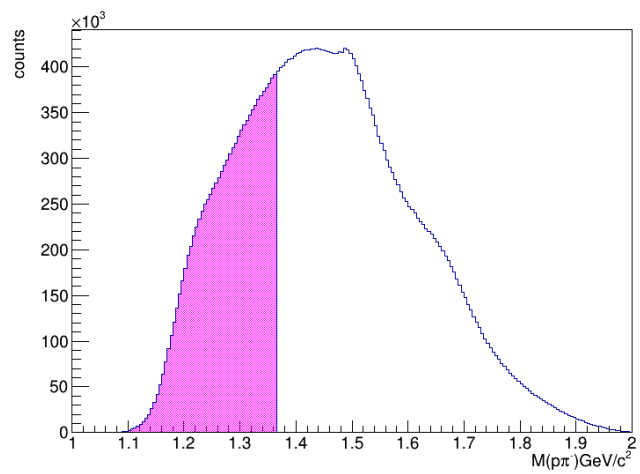

(b) The invariant mass of the proton and the $\pi^{-}$across the whole kinematic range of the whole dataset. The shaded area shows the area that is associated with $\Delta^{0}$. Its peak is not even clear due to the large amount of background which includes $\rho^{0}$ events.

Figure 6.27: Invariant mass of the other two contribution channels for the whole dataset.

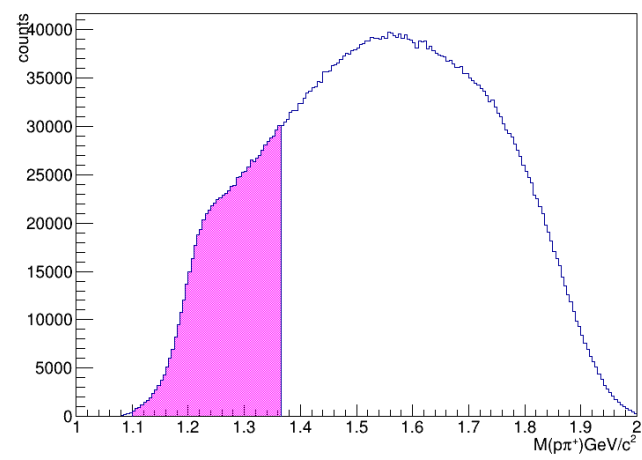

(a) The invariant mass of the proton and the $\pi^{+}$ across the whole kinematic range of the 2100 $\mathrm{MeV}$ dataset. The shaded area shows the area that is associated with $\Delta^{++}$.

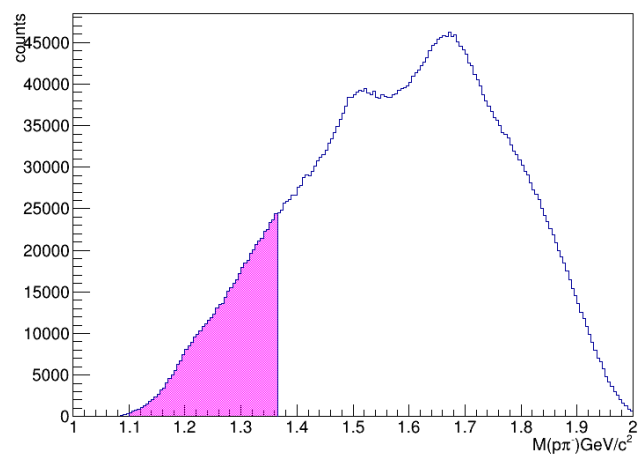

(b) The invariant mass of the proton and the $\pi^{-}$across the whole kinematic range of the $2100 \mathrm{MeV}$ dataset. The shaded area shows the area that is associated with $\Delta^{0}$. Seen here are the other hadronic resonances, $\mathrm{N}(1520)$ and $\mathrm{N}(1685)$.

Figure 6.28: Invariant mass of the other two contribution channels for the $2100 \mathrm{MeV}$ dataset only. 


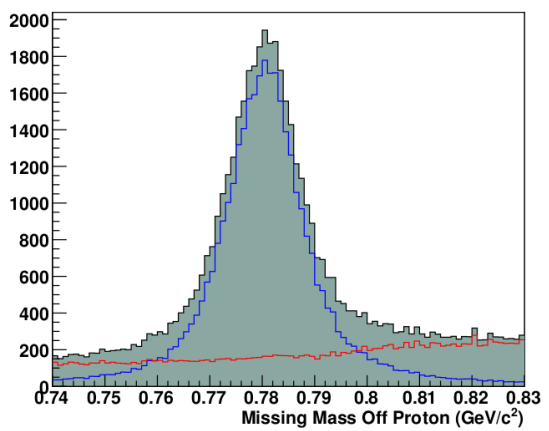

(a) The signal background separation for $\omega$ mesons using the g1c dataset from CLAS.

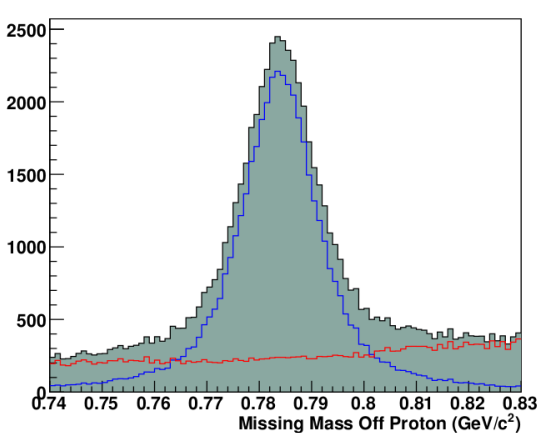

(b) The signal background separation for $\omega$ mesons using the g8b dataset from CLAS, the same dataset used in this analysis.

Figure 6.29: An example of the standard fitting technique used to separate the mesonic signal and the background for photoproduced $\omega$ mesons used in an analysis to measure the cross section and SDMEs of photoproduced $\omega$ mesons at CLAS [119]. Here the Q-factor fitting technique using likelihood functions was used instead of simple binned fitting but this technique still requires a fairly simple background and signal separation and a well defined likelihood function to describe the two contributions.

$\omega$ mesons. In the next stage, the polarization observable under study is analysed for the areas left and right of the signal area. If the values for both background areas are comparable, it is presumed that the background contribution in the signal area is the same. Then a dilution factor can be calculated by taking the integrals of the background and the signal contributions to the fit; equation 6.3 shows the relationship between a measured beam asymmetry and the contributions from the signal and background.

$$
\Sigma_{M} N_{M}=\Sigma_{S} N_{S}+\Sigma_{B C G} N_{B C G}
$$

Where:

- $\Sigma_{M}$ is the extracted asymmetry from the procedure described in the following section.

- $N_{M}$ is the total number of events in the distribution used to extract $\Sigma_{M}$.

- $\Sigma_{S}$ is the actual value of interest.

- $N_{S}$ is the number of events associated with the signal which is taken as the 
integral of the signals contribution to the fit.

- $\Sigma_{B C G}$ is the contribution to the measured asymmetry from the background, which is considered everything except the signal under investigation.

- $N_{B C G}$ is the number of events associated with the background which is taken as the integral of the background contributions to the fit.

Ideally the contribution from the background is zero in the region of interest and then:

$$
\Sigma_{S}=\Sigma_{M} \frac{N_{M}}{N_{S}}
$$

For this analysis, however, it is not so simple. Calculating a dilution factor is not possible for two reasons. The first is that the underlying resonant contributions have non-zero and varying contributions (this is shown in 6.30) and they cannot be distentagled with kinematic cuts. The second is that the standard beam asymmetry extraction technique cannot be used here, this is discussed below in section 6.4 , and the technique used requires the data to be separated with regards to more kinematic variables than usual. This results in a statistics problem when trying to find regions which can reasonably be called background dominant.

As a result of this, the dilution factor technique is used to quantify the uncertainty associated with the extracted beam asymmetry values rather than alterting the actual extracted values. The $\rho^{0}$ signal is a superimposition of many resonant channels all with an unknown contribution to the beam asymmetry. These cannot be easily quantified. What is needed are cross sections for each channel associated with two pion photoproduction and a means of separating the channels, e.g. a partial waves analysis. In the absence of these two things, the situations where the background contribution, $\Sigma_{B C G}$, is either completely positive or completely negative $\left(\Sigma_{B C G} \pm 1\right)$ are used to quantify the upper and lower limits of the extracted beam asymmetry values for the $\rho^{0}$ and the $f^{0}$ : 


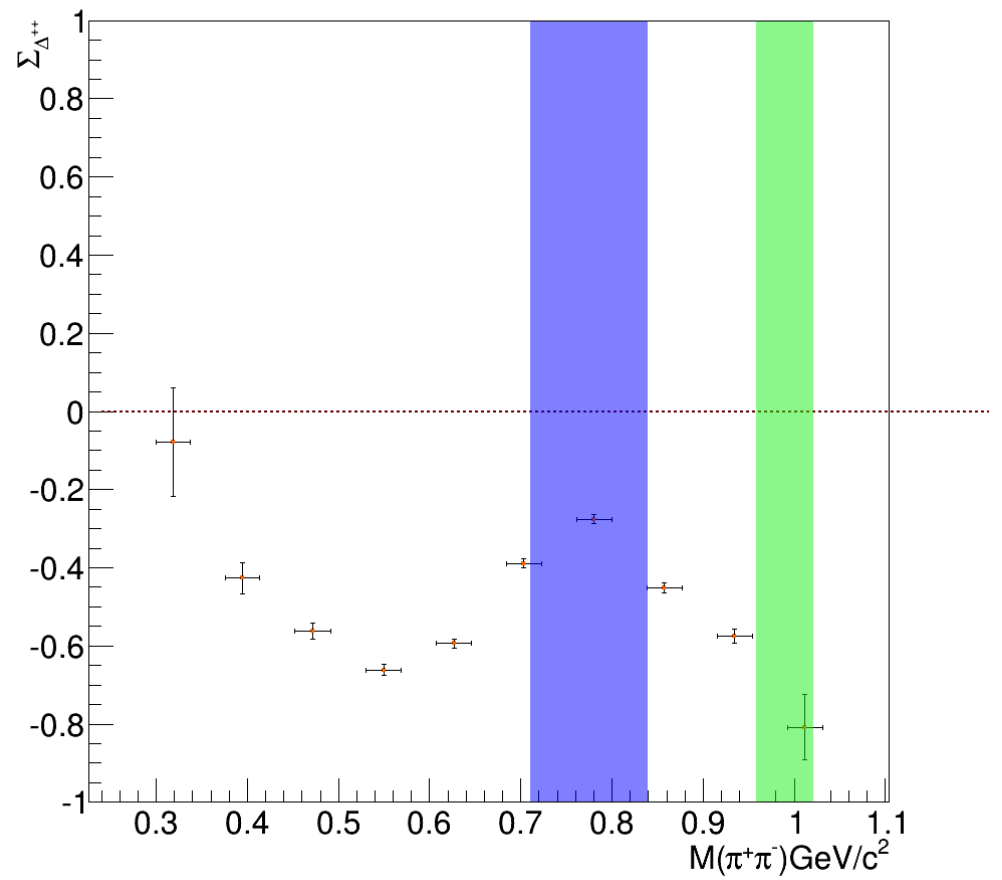

Figure 6.30: Example of the asymmetry for the $\Delta^{++}$as a function of the invariant mass of the two pions the $\mathrm{W}$ region $\left(1.90 \leq \mathrm{W}<1.95\left(\mathrm{GeV} / \mathrm{c}^{2}\right)\right)$ and the $\cos \theta_{C M}$ region $\left(0.601 \geq \cos \theta_{C M}>0.461\right)$ The region highlighted in blue is associated with the $\rho^{0}$ meson and green with the $f^{0}$ meson. Most important is the strong non-zero contribution preceding the $\rho^{0}$ meson signal. 


$$
\Sigma_{\text {err. }}=\left|\Sigma_{M}-\frac{\Sigma_{M} N_{M} \pm N_{B C G}}{N_{S}}\right|
$$

\subsection{1 $\Delta$ Cuts and Kinematic Binning Selection}

The Dalitz plots in figure 6.32 and in figure 6.33 show the signal of the $\Delta \mathrm{s}$ in the lower centre of mass energy (W) regions, especially in figure 6.32. The effects of cutting on the regions associated with the two $\Delta$ resonances, highlighted in magenta in figure 6.27 and the cut shown on Daltiz plots for the entire dataset in figure 6.31, are shown in figure 6.34. The main effect is the removal of events in the region of the $\rho^{0}$ and in the backward region, where it is expected resonance sensitivity will be at its highest. Seeming as it is resonances that we are interested in, it wasn't considered gainful to cut these events despite simplifying the analysis by doing so.

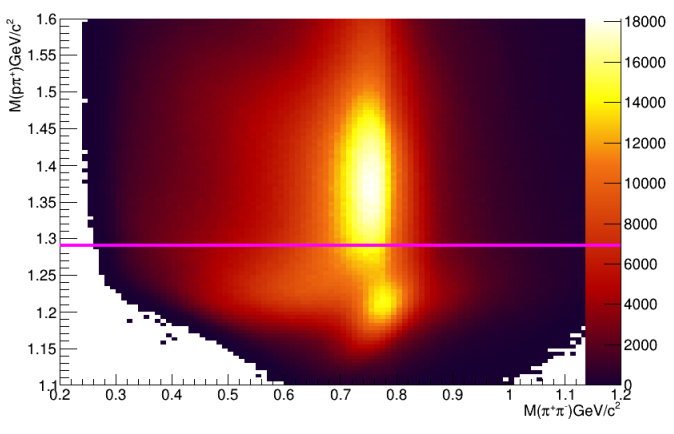

(a) The Dalitz plot of the reconstructed invariant mass of the proton and $\pi^{+}$against the mass of the two pions for the whole dataset. The line shown highlights where the cut would be applied to remove events associated with the $\Delta^{++}$resonance. A faint line centered at $\sim 1.23$ $\mathrm{GeV}$ on the y-axis shows the contribution from the $\Delta^{++}$. The signal at $1.2 \mathrm{GeV}$ on the y-axis and $0.78 \mathrm{GeV}$ on the $\mathrm{x}$-axis is the contribution from the $\omega(782)$ which decays to $\pi^{+} \pi^{-} \pi^{0}$. Some undetected neutral pions get through our cuts due to the semi-inclusive channels.

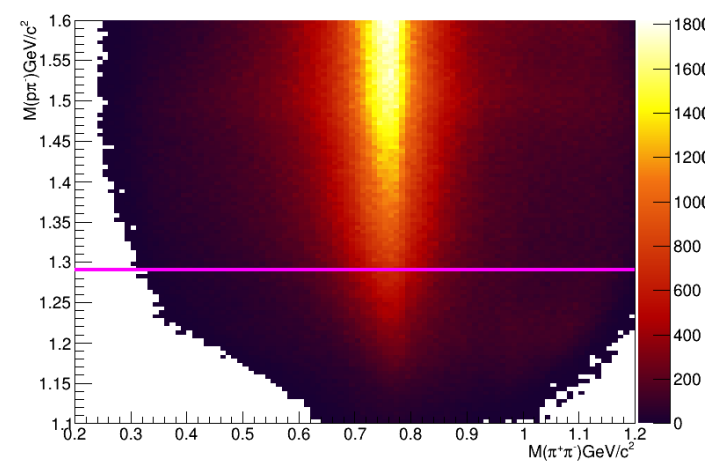

(b) The Dalitz plot of the reconstructed invariant mass of the proton and $\pi^{-}$against the mass of the two pions. The line shown highlights where the cut would be applied to remove events associated with the $\Delta^{0}$ resonance, which does not have an appreciable signal here.

Figure 6.31: The coarse cut on the $\Delta$ resonances. 


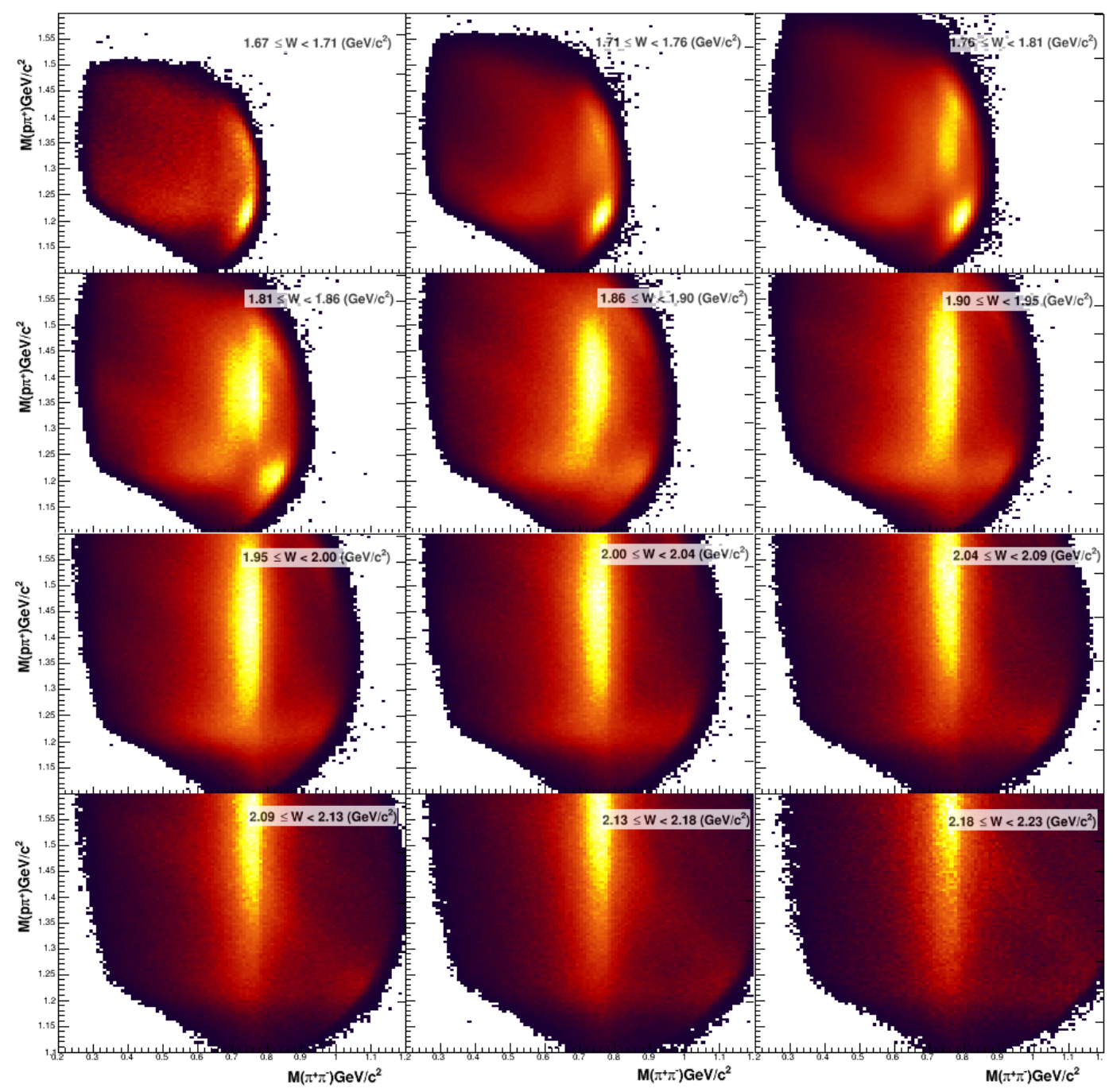

Figure 6.32: The Dalitz plots of the invariant mass of proton and the $\pi^{+}$against that of the two pions for different $W$ regions. The lower $W$ region plots have a clearer contribution from the $\Delta^{++}$. The fifth plot shows the start of the kinematic region we have used for the remaining analysis. In these regions the $\rho^{0}$ is dominant. The $f^{0}$ signal isn't appreciable until the data is separated into different $\cos \theta_{C M}$ bins. 


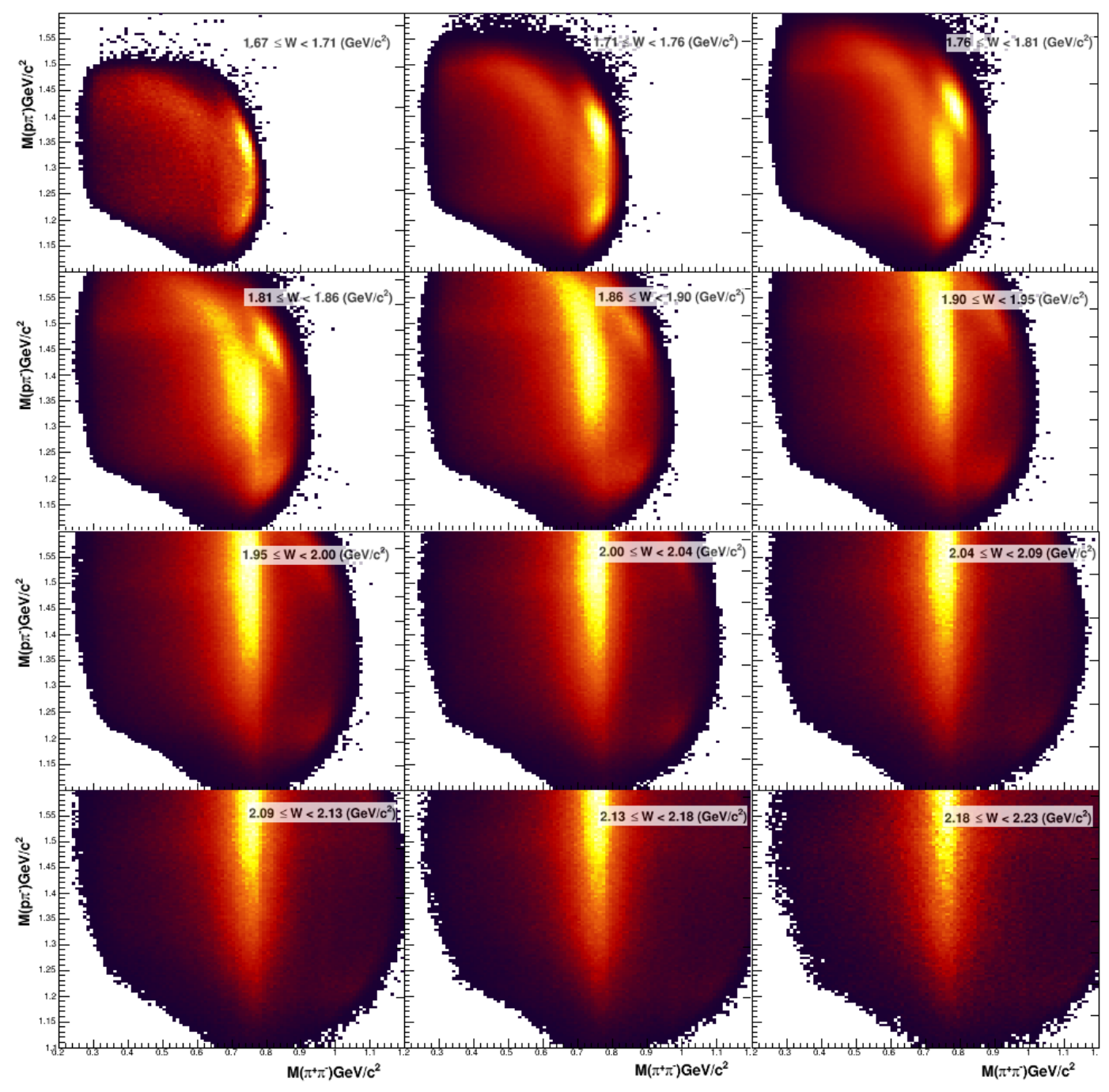

Figure 6.33: The Dalitz plots of the invariant mass of proton and the $\pi^{-}$against that of the two pions for different $W$ regions. The lower $W$ region plots show the lack of a clean $\rho^{0}$ signal; this is due to the fact that the threshold energy for $\rho^{0}$ production is $\mathrm{W} \sim 1.7 \mathrm{GeV}$. 


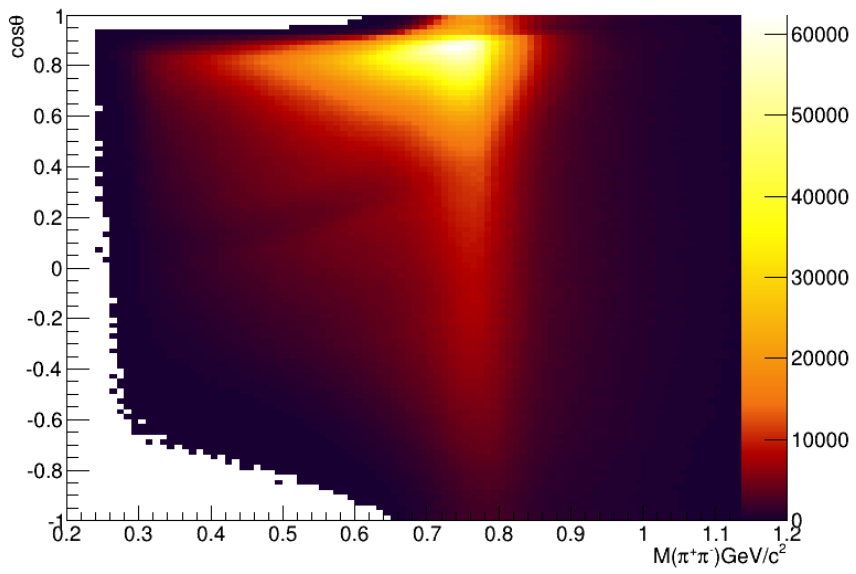

(a) The invariant mass of the two pions with respect to $\cos \theta_{C M}$ without any cuts on the $\Delta \mathrm{s}$.

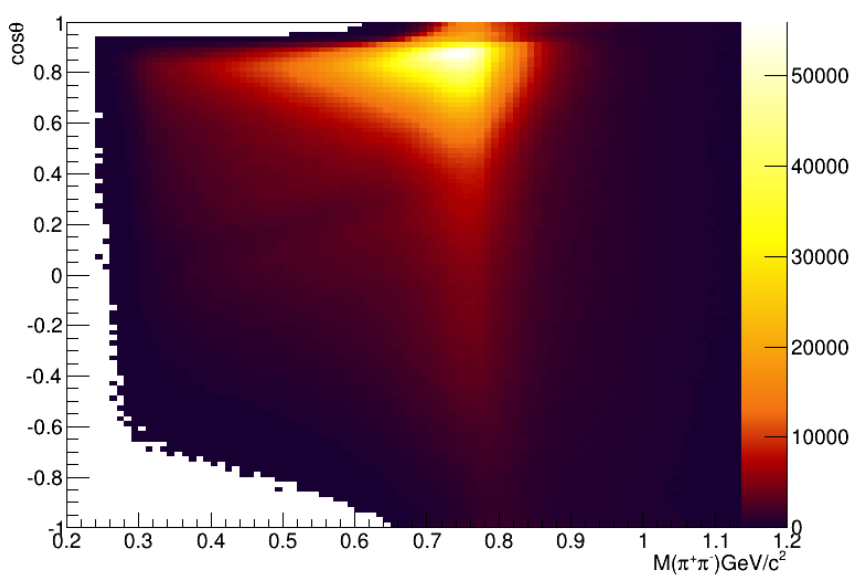

(b) The invariant mass of the two pions with respect to $\cos \theta_{C M}$ with just the cut on the $\Delta^{++}$. A clear loss of events in the backward region can be seen.

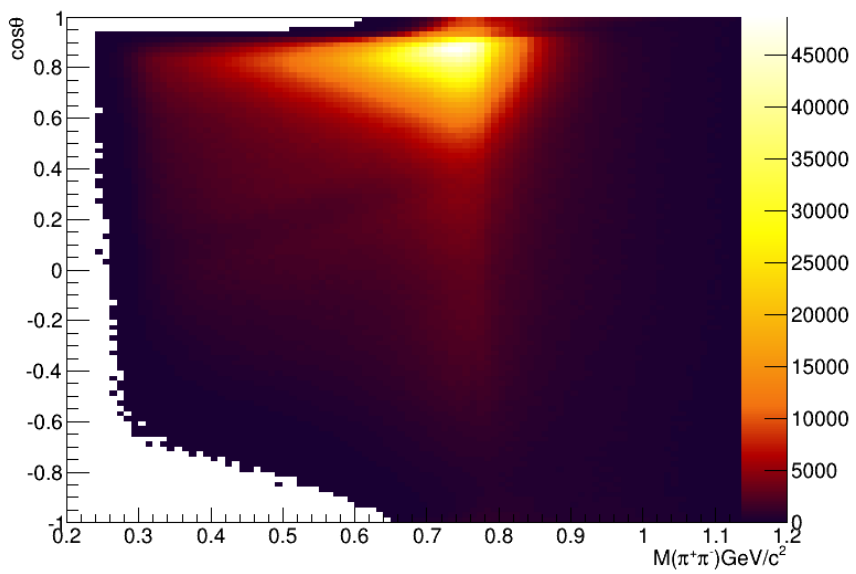

(c) The invariant mass of the two pions with respect to $\cos \theta_{C M}$ with both the cut on the $\Delta^{++}$and the $\Delta^{0}$. A further loss of events in the backward region can be seen.

Figure 6.34: The effect of cutting the regions associated with the $\Delta$ resonances. 105 
In order to deal with the $\Delta \mathrm{s}$ to a certain degree, the remaining analysis was constricted to $\mathrm{W}$ regions above $1.85 \mathrm{GeV}$. Figures 6.32 and 6.33 show that above this energy the $\rho^{0}$ signal is much cleaner. In fact the threshold energy for $\rho^{0}$ photoproduction is $1.7 \mathrm{GeV}$ so the lowest energy region would have been cut anyway. Also, the W regions below 1.80 have incident photons with energies below the nominal setting for the lowest dataset $(1300 \mathrm{MeV})$. This includes the first two $\mathrm{W}$ regions shown in figure 6.32 and 6.33. This is only a problem as the polarization values for the photon beam diminish the further the energy of the incident photon is from the coherent edge setting.

At this point the kinematic binning was decided by integrating the whole dataset in terms of $\cos \theta_{C M}$ and dividing it into ten separate bins with approximately the same number of events. This is shown in figure 6.35. Then the same was done for the dataset with respect to the centre of mass energy. It is obvious that certain $\mathrm{W}$ regions will have a different $\cos \theta_{C M}$ distribution and without taking that into consideration when choosing the regions will result in an inbalance between the number of events in certain kinematic bins. However, for the sake of clarity when studying the final results it was considered more desirable to mainatin a consistent binning across $\cos \theta_{C M}$ for each $\mathrm{W}$ region as the extracted asymmetries were ultimately to be shown with respect to $\cos \theta_{C M}$. Figure 6.36 shows the integration process for selecting the $\mathrm{W}$ binning regions. The resulting kinematic regions are shown in table 6.3 and table 6.4 , for $\cos \theta_{C M}$ and $\mathrm{W}$ respectively. 


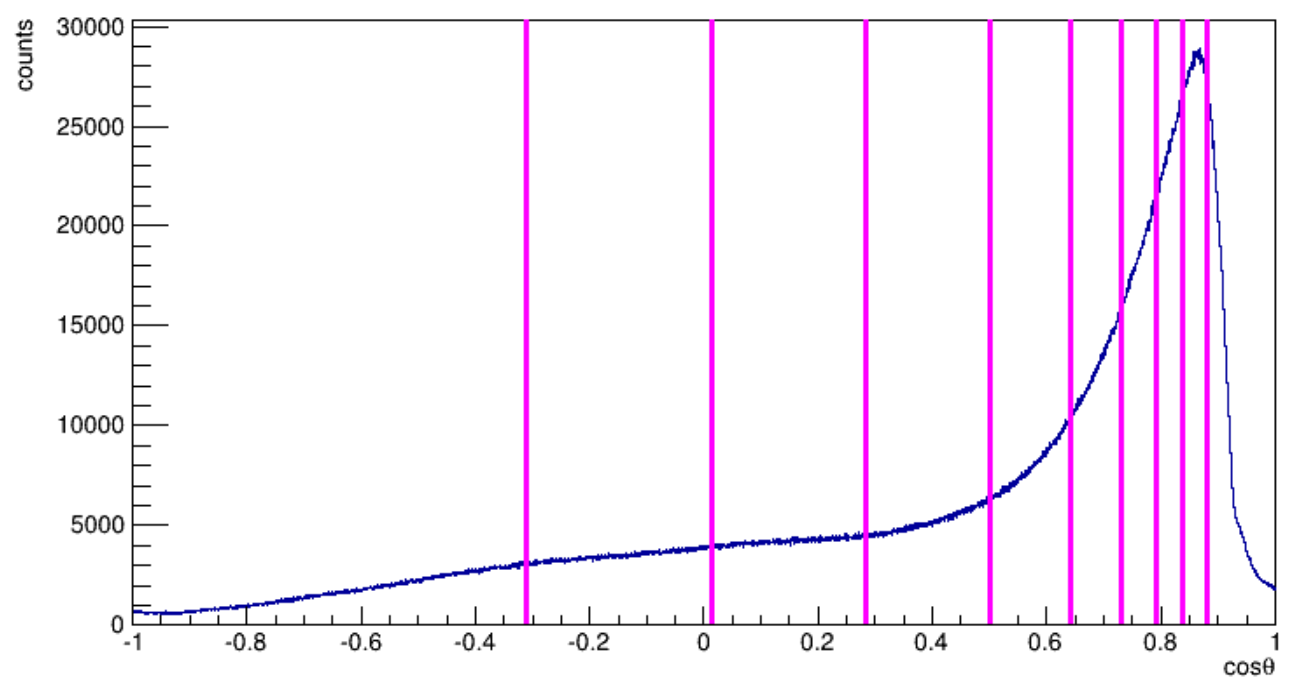

Figure 6.35: The $\cos \theta_{C M}$ distribution for the dataset spanning centre of mass region $1.85-2.23 \mathrm{GeV}$. Each bin contains approximately one tenth of the entire number of events and are separated by magenta lines.

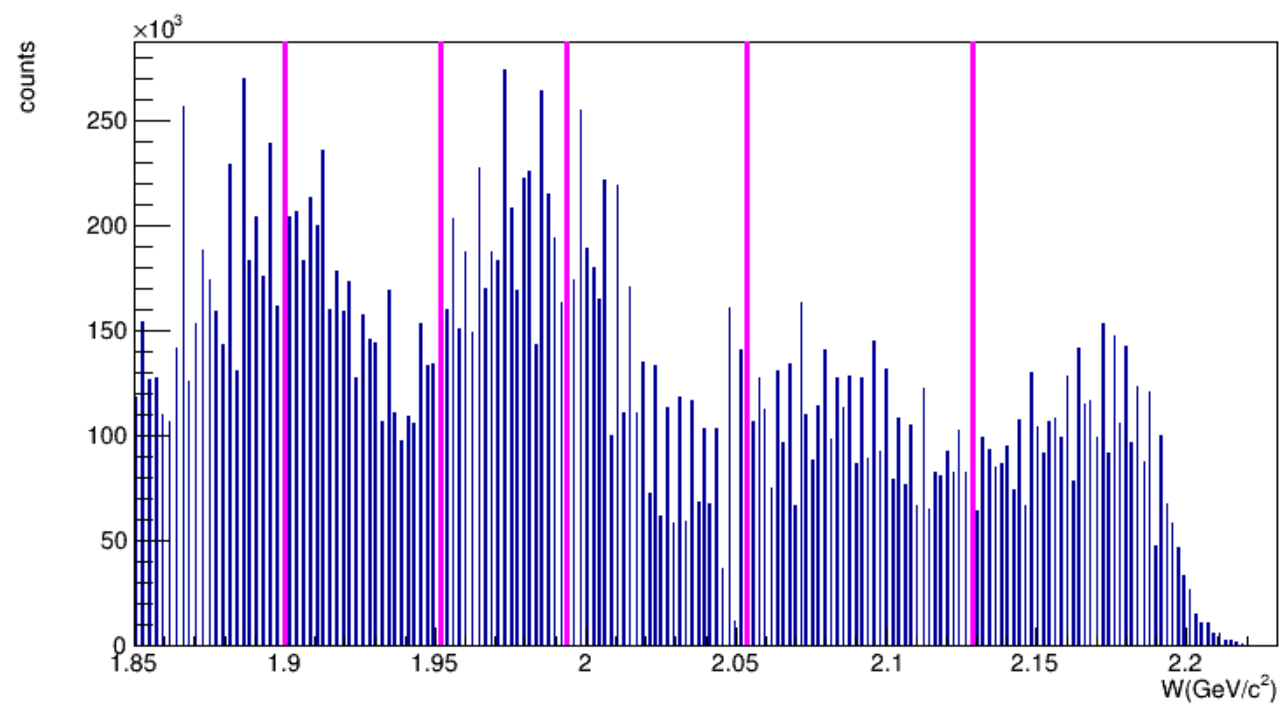

Figure 6.36: The $\mathrm{W}$ distribution for the dataset spanning the entire $\cos \theta_{C M}$ distribution. Each bin contains approximately one sixth of the entire number of events and are separated by magenta lines. 


\begin{tabular}{|l|c|c|}
\hline $\cos \theta_{C M}$ & Lower Limit $\left(\mathrm{GeV} / c^{2}\right)$ & Upper Limit $\left(\mathrm{GeV} / c^{2}\right)$ \\
\hline Region 1 & $>0.874$ & $\leq 1.000$ \\
\hline Region 2 & $>0.823$ & $\leq 0.874$ \\
\hline Region 3 & $>0.768$ & $\leq 0.823$ \\
\hline Region 4 & $>0.698$ & $\leq 0.768$ \\
\hline Region 5 & $>0.601$ & $\leq 0.698$ \\
\hline Region 6 & $>0.461$ & $\leq 0.601$ \\
\hline Region 7 & $>0.263$ & $\leq 0.461$ \\
\hline Region 8 & $>0.014$ & $\leq 0.263$ \\
\hline Region 9 & $>-0.303$ & $\leq 0.014$ \\
\hline Region 10 & $\geq-1.000$ & $\leq-0.303$ \\
\hline
\end{tabular}

Table 6.3: $\cos \theta_{C M}$ kinematic bins used in the analysis.

\begin{tabular}{|l|c|c|}
\hline W & Lower Limit $\left(\mathrm{GeV} / c^{2}\right)$ & Upper Limit $\left(\mathrm{GeV} / \mathrm{c}^{2}\right)$ \\
\hline Region 1 & $\geq 1.85$ & $<1.90$ \\
\hline Region 2 & $\geq 1.90$ & $<1.95$ \\
\hline Region 3 & $\geq 1.95$ & $<2.00$ \\
\hline Region 4 & $\geq 2.00$ & $<2.05$ \\
\hline Region 5 & $\geq 2.05$ & $<2.13$ \\
\hline Region 6 & $\geq 2.13$ & $\leq 2.23$ \\
\hline
\end{tabular}

Table 6.4: W kinematic bins used in the analysis.

\subsection{Fitting $\mathbf{M}\left(\pi^{+} \pi^{-}\right)$Distributions}

The fitting technique used to describe the mass distrubutions of the two pions incorporates a Monte Carlo simulation in order to describe the shape of these background processes projected onto the two pion invariant mass distributions. The fit- 
ting process is similar to that developed in an electroproduction experiment using CLAS [120][121]. The shape of the background in the two pion invariant mass plots is a combination of the contribution from the $\Delta \mathrm{s}$ along with that of the non-resonant contribution. This is combined with the skewed breit-wigner fits for the $\rho^{0}$ and the $f^{0}(980)$. The fit used takes the form of:

$$
\frac{d N}{d M_{\pi^{+} \pi^{-}}}=s B W_{\rho^{0}}\left(M_{\pi^{+} \pi^{-}}\right)+s B W_{\rho^{0}}\left(M_{\pi^{+} \pi^{-}}\right)+M_{b c g}\left(M_{\pi^{+} \pi^{-}}\right)
$$

Where:

$$
M_{b c g}\left(M_{\pi^{+} \pi^{-}}\right)=M_{\Delta^{++} \pi^{-}}\left(M_{\pi^{+} \pi^{-}}\right)+M_{\Delta^{0} \pi^{+}}\left(M_{\pi^{+} \pi^{-}}\right)+M_{p \pi^{+} \pi^{-}}\left(M_{\pi^{+} \pi^{-}}\right)
$$

Standard p-wave Breit-Wigner shapes are used with a skewing factor. This is to account for the interference between non-resonant contributions and the $\rho^{0}$ mechanism [122][123]. It has the following form:

$$
s B W_{\rho^{0}}\left(M_{\pi^{+} \pi^{-}}\right)=B W_{\rho^{0}}\left(M_{\pi^{+} \pi^{-}}\right)\left(\frac{M_{\rho^{0}}}{M_{\pi^{+} \pi^{-}}}\right)^{n_{\text {skew }}}
$$

There are 9 parameters: the weight for the Breit-Wigner shapes of the $\rho^{0}$ and of the $f^{0}$; the mean mass of each meson; the width of each meson; the skewing factor for each of the meson's Breit-Wigner; and the weight of the background contributions from the $\Delta s$ ' projection on to the two pion invariant mass plots and the phase space contributions. It was found that leaving the parameters completely free resulted in unsuccessful fits. The mass and width parameters were set with limits according to the values stated in the PDG [51]. The skewing factors were set to be within 0 and 10 as the value for the $\rho^{0}$ is predicted to be $\sim 4$ by Ross and Stodolsky [122]. This means that only the weight factors were without limits. Examples of this fit applied to the extremity of the data's kinematical region are show in figure 6.37 and in appendix A for the entire set of kinematic bins. 


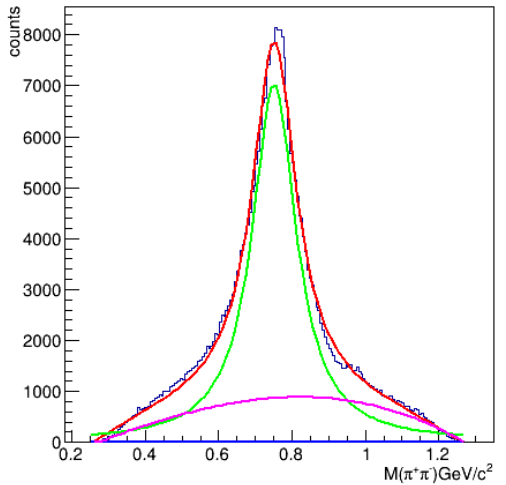

(a) The invariant mass of the two pions in the forward angle region and low $\mathrm{W}$ with the fit applied as described above. Here the $\rho^{0}$ signal is most prominent.

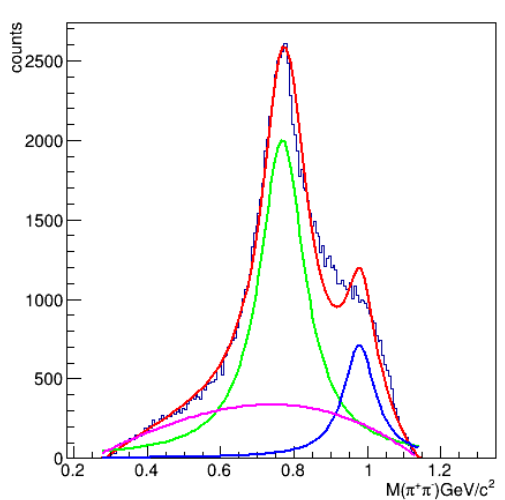

(b) The invariant mass of the two pions in the backward angle region and high $\mathrm{W}$ with the fit applied as described above. Here the $f^{0}$ signal can been seen in addition to the $\rho^{0}$ signal.

Figure 6.37: Invariant mass of the two pions in different kinematic regions fitted with the function shown in equation 6.6

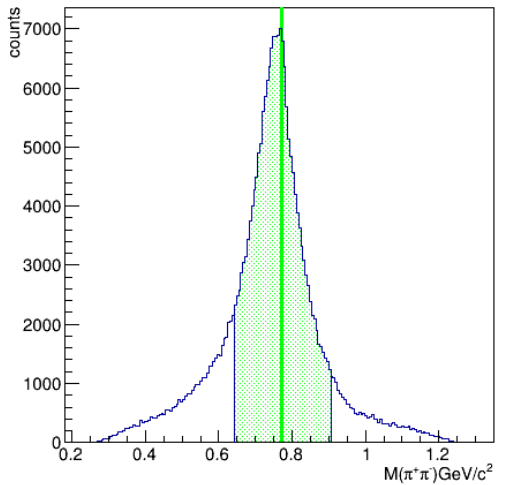

(a) The region considered to be sufficiently $\rho^{0}$ dominant for the forward angle and low $W$ region is highlighted in green. No contribution from the $f^{0}$ is seen is this kinematic region.

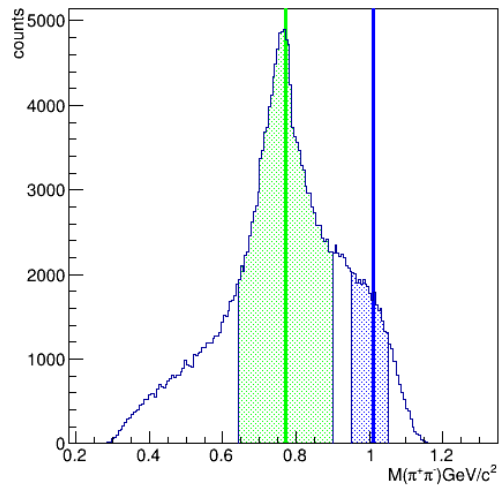

(b) The region considered to be sufficiently $\rho^{0}$ dominant for the backward angle and high $W$ region is highlighted in green and the region considered to be sufficiently $f^{0}$ is highlighted in blue.

Figure 6.38: The region for different kinematic bins used for extracting the beam asymmetry in the region of the $\rho^{0}$ and $f^{0}$. 


\subsection{Beam Asymmetry Extraction}

The region of the two pion invariant which we used for analysing the beam asymmetry of the $\rho^{0}$ and the $f^{0}$ are shown in figure 6.38. The uncertainty associated with the dilution of the background, as discussed above in section 6.2 , is combined with the statistical error of each kinematic bin, and ultimately it is combined with the uncertainty associated with the fitting procedure used to extract the beam asymmetry from the angular distributions. The following notion for the angles involved are:

- $\Phi$ is the azimuthal angle of the $\rho^{0}$ meson in the centre-of-mass frame.

- $\phi_{h}$ is the azimuthal angle of the $\pi^{+}$in the helicity frame.

- $\cos \theta_{h}$ is the cosine of the polar angle of the $\pi^{+}$in the helicity frame.

This section will discuss the procedure used to extract the beam asymmetries of the $\rho^{0}$ meson, the $f^{0}$ meson, and the decay pions. 


\subsubsection{Basic 1-D Technique}

\section{$(\vec{\gamma} \mathrm{p})$ c.m. frame}
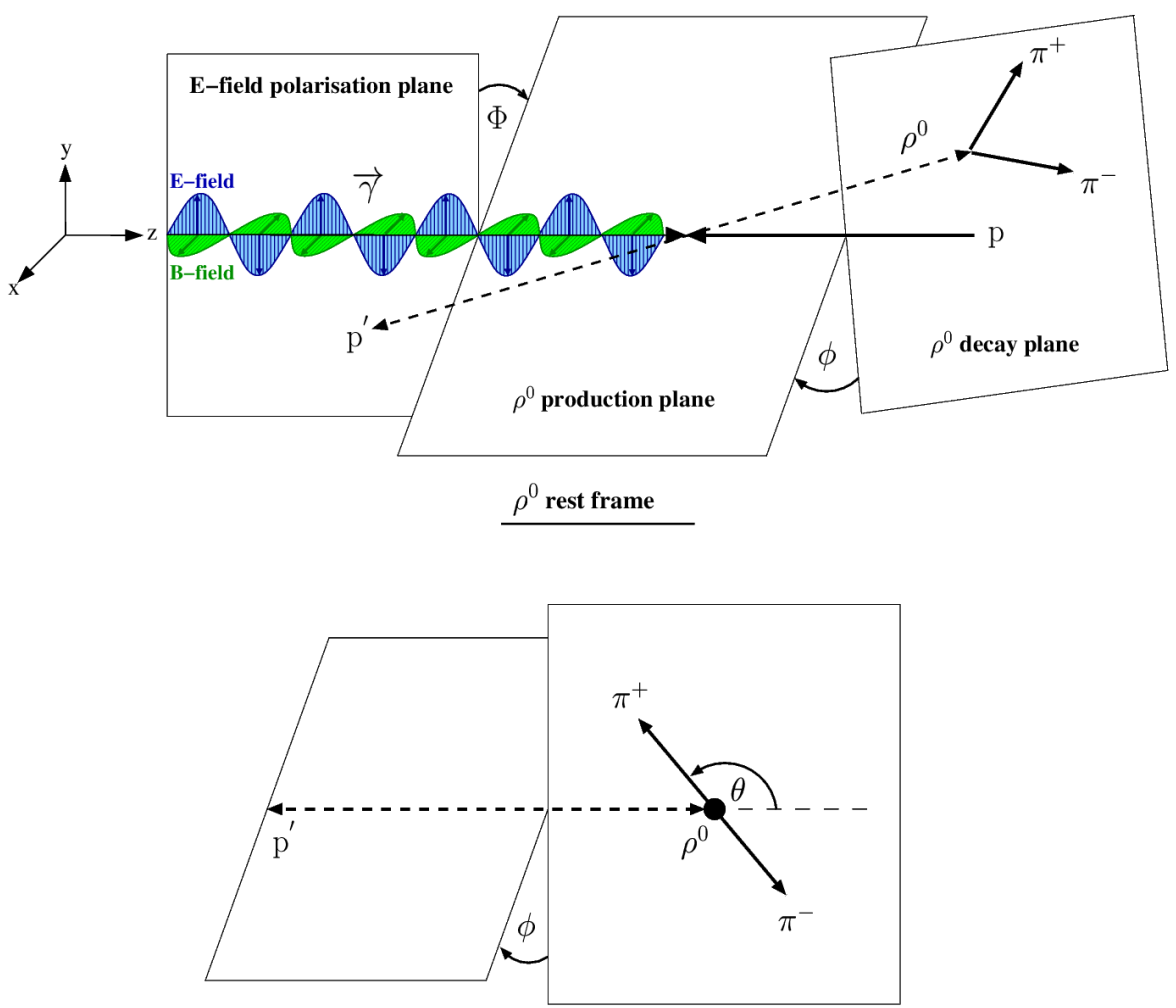

Figure 6.39: A diagram showing the different planes and angles involved in the photoproduction of $\rho^{0}$ mesons is shown again in this section for ease of reference [59].

The standard technique used to extract the beam asymmetry for mesons photoproduced using a linearly polarized beam is an extension of the function derived in section 2.5 where the angular distribution is integrated over the angles of the positive pion in the helicity frame. Figure 6.39 is shown again in this section for ease of reference when discussing the different angles involved. Equation 2.32 derived in section 2.5 has the form:

$$
N=1 \pm P_{\gamma} \Sigma \cos (2 \Phi)
$$

Where $\Phi$ is the azimuthal angle of the $\rho^{0}$ meson in the production plane, $P_{\gamma}$ is the degree of polarization of the photon beam determined using the procedure described 
above in section 5.5, and $\mathrm{N}$ is the number of events as a function of $\Phi$. This form is for the case where only polarized photons are present in the experiment. In reality there is still an unpolarized component to the angular distribution. In addition to this, an acceptance correction must be taken into account, so the true form describing the angular distribution is:

$$
N=\operatorname{Acc}(\Phi) N_{0}\left(1 \pm P_{\gamma} \Sigma \cos (2 \Phi)\right)
$$

Where $N_{0}$ is the unpolarized distribution and $\operatorname{Acc}(\Phi)$ is the detector acceptance of the $\Phi$ distribution. We can take an asymmetry of the two different plane settings used in this experiment, parallel and perpendicular to the xz-plane of the laboratory frame:

$$
N_{A}=\frac{N_{\|}-N_{\perp}}{N_{\|}+N_{\perp}}
$$

By looking at an asymmetry of these two settings, the acceptance and unpolarized contributions cancel out. This results in the following function to describe these asymmetry angular distributions:

$$
N_{A}=-P_{\gamma} \Sigma \cos (2 \Phi)
$$

However, this does not account for the differences in flux between the two plane settings, nor does it account for the potential difference between the polarization values of these settings, which could potentially be quite different as there were significant intervals between taking data for the different plane settings. Also, the small variations in the diamond which cause a small offset from the ideal case of the PARA and PERP settings being exactly orthogonal needs to be included. These considerations result in the following extended form of equation 6.12:

$$
N_{A}=\frac{\left(\frac{N_{\perp}}{N_{\|}}-1\right)-\left(\frac{N_{\perp}}{N_{\|}} P_{\perp}+P_{\|}\right) \Sigma \cos \left(2\left(\Phi-\Phi_{0}\right)\right)}{\left(\frac{N_{\perp}}{N_{\|}}+1\right)-\left(\frac{N_{\perp}}{N_{\|}} P_{\perp}-P_{\|}\right) \Sigma \cos \left(2\left(\Phi-\Phi_{0}\right)\right)}
$$




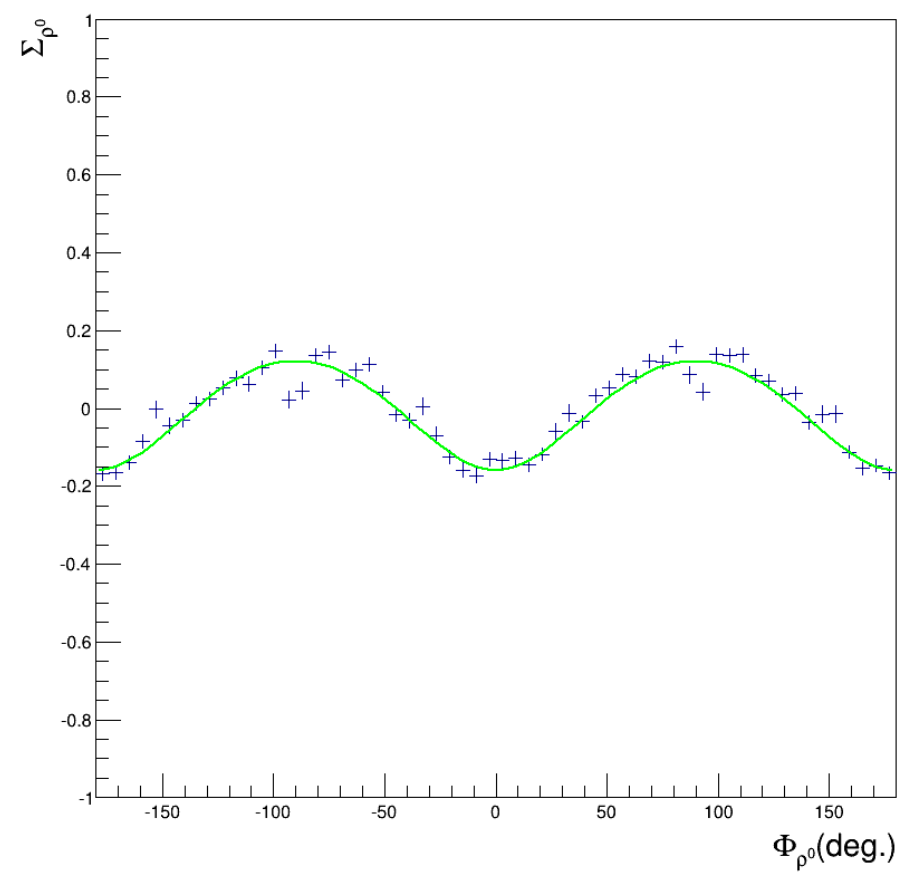

Figure 6.40: An example of the fit described in equation 6.13 applied to an angular distribution asymmetry.

Where $N_{\|}$and $N_{\perp}$ are the flux of the different orientations, $P_{\|}$and $P_{\perp}$ are the mean polarization values of the different orientations, $\Phi_{0}$ is the offset to allow for any misalignment between the diamond and detector reference frames. Although the fit function looks quite complicated, only two variables were actually free parameters; $\Sigma$ and $\Phi_{0}$. An example of this fit applied to an angular asymmetry for the $\rho^{0}$ is shown in figure 6.40 .

\subsection{The Need for a 2-D Fit}

Due to the spin 1 nature of the $\rho^{0}$ meson, the assumption that we can integrate over the pion's helicity angles is not true. The tensor polarization of the $\rho^{0}$ meson affects the anglular distributions of the pions; as we detect the pions and reconstruct our information of the rho from them this effect needs to be accounted for. 
The dependency of the $\rho^{0}$ meson's azimuthal angle on $\cos \theta_{h}$ and $\phi_{h}$ were investigated in order to see if we could integrate over either of them. Figure 6.41 shows $\Phi$ as a function of $\cos \theta_{h}$ in two different azimuthal regions of the pion in the helicity frame. Figure 6.41a is integrated over a small $\phi_{h}$ region centred around zero and a consist $2 \Phi$ distribution can be seen suggesting that we can integrate over $\cos \theta_{h}$. Figure $6.41 \mathrm{~b}$ is centred around 144 degrees and shows a consist offset further suggesting that there is no $\cos \theta_{h}$ dependency for $\Phi$, but there is one on $\phi_{h}$.

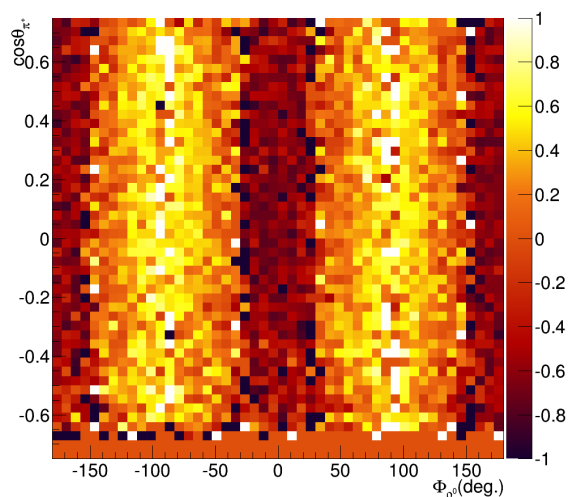

(a) $\Phi$ as a function of $\cos \theta_{h}$ for the region of $\phi_{h}$ between -36.0 and 36.0 degrees $\cos \theta_{h}$ is between 0.85 to 1.0. As can be seen here $\Phi$ distribution does not change with respect to $\cos \theta_{h}$.

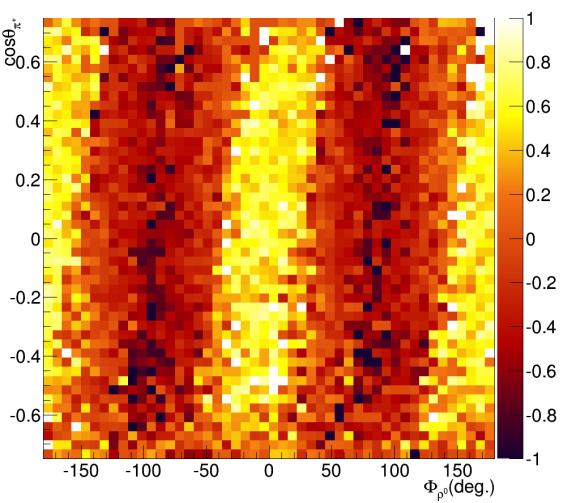

(b) $\Phi$ as a function of $\cos \theta_{h}$ for the region of $\phi_{h}$ between 108 and 180 degrees and $\cos \theta_{h}$ is between 0.85 to 1.0. As can be seen here $\Phi$ distribution changes with respect to $\cos \theta_{h}$ linearly.

Figure 6.41: $\Phi$ as a function of $\cos \theta_{h}$ in different $\phi_{h}$ regions to show the lack of sensitivity of $\Phi$ with respect to $\cos \theta_{h}$.

To further justify the integration over $\cos \theta_{h}$, figure 6.42 shows the PHI as a function of $\phi_{h}$ in two different $\cos \theta_{h}$ regions: the first centered around 0.5 and the second around 0.0. There is no difference between these distributions. 


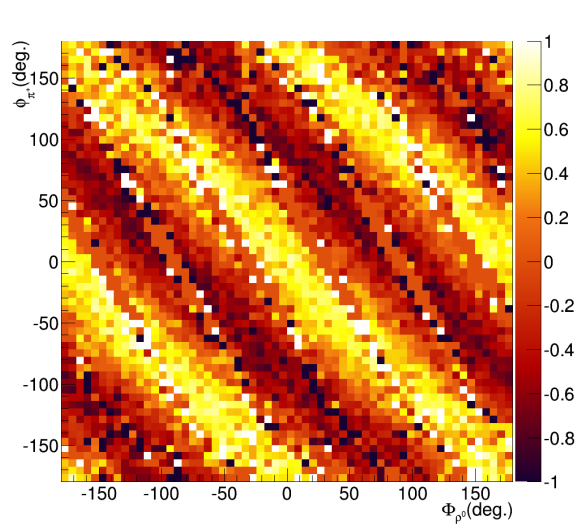

(a) $\Phi$ as a function of $\phi_{h}$ for the region of $\cos \theta_{h}$ between 0.4 and 0.6 and $\cos \theta_{h}$ is between 0.85 to 1.0 . There is clear offset with respect to the $\phi_{h}$.

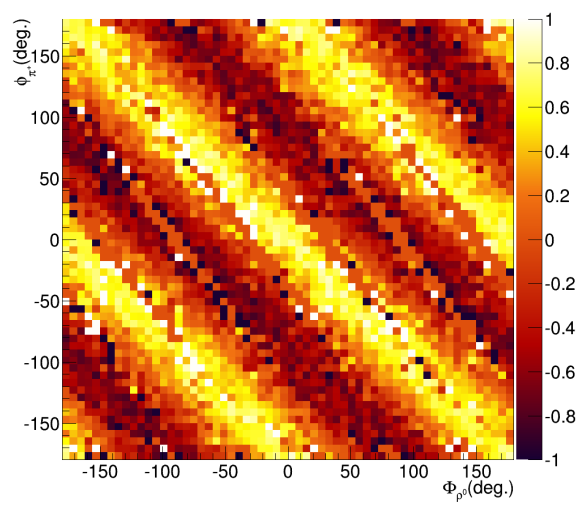

(b) $\Phi$ as a function of $\phi_{h}$ for the region of $\cos \theta_{h}$ between -0.1 and 0.1 and $\cos \theta_{h}$ is between 0.85 to 1.0 . There is the same clear offset with respect to the $\phi_{h}$.

Figure 6.42: $\Phi$ as a function of $\phi_{h}$ in different $\cos \theta_{h}$ to show the lack of sensitivity of $\Phi$ with respect to $\cos \theta_{h}$. The offset relationship between $\Phi$ and $\phi_{h}$ clearly does not change in the different $\cos \theta_{h}$ regions.

As we are interested in extracting the beam asymmetry in this analysis and not other polarization observables, we decided to account for this tensor polarization sensitivity by applying a two dimensional fit to the $\Phi$ versus $\phi_{h}$ asymmetry distributions using the $\phi_{h}$ as an additional offset:

$$
\sigma=\frac{\left(\frac{N_{\perp}}{N_{\|}}-1\right)-\left(\frac{N_{\perp}}{N_{\|}} P_{\perp}+P_{\|}\right) \Sigma \cos \left(2\left(\Phi+\phi_{\pi}-\Phi_{0}\right)\right)}{\left(\frac{N_{\perp}}{N_{\|}}+1\right)-\left(\frac{N_{\perp}}{N_{\|}} P_{\perp}-P_{\|}\right) \Sigma \cos \left(2\left(\Phi+\phi_{\pi}-\Phi_{0}\right)\right)}
$$

This way we can account for the tensor polarization of the $\rho^{0}$ and extract values for the beam asymmetry, without having to develop a much more complex function which would be needed to meaningfully extract any extra information. An example of this fit applied to one of these two dimensional asymmetry distributions is shown in 6.43 . 


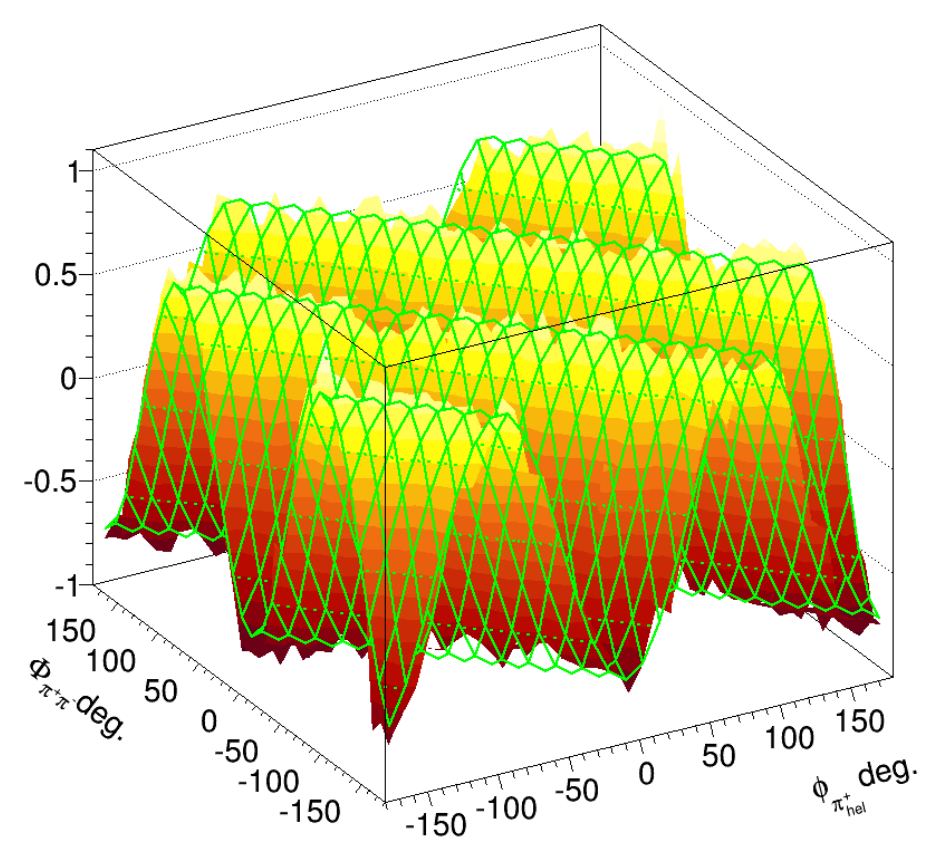

Figure 6.43: An example of the fit described in equation 6.14 applied to a 2-D angular distribution asymmetry of $\Phi$ against $\phi_{h}$.

In addition to the complexity of the inter-angle dependencies, we opted to use semiinclusive channels as well as the exclusive channel as there is an acceptance issue due to the forward angle dominance of the two pion photoproduction (because of the strong diffractive contribution to $\rho^{0}$ production). This is only a problem because there is a hole in the detector in the very forward angle region for removing and inserting targets. This results in a large hole in the $\phi_{h}$ distribution. This can be seen in figure 6.44 where the first plot shows the exclusive channel only and the second shows the exclusive channel along with the semi-inclusive channels where at least two of the final state particles are detected. 


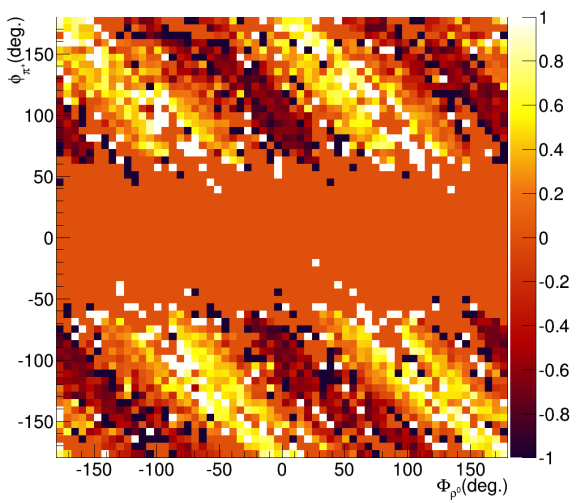

(a) $\Phi$ as a function of $\phi_{h}$ integrated over the full range of $\cos \theta_{h}$ using the exclusive channel. An acceptance issue is seen in the region from -30 to 30 of $\phi_{h}$ and $\cos \theta_{h}$ is between 0.71 to 0.84 .

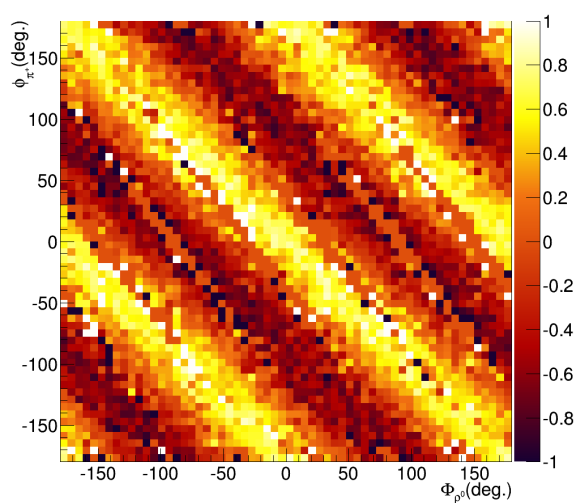

(b) $\Phi$ as a function of $\phi_{h}$ integrated over the full range of $\cos \theta_{h}$ using semi-inclusive channels. The full distribution across $\phi_{h}$ can be seen and $\cos \theta_{h}$ is between 0.71 to 0.84 .

Figure 6.44: $\Phi$ as a function of $\phi_{h}$ integrated over the full range of $\cos \theta_{h}$ using semiinclusive channels and exlcusive channel. The former covers the entirety of the phase space but the latter has a noticable acceptance issue due to the problems with the CLAS detector discussed above.

\subsection{1 $f^{0}$ and Pion Asymmetry Extraction}

As the $f^{0}$ meson is a scalar meson candidate it does not have the same issue as the $\rho^{0}$ meson as it is a spin- 0 particle. Therefore, the one dimensional asymmetry extraction method described above was used for the $f^{0}$ meson. The analysis of the $f^{0}$ meson's beam asymmetry was restricted to certain kinematic regions as no signal is seen in the first $\mathrm{W}$ region nor in the first five $\cos \theta_{C M}$ regions. An example of the fit described above applied to one of the $f^{0}$ asymmetry distributions is shown in 6.45

Similarly, the beam asymmetries for the decay pions were extracting using the one dimensional process. However, these asymmetries will be shown as a function of the invariant mass of the combined pions. It is hoped that these asymmetries will prove sensitive to the signal channels and potentially be used not only to investigate resonance contributions but also to help separate the different wave contributions in a 


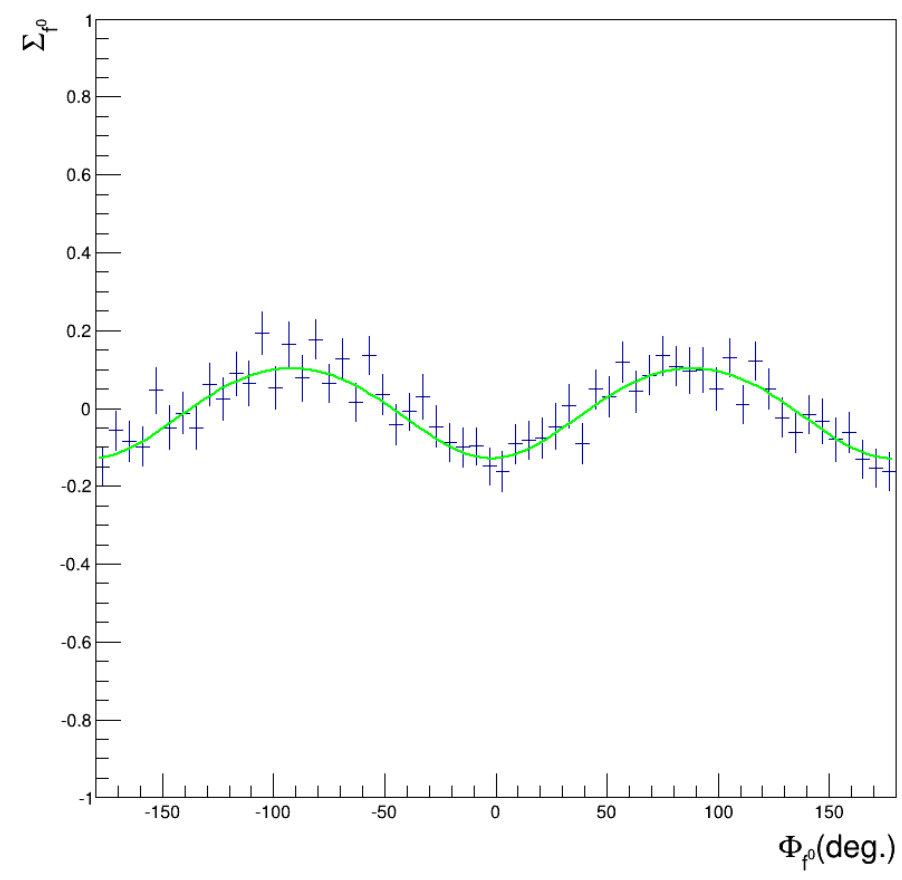

Figure 6.45: An example of the fit described in equation 6.13 applied to an one dimension angular distribution asymmetry of $\phi_{f^{0}}$.

partial wave analysis of this channel. The binning regions of the invariant mass are shown in 6.5 .

\begin{tabular}{|l|c|c|}
\hline$M\left(\pi^{+} \pi^{-}\right)$ & Lower Limit $\left(\mathrm{GeV} / c^{2}\right)$ & Upper Limit $\left(\mathrm{GeV} / c^{2}\right)$ \\
\hline Region 1 & $\geq 0.28$ & $<0.36$ \\
\hline Region 2 & $\geq 0.36$ & $<0.43$ \\
\hline Region 3 & $\geq 0.43$ & $<0.51$ \\
\hline Region 4 & $\geq 0.51$ & $<0.59$ \\
\hline Region 5 & $\geq 0.59$ & $<0.67$ \\
\hline Region 6 & $\geq 0.67$ & $<0.74$ \\
\hline Region 7 & $\geq 0.74$ & $<0.82$ \\
\hline Region 8 & $\geq 0.82$ & $<0.90$ \\
\hline Region 9 & $\geq 0.90$ & $<0.97$ \\
\hline Region 10 & $\geq 0.97$ & $<1.05$ \\
\hline Region 11 & $\geq 1.05$ & $<1.13$ \\
\hline Region 11 & $\geq 1.13$ & $\leq 1.20$ \\
\hline
\end{tabular}

Table 6.5: $\cos \theta_{C M}$ kinematic bins used in the analysis of the pions. 


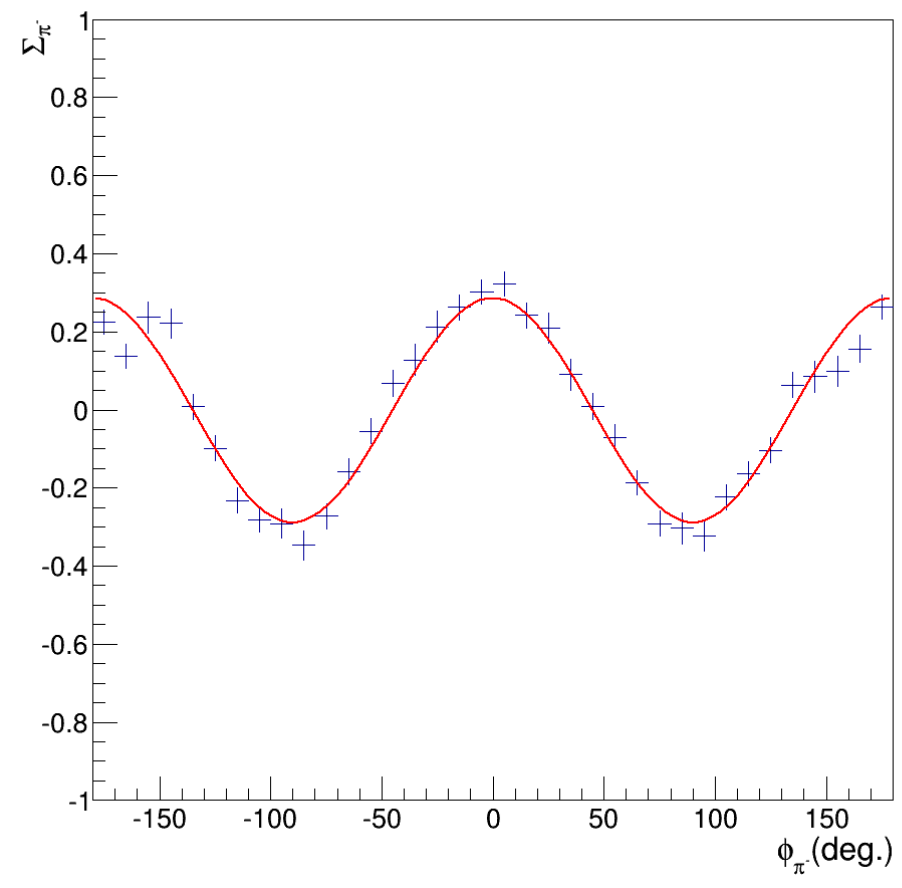

Figure 6.46: An example of the fit described in equation 6.13 applied to an one dimension angular distribution asymmetry of $\phi_{\pi^{+}}$.

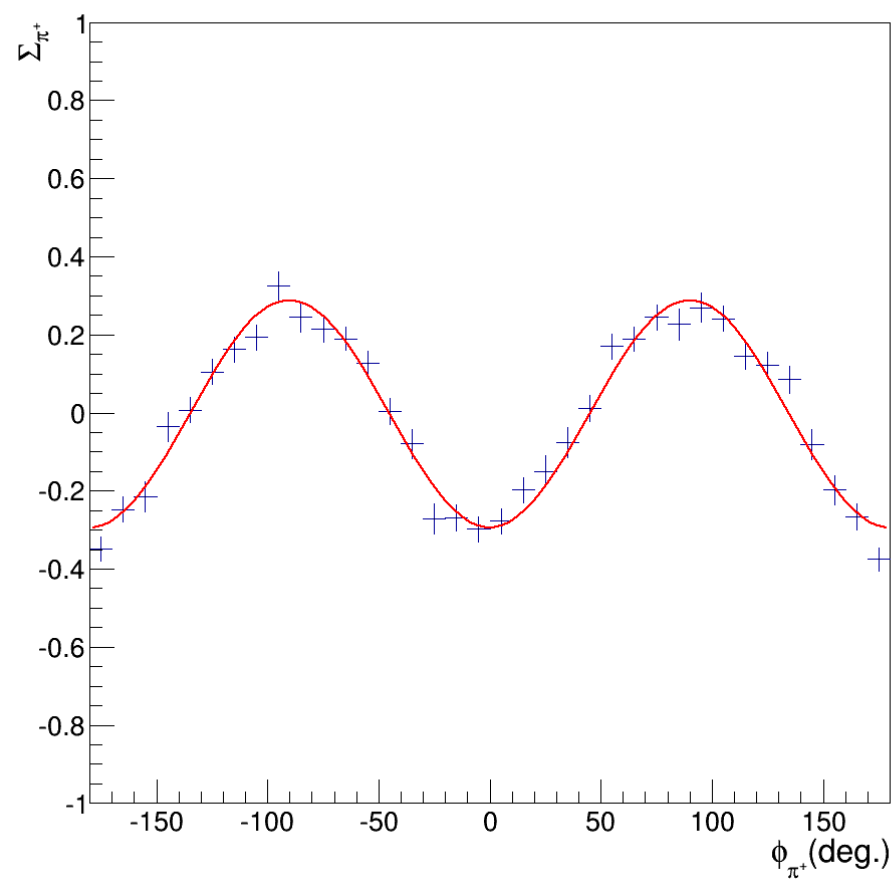

Figure 6.47: An example of the fit described in equation 6.13 applied to an one dimension angular distribution asymmetry of $\phi_{\pi^{+}}$. 


\subsection{Summary}

In this section the separation of $\rho^{0}$ and $f^{0}$ signals from background processes has been discussed, as well as how these separations have been used as part of the uncertainty treatment for our extracted beam asymmetries. Also, the methods used to separate the data into kinematic bins has been shown. And finally, the different methods of extracting beam asymmetries for $\rho^{0}$ mesons, $f^{0}$ meson, and pions have been demonstrated. The following chapter will present the results from these processes. 


\section{Chapter 7}

\section{Results}

The results of the processes discussed in the previous chapter are shown here using the $2-\mathrm{d}$ fit described in section 6.5 to extract the beam asymmetry for the spin- $1 \rho^{0}$ and the 1-d fit described in 6.5.1 for the spin- $0 f^{0}, \pi^{+}$, and $\pi^{-}$. These results will be compared with the most recent cross sections measured for the $\rho^{0}$ and $f^{0}$ in the energy regions closest to those used in this analysis as discussed in chapter 3. 


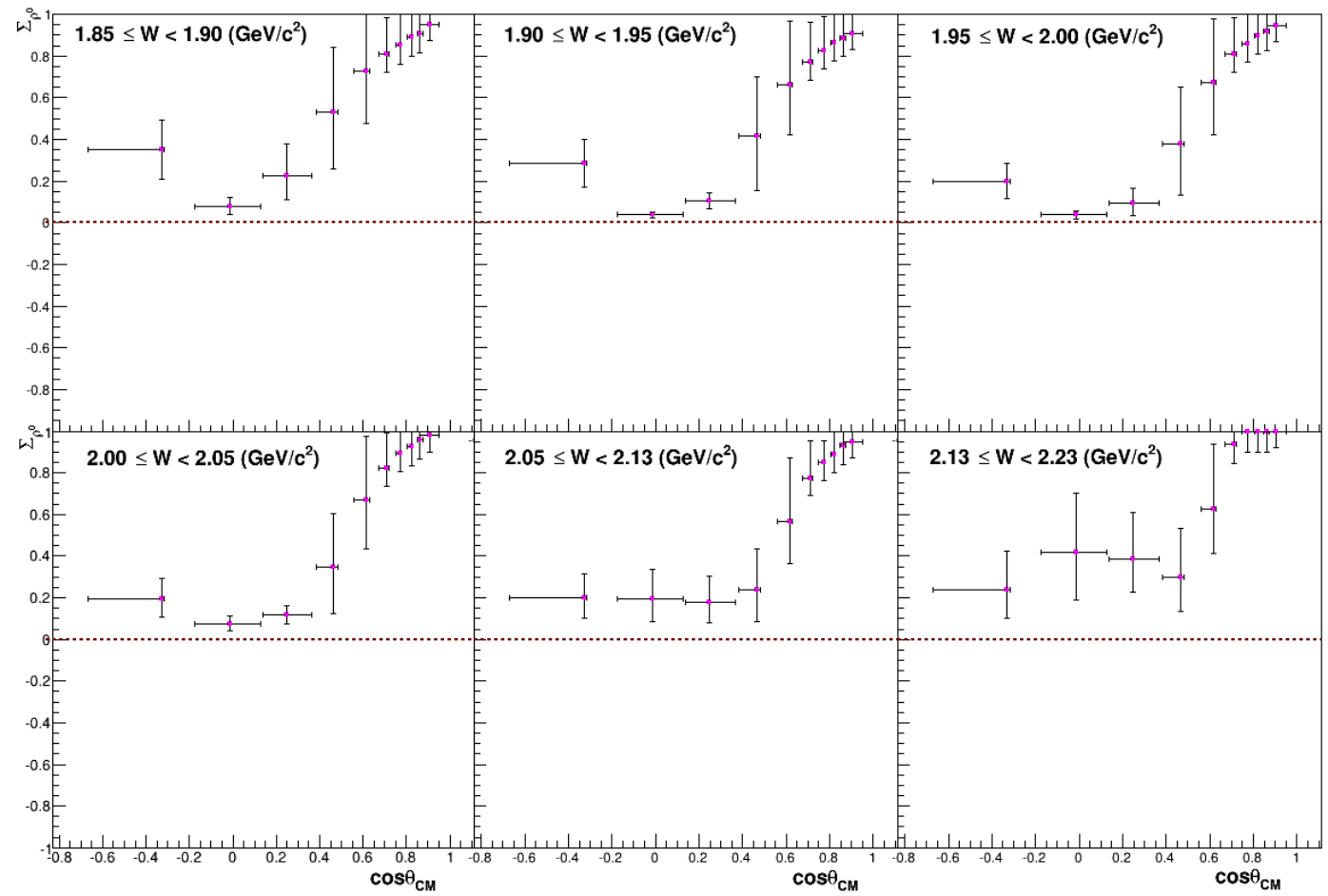

Figure 7.1: Beam asymmetry extracted values for $\rho^{0}$ as a function of $\cos \theta_{C M}$ for different $W$ regions. The error bars include the statistical uncertainty, the uncertainty associated with the signal background separation, and the error estimation from the fitting procedure.

The results from the 2-d procedure are shown in figure 7.1 for the $\rho^{0}$ meson. A strong diffractive dominance can be seen in the foward angle region where $\Sigma \approx 1$, whereas other processes other than the t-channel production mechanism are more dominant in the backward angle. There is no clear structure in any of the $W$ ranges and so the only way to test for specific resonance sensitivity is to compare with model predictions. However, the structure of the trend in the backward region does change relative to the $W$ region which might suggest that the different resonances involved affect the beam asymmetry differently. This energy dependence will be useful for aiding theoretical predictions.

Figure 7.2 shows the cross sections from an analysis with similar photon energy ranges. The beam asymmetries measured here follow a similar trend as the cross sections, but with more structure in the background region of the asymmetry trends at higher energies than for the equivalent cross sections. 

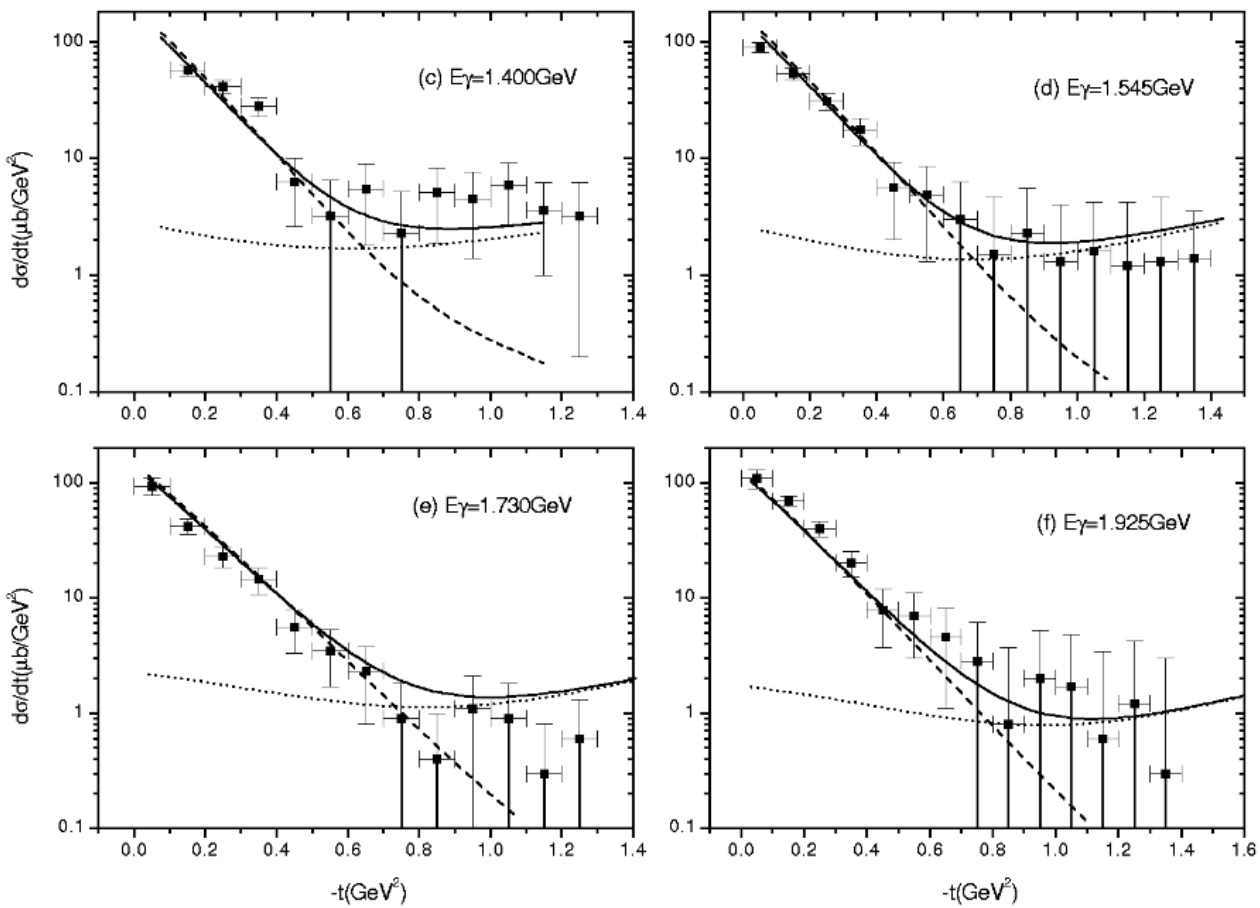

Figure 7.2: Cross sections extracted measured for $\rho^{0}$ a function of $-t$ for different $\mathrm{W}$ regions spanning 1.87-2.12 GeV [83]. The dotted line show model predictions from Zhao using the quark model [84].

The results for the process of extracting the separate beam asymmetry for the $f^{0}$ region at backward angles is shown in figure 7.3 using the 1-D fit procedure discussed above. The trends of these plots differ significantly from those of the $\rho^{0}$ in figure 7.1. The $f^{0}$ plots have a much more stable distributions with respect to $\cos \theta_{C M}$ and also to $W$. The difference between the $f^{0}$ and the $\rho^{0}$ beam asymmetries could prove significant and will undoubtedly be useful information when analysing the effects of intermediate resonances on the predicted values for these measurements. Cross sections result for the $f^{0}$ are shown in 7.4 but for an energy setting above this experiment. This helps to emphasise that the results measured for the $f^{0}$ here to indeed deviate from purely diffractive values as the beam asymmetries do not follow the near linear relationship seen in 7.4 .

In addition to these results we have opted to display the separate pion asymmetries as a function of the $M_{\pi^{+} \pi^{-}}$for different $\cos \theta_{C M}$ regions in order to investigate the differences between the region of the $\rho^{0}$ and the region of the $f^{0}$ and the other non- 


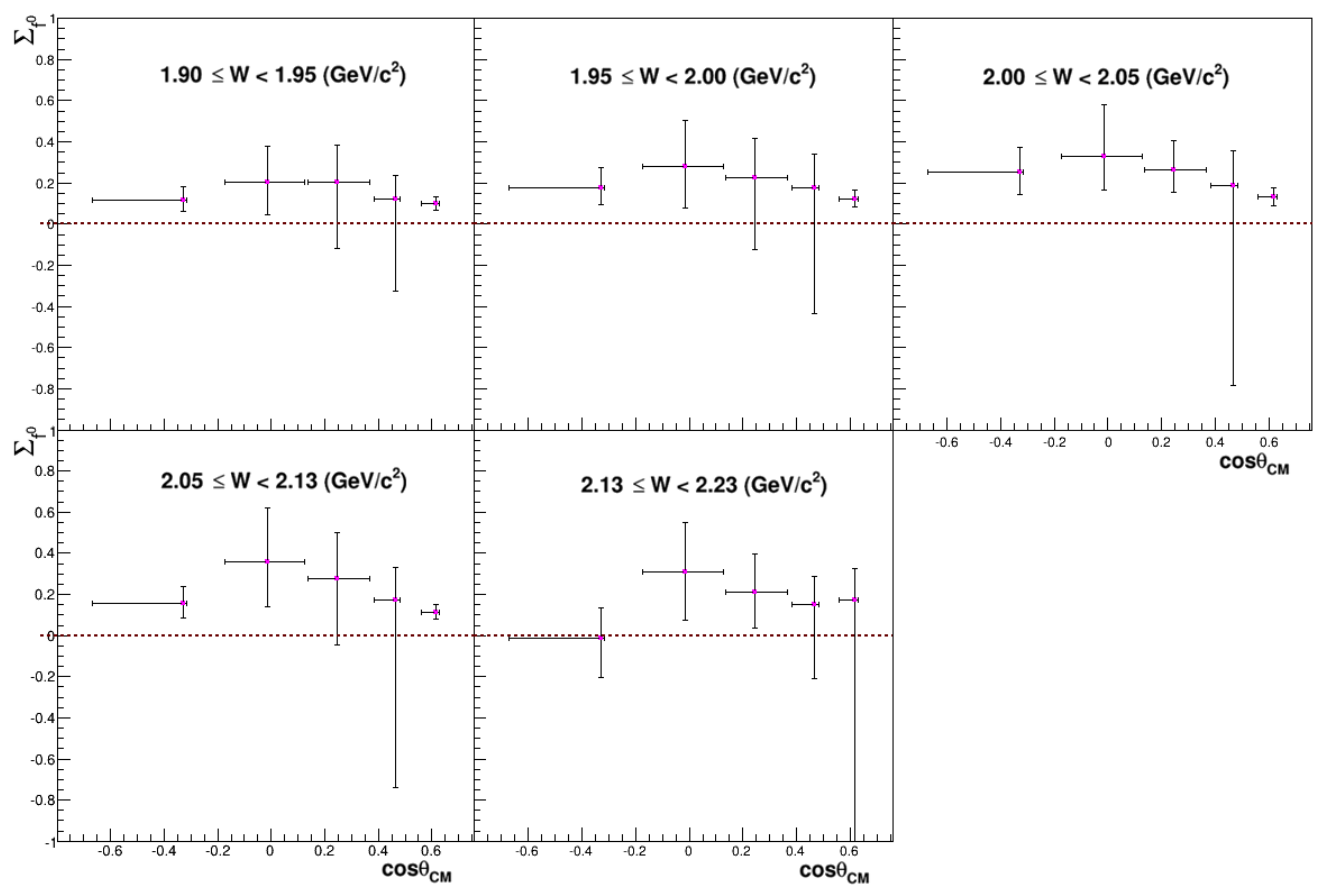

Figure 7.3: Beam asymmetry extracted values for $f^{0}$ as a function of $\cos \theta_{C M}$ for different $W$ regions. The error bars include the statistical uncertainty, the uncertainty associated with the signal background separation, and the error estimation from the fitting procedure.

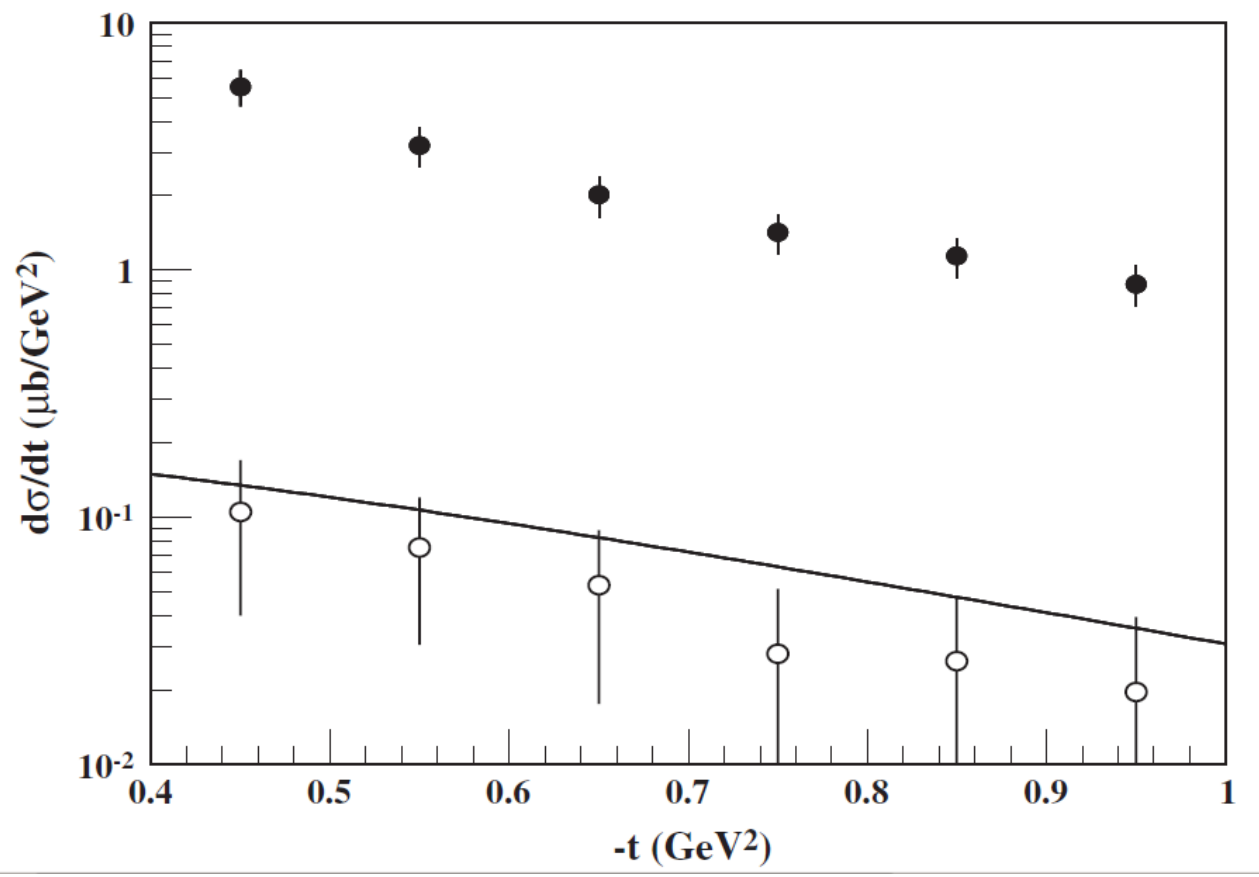

Figure 7.4: Cross sections extracted measured for $f^{0}$ a function of $-t$ for photon energy range $E_{\gamma}=3.0-3.8 \mathrm{GeV}[49]$. 


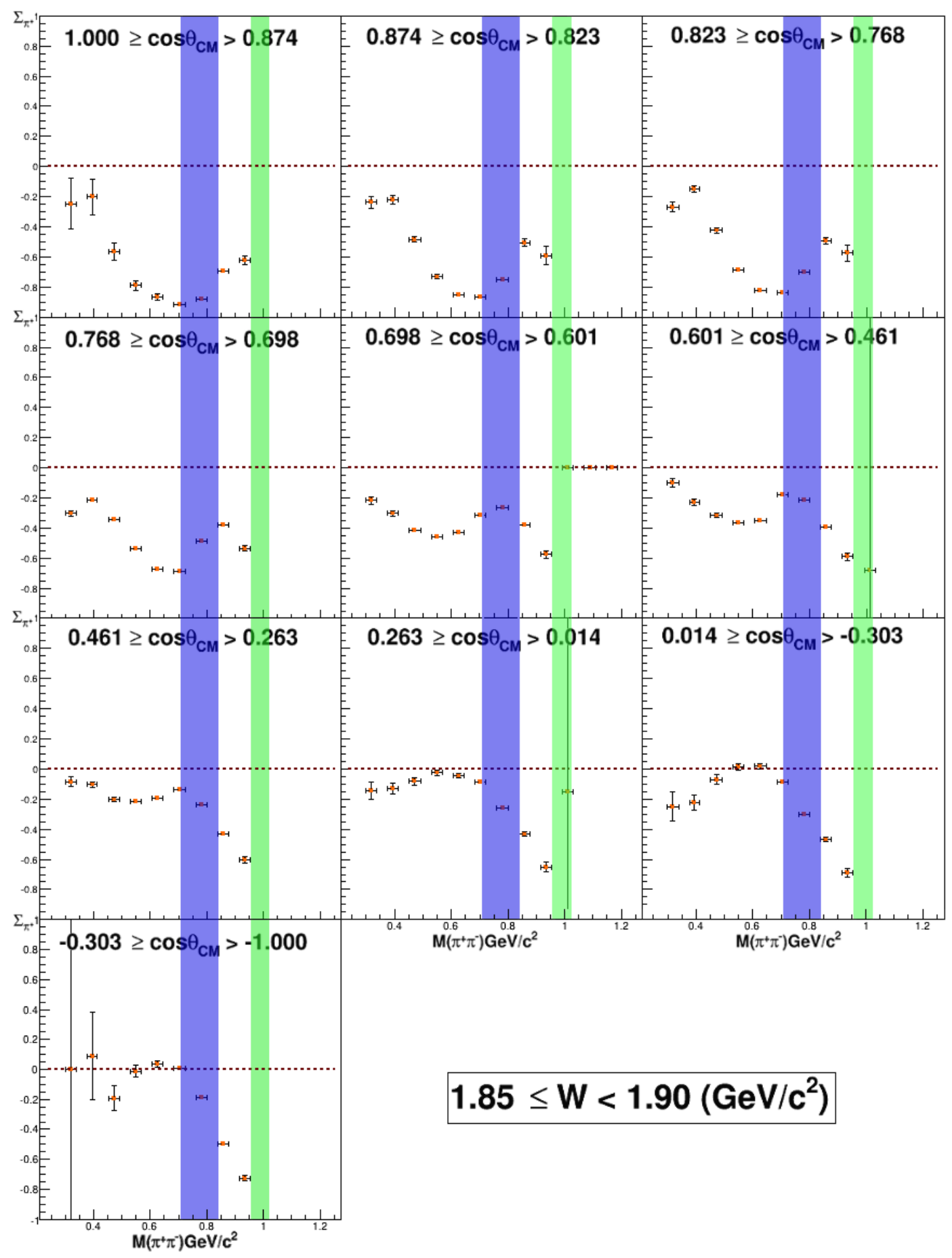

Figure 7.5: Beam asymmetry extracted values for the $\pi^{+}$as a function of $M\left(\pi^{+} \pi^{-}\right)$for different $\mathrm{W}$ region 1 . The error bars include the statistical uncertainty, the uncertainty associated with the signal background separation, and the error estimation from the fitting procedure. 


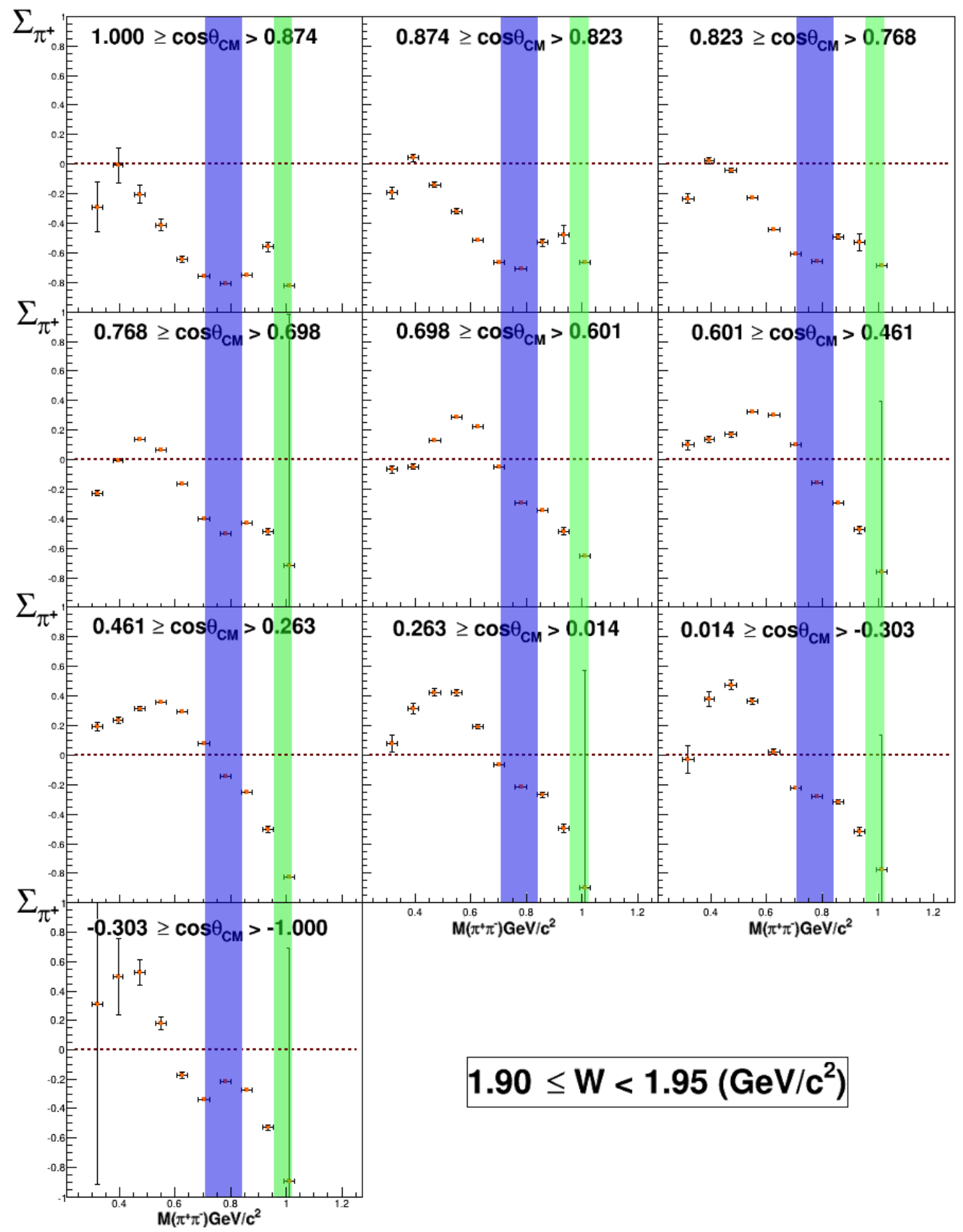

Figure 7.6: Beam asymmetry extracted values for the $\pi^{+}$as a function of $M\left(\pi^{+} \pi^{-}\right)$ for $\mathrm{W}$ region 2 . The error bars include the statistical uncertainty, the uncertainty associated with the signal background separation, and the error estimation from the fitting procedure. 


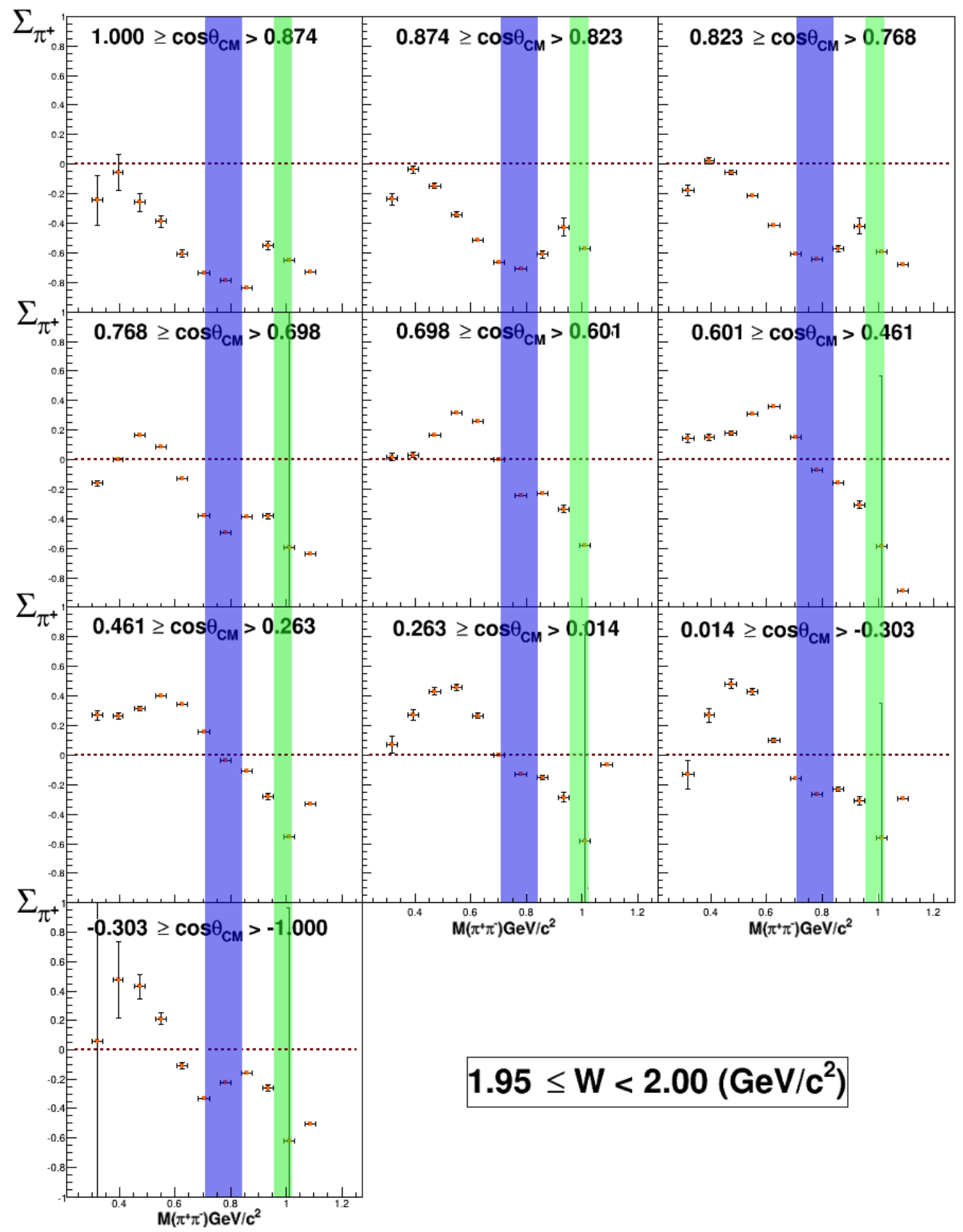

Figure 7.7: Beam asymmetry extracted values for the $\pi^{+}$as a function of $M\left(\pi^{+} \pi^{-}\right)$ for $\mathrm{W}$ region 3 . The error bars include the statistical uncertainty, the uncertainty associated with the signal background separation, and the error estimation from the fitting procedure. 


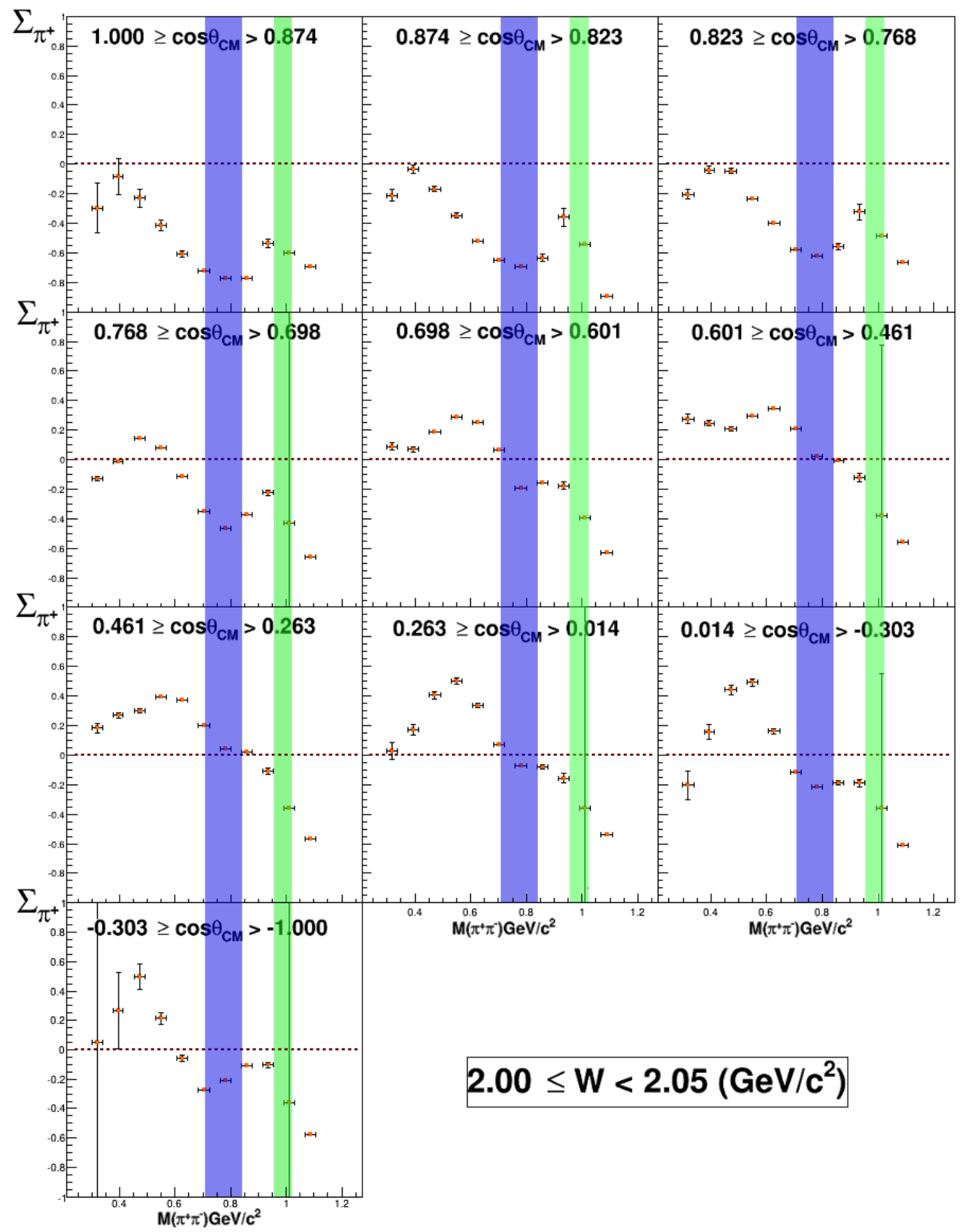

Figure 7.8: Beam asymmetry extracted values for the $\pi^{+}$as a function of $M\left(\pi^{+} \pi^{-}\right)$ for $\mathrm{W}$ region 4 . The error bars include the statistical uncertainty, the uncertainty associated with the signal background separation, and the error estimation from the fitting procedure. 


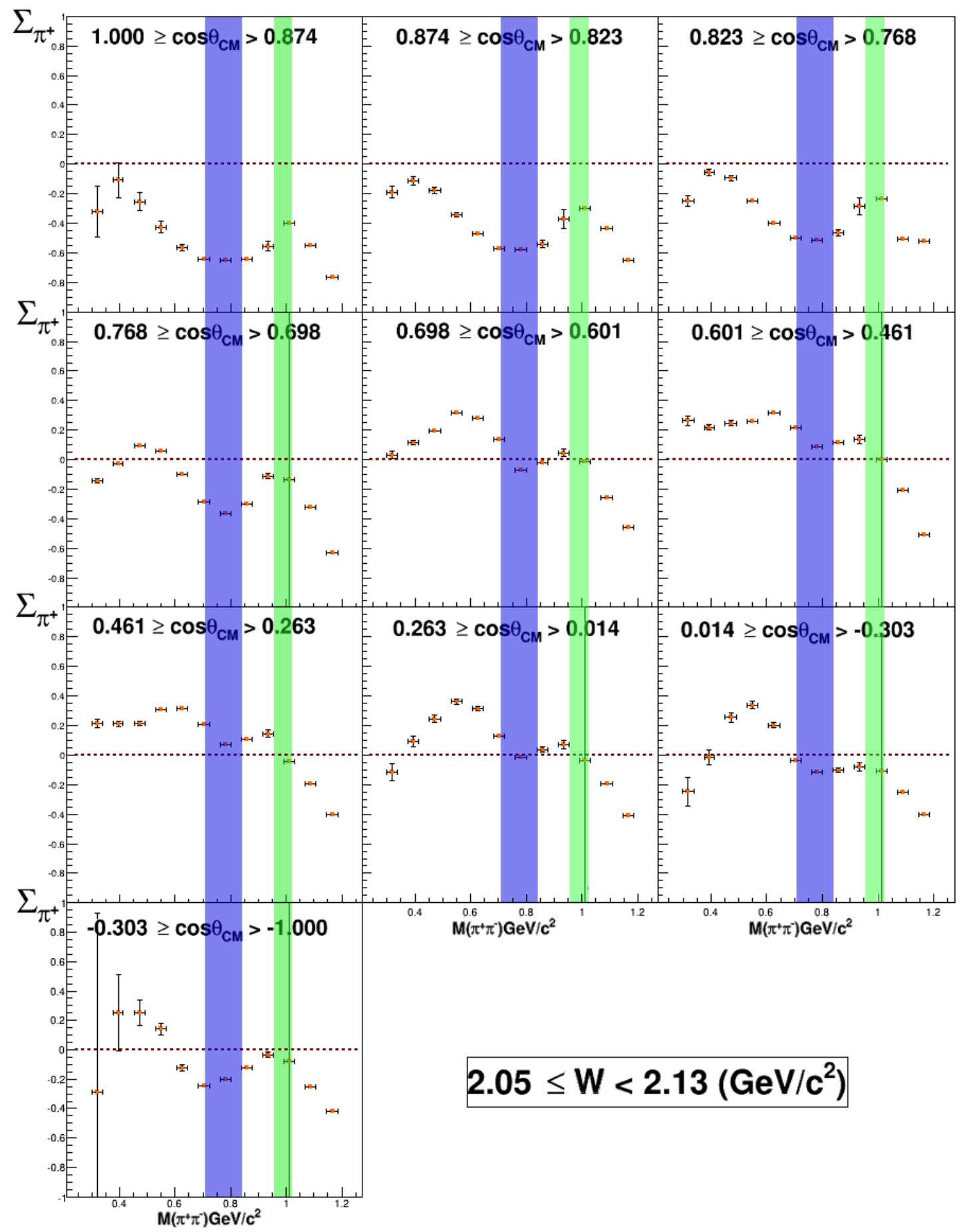

Figure 7.9: Beam asymmetry extracted values for the $\pi^{+}$as a function of $M\left(\pi^{+} \pi^{-}\right)$ for $\mathrm{W}$ region 5 . The error bars include the statistical uncertainty, the uncertainty associated with the signal background separation, and the error estimation from the fitting procedure. 


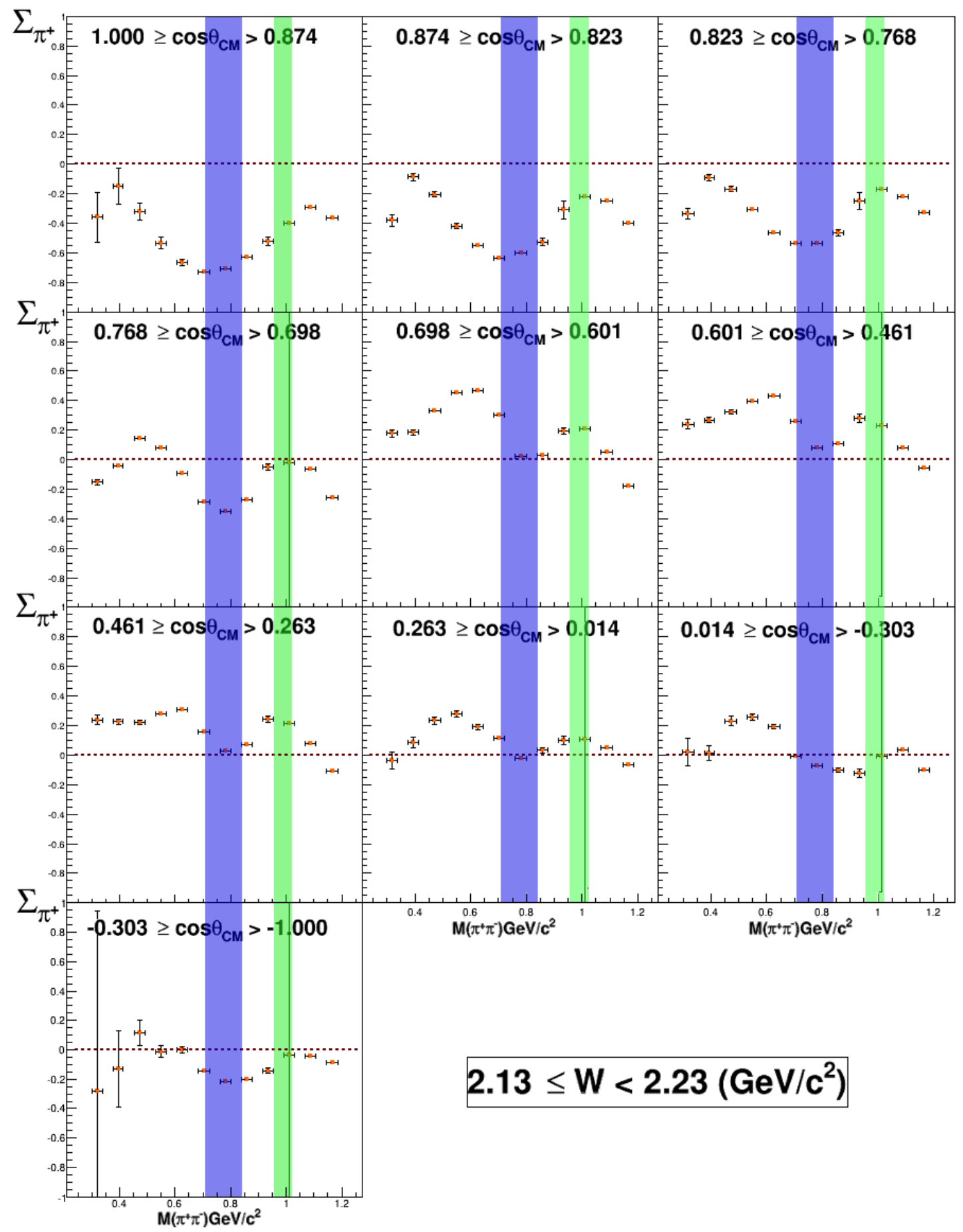

Figure 7.10: Beam asymmetry extracted values for the $\pi^{+}$as a function of $M\left(\pi^{+} \pi^{-}\right)$ for $\mathrm{W}$ region 6 . The error bars include the statistical uncertainty, the uncertainty associated with the signal background separation, and the error estimation from the fitting procedure. 


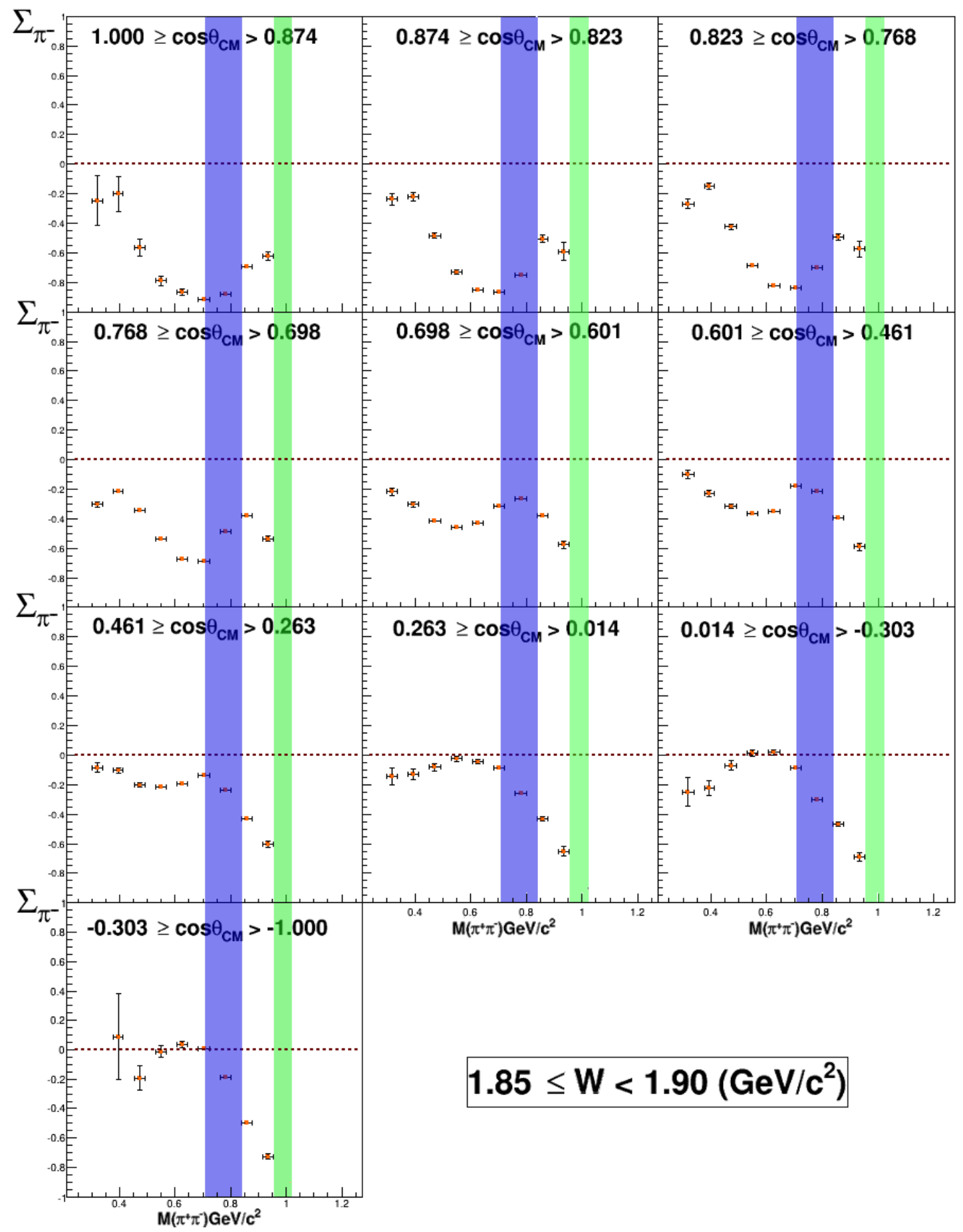

Figure 7.11: Beam asymmetry extracted values for the $\pi^{-}$as a function of $M\left(\pi^{+} \pi^{-}\right)$ for $\mathrm{W}$ region 1 . The error bars include the statistical uncertainty, the uncertainty associated with the signal background separation, and the error estimation from the fitting procedure. 


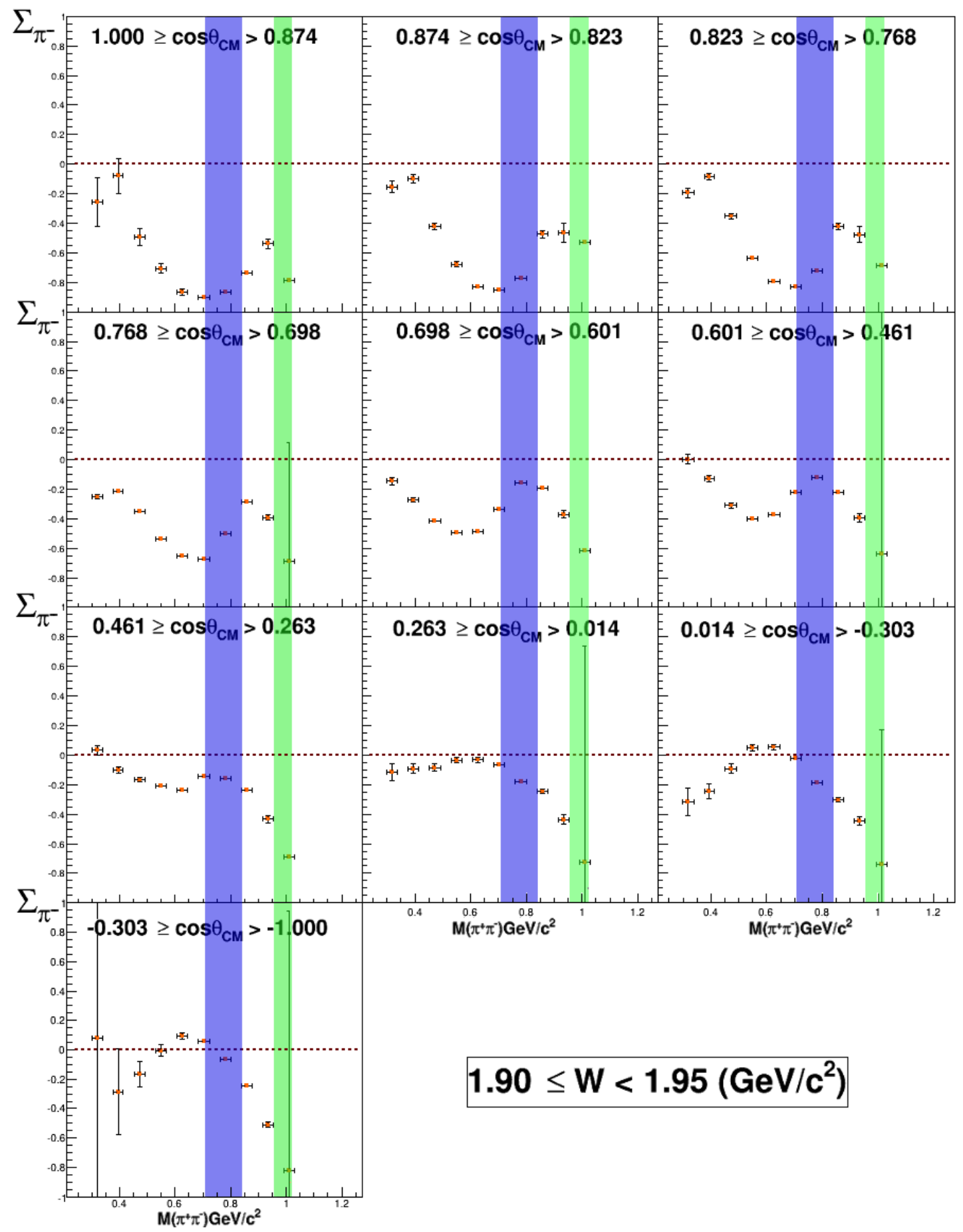

Figure 7.12: Beam asymmetry extracted values for the $\pi^{-}$as a function of $M\left(\pi^{+} \pi^{-}\right)$ for $\mathrm{W}$ region 2 . The error bars include the statistical uncertainty, the uncertainty associated with the signal background separation, and the error estimation from the fitting procedure. 


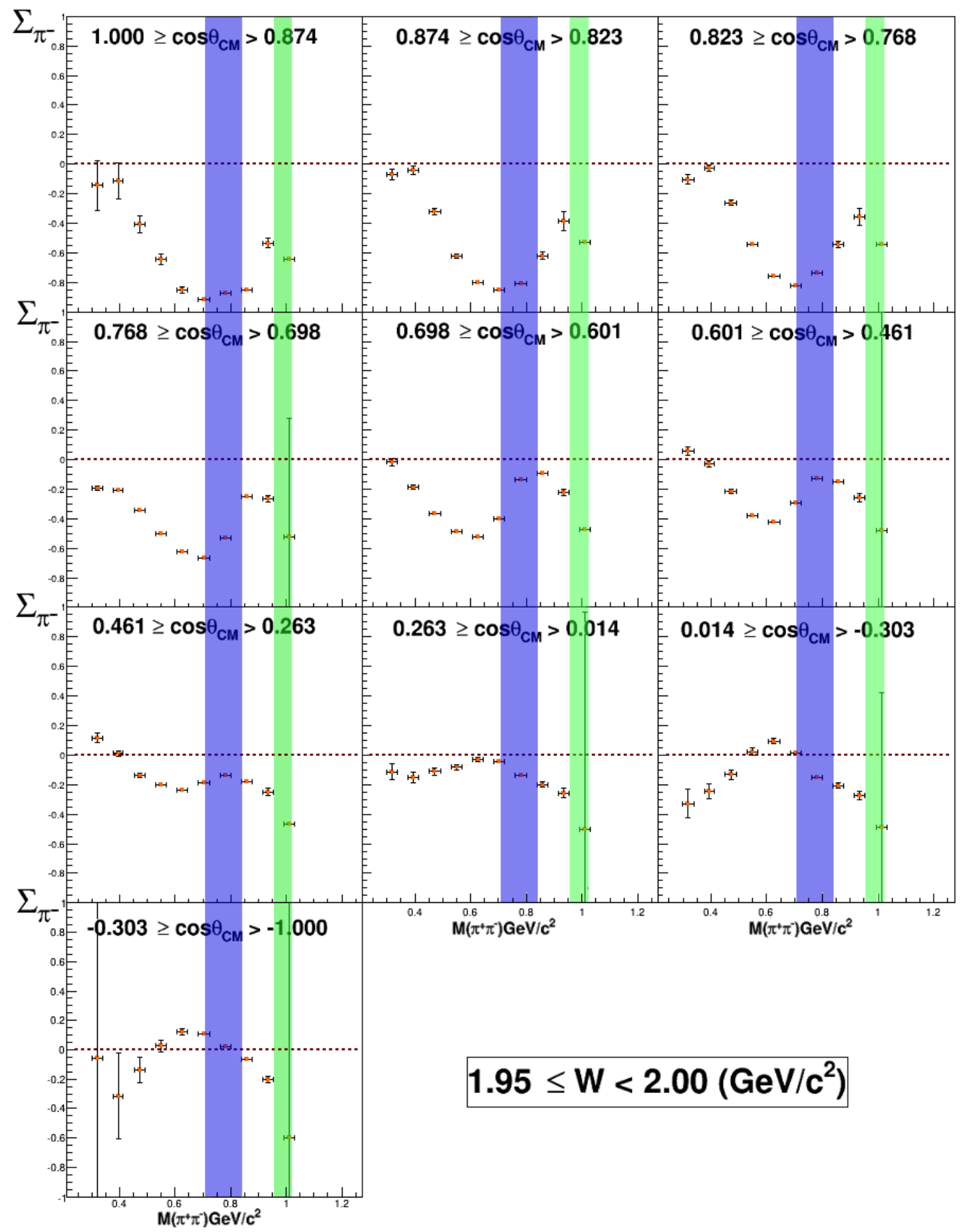

Figure 7.13: Beam asymmetry extracted values for the $\pi^{-}$as a function of $M\left(\pi^{+} \pi^{-}\right)$ for $\mathrm{W}$ region 3 . The error bars include the statistical uncertainty, the uncertainty associated with the signal background separation, and the error estimation from the fitting procedure. 


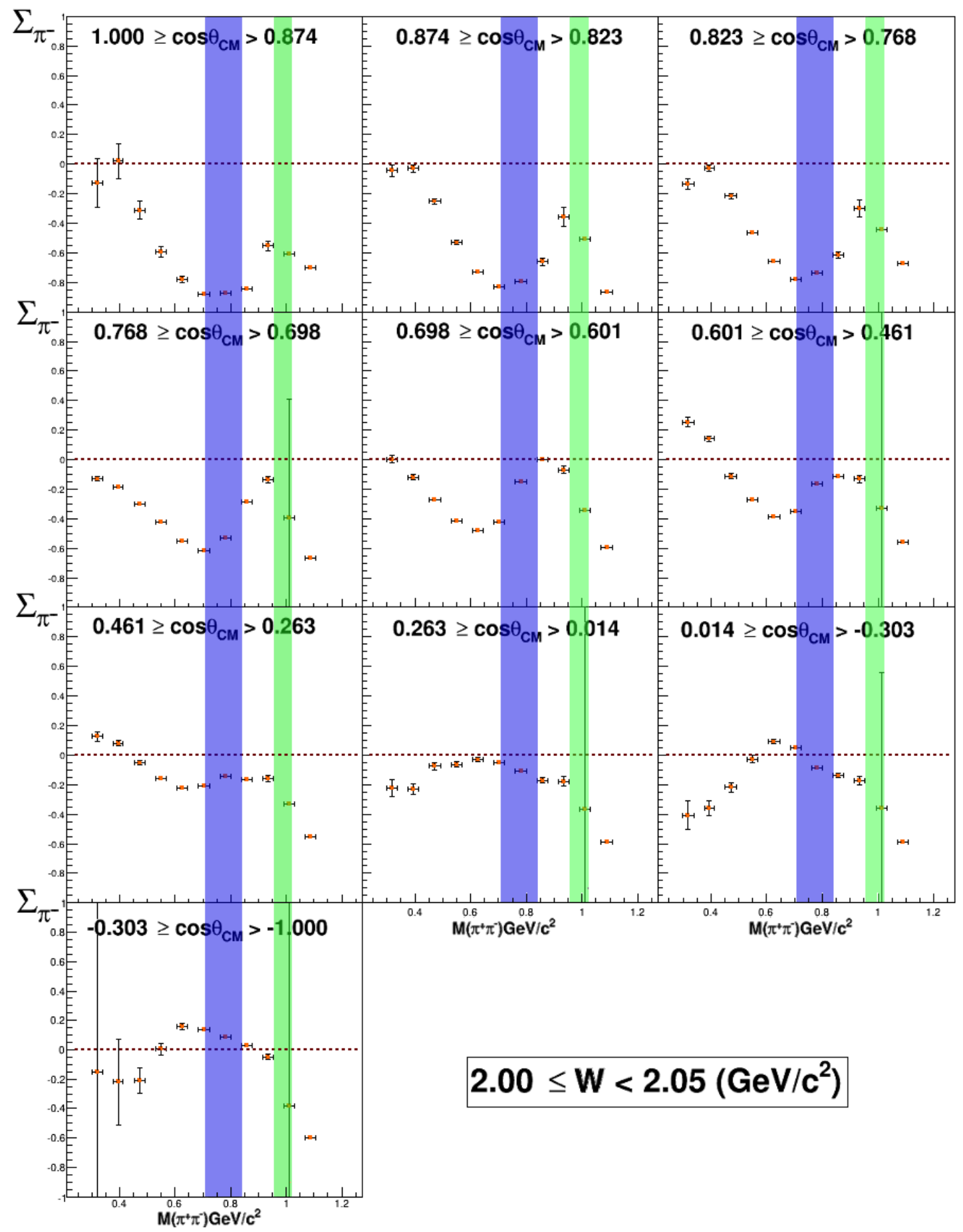

Figure 7.14: Beam asymmetry extracted values for the $\pi^{-}$as a function of $M\left(\pi^{+} \pi^{-}\right)$ for $\mathrm{W}$ region 4 . The error bars include the statistical uncertainty, the uncertainty associated with the signal background separation, and the error estimation from the fitting procedure. 


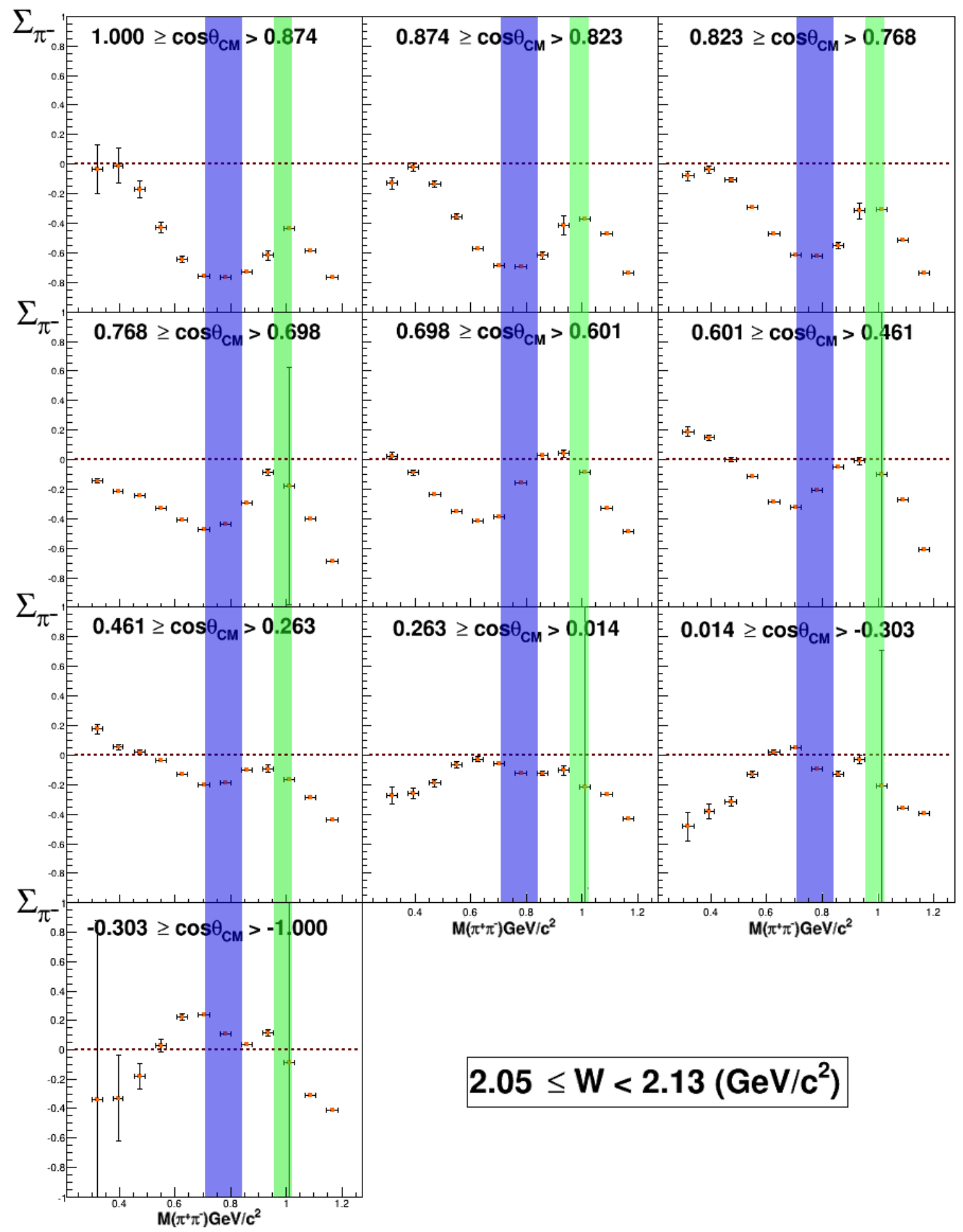

Figure 7.15: Beam asymmetry extracted values for the $\pi^{-}$as a function of $M\left(\pi^{+} \pi^{-}\right)$ for $\mathrm{W}$ region 5 . The error bars include the statistical uncertainty, the uncertainty associated with the signal background separation, and the error estimation from the fitting procedure. 


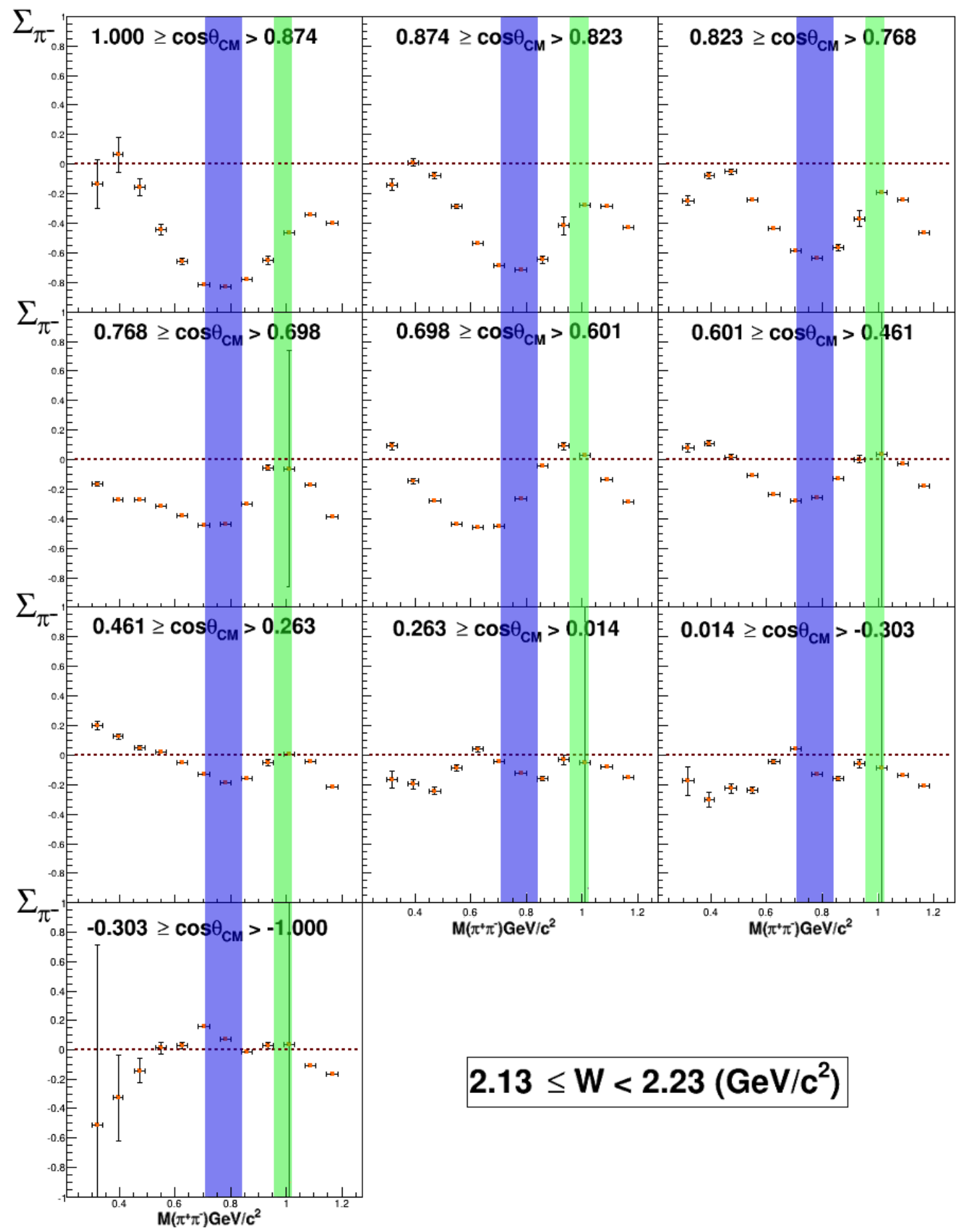

Figure 7.16: Beam asymmetry extracted values for the $\pi^{-}$as a function of $M\left(\pi^{+} \pi^{-}\right)$ for $\mathrm{W}$ region 6 . The error bars include the statistical uncertainty, the uncertainty associated with the signal background separation, and the error estimation from the fitting procedure. 
resonant background regions.

These high statistic results for the single pions are shown for all the $W$ ranges spanning the dataset and in separate $\cos \theta_{C M}$ bins first for the $\pi^{+}$in figures 7.5 to 7.10 and then for the $\pi^{-}$in figures 7.11 to 7.15 . It is clear in the backward angles that the $f^{0}$ meson and the $\rho^{0}$ meson are sensitive to the beam asymmetry in different ways and suggests that a better separation of the processes using a partial wave analysis would be useful in distinguishing different intermediate resonant behaviour for these two meson resonances, similar to the study done at INFN, Genova with the CLAS collaboration for the two pion extraction of SDMEs available without a polarized beam and the cross section at energies further from the threshold energy used in this analysis (3.$3.8 \mathrm{GeV}$ ) [50]. A great deal of structure is seen in figures 7.5 to 7.15 , both with respect to the $\cos \theta_{C M}$ regions and the different $W$ regions. The trends for both the positive and negative pions are often similar but there are some remarkable differences. For example, a major difference can be seen in figure 7.10 and figure 7.16, showing $\Sigma$ in the $W$ region 2.13 to $2.23 \mathrm{GeV} / \mathrm{c}^{2}$ for the positive and negative pion, respectively. In the foward region, the first three plots, the pion asymmetires have a similar trend with a slight difference in terms of magnitude. But in the backward region, especially the $\cos \theta_{C M}$ region 0.601 to 0.601 , there is a positive signal seen in the plot for the positive pion at about $0.6 \mathrm{GeV} / \mathrm{c}^{2}$ but in the same plot for the negative pion a negative signal is seen with a similar magnitude. On both of these plots after $0.8 \mathrm{GeV} / \mathrm{c} c^{2}$ they follow the same trend again. Differences like these suggest that the decay products of photoproduced mesons can be affected by intermediate states and can be affected differently. These different effects are then seen in the polarization observables we measure in our experiments. This could prove to be espeically useful for reaction channels which are difficult to analyse, such as the $\rho^{0}$ meson. 


\subsection{Summary}

In this chapter the results obtained from this analysis have been shown and discussed.

The discussion was aided by the previously measured cross sections for the $\rho^{0}$ meson and the $f^{0}$ meson. The comparison to these cross sections emphasised the sensitivity seen in the beam asymmetry distributions to resonant processes as they deviate from a purely diffractive picture. Also, strong structures were observed in the asymmetry measuremants for the pions. These were mainly due to the different contributions from the $\rho^{0}$ meson and the $f^{0}$ meson, but differences were observed in the trends of the distribution relative to the different $\cos \theta_{C M}$ regions and the $W$ regions. In addition to this, differences were observed and discussed between the distributions of the different pions. How these results fit into the wider context and what they might mean for hadron spectroscopy is discussed in the following chapter. 


\section{Chapter 8}

\section{Conclusion and Discussion}

Beam asymmetry measurements for the $\rho^{0}$ meson, the $f^{0}$ meson, and the detected pions for the reaction channel $\gamma p \rightarrow p \pi^{+} \pi^{-}$have been measured as part of the $N^{*}$ Program at JLab. The g8b experiment was the first experiment to use linearly polarized. Due to the $4 \pi$ coverage of the CLAS detector many multi-final state channels can be detected and so analyses using the g8b data have been completed for other channels, such as $N \pi, N \pi \pi, N \eta, K \Lambda$ and so on. This has resulted in many first measurements for polarization observables and where measurements were already existent, the larger statistics for the $\mathrm{g} 8 \mathrm{~b}$ experiment has increased the precision of these results.

The measurement of the beam asymmetries for the $\rho^{0}$ are the first results in the resonant region. While they show the characterstic diffractive nature of the $\rho^{0}$ in the foward angle region, they also present interesting characterstics in the backward angle region where resonant mechanisms are seen to contribute by the strong deviation from $\Sigma \sim 1$. It will be useful if model predictions can be made in the future so that the results presented here can be compared with different models with varying degrees of resonant contributions. The best case scenario would be explicit sensitivity to the inclusion and exclusion of certain resonances in these models, but even if this is not the case, these results can help develop the models by applying phenomological restrictions.

The $f^{0}$ measurements are the first beam asymmetry results for this meson in any energy region. The distributions have some meaningful structure to them which also 
varies with respect to the different $\mathrm{W}$ regions. As discussed above for the $\rho^{0}$, model predictions are needed to truly appreciate how meaningful these results are in terms of being able to establish or dismiss the existence of resonances.

And finally, the beam asymmetries extracted for the pions show intricate structures with sensitivity to the $\rho^{0}$ and the $f^{0}$. The aim is to use these high-statistic results to aid future partial wave analyses of this reaction channel. As these analyses move forward in a more coherent, community-driven manner, results like these can be more easily implemented and used in a wider range of studies. An example of one of these developments is the partial wave analysis software framework AmpTools [124]. AmpTools will allow individuals to undertake partial wave analysis with well-developed community software, rather than having to develop and implement their own. Results like the beam asymmetries for the pions extracted in this analysis will be vital to make software like this as accurate and useful as possible.

Independent of model comparisons and partial wave analysis developments, this specific analysis can be developed further to extract other polarization observables and explicit measurements for the individual SDMEs. In order to do this, a full acceptance correction study needs to be undertaken. Work on this has already begun and when complete it will result in further first measurements for this reaction channel.

\subsection{Summary}

High statistic, precision measurements of the beam asymmetries for the reaction channel $\gamma p \rightarrow p \pi^{+} \pi^{-}$have been measured with a focus on the intermediate mesonic states $\rho^{0}$ and $f^{0}$. This has given an insight into the transition mechanisms involved in this reaction channel, but the development of model predictions and a partial wave analysis are required to attain a detailed understanding of these mechanisms and the strength of their relative contributions. Many large scale collaborative efforts are being made on both fronts and hopefully it will not be long before measurements presented here, and many others like them, will be used in a decisive manner to help characterise the 
spectra of nucleons and establish which resonances exist, and which do not. 


\section{Appendix A}

\section{Invariant Mass Plots and Fits}

Plots similar to figure 6.37 shown for the all the invariant mass plots divided into kinematic regions as described in section 6.2.1. 


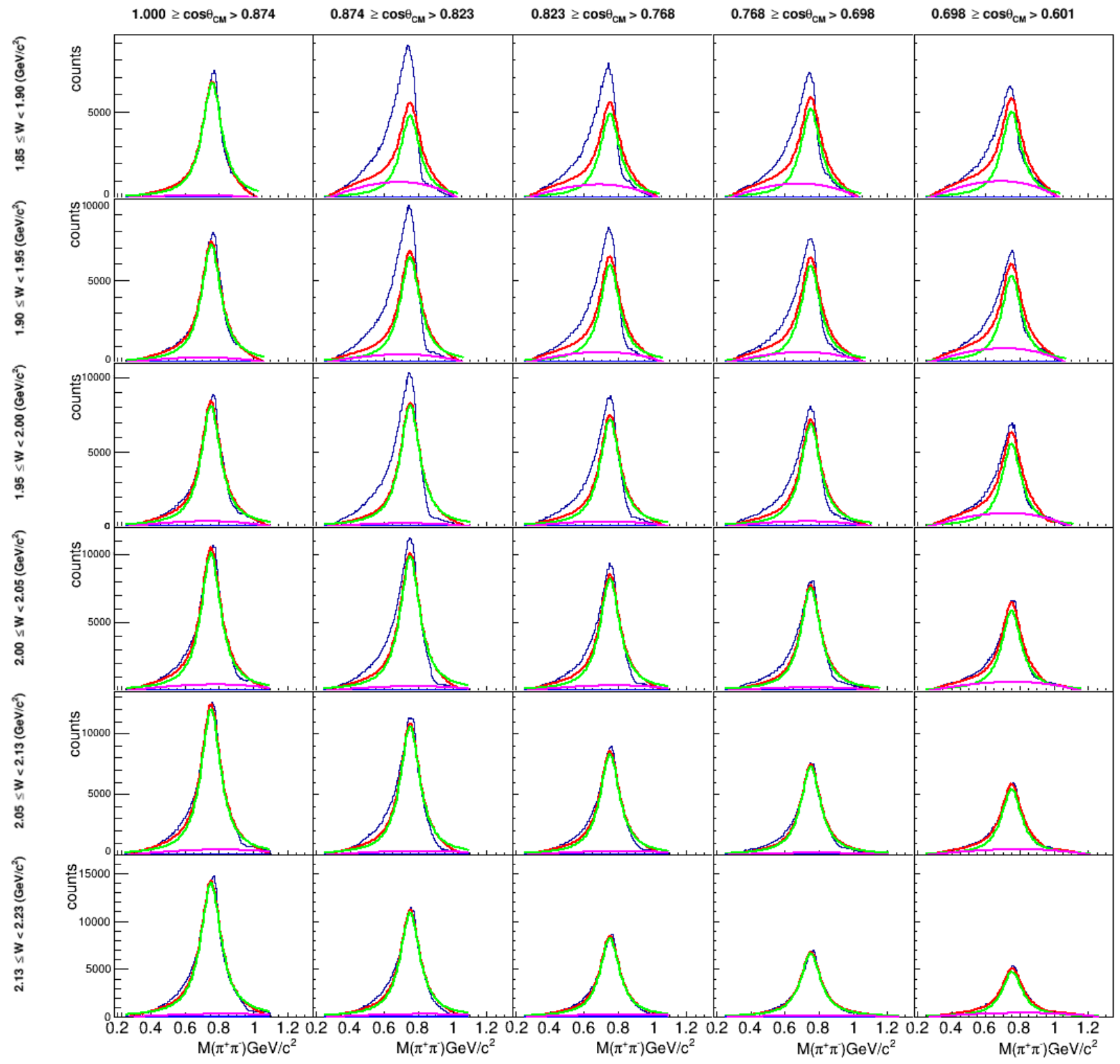

Figure A.1: The fit described in section 6.3 for each kinematic region for the forward polar angle region. Green represents the region of $\rho^{0}$, blue the $f^{0}$, magenta the background processes. 


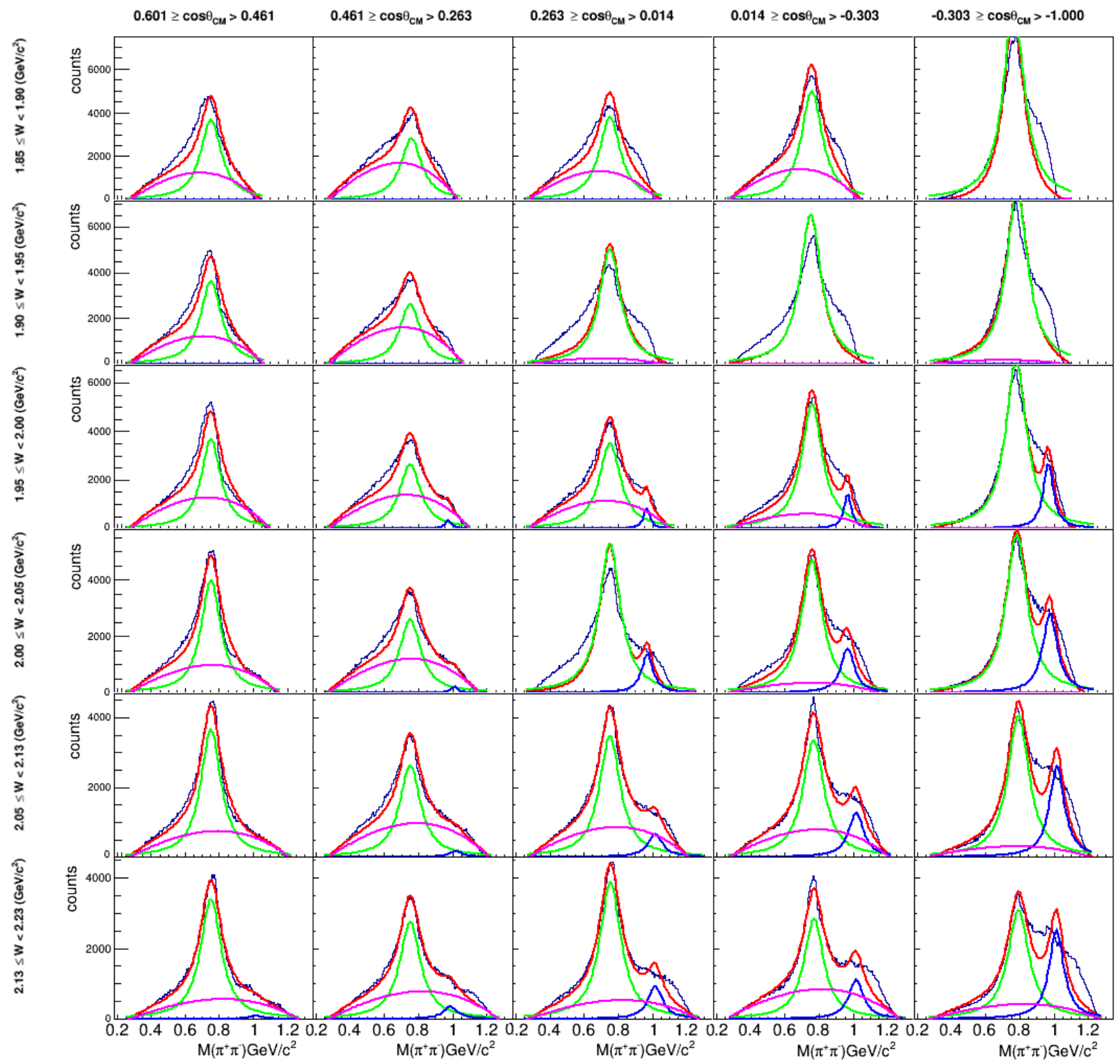

Figure A.2: The fit described in section 6.3 for each kinematic region for the backward polar angle region. Green represents the region of $\rho^{0}$, blue the $f^{0}$, magenta the background processes. 


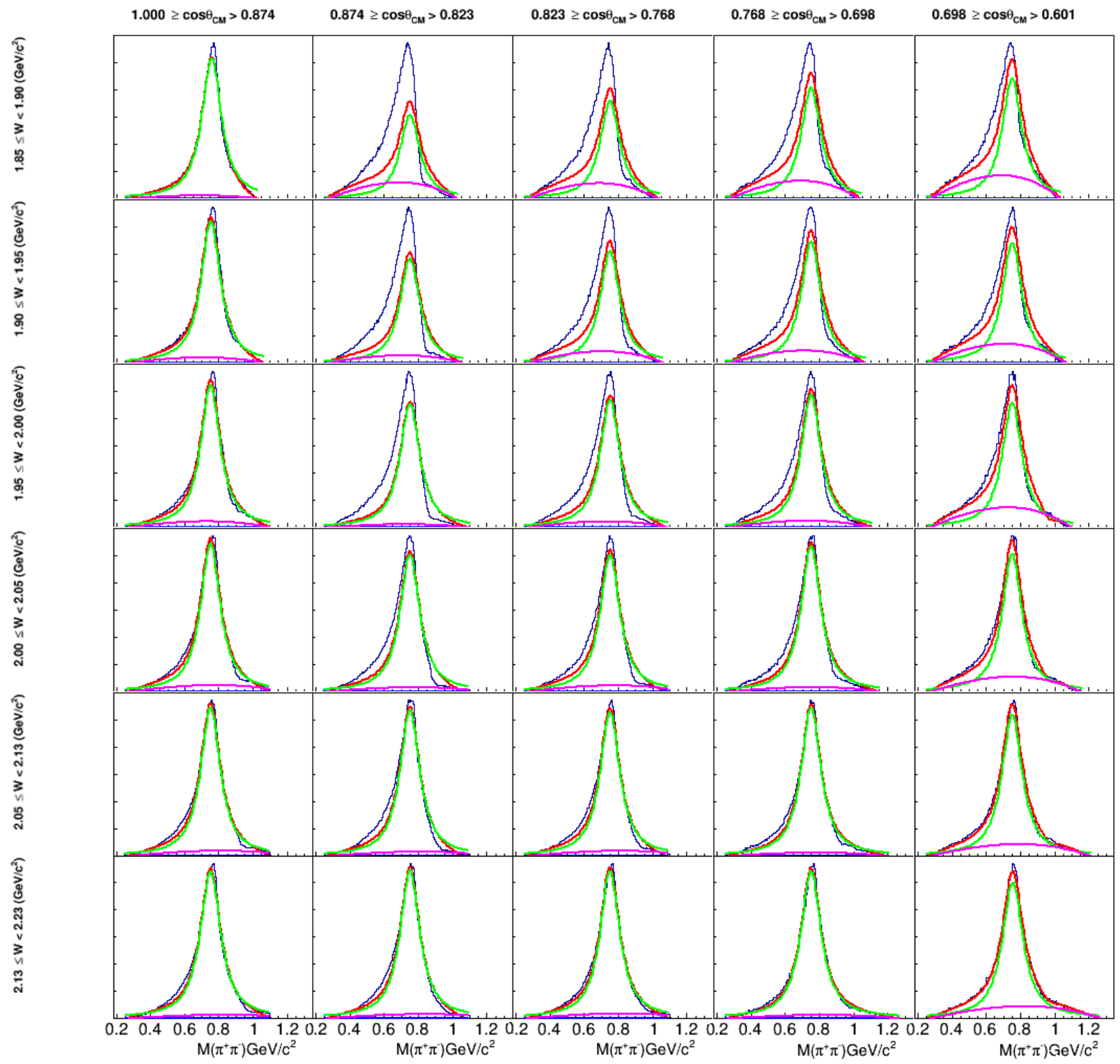

Figure A.3: The fit described in section 6.3 for each kinematic region for the forward polar angle region. Green represents the region of $\rho^{0}$, blue the $f^{0}$, magenta the background processes. Same as above in figure A.1 but scaled for clarity. 


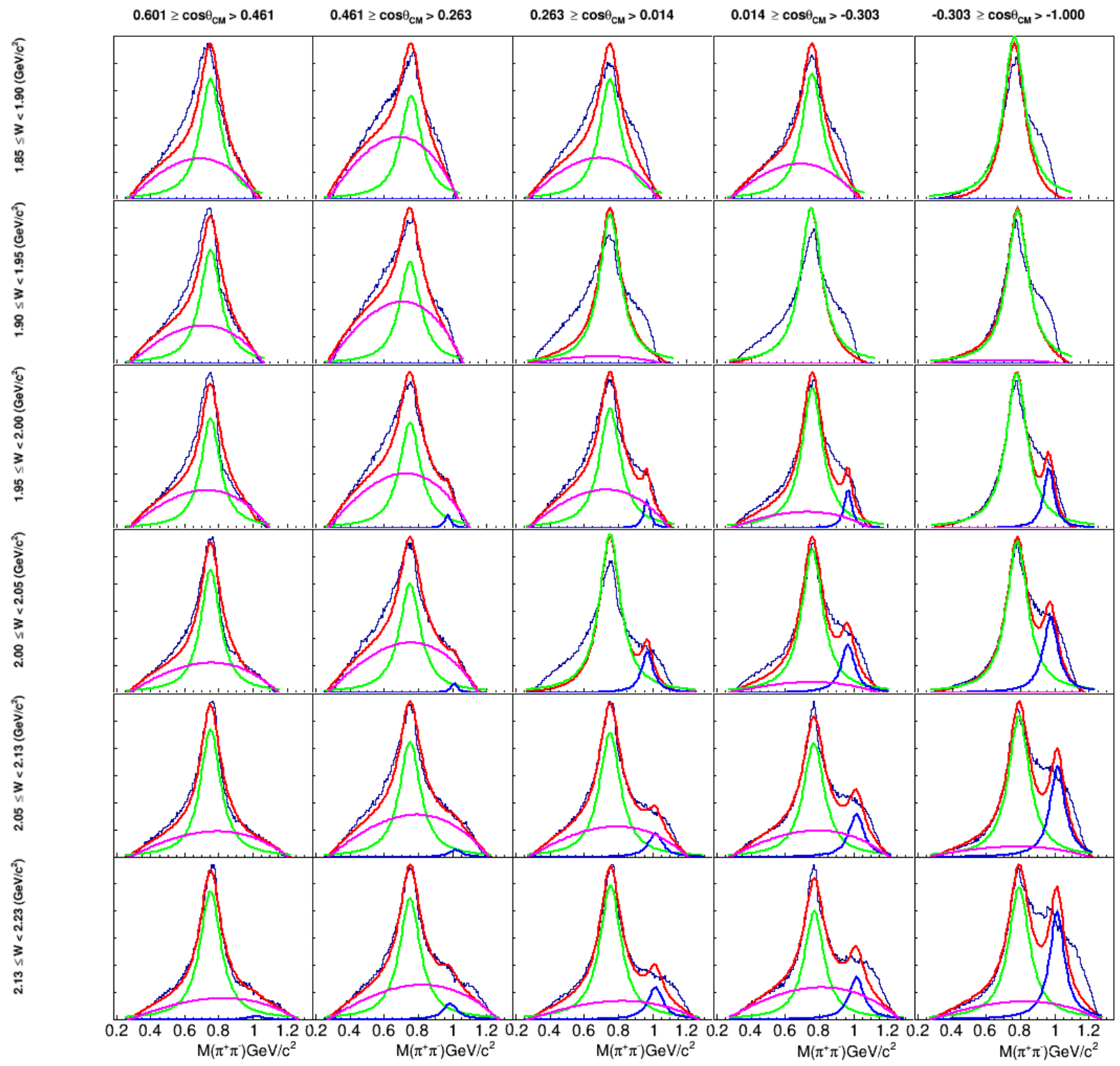

Figure A.4: The fit described in section 6.3 for each kinematic region for the backward polar angle region. Green represents the region of $\rho^{0}$, blue the $f^{0}$, magenta the background processes. Same as above in figure A.2 but scaled for clarity. 


\section{Appendix B}

\section{Asymmetry Fits}

The asymmetry distribution fits used to extract the beam asymmetry values shown in chapter 7 are shown here. First the two dimensional fits for the $\rho^{0}$ are shown in figure B.1 and then the one dimensional fits for the $f^{0}$ are shown in figure B.2. Figures B.4 to B.7 show the fits for the $\pi^{-}$in one $\mathrm{W}$ region and similarly for the $\pi^{+}$in figures B.8 to B.11. 


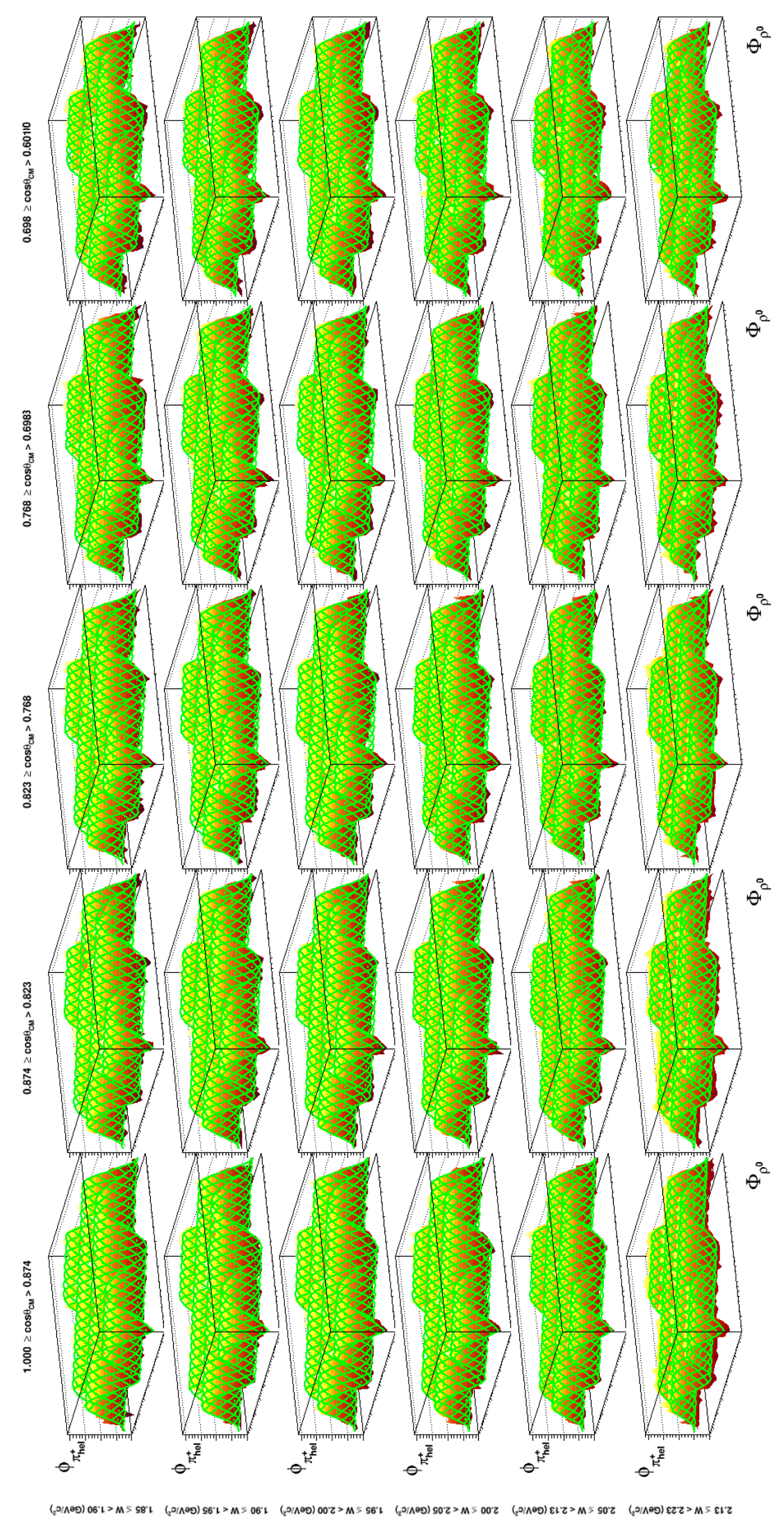

Figure B.1: The fit described in section 6.4 for each kinematic region for the forward polar angle region for extracting $\Sigma_{\rho^{0}}$. 


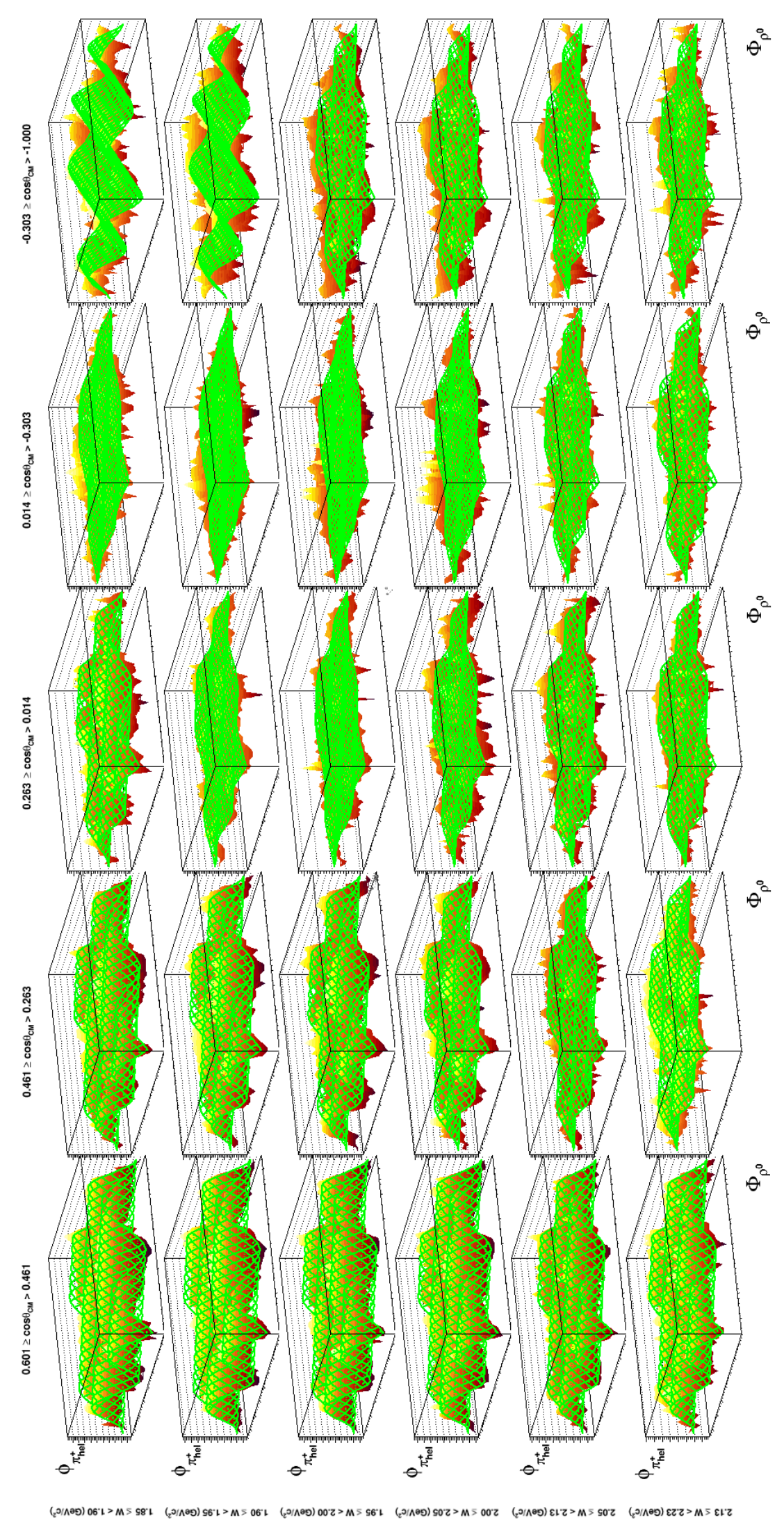

Figure B.2: The fit described in section 6.4 for each kinematic region for the backward polar angle region for extracting $\Sigma_{\rho^{0}}$. 


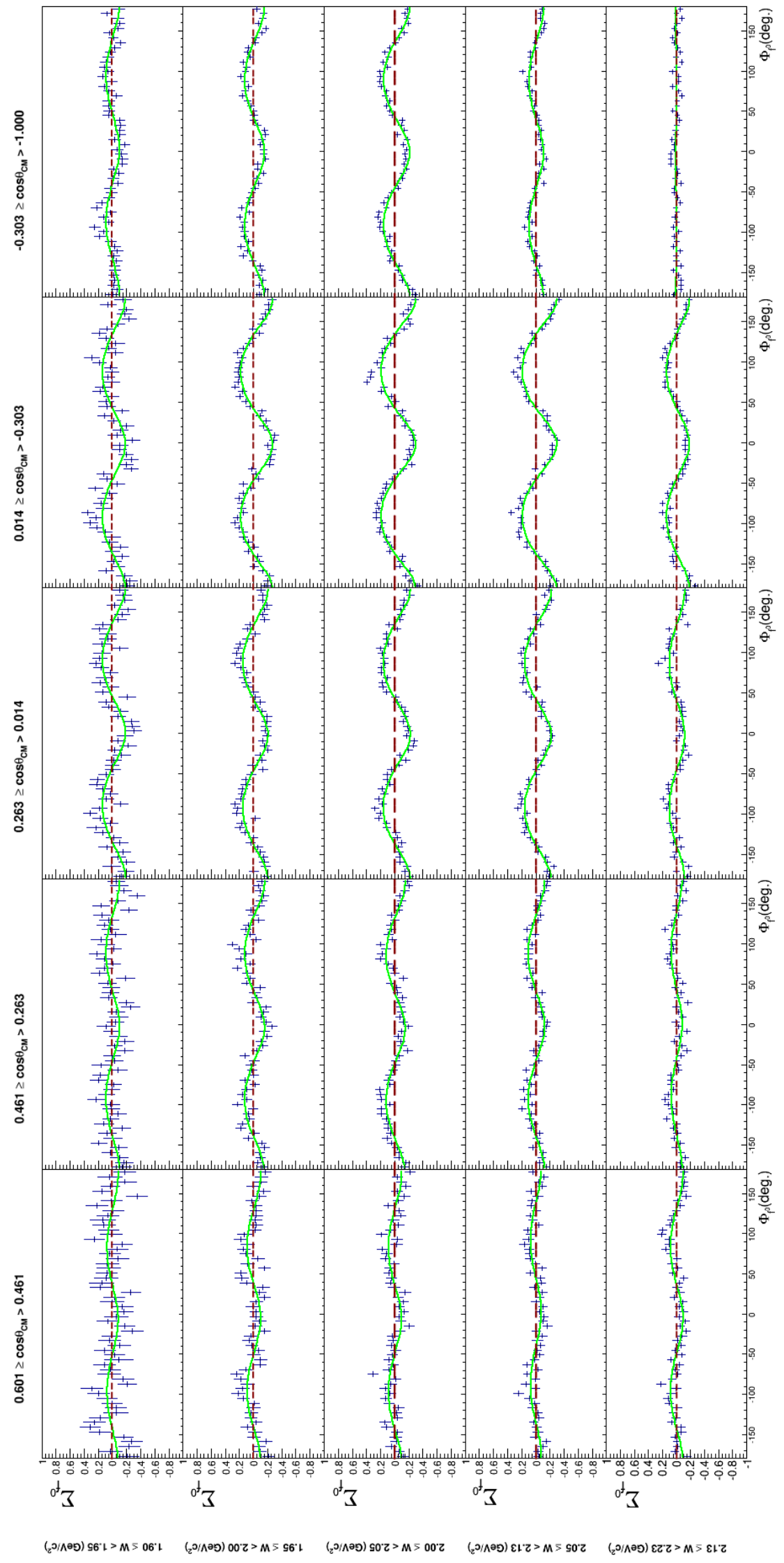

Figure B.3: The fit described in section 6.5 .1 for each kinematic region for extracting $\Sigma_{f^{0}}$. 


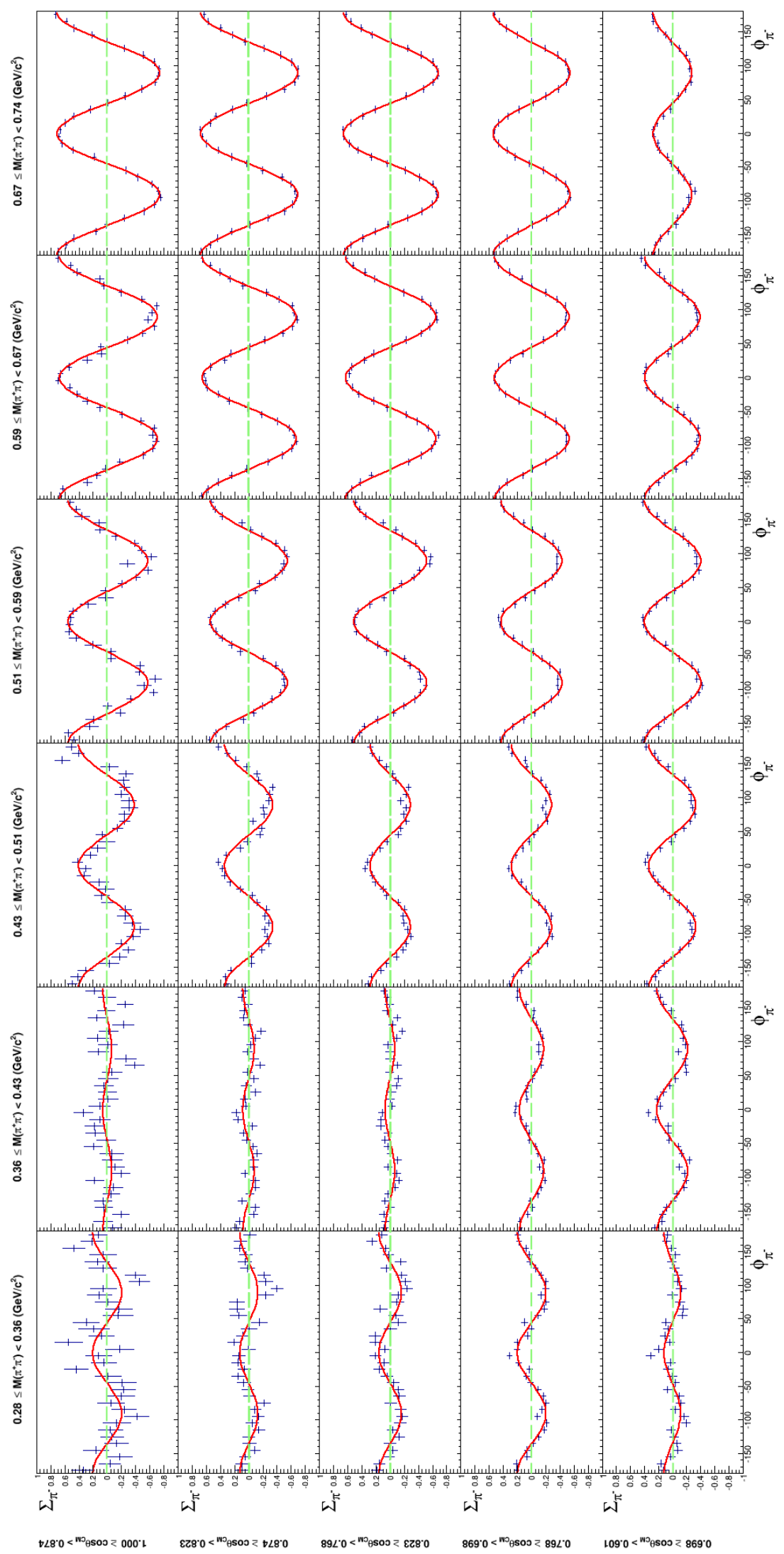

Figure B.4: The fit described in section 6.5 .1 for each kinematic region for extracting $\Sigma_{\pi^{-}}$for the second $\mathrm{W}$ region as defined in 6.2 .1 for the first half of the invariant mass distribution of the two pions in the forward angle region. 


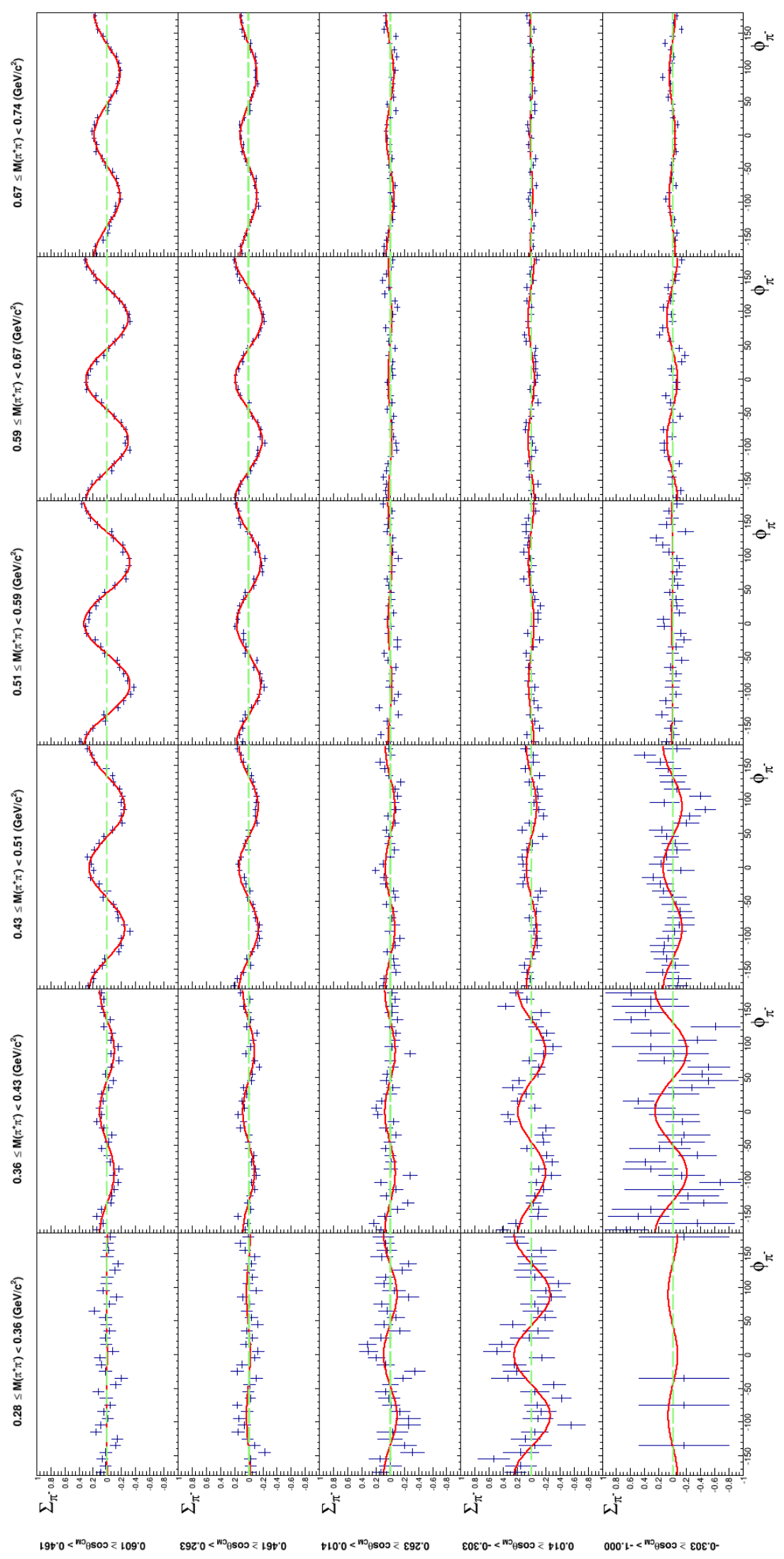

Figure B.5: The fit described in section 6.5 .1 for each kinematic region for extracting $\Sigma_{\pi^{-}}$for the second $\mathrm{W}$ region as defined in 6.2 .1 for the first half of the invariant mass distribution of the two pions in the backward angle region. 


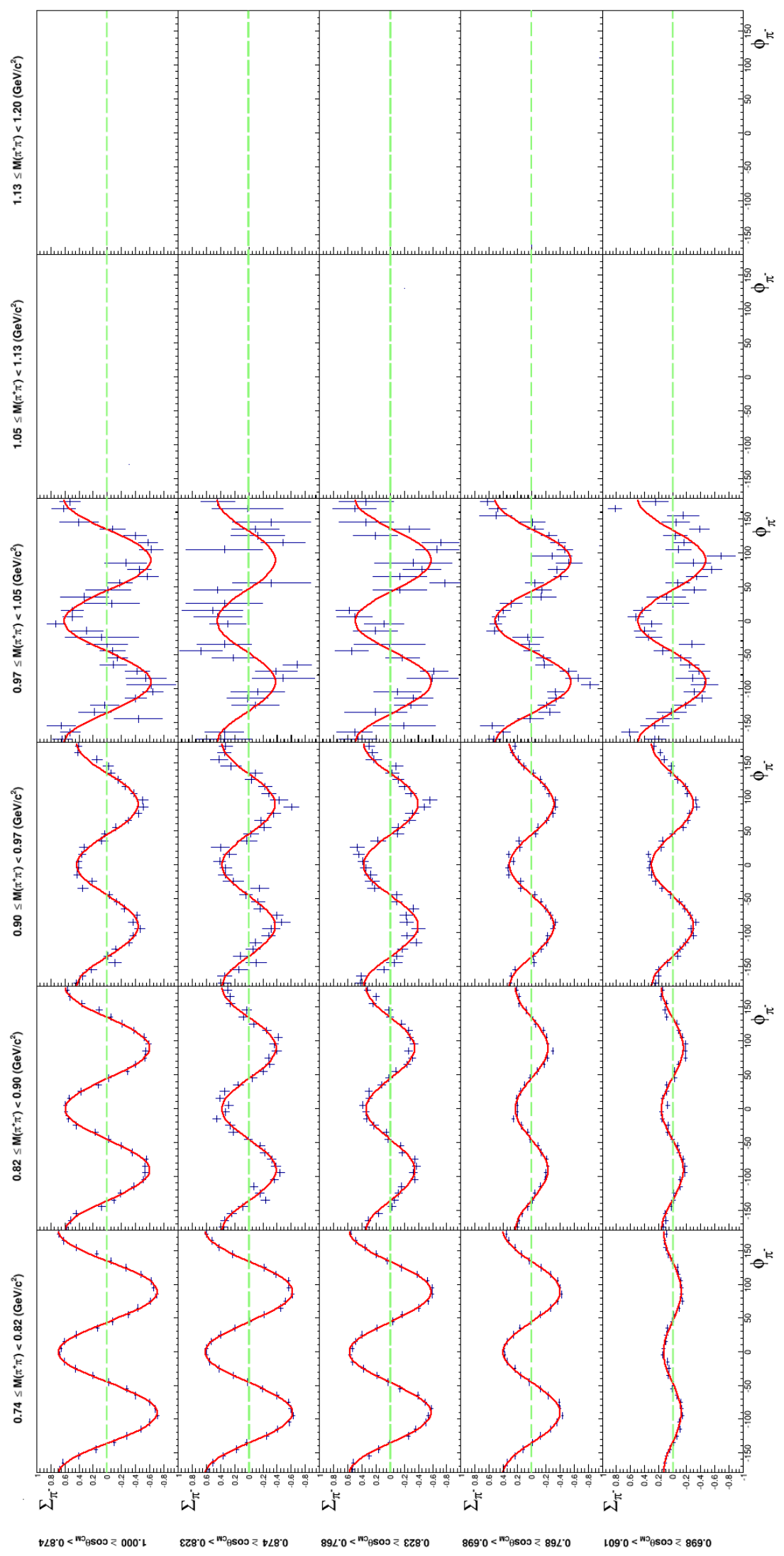

Figure B.6: The fit described in section 6.5 .1 for each kinematic region for extracting $\Sigma_{\pi^{-}}$for the second $\mathrm{W}$ region as defined in 6.2 .1 for the second half of the invariant mass distribution of the two pions in the forward angle region. 


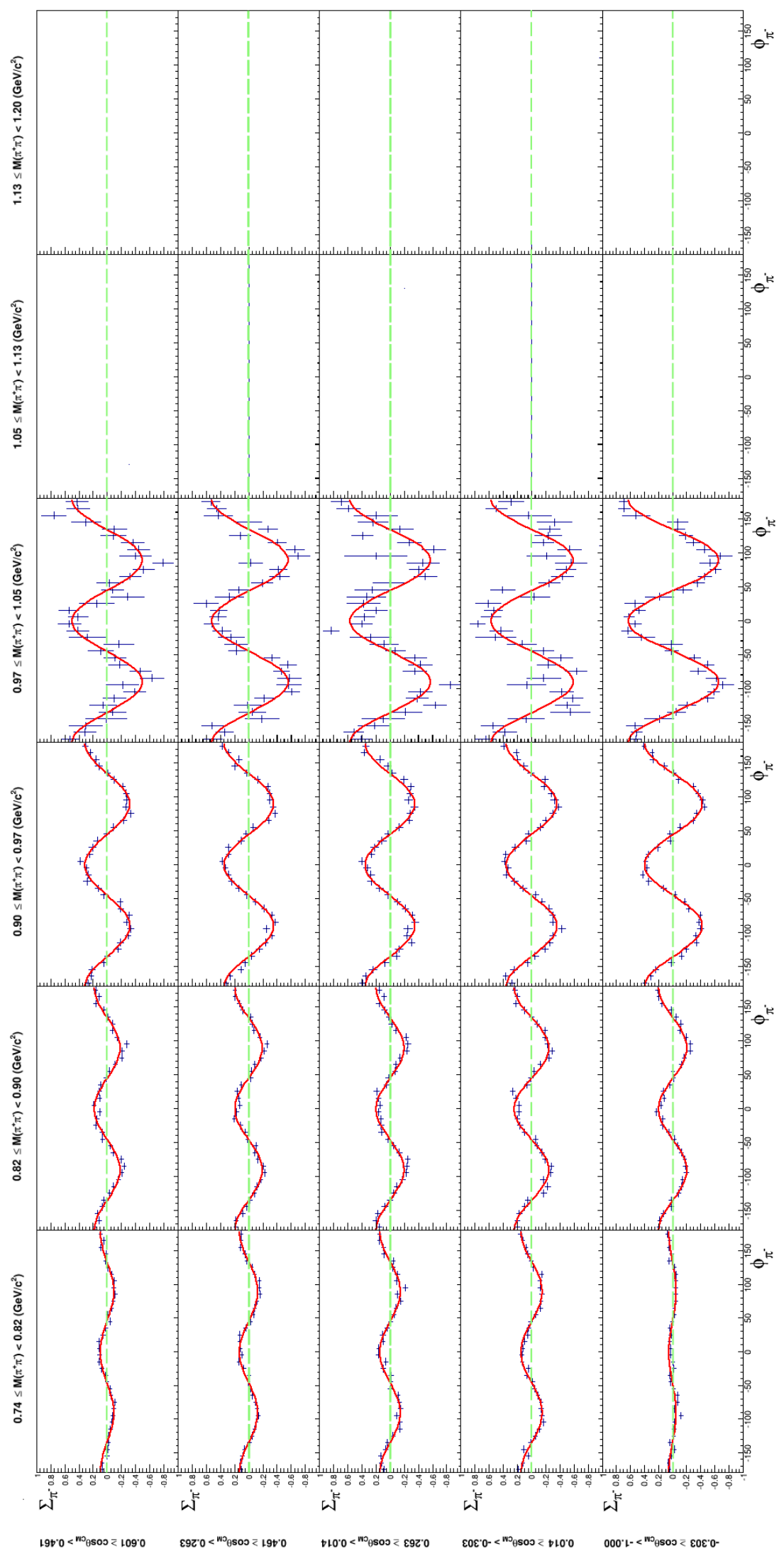

Figure B.7: The fit described in section 6.5 .1 for each kinematic region for extracting $\Sigma_{\pi^{-}}$for the second $\mathrm{W}$ region as defined in 6.2 .1 for the second half of the invariant mass distribution of the two pions in the backward angle region. 


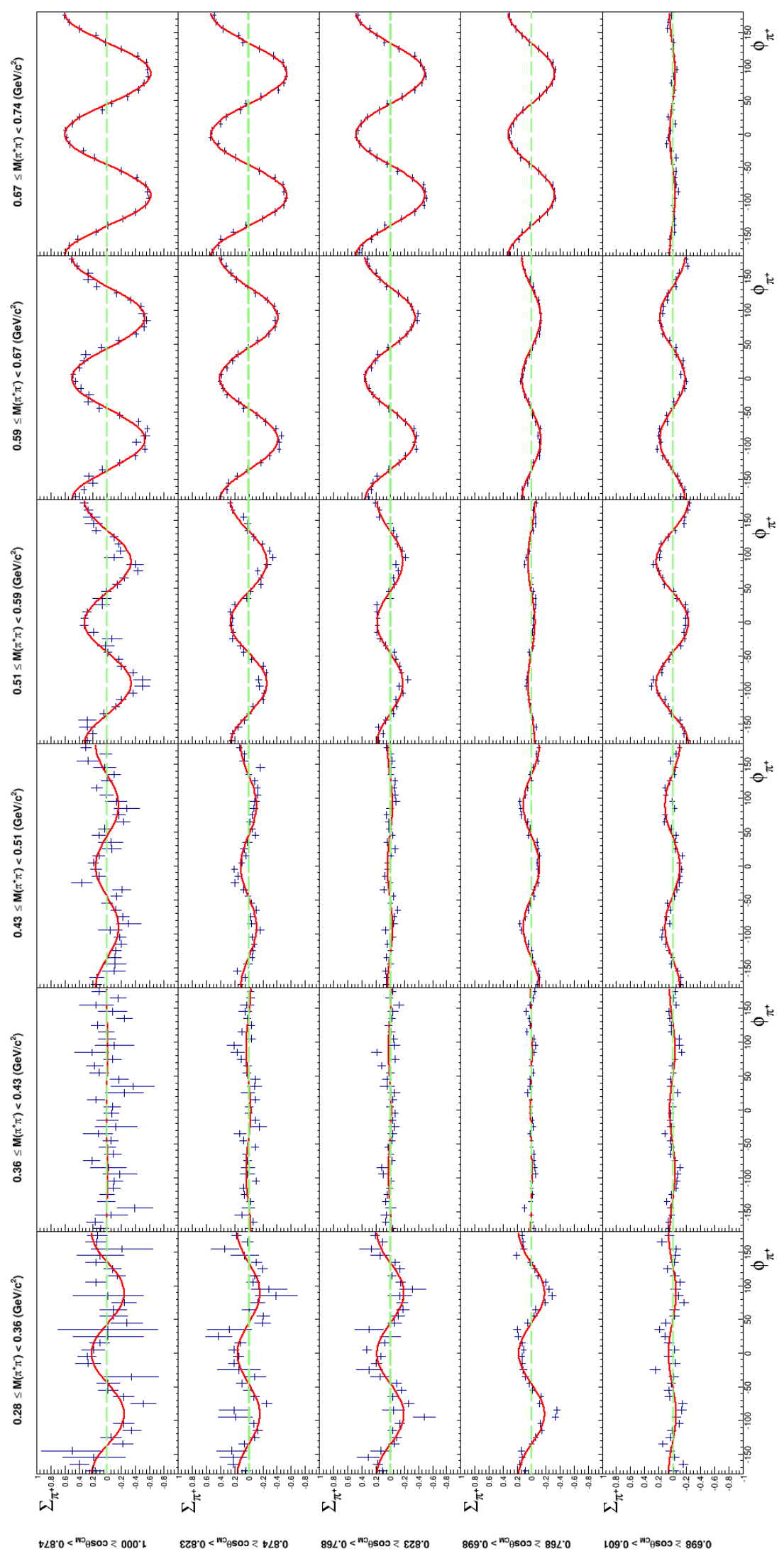

Figure B.8: The fit described in section 6.5 .1 for each kinematic region for extracting $\Sigma_{\pi^{+}}$for the second $\mathrm{W}$ region as defined in 6.2 .1 for the first half of the invariant mass distribution of the two pions in the forward angle region. 


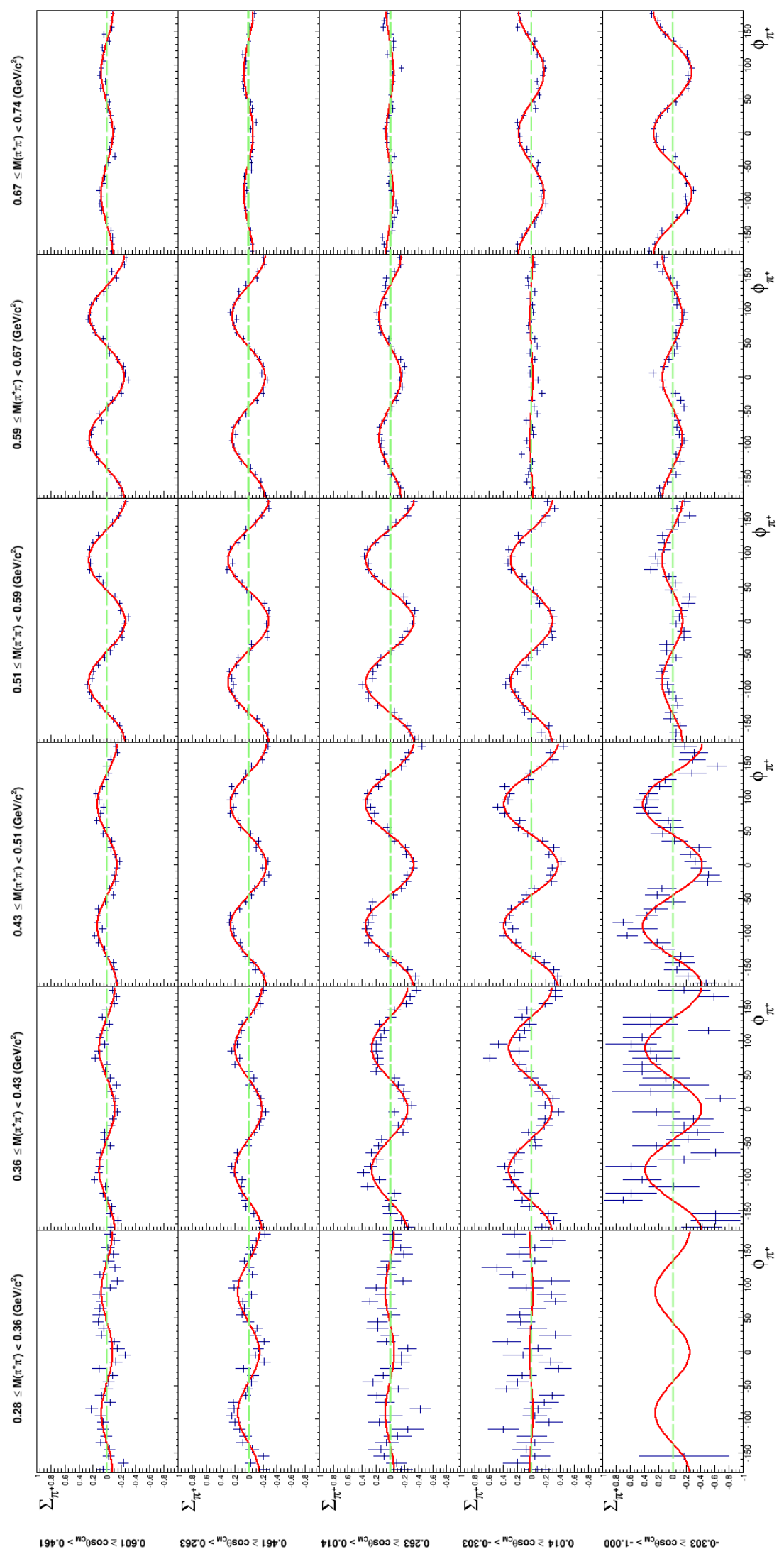

Figure B.9: The fit described in section 6.5 .1 for each kinematic region for extracting $\Sigma_{\pi^{+}}$for the second $\mathrm{W}$ region as defined in 6.2 .1 for the first half of the invariant mass distribution of the two pions in the backward angle region. 


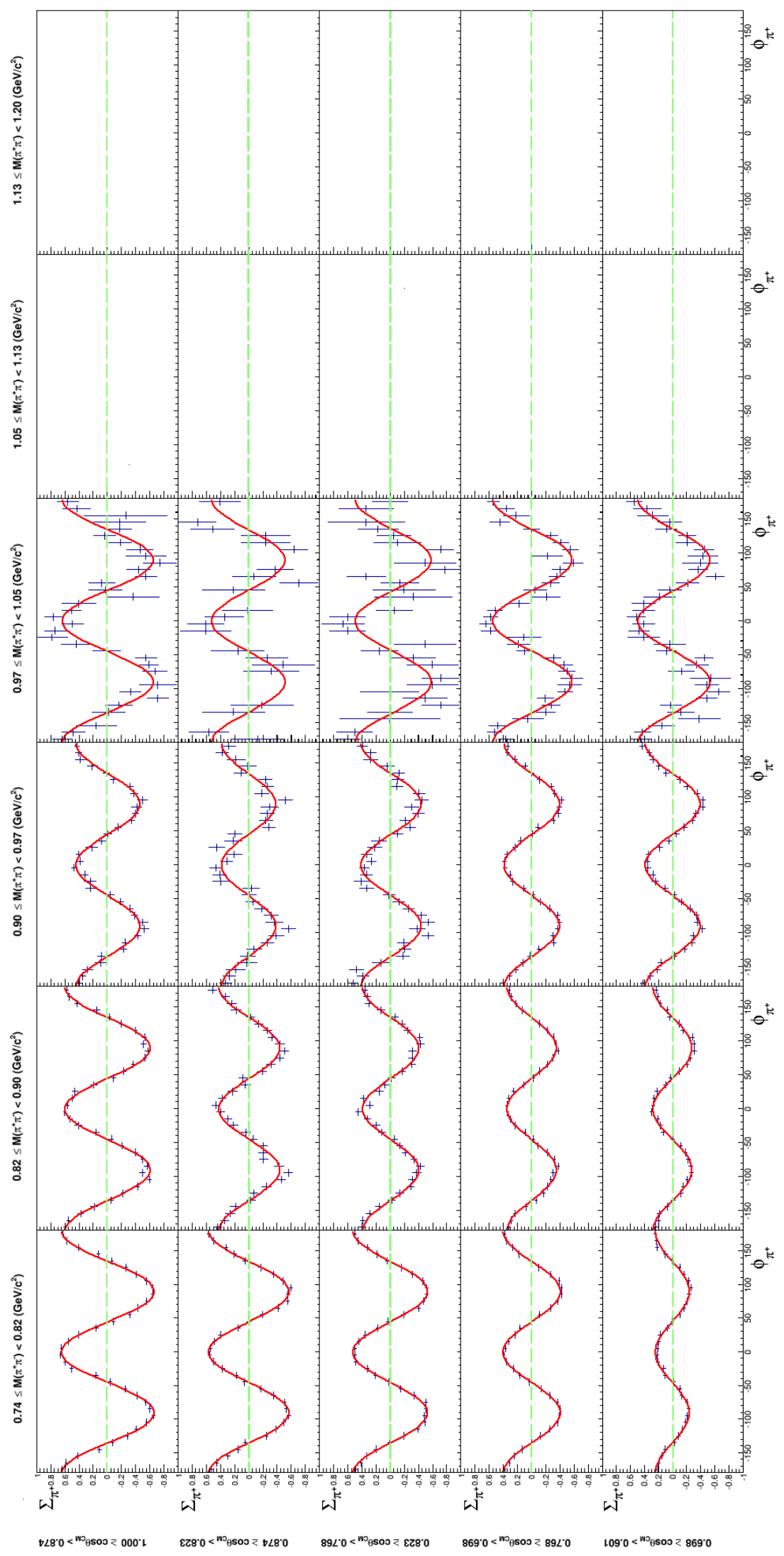

Figure B.10: The fit described in section 6.5 .1 for each kinematic region for extracting $\Sigma_{\pi^{+}}$for the second $\mathrm{W}$ region as defined in 6.2 .1 for the second half of the invariant mass distribution of the two pions in the forward angle region. 


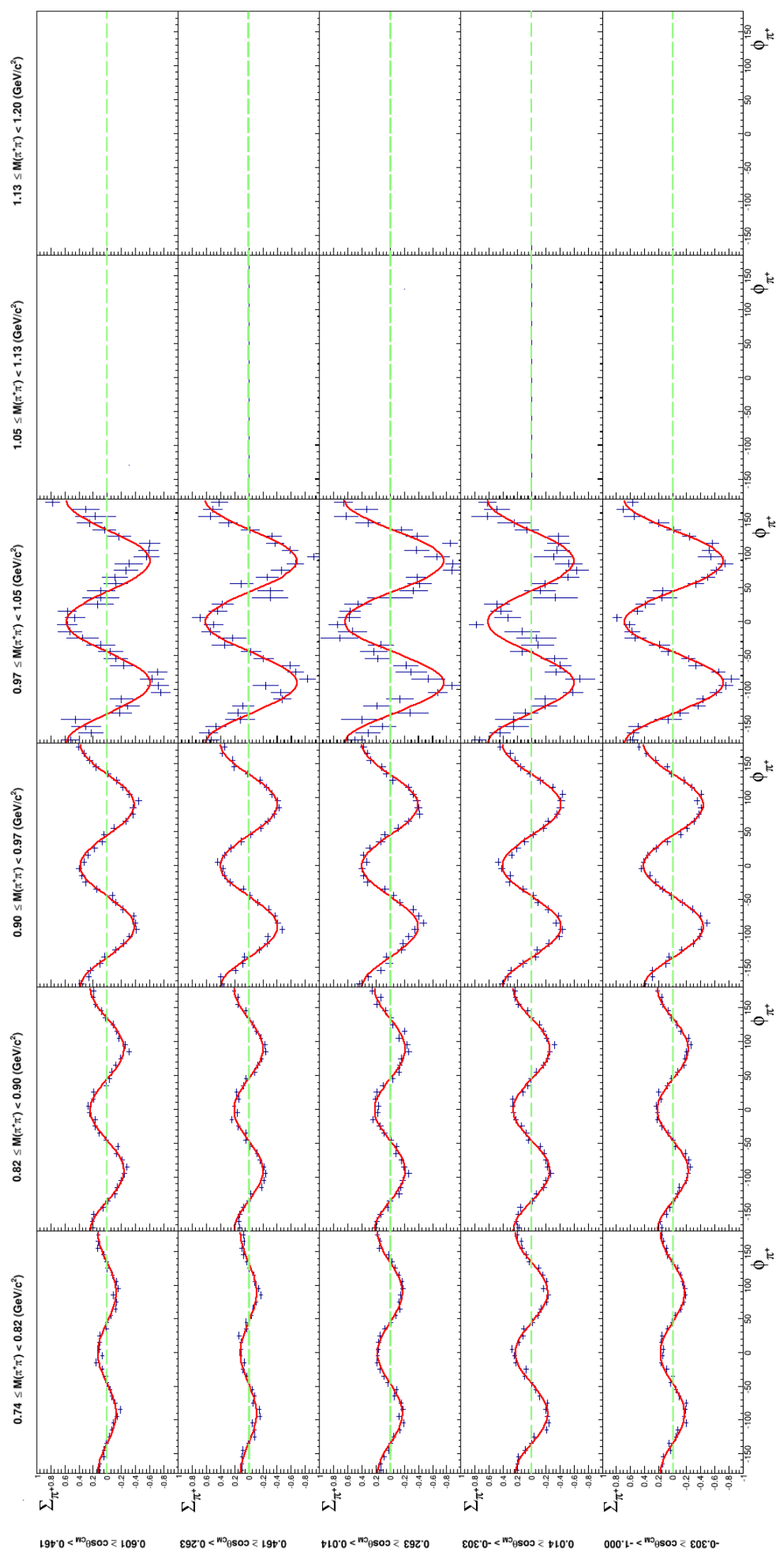

Figure B.11: The fit described in section 6.5 .1 for each kinematic region for extracting $\Sigma_{\pi^{+}}$for the second $\mathrm{W}$ region as defined in 6.2 .1 for the second half of the invariant mass distribution of the two pions in the backward angle region. 


\section{Bibliography}

[1] E. Rutherford. Collision of $\alpha$ particles with light atoms. Philosophy Magazine, 37:537, 1919.

[2] J. Chadwick. Possible existence of a neutron. Nature, 129:312, 1932.

[3] W. Heisenberg. Über den bau der atomkerne. Zeitschrift für Physik, 77:1, 1932.

[4] E. Wigner. On the consequences of the symmetry of the nuclear hamiltonian on the spectroscopy of nuclei. Physical Review, 51:106, 1937.

[5] E. C. G. Stueckelberg. Die wechselwirkungskräft in der elektrodynamik und in der feldtheorie der kernkräft. Helvetica Physica Acta, 11:299, 1938.

[6] M. Gell-Mann. Isotopic spin and new unstable particles. Physical Review, 92:833, 1953.

[7] K. Nishijima. Charge independence theory of v particles. Progress of Theoretical Physics, 13:285, 1955.

[8] M. Gell-Mann. The Eightfold Way: A theory of strong interaction symmetry. Synchrotron Laboratory Report CTSL-20, California Institute of Technology, 1993.

[9] Y. Ne'eman. Derivation of strong interactions from gauge invariance. Nuclear Physics, 26:222, 1961.

[10] http://www.cbooth.staff.shef.ac.uk/phy304/propquark.html. 
[11] M. Gell-Mann. A schematic model of baryons and mesons. Physics Letters, 8:214, 1964.

[12] G. Zweig. An SU(3) Model for Strong Interaction Symmetry and its Breaking. Report No.8182/TH.412, CERN, 1964.

[13] W. Pauli. Über den zusammenhang des abschlusses der elektronengruppen im atom mit der komplexstruktur der spektren. Zeitschrift für Physik, 31:765, 1925.

[14] B. Struminsky N. Bogoliubov and A. Tavkhelidze. On composite models in the theory of elementary particles. Joint Institute for Nuclear Research, D-1968, 1965.

[15] O. W. Greenberg. Spin and unitary-spin independence in a paraquark model of baryons and mesons. Physical Review Letters, 13:598, 1964.

[16] M. Y. Han and Y. Nambu. Three-triplet model with double su(3) symmetry. Physical Review B, 139:1006, 1965.

[17] E. D. Bloom et al. High-energy inelastic e - p scattering at $6^{\circ}$ and $10^{\circ}$. Physical Review Letters, 23:930, 1969.

[18] M. Breidenbach et al. Observed behavior of highly inelastic electron-proton scattering. Physical Review Letters, 23:935, 1969.

[19] J. Iliopoulos S. L. Glashow and L. Maiani. Weak interactions with lepton-hadron symmetry. Physical Review D, 2:1285, 1970.

[20] M. Kobayashi and T. Maskawa. Cp-violation in renormalizable theory of weak interaction. Progress of Theoretical Physics, 49:625, 1973.

[21] J.-E. Augustin et al. Discovery of a narrow resonance in $e^{+} e^{-}$annihilation. Physical Review Letters, 33:1406, 1974. 
[22] J. J. Aubert et al. Experimental observation of a heavy particle j. Physical Review Letters, 33:1404, 1974.

[23] S. W. Herb et al. Observation of a dimuon resonance at 9.5 gev in 400-gev proton-nucleus collisions. Physical Review Letters, 39:252, 1977.

[24] B. Carithers and P. Grannis. Discovery of the top quark. Beam Line (SLAC), 25:4, 1995 .

[25] D. Sokhan. Beam Asymmetry Measurements from Pion Photoproduction on the Neutron. PhD thesis, University of Edinburgh, 2009.

[26] H. D. Politzer. Reliable perturbative results for strong interactions? Physical Review Letters, 30:1346, 1973.

[27] S. Bethke. Experimental tests of asymptotic freedom. Progress in Particle and Nuclear Physics, 58:351, 2007.

[28] M. Williams. Measurement of Differential Cross Sections and Spin Density Matrix Elements along with a Partial Wave Analysis for $\gamma p \rightarrow p \omega$ using CLAS at Jefferson Lab. PhD thesis, Cernegie Mellon University, 2007.

[29] R. G. Edwards et al. Excited state baryon spectroscopy from lattice qcd. Physical Review D, 84:111502, 2011.

[30] V. Crede and W. Roberts. Progress toward understanding baryon resonances. Reports on Progress in Physics, 76:076301, 2013.

[31] S. Capstick and N. Isgur. Baryons in a relativized quark model with chromodynamic. Physical Review D, 34:2809, 1986.

[32] A. Vassallo J. Ferretti and e. Santopinto. Relativistic quark-diquark model of baryons. Physical Review C, 83:065204, 2011. 
[33] B. Ch. Metsch U. Löring and H. R. Petry. The light-baryon spectrum in a relativistic quark model with instanton-induced quark forces. European Physics Journal A, 10:447, 2001.

[34] B. Krusche. Photoproduction of Mesons off Nuclei - the Photonuclear Programs at ELS and MAMI. Journal of Physics, Conference Series:012003, 2012.

[35] N. Bianchi et al. Total hadron photoabsorption cross section on nuclei in the nucleon resonance region. Physical Review C, 54:1688, 1996.

[36] I. Zonta. Polarization observables from two pion and $\rho$ photoproduction on polarized HD target at JLab. PhD thesis, Università degli Studi di Roma, Tor Vergata, 2015.

[37] A. V. Anisovich and A. V. Sarantsev. Partial decay widths of baryons in the spinmomentum operator expansion method. European Physics Journal A, 30:427, 2006.

[38] G. Penner and U. Mosel. Vector meson production and nucleon resonance analysis in a coupled-channel approach for energies $m_{N}<\sqrt{s}<2$ gev. Physical Review C, 66:055212, 2002.

[39] http://www.kph.uni-mainz.de/maid/.

[40] R. L. Workman et al. Updated said analysis of pion photoproduction data. Physical Review C, 85:025201, 2012.

[41] V. D. Burkert. Status of the $n^{*}$ program at jefferson lab. European Physics Journal A, 17:303, 2002.

[42] I. Aznauryan et al. Results from $N^{*}$ Program at JLab. Journal of Physics: Conference Series, 299, 2011.

[43] P.J. Cole et al. Photoproduction of the $\rho$ Meson from the Proton with Linearly Polarized Photons. CEBAF Program Advisory Comittee Nine, 1994. 
[44] R. Koniuk and N. Isgur. Baryon decays in a quark model with chromodynamics. Physical Review D, 21:1868, 1980.

[45] S. Capstick and N. Isgur. N $\pi$ decays of baryons in a relativized model. Physical Review D, 47:1994, 1993.

[46] D. M. Manley and E. M. Saleski. Multichannel resonance parametrization of

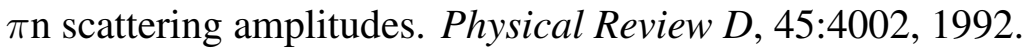

[47] Y. Eisenberg et al. Rho meson photoproduction cross-sections in the strong absorption model. Physics Letters, 22:217-219, 1966.

[48] R. L. Anderson et al. A determination of the rho-nucleon cross section from elastic rho-photoproduction on deuterium. Physical Review D, 4:3245, 1971.

[49] M. Battaglieri et al. Measurement of Direct $f_{0}(980)$ Photoproduction on the Proton. Physical Review Letters, 102:102001, 2009.

[50] M. Battaglieri et al. Photoproduction of $\pi^{+} \pi^{-}$meson pairs on the proton. Physical Review D, 80, 2009.

[51] K.A. Olive et al (Particle Data Group). Chinese Physics C, 38:090001, 20014.

[52] J-H Yoon. On the mass difference between $\pi$ and $\rho$ using a relativistic two-body model. Few-Body Systems, 54:475-478, 2013.

[53] J. R. Peláez. Nature of light scalar mesons from their large- $\mathrm{N}_{c}$ behaviour. Physical Review Letters, 92:10, 2004.

[54] L. Maiani et al. New look at scalar meson. Physical Review Letters, 93:212002, 2004.

[55] G. Adera. Relativistic Description of Two-body Scattering Reactions. $\mathrm{PhD}$ thesis, African Institute for Mathematical Science, 2007. 
[56] G. Kramer and K. Schilling. Influence of absorption on peripheral photoproduction of vector mesons. Zeitschrift für Physik, 191:51-69, 1966.

[57] S. Mandelstam. Determination of the pion-nucleon scattering amplitude from dispersion relations and unitarity general theory. Physical Review, 112:1344, 1958.

[58] P. Landshoff S. Donnachie, G. Dosch and O. Nachtmann. Pomeron Physics and QCD. Cambridge University Press, 2002.

[59] C.I.O. Gordon. $\rho^{0}$ Photoproduction Using Linearly Polarised Photons with the CLAS Detector. PhD thesis, University of Glasgow, 2004.

[60] J.-M. Laget. Photoproduction of vector mesons at large transfer. Physics Letters $B, 489: 313-318,2000$.

[61] M. D. Schwartz. Quantum Field Theory and the Standard Model. Cambridge University Press, 2014.

[62] M. Pichowsky and Ç. Şavlki and F. Tabakin. Polarization observables in vector meson photoproduction. Physical Review C, 53(2):593-610, 1996.

[63] M. Jacob and G.C. Wick. On the General Theory of Collisions for Particles with Spin. Annals of Physics, 7:404-428, 1959.

[64] A.I. Titov et al. Photoproduction of $\phi$ mesons from the proton: Polarization observables and strangeness in the nucleon. Physical Review C, 58:2429-2449, 1998.

[65] K. Schilling. On the analysis of vector-meson production by polarized photons. Nuclear Physics, B15:397-412, 1970.

[66] A. Pautz. Vector Meson Photoproduction. PhD thesis, University of Manchester, 1995. 
[67] C. W. Salgado and D. P. Weygand. On the partial-wave analysis of mesonic resonances decaying to multiparticle final states produced by polarized photons. Physics Reports, 537:1-58, 2014.

[68] K. Gottfried and J. D. Jackson. On the connection between production mechanism and decay of resonances at high energies. Il Nuove Cimento Series 10, 33:309-330, 1964.

[69] E. Leader. Spin in Particle Physics. Cambridge University Press, 2001.

[70] J.A.S. Bernal. The Photoproduction of $\phi$-mesons off Protons by Using a Beam of Linearly Polorized Photons at Threshold Energies. PhD thesis, Idaho State University, 2009.

[71] W.-T. Chaing W. M. Kloet and F. Tabakin. Spin information from vector-meson decay in photoproduction. Physical Review C, 58:1086, 1998.

[72] A. I. Titov Y. Oh and T.-S. H. Lee. Nucleon resonances in polarized $\omega$ photoproduction. AIP Conference Proceedings, 570:546, 2001.

[73] L. Criegee et al. Rho production with polarized photons. Physics Letters, 28B:282-286, 1968.

[74] J.-M. Laget and R. Mendez-Galain. Exclusive photo- and electroproduction of vector mesons at large momentum transfer. Nuclear Physics A, 581:397-428, 1994.

[75] J. Ballam et al. Bubble-chamber study of photoproduction by 2.8- and 4.7-gev polarized photons. Physical Review D, 5:545, 1972.

[76] J. Ballam et al. Vector-meson production by polarized photons at 2.8, 4.7, and 9.3 gev. Physical Review D, 7:3150, 1973.

[77] L. Criegee et al. Rho production with linearly polarized photons. Physical Review Letters, 25:1306, 1970. 
[78] G. Diambrini-Palazzi et al. Photoproduction of rho mesons from hydrogen and carbon by linearly polarized photons. Physical Review Letters, 25:478, 1970.

[79] ABBHHM Collaboration. Photoproduction of meson and baryon resonances at energies up to $5.8 \mathrm{GeV}$. Physical Review, 175:1669, 1968.

[80] J. Ajaka et al. Evidence for nucleon-resonance excitation in $\omega$-meson photoproduction. Physical Review Letters, 96:132003, 2006.

[81] A. I. Titov et al. Effective lagrangian approach to the $\omega$ photoproduction near threshold. Physical Review C, 66:15204, 2002.

[82] V. Shklyar et al. Coupled-channel analysis of $\omega$-meson production in $\pi n$ and $\gamma n$ reactions for c.m. energies up to 2 gev. Physical Review C, 71:055206, 2005.

[83] F. J. Klein. Vector meson photoproduction at the SAPHIR detector. PhD thesis, Bonn University, 2009.

[84] Q. Zhao et al. Vector meson photoproduction with an effective lagrangian in the quark model. Physical Review C, 58:2393-2413, 1998.

[85] C. Hanretty. Measurement of Polarization Observables $I^{s}$ and $I^{C}$ for $\bar{\gamma} p \rightarrow$ $p \pi^{+} \pi^{-}$Using the CLAS Spectrometer. PhD thesis, The Florida State University, 2013.

[86] D. R. Douglas C. W. Leeman and G. A. Krafft. The Continuous Electron Beam Accelerator Facility: CEBAF at the Jefferson Laboratory. Annual Review of Nuclear and Particle Science, 51:413-450, 2001.

[87] B. A. Mecking et al. The CEBAF Large Acceptance Spectrometer. Nuclear Instruments and Methods A, 503:513, 2003.

[88] S. Stepanyan et al. The CEBAF Large Acceptance Spectrometer. Nuclear Instruments and Methods A, 572:654, 2007. 
[89] D. I. Sober et al. The Bremmstrahlung Tagged Photon Beam in Hall B at JLab. Nuclear Instruments and Methods A, 440:263, 2000.

[90] J. Kellie et al. The selection and performance of diamond radiators used in coherent bremsstrahlung experiments. Nuclear Instruments and Methods A, 545:164-180, 2005.

[91] K. Livingston. The Stonehenge technique. A method for aligning coherent bremmsstrahlung radiators. Nuclear Instruments and Methods A, 603:205-213, 2009.

[92] P. Cole. The ipn-orsay/utep intrumented collimator. JLab Internal, 1999.

[93] J. O’Meara et al. A Superconducting Toroidal Magnet for the CEBAF Large Acceptance Spectrometer. IEEE Transactions on Magnetics, 25:1902, 1989.

[94] S. Taylor et al. The CLAS Start Counter. Nuclear Instruments and Methods A, 462:484, 2001.

[95] Y. G. Sharabian et al. A new highly segmented start counter for the CLAS detector. Nuclear Instruments and Methods A, 556:246, 2006.

[96] F. J. Barbosa et al. A drift chamber system for a toroidal detector. Nuclear Instruments and Methods A, 323:191, 1992.

[97] M. D. Mestayer et al. The CLAS Drift Chamber System. Nuclear Instruments and Methods A, 449:81, 2000.

[98] G. Adams et al. The CLAS Cerenkov Detector. Nuclear Instruments and Methods A, 465:414, 2001

[99] M. Amarian et al. The CLAS Forward Electromagnetic Calorimeter. Nuclear Instruments and Methods A, 460:484, 2001. 
[100] E. S. Smith et al. The Time-of-Flight System for CLAS. Nuclear Instruments and Methods A, 432:265, 1999.

[101] D. C. Doughty et al. A VXIbus based trigger for the CLAS detector at CEBAF. Nuclear Science IEEE Transactions, 39:241-247, 1992.

[102] D. Cords et al. CLAS Event Format with BOS. CLAS Note, 94-012, 1994.

[103] J. J. Sakurai. Modern Quantum Mechanics. The Benjamin Cummings Publishing Company, Inc., 1985.

[104] K. S. Krane. Introductory Nuclear Physics. John Wiley \& Sons, 1987.

[105] U. Timm. Coherent Bremsstrahlung of Electrons in Crystals. Fortschritte der Physik, 17:765-808, 1969.

[106] H. Überall. High-energy interference effect of bremmstrahlung and pair production in crystals. Physical Review, 103:1055-1067, 1956.

[107] H. Überall. Polarization of bremsstrahlung from monocrystalline targets. Physical Review, 107:223-227, 1956.

[108] M. M. May. On the polarization of high energy bremsstrahlung and of high energy pairs. Physical Review, 84:265-270, 1951.

[109] K. Livingston. Polarization from Coherent Bremsstrahlung Enhancement. CLAS NOTE 2011-020, 2011.

[110] N. Zachariou. Determination of the Azimuthal Asymmetry of Deuteron Photodisintegration in the Energy Region $E_{\gamma}=1.1-2.3 \mathrm{GeV}$. PhD thesis, The Columbian College of Arts and Sciences of The George Washington University, 2011.

[111] M. Dugger and B. Ritchie. Consistency corrections to the linear photon polarization for g8b data. CLAS Note 2012-02, 2012. 
[112] M. Dugger et al. Extraction technique for sigma and $\mathrm{g}$ for pseudoscalar meson photoproduction. CLAS Note (In Progress), 2015.

[113] F. Klein et al. Beam asymmetries in near-threshold $\omega$ photoproduction off the proton. Physical Review D, 78, 2008.

[114] D. Ireland et al. Strangeness Photoproduction Polarization Observables from g8. CLAS Note(in progress), 2015.

[115] M. Dugger and C. Henretty. Correction to the incident photon energy for g8b data. CLAS Note 2009-030, 2009.

[116] E. Pasyuk. Energy loss corrections for charged particles in CLAS. CLAS Note 2007-016, 2007.

[117] I. Jaegle et al. Quasi-free photoproduction of $\eta$-mesons off the neutron. Physical Review Letters, 100:252002, 2008.

[118] V. Kuznetsov et al. Evidence for a narrow structure at $\mathrm{W} \sim 1.68 \mathrm{MeV}$ in $\eta$ photoproduction off the neutron. Physics Letters B, 647:23-29, 2007.

[119] B. Vernarsky. First Measurements of the Polarized Spin Density Matrix Elements along with a Partial-Wave Analysis for $\gamma p$ to $p w$ using CLAS at Jefferson Lab. PhD thesis, Carnegie Mellon University, 2014.

[120] M. Guidal. Exclusive $\rho^{0}$ electroproduction on the proton at clas. European Physical Journal A, 39:5-31, 2008.

[121] A. Fradi. Electroproduction exlusive du méson $\rho^{+}$sur le proton à JLab avec le détectuer CLAS. PhD thesis, Université Paris-Sud XI, 2009.

[122] M. Ross and L. Stodolsky. Photon Dissociation Model for Vector-Meson Photoproduction. Physical Review, 149:1172-1181, 1966. 
[123] P. Söding. On the apparent shift of the rho meson mass in photoproduction. Physics Letters, 19:702-704, 1966.

[124] M. Battaglieri et al. Analysis tools for next-generation hadron spectroscopy experiments. Acta Physica Polonica B, 46, 2015. 